\title{
Synthesis of Chiral a-Amino Tertiary Boronates via the Catalytic Enantioselective Nucleophilic Borylation of Dialkyl Ketimines
}

Koji Kubota, ${ }^{* \dagger, \ddagger}$ Daiyo Miura, ${ }^{\dagger}$ Takumi Takeuchi, ${ }^{\dagger}$ Shun Osaki ${ }^{\dagger}$ and Hajime Ito* ${ }^{* \dagger} \ddagger$

†Division of Applied Chemistry, Graduate School of Engineering, Hokkaido University, Sapporo, Hokkaido 060-8628, Japan, FAX: +81-(0)11-706-6561

Institute for Chemical Reaction Design and Discovery (WPI-ICRD), Hokkaido University, Sapporo, Hokkaido 001-0021, Japan

Corresponding Author

kbt@eng.hokudai.ac.jp, hajito@eng.hokudai.ac.jp

\section{Table of Contents}

1. General and Materials

2. Substrate Synthesis

3. General Experimental Procedures for Borylation of Ketimines

4. Experimental Procedures for the Synthesis of Peptydilboronic Acid Derivatives

5. Additional Experimental Results

6. Determination of Absolute Configuration of Borylation Product

7. Plausible Catalytic Cycle

8. DFT Calculations

9. Analysis for Non-Covalent Interactions in the Transition States

10. Reference

11. HPLC Charts

12. ${ }^{1} \mathrm{H}$ and ${ }^{13} \mathrm{C}$ NMR Spectra 


\section{General and Materials}

All reactions were performed in oven-dried glassware using conventional Schlenk techniques under a static pressure of nitrogen or argon. Materials were obtained from commercial suppliers and used as received unless otherwise noted. Dry solvents for the reactions were purchased from commercial suppliers, degassed via three freeze-pump-thaw cycles, and further dried over molecular sieves (MS4A) prior to use. 1,3-Dicyclohexylimidazolium chloride ( $\mathrm{ICy} \cdot \mathrm{HCl},>98.0 \%)$ and $\mathrm{K}(\mathrm{O}-t-\mathrm{Bu})$ $(>97.0 \%)$ purchased from Tokyo Chemical Industry Co. The chiral $N$-heterocyclic carbene (NHC)

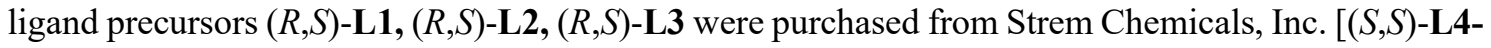
$(S, S)-\mathbf{L} 7^{1},(S, S)-\mathbf{L} \mathbf{8}^{2},(S, S)$-L. $\mathbf{9}^{2}$ and $\left.(R, R)-\mathbf{L} \mathbf{1 0}^{3}\right]$ were synthesized according to the literature. $(R)$ DTBM-SEGPHOS $[(R)$-L11] was purchased from Tokyo Chemical Industry Co. $(R, R)$-Quinox P* $[(R, R)-\mathbf{L 1 2}]$ was obtained from Nippon Chemical Industrial Co. Silica Gel $60 \mathrm{~N}(40-100 \mu \mathrm{m}$, spherical, neutral) purchased from Kanto Chemical Co. was used as received. NMR spectra were recorded on JEOL JNM-ECX400P, ECS-400 ( ${ }^{1} \mathrm{H}: 392$ or $396 \mathrm{MHz},{ }^{13} \mathrm{C}$ : 99 or $\left.100 \mathrm{MHz}\right)$, and JNM-ECA600 $\left({ }^{13} \mathrm{C}\right.$ : $151 \mathrm{MHz}$ ). Tetramethylsilane ( $\delta=0.00 \mathrm{ppm}$ for $\left.{ }^{1} \mathrm{H} \mathrm{NMR}\right)$ and $\mathrm{CDCl}_{3}\left(\delta=77.0 \mathrm{ppm}\right.$ for $\left.{ }^{13} \mathrm{C} \mathrm{NMR}\right)$ were employed as external standards. $\mathrm{BF}_{3} \cdot \mathrm{Et}_{2} \mathrm{O}$ was used as an external standard for ${ }^{11} \mathrm{~B}$ NMR analysis. $\mathrm{D}_{3} \mathrm{PO}_{4}$ in $\mathrm{D}_{2} \mathrm{O}$ was used as an external standard for ${ }^{31} \mathrm{P}$ NMR analysis. Multiplicity was reported as follows: $\mathrm{s}=$ singlet, $\mathrm{brs}=$ broad singlet, $\mathrm{d}=$ doublet, $\mathrm{t}=$ triplet, $\mathrm{q}=$ quartet, quint $=$ quintet, $\mathrm{sept}=$ septet, $\mathrm{m}=$ multiplet. 1,1,2,2-Tetrachloroethane was used as an internal standard for determining NMR yield. NMR yield was determined by quantitative ${ }^{1} \mathrm{H}-\mathrm{NMR}$ analysis of the crude reaction mixture. GLC analyses were conducted with a Shimadzu GC-2014 or GC-2025 equipped with ULBON HR-1 glass capillary column (Shinwa Chemical Industries) and an FID detector. Recycle preparative gel chromatography (GPC) was conducted with JAILC-9101 using $\mathrm{CHCl}_{3}$ as an eluent. FTIR spectra were recorded on a JASCO FT IR 4700 spectrometer. Single crystal X-ray structural analyses were carried out on an XtaLAB PRO MM007 diffractometer using graphite monochromated $\mathrm{Cu}-\mathrm{K}_{\alpha}$ radiation. The structure was solved by direct methods and expanded using Fourier techniques. Non-hydrogen atoms were refined anisotropically. Hydrogen atoms were refined using the riding model. All calculations were performed using the Olex 2 crystallographic software package except for refinement, which was performed using SHELXL-2013. High-resolution mass spectra were recorded at the Global Facility Center for Instrumental Analysis, Hokkaido University. 


\section{Substrate Synthesis}

\section{List of the substrate used in this study.}<smiles>C/C(=N\P(=O)(c1ccccc1)c1ccccc1)C1CCCCC1</smiles>

$1 \mathrm{a}$<smiles>CCCC(C)(C)C(C)=NP(=O)(c1ccccc1)c1ccccc1</smiles>

$1 f$<smiles>CCCC/C(=N\P(=O)(c1ccccc1)c1ccccc1)C(C)(C)C</smiles>

$1 k$<smiles>CC(=NP(=O)(CO)OCCO)C1CCCCC1</smiles>

$1 b$<smiles>C/C(=N\P(=O)(c1ccccc1)c1ccccc1)C1(c2ccccc2)CCC1</smiles>

$1 \mathrm{~g}$<smiles>C/C(CC(C)C)=N\P(=O)(c1ccccc1)c1ccccc1</smiles>

11<smiles>C/C(=N\P(=O)([O-])[O-])C1CCCCC1</smiles>

$1 c$<smiles>C/C(=N\P(=O)(c1ccccc1)c1ccccc1)C1(c2ccccc2)CCCCC1</smiles>

$1 \mathrm{~h}$<smiles>C/C(CCc1ccccc1)=N\P(=O)(c1ccccc1)c1ccccc1</smiles>

$1 \mathrm{~m}$<smiles>CC(C)C(=NP(=O)([O-])O[Na])O[Na]</smiles>

$1 d$<smiles>C/C(=N\P(=O)(c1ccccc1)c1ccccc1)C12CC3CC(C1)C(C3)C2</smiles>

$1 \mathrm{i}$<smiles>CC/C(C)=N/P(=O)(c1ccccc1)c1ccccc1</smiles>

$1 \mathrm{n}$<smiles>CC(=NP(=O)([O-])OCO)C(C)(C)C</smiles>

$1 e$<smiles>C/C(=N\P(=O)(c1ccccc1)c1ccccc1)C1CC1</smiles>

1j

The spectroscopic data of $1 \mathbf{a}$ and 1e were consistent with literature values. ${ }^{4,5}$ The $E / Z$ configurations of other substrates were deduced relative to these compounds.

\section{Procedure A.}

(E)-N-(1-Cyclohexylethylidene)-P,P-diphenylphosphinic amide (1a).

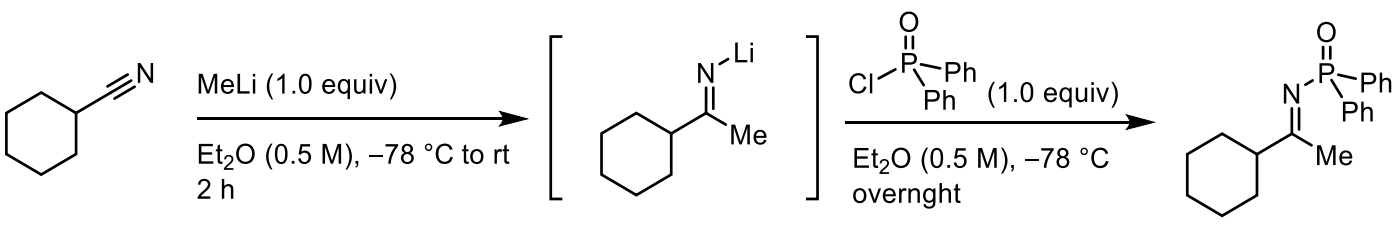

$1 a$

$\operatorname{MeLi}\left(\mathrm{Et}_{2} \mathrm{O} 1.1 \mathrm{M}\right)(9.1 \mathrm{~mL}, 10 \mathrm{mmol}, 1.0$ equiv) was added dropwise to a suspension of cyclohexanecarbonitrile (1.2 mL, $10 \mathrm{mmol}, 1.0$ equiv) in $\mathrm{Et}_{2} \mathrm{O}(20 \mathrm{~mL})$ at $-78{ }^{\circ} \mathrm{C}$ under nitrogen atmosphere. The reaction mixture was allowed to warm to room temperature and stirred for $2 \mathrm{~h}$. Then, the reaction mixture was cooled down to $-78{ }^{\circ} \mathrm{C}$, and diphenyl phosphinic chloride $(1.91 \mathrm{~mL}, 10 \mathrm{mmol}$, 1.0 equiv) was added dropwise to the mixture. The reaction mixture was kept at $-78^{\circ} \mathrm{C}$ and stirred 
overnight. The mixture was directly filtered through a short silica-gel column with EtOAc as an eluent, and then the resultant solution was concentrated under reduced pressure. The crude product was purified by silica-gel column chromatography with EtOAc/Hex eluent (100:0 to 30:70). Further purification was conducted by GPC to afford the corresponding ketimine 1a $(280.5 \mathrm{mg}, 0.86 \mathrm{mmol}$, $9 \%$ yield) as a white solid. The spectroscopic data were matched in those reported. ${ }^{4}$

${ }^{1} \mathrm{H}$ NMR (392 MHz, $\left.\mathrm{CDCl}_{3}, \delta\right): 1.17-1.46$ (m, 5H), 1.71-1.95 (m, 5H), 2.35-2.43 (m, 1H), 2.47 (d, $J=1.6 \mathrm{~Hz}, 3 \mathrm{H}), 7.39-7.47(\mathrm{~m}, 6 \mathrm{H}), 7.89-7.94(\mathrm{~m}, 4 \mathrm{H})$. HRMS-EI $(\mathrm{m} / \mathrm{z})$ : $[\mathrm{M}]^{+}$calcd for $\mathrm{C}_{20} \mathrm{H}_{24} \mathrm{NOP}$, 325.1596; found, 325.1598 .

\section{(E)- $N$-(1-Cyclohexylethylidene)-P,P-di-m-tolylphosphinic amide (1b).}

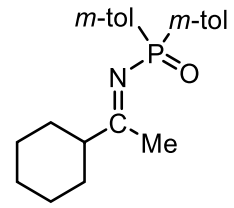

1b

1b was prepared from the corresponding nitrile according to procedure A. The product $\mathbf{1 b}$ was obtained in $26 \%$ yield ( $373.7 \mathrm{mg}, 1.1 \mathrm{mmol}$, yellow oil) from the corresponding nitrile (4.0 mmol).

${ }^{1} \mathrm{H}$ NMR $\left(392 \mathrm{MHz}, \mathrm{CDCl}_{3}, \delta\right.$ ): 1.19-1.49 (m, 5H), 1.70-1.94 (m, 5H), 2.36 (s, 6H), 2.38-2.42 (m, 1H), $2.45(\mathrm{~d}, J=2.4 \mathrm{~Hz}, 3 \mathrm{H}), 7.24-1.34(\mathrm{~m}, 4 \mathrm{H}), 767-7.76(\mathrm{~m}, 4 \mathrm{H}) .{ }^{13} \mathrm{C} \mathrm{NMR}\left(100 \mathrm{MHz}, \mathrm{CDCl}_{3}, \delta\right)$ : $21.5\left(\mathrm{CH}_{3}\right), 25.2\left(\mathrm{~d}, J=13.4 \mathrm{~Hz}, \mathrm{CH}_{3}\right), 25.99\left(\mathrm{CH}_{2}\right), 26.04\left(\mathrm{CH}_{2}\right), 30.2\left(\mathrm{CH}_{2}\right), 52.1(\mathrm{~d}, J=20.1 \mathrm{~Hz}$, $C \mathrm{H}), 128.2(\mathrm{~d}, J=13.4 \mathrm{~Hz}, C \mathrm{H}), 128.7$ (d, $J=8.6 \mathrm{~Hz}, C \mathrm{H}), 132.0(\mathrm{~d}, J=5.8 \mathrm{~Hz}, C \mathrm{H}), 132.1(C \mathrm{H})$, 134.9 (d, $J=130.3 \mathrm{~Hz}, C), 138.1(\mathrm{~d}, J=13.4 \mathrm{~Hz}, C), 195.4(\mathrm{~d}, J=11.5 \mathrm{~Hz}, C) .{ }^{31} \mathrm{P}\left\{{ }^{1} \mathrm{H}\right\} \mathrm{NMR}(159$ $\mathrm{MHz}, \mathrm{CDCl}_{3}, \delta$ ): 18.4. HRMS-EI $(\mathrm{m} / \mathrm{z})$ : $[\mathrm{M}]^{+}$calcd for $\mathrm{C}_{22} \mathrm{H}_{28} \mathrm{NOP}, 353.1909$; found, 353.1908. IR (neat, $\left.\mathrm{cm}^{-1}\right): 1187(\mathrm{P}=\mathrm{O}), 1652(\mathrm{C}=\mathrm{N})$.

\section{(E)-N-(1-Cyclohexylethylidene)-P,P-di-o-tolylphosphinic amide (1c).}

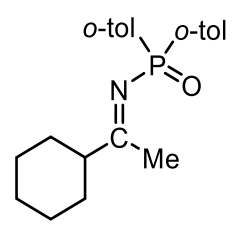

$1 c$

1c was prepared from the corresponding nitrile according to procedure A. The product 1c was obtained in $4 \%$ yield $(187.4 \mathrm{mg}, 0.50 \mathrm{mmol}$, white solid) from the corresponding nitrile (13.6 mmol).

${ }^{1} \mathrm{H}$ NMR $\left(392 \mathrm{MHz}, \mathrm{CDCl}_{3}, \delta\right): 1.22-1.39$ (m, 5H), 1.70-1.94 (m, 5H), 2.30 (s, 6H), 2.34-2.39 (m, 1H), $2.41(\mathrm{~d}, J=1.8 \mathrm{~Hz}, 3 \mathrm{H}), 7.14-7.17(\mathrm{~m}, 2 \mathrm{H}), 7.28-7.30(\mathrm{~m}, 2 \mathrm{H}), 7.35-7.41(\mathrm{~m}, 2 \mathrm{H}), 8.08$ (ddd, $J$ 
$=1.0,7.8,15.7 \mathrm{~Hz}, 2 \mathrm{H}) \cdot{ }^{13} \mathrm{C} \mathrm{NMR}\left(99 \mathrm{MHz}, \mathrm{CDCl}_{3}, \delta\right): 21.7\left(\mathrm{~d}, J=2.9 \mathrm{~Hz}, \mathrm{CH}_{3}\right), 25.3(\mathrm{~d}, J=14.2$ $\left.\mathrm{Hz}, \mathrm{CH}_{3}\right), 26.1\left(\mathrm{CH}_{2}\right), 30.3\left(\mathrm{CH}_{2}\right), 52.6(\mathrm{~d}, J=20.8 \mathrm{~Hz}, \mathrm{CH}), 125.4(\mathrm{~d}, J=12.3 \mathrm{~Hz}, \mathrm{CH}), 131.4(\mathrm{~d}, J$ $=12.3 \mathrm{~Hz}, C \mathrm{H}), 131.6(\mathrm{~d}, J=1.9 \mathrm{~Hz}, C \mathrm{H}), 132.6(\mathrm{~d}, J=123.8 \mathrm{~Hz}, C), 133.4(\mathrm{~d}, J=9.5 \mathrm{~Hz}, C \mathrm{H})$, $141.5(\mathrm{~d}, J=10.3 \mathrm{~Hz}, C), 196.3(\mathrm{~d}, J=11.3 \mathrm{~Hz}, C) .{ }^{31} \mathrm{P}\left\{{ }^{1} \mathrm{H}\right\}$ NMR (159 MHz, $\left.\mathrm{CDCl}_{3}, \delta\right): 20.3$. HRMS$\mathrm{EI}(\mathrm{m} / \mathrm{z})$ : $[\mathrm{M}]^{+}$calcd for $\mathrm{C}_{22} \mathrm{H}_{28} \mathrm{NOP}, 353.1909$; found, 353.1903. mp 95-100 ${ }^{\circ} \mathrm{C}$. IR (neat, $\mathrm{cm}^{-1}$ ): 1186 $(\mathrm{P}=\mathrm{O}), 1652(\mathrm{C}=\mathrm{N})$.

(E)-N-(3-Methylbutan-2-ylidene)-P,P-di-o-tolylphosphinic amide (1d).

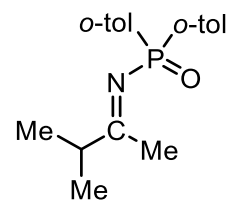

1d

1d was prepared from the corresponding nitrile according to procedure A. The product $1 \mathbf{d}$ was obtained in $9 \%$ yield ( $146.2 \mathrm{mg}, 0.45 \mathrm{mmol}$, white solid) from the corresponding nitrile (5.0 mmol).

${ }^{1} \mathrm{H} \mathrm{NMR}\left(392 \mathrm{MHz}, \mathrm{CDCl}_{3}, \delta\right): 1.17$ (d, $\left.J=6.7 \mathrm{~Hz}, 6 \mathrm{H}\right), 2.30$ (s, 6H), 2.42 (d, $\left.J=2.0 \mathrm{~Hz}, 3 \mathrm{H}\right), 2.72$ (h, $J=6.9 \mathrm{~Hz}, 1 \mathrm{H}), 7.14-7.17$ (m, 2H), 7.26-7.30 (m, 2H), 7.35-7.39 (m, 2H), 8.09 (ddd, $J=1.4,5.9$, $13.7 \mathrm{~Hz}, 2 \mathrm{H}) .{ }^{13} \mathrm{C} \mathrm{NMR}\left(99 \mathrm{MHz}, \mathrm{CDCl}_{3}, \delta\right): 19.9\left(\mathrm{CH}_{3}\right), 21.6\left(\mathrm{~d}, J=3.8 \mathrm{~Hz}, C \mathrm{H}_{3}\right), 24.9(\mathrm{~d}, J=14.2$ Hz, $\left.C_{3}\right), 42.4$ (d, $\left.J=20.8 \mathrm{~Hz}, C H\right), 125.4(\mathrm{~d}, J=11.3 \mathrm{~Hz}, C \mathrm{H}), 131.4$ (d, $\left.J=12.3 \mathrm{~Hz}, C \mathrm{H}\right), 131.6$ (d, $J=1.9 \mathrm{~Hz}, C \mathrm{H}), 132.5$ (d, $J=124.6 \mathrm{~Hz}, C), 133.4(\mathrm{~d}, J=8.5 \mathrm{~Hz}, C \mathrm{H}), 141.5(\mathrm{~d}, J=10.4 \mathrm{~Hz}, C)$, 196.9 (d, $J=11.4 \mathrm{~Hz}, C) .{ }^{31} \mathrm{P}\left\{{ }^{1} \mathrm{H}\right\}$ NMR $\left(159 \mathrm{MHz}, \mathrm{CDCl}_{3}, \delta\right): 20.4$. HRMS-EI $(\mathrm{m} / \mathrm{z})$ : $[\mathrm{M}]^{+}$calcd for $\mathrm{C}_{19} \mathrm{H}_{24} \mathrm{NOP}, 313.1596$; found, 313.1593. $\mathrm{mp} 108-111^{\circ} \mathrm{C}$. IR (neat, $\left.\mathrm{cm}^{-1}\right)$ : $1188(\mathrm{P}=\mathrm{O}), 1658(\mathrm{C}=\mathrm{N})$.

\section{(E)-N-(3,3-Dimethylbutan-2-ylidene)-P,P-di-o-tolylphosphinic amide (1e).}

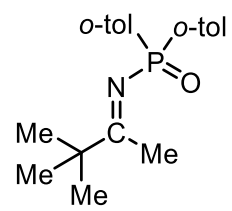

$1 e$

1e was prepared from the corresponding nitrile according to procedure A. The product 1e was obtained in 32\% yield (414.6 mg, $1.27 \mathrm{mmol}$, white solid) from the corresponding nitrile (4.0 mmol). The spectroscopic data were matched in those reported. ${ }^{5}$

${ }^{1} \mathrm{H}$ NMR (392 MHz, $\mathrm{CDCl}_{3}, \delta$ ): 1.22 (s, 9H), 2.28 (s, 6H), 2.43 (d, $\left.J=1.8 \mathrm{~Hz}, 3 \mathrm{H}\right), 7.13-7.17$ (m, 2H), 7.26-7.30 (m, 2H), 7.35-7.39 (m, 2H), 8.09 (dd, $J=1.8,7.6 \mathrm{~Hz}, 2 \mathrm{H}), 8.12$ (dd, $J=1.4,7.6 \mathrm{~Hz}$, 2H). HRMS-EI $(\mathrm{m} / \mathrm{z})$ : $[\mathrm{M}]^{+}$calcd for $\mathrm{C}_{20} \mathrm{H}_{26} \mathrm{NOP}, 325.1752$; found, 327.1746 . 
(E)-N-(3,3-Dimethylhexan-2-ylidene)-P,P-diphenylphosphinic amide (1f).

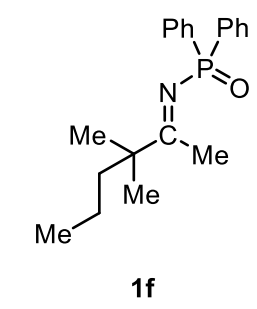

1f was prepared from the corresponding nitrile according to procedure A. The product $\mathbf{1 f}$ was obtained in $44 \%$ yield $(579.3 \mathrm{mg}, 1.77 \mathrm{mmol}$, white solid) from the corresponding nitrile (4.0 mmol).

${ }^{1} \mathrm{H} \mathrm{NMR}\left(392 \mathrm{MHz}, \mathrm{CDCl}_{3}, \delta\right): 0.84$ (t, $\left.J=7.4 \mathrm{~Hz}, 3 \mathrm{H}\right), 1.09-1.18(\mathrm{~m}, 2 \mathrm{H}), 1.20$ (s, 6H), 1.54-1.58 (m, 2H), $2.46(\mathrm{~d}, J=1.6 \mathrm{~Hz}, 3 \mathrm{H}), 7.38-7.47(\mathrm{~m}, 6 \mathrm{H}), 7.89-7.94(\mathrm{~m}, 4 \mathrm{H}) .{ }^{13} \mathrm{C} \mathrm{NMR}\left(100 \mathrm{MHz}, \mathrm{CDCl}_{3}\right.$,

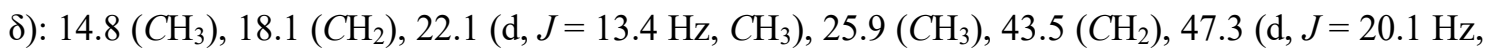
C), 128.4 (d, $J=12.5 \mathrm{~Hz}, C \mathrm{H}), 131.3(\mathrm{~d}, J=2.9 \mathrm{~Hz}, C \mathrm{H}), 131.6$ (d, $J=9.6 \mathrm{~Hz}, C \mathrm{H}), 135.1$ (d, $J=$ 131.2 Hz, C), 197.7 (d, $J=12.4 \mathrm{~Hz}, C) .{ }^{31} \mathrm{P}\left\{{ }^{1} \mathrm{H}\right\}$ NMR (159 MHz, $\left.\mathrm{CDCl}_{3}, \delta\right): 17.3$. HRMS-ESI $(\mathrm{m} / \mathrm{z})$ : $[\mathrm{M}+\mathrm{H}]^{+}$calcd for $\mathrm{C}_{20} \mathrm{H}_{27} \mathrm{NOP}, 328.1825$; found, 328.1823. mp 56-61 ${ }^{\circ} \mathrm{C}$. IR (neat, $\mathrm{cm}^{-1}$ ): $1203(\mathrm{P}=\mathrm{O})$, $1650(\mathrm{C}=\mathrm{N})$.

(E)-P,P-Diphenyl- $N$-[1-(1-phenylcyclobutyl)ethylidene]phosphinic amide (1g).

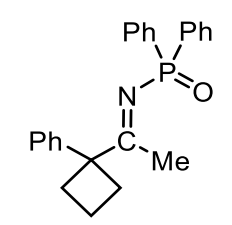

19

$1 \mathrm{~g}$ was prepared from the corresponding nitrile according to procedure A. The product $1 \mathrm{~g}$ was obtained in $28 \%$ yield $(416.6 \mathrm{mg}, 1.12 \mathrm{mmol}$, white solid) from the corresponding nitrile (4.0 mmol).

${ }^{1} \mathrm{H} \mathrm{NMR}\left(392 \mathrm{MHz}, \mathrm{CDCl}_{3}, \delta\right.$ ): 1.89 (quint, $J=13.2 \mathrm{~Hz}, 2 \mathrm{H}$ ), 2.23 (d, $J=2.0 \mathrm{~Hz}, 3 \mathrm{H}$ ), 2.50-2.57 (m, 2H), 2.83-2.90 (m, 2H), 7.17-7.31 (m, 5H), 7.42-7.50 (m, 6H), 7.96 (ddd, $J=1.5,5.9,9.8 \mathrm{~Hz}$, 4H). ${ }^{13} \mathrm{C} \mathrm{NMR}\left(100 \mathrm{MHz}, \mathrm{CDCl}_{3}, \delta\right): 15.9\left(\mathrm{CH}_{2}\right), 21.9\left(\mathrm{~d}, J=12.4 \mathrm{~Hz}, \mathrm{CH}_{3}\right), 31.9\left(\mathrm{CH}_{2}\right), 59.1(\mathrm{~d}, J=$ $22.1 \mathrm{~Hz}, C), 126.4(\mathrm{CH}), 126.8(\mathrm{CH}), 128.4(\mathrm{CH}), 128.6(\mathrm{~d}, J=8.6 \mathrm{~Hz}, C \mathrm{H}), 131.5$ (d, $J=2.8 \mathrm{~Hz}$, $C \mathrm{H}), 131.6$ (d, $J=8.6 \mathrm{~Hz}, C \mathrm{H}), 134.9$ (d, $J=130.3 \mathrm{~Hz}, C), 144.0(C), 193.3$ (d, $J=11.5 \mathrm{~Hz}, C$ ). ${ }^{31} \mathrm{P}\left\{{ }^{1} \mathrm{H}\right\}$ NMR (159 MHz, $\left.\mathrm{CDCl}_{3}, \delta\right): 18.5$. HRMS-ESI $(\mathrm{m} / \mathrm{z})$ : $[\mathrm{M}+\mathrm{Na}]^{+}$calcd for $\mathrm{C}_{24} \mathrm{H}_{24} \mathrm{NOPNa}$, 396.1488; found, 396.1486. $\mathrm{mp} 88-96^{\circ} \mathrm{C}$. IR (neat, $\left.\mathrm{cm}^{-1}\right)$ : $1204(\mathrm{P}=\mathrm{O}), 1655(\mathrm{C}=\mathrm{N})$. 
(E)-P,P-Diphenyl-N-[1-(1-phenylcyclohexyl)ethylidene]phosphinic amide (1h).

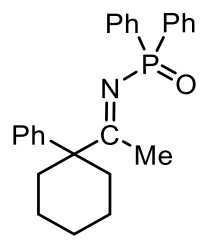

$1 \mathrm{~h}$

1h was prepared from the corresponding nitrile according to procedure A. The product $\mathbf{1 h}$ was obtained in $35 \%$ yield $(567.9 \mathrm{mg}, 1.41 \mathrm{mmol}$, white solid) from the corresponding nitrile (4.0 mmol).

${ }^{1} \mathrm{H}$ NMR (396 MHz, $\left.\mathrm{CDCl}_{3}, \delta\right): 1.37-1.58$ (m, 6H), 2.01-2.08 (m, 2H), 2.24 (d, $\left.J=2.4 \mathrm{~Hz}, 3 \mathrm{H}\right)$, 2.31-2.35 (m, 2H), 7.16-7.30 (m, 5H), 7.42-7.52 (m, 6H), 7.92-7.98 (m, 4H). ${ }^{13} \mathrm{C}$ NMR (100 MHz, $\left.\mathrm{CDCl}_{3}, \delta\right): 22.9\left(\mathrm{~d}, J=13.4 \mathrm{~Hz}, \mathrm{CH}_{3}\right), 23.0\left(\mathrm{CH}_{2}\right), 26.1\left(\mathrm{CH}_{2}\right), 34.4\left(\mathrm{CH}_{2}\right), 54.9(\mathrm{~d}, J=20.1 \mathrm{~Hz}, C)$, $126.8(\mathrm{CH}), 126.9(\mathrm{CH}), 128.5(\mathrm{~d}, J=12.4 \mathrm{~Hz}, C \mathrm{H}), 128.7(C \mathrm{H}), 131.5(\mathrm{~d}, J=2.9 \mathrm{~Hz}, C \mathrm{H}), 131.7$ (d, $J=9.6 \mathrm{~Hz}, C \mathrm{H}), 134.6(\mathrm{~d}, J=130.3 \mathrm{~Hz}, C), 143.5(C), 194.5(\mathrm{~d}, J=12.5 \mathrm{~Hz}, C) .{ }^{31} \mathrm{P}\left\{{ }^{1} \mathrm{H}\right\} \mathrm{NMR}(159$ $\left.\mathrm{MHz}, \mathrm{CDCl}_{3}, \delta\right):$ 18.4. HRMS-EI $(\mathrm{m} / \mathrm{z})$ : [M] $]^{+}$calcd for $\mathrm{C}_{26} \mathrm{H}_{28} \mathrm{NOP}$, 401.1909; found, 401.1897. mp 103-106 ${ }^{\circ} \mathrm{C}$. IR (neat, $\left.\mathrm{cm}^{-1}\right)$ : 1198, $1211(\mathrm{P}=\mathrm{O}), 1651(\mathrm{C}=\mathrm{N})$.

$N-\{(E)-1-[(3 r, 5 r, 7 r)-A d a m a n t a n-1-y l]$ ethylidene $\}-P, P$-diphenylphosphinic amide (1i).

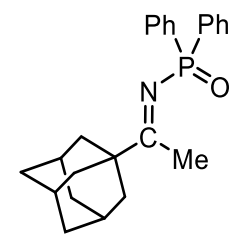

$1 \mathrm{i}$

1i was prepared from the corresponding nitrile according to procedure A. The product $\mathbf{1 i}$ was obtained in 10\% yield $(157.0 \mathrm{mg}, 0.42 \mathrm{mmol}$, white solid) from the corresponding nitrile (4.0 mmol).

${ }^{1} \mathrm{H}$ NMR (392 MHz, $\mathrm{CDCl}_{3}, \delta$ ): 1.75 (q, $\left.J=13.2 \mathrm{~Hz}, 6 \mathrm{H}\right), 1.88$ (d, $\left.J=2.7 \mathrm{~Hz}, 6 \mathrm{H}\right), 2.09$ (s, 3H), $2.45(\mathrm{~d}, J=2.0 \mathrm{~Hz}, 3 \mathrm{H}), 7.38-7.47(\mathrm{~m}, 6 \mathrm{H}), 7.90-7.96(\mathrm{~m}, 4 \mathrm{H}) .{ }^{13} \mathrm{C} \mathrm{NMR}\left(99 \mathrm{MHz}, \mathrm{CDCl}_{3}, \delta\right): 21.2$ (d, $\left.J=13.3 \mathrm{~Hz}, \mathrm{CH}_{3}\right), 28.3(\mathrm{CH}), 36.7\left(\mathrm{CH}_{2}\right), 39.5\left(\mathrm{CH}_{2}\right), 45.9$ (d, $\left.J=19.8 \mathrm{~Hz}, C\right), 128.4(\mathrm{~d}, J=12.3$ Hz, $C \mathrm{H}), 131.2$ (d, $\left.J=2.8 \mathrm{~Hz}, C_{3}\right), 131.6$ (d, $\left.J=9.4 \mathrm{~Hz}, C_{3}\right), 135.3$ (d, $J=130.3 \mathrm{~Hz}, C$ ), 197.9 (d, $J=11.3 \mathrm{~Hz}, C) .{ }^{31} \mathrm{P}\left\{{ }^{1} \mathrm{H}\right\} \mathrm{NMR}\left(159 \mathrm{MHz}, \mathrm{CDCl}_{3}, \delta\right): 17.6$. HRMS-EI $(\mathrm{m} / \mathrm{z})$ : $[\mathrm{M}]^{+}$calcd for $\mathrm{C}_{24} \mathrm{H}_{28} \mathrm{NOP}$, 377.1909; found, 377.1908. $\mathrm{mp} \mathrm{170-172}{ }^{\circ} \mathrm{C}$. IR (neat, $\left.\mathrm{cm}^{-1}\right)$ : $1202(\mathrm{P}=\mathrm{O}), 1651(\mathrm{C}=\mathrm{N})$. 
(E)-N-(2,2-Dimethylheptan-3-ylidene)-P,P-diphenylphosphinic amide (1k).

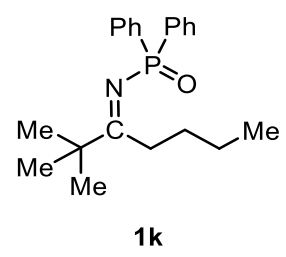

$\mathbf{1 k}$ was prepared from the corresponding nitrile according to the procedure A. The product $\mathbf{1 k}$ was obtained in $66 \%$ yield $(905.7 \mathrm{mg}, 2.65 \mathrm{mmol}$, white solid) from the corresponding nitrile $(4.0 \mathrm{mmol})$.

${ }^{1} \mathrm{H} \mathrm{NMR}\left(392 \mathrm{MHz}, \mathrm{CDCl}_{3}, \delta\right): 0.86(\mathrm{t}, J=7.1 \mathrm{~Hz}, 3 \mathrm{H}), 1.26(\mathrm{~s}, 9 \mathrm{H}), 1.38$ (quint, $\left.J=7.3 \mathrm{~Hz}, 2 \mathrm{H}\right)$, 1.45-1.53 (m, 2H), 2.80-2.84 (m, 2H), 7.37-7.46 (m, 6H), 7.91-7.96 (m, 4H). ${ }^{13} \mathrm{C}$ NMR (100 MHz, $\left.\mathrm{CDCl}_{3}, \delta\right): 13.7\left(\mathrm{CH}_{3}\right), 23.4\left(\mathrm{CH}_{2}\right), 28.0\left(\mathrm{CH}_{3}\right), 31.3\left(\mathrm{CH}_{2}\right), 35.3\left(\mathrm{~d}, J=11.5 \mathrm{~Hz}, \mathrm{CH}_{2}\right), 44.2(\mathrm{~d}, J=$ $21.1 \mathrm{~Hz}, C), 128.4$ (d, $J=12.4 \mathrm{~Hz}, C \mathrm{H}), 131.2$ (d, $J=2.9 \mathrm{~Hz}, C H), 131.6(\mathrm{~d}, J=8.6 \mathrm{~Hz}, C \mathrm{H}), 135.6$ $(\mathrm{d}, J=132.2 \mathrm{~Hz}, C), 200.8(\mathrm{~d}, J=12.4 \mathrm{~Hz}, C) .{ }^{31} \mathrm{P}\left\{{ }^{1} \mathrm{H}\right\} \mathrm{NMR}\left(159 \mathrm{MHz}, \mathrm{CDCl}_{3}, \delta\right): 15.6 . \mathrm{HRMS}-\mathrm{EI}$ (m/z): $[\mathrm{M}]^{+}$calcd for $\mathrm{C}_{21} \mathrm{H}_{28} \mathrm{NOP}, 341.1909$; found, 341.1912. mp 67-70 ${ }^{\circ} \mathrm{C}$. IR (neat, $\mathrm{cm}^{-1}$ ): 1199 $(\mathrm{P}=\mathrm{O}), 1650(\mathrm{C}=\mathrm{N})$.

(E)-N-(4-Methylpentan-2-ylidene)-P,P-diphenylphosphinic amide (11).

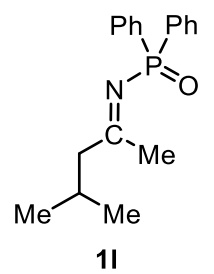

11 was prepared from the corresponding nitrile according to procedure A. The product $\mathbf{1 l}$ was obtained in $25 \%$ yield $(302.0 \mathrm{mg}, 1.0 \mathrm{mmol}$, white solid) from the corresponding nitrile (4.0 mmol).

${ }^{1} \mathrm{H}$ NMR (392 MHz, $\left.\mathrm{CDCl}_{3}, \delta\right): 0.94$ (d, $\left.J=7.1 \mathrm{~Hz}, 6 \mathrm{H}\right), 2.17-2.31$ (m, 1H), 2.43-2.45 (m, 5H), 7.39-7.48 (m, 6H), 7.89-7.95 (m, 4H). $\left.{ }^{13} \mathrm{C} \mathrm{NMR} \mathrm{(100} \mathrm{MHz,} \mathrm{CDCl}_{3}, \delta\right): 22.7\left(\mathrm{CH}_{3}\right), 26.3(C \mathrm{H}), 27.6$ $\left(\mathrm{d}, J=15.4 \mathrm{~Hz}, C \mathrm{H}_{3}\right), 53.2\left(\mathrm{~d}, J=28.1 \mathrm{~Hz}, C \mathrm{H}_{2}\right), 128.4(\mathrm{~d}, J=12.4 \mathrm{~Hz}, C \mathrm{H}), 131.4(\mathrm{~d}, J=2.0 \mathrm{~Hz}$, $C \mathrm{H}), 131.7$ (d, $J=9.5 \mathrm{~Hz}, C \mathrm{H}), 134.9$ (d, $J=130.3 \mathrm{~Hz}, C), 191.9$ (d, $J=10.6 \mathrm{~Hz}, C) .{ }^{31} \mathrm{P}\left\{{ }^{1} \mathrm{H}\right\} \mathrm{NMR}$ (162 $\left.\mathrm{MHz}, \mathrm{CDCl}_{3}, \delta\right): 22.3$. HRMS-EI $(\mathrm{m} / z)$ : $[\mathrm{M}]^{+}$calcd for $\mathrm{C}_{18} \mathrm{H}_{22} \mathrm{NOP}, 299.1439$; found, 299.1431 . mp 166-168 ${ }^{\circ} \mathrm{C}$. IR (neat, $\left.\mathrm{cm}^{-1}\right): 1180(\mathrm{P}=\mathrm{O}), 1555(\mathrm{C}=\mathrm{N})$. 


\section{Procedure B.}

(E)-N-(1-Cyclopropylethylidene)-P,P-diphenylphosphinic amide (1j).
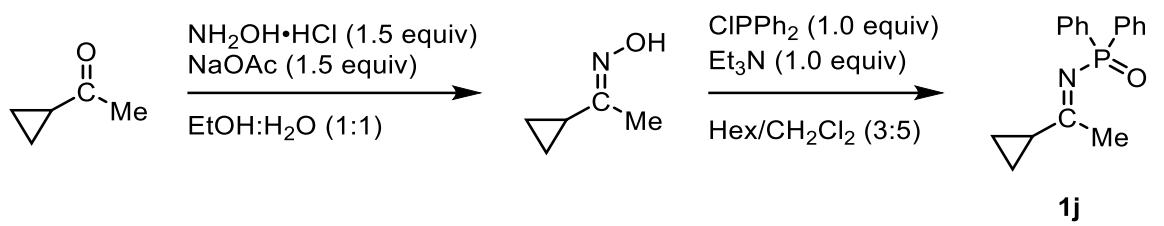

1-Cyclopropylethan-1-one (841.0 mg, $10 \mathrm{mmol}, 1.0$ equiv) was added to a stirred solution of $\mathrm{NH}_{2} \mathrm{OH} \cdot \mathrm{HCl}$ (1.04 g, $15 \mathrm{mmol}, 1.5$ equiv) and $\mathrm{NaOAc}\left(1.23 \mathrm{~g}, 15 \mathrm{mmol}, 1.5\right.$ equiv) in EtOH/ $\mathrm{H}_{2} \mathrm{O}$ $(1 / 1,10 \mathrm{~mL})$, and then heated to reflux. When the reaction was completed, the mixture was cooled down to ambient temperature. The reaction mixture was then diluted with $\mathrm{Na}_{2} \mathrm{CO}_{3}$ saturated aqueous solution and extracted with $\mathrm{CH}_{2} \mathrm{Cl}_{2}$ three times, and dried over $\mathrm{MgSO}_{4}$. After filtration, the obtained oxime was dried under vacuum overnight and used directly for the next process without purification. A solution of chlorodiphenylphosphine $\left(1.04 \mathrm{~mL}, 6.0 \mathrm{mmol}, 1.0\right.$ equiv) in $\mathrm{CH}_{2} \mathrm{Cl}_{2}(6 \mathrm{~mL})$ was added to a stirred solution of the oxime $(597.5 \mathrm{mg}, 6.0 \mathrm{mmol}, 1.0$ equiv) and triethylamine $(836 \mu \mathrm{L}, 6.0$ mmol, 1.0 equiv) in petroleum ether $/ \mathrm{CH}_{2} \mathrm{Cl}_{2}(1: 1,9 \mathrm{~mL})$ over $1 \mathrm{~h}$ at $-45^{\circ} \mathrm{C}$. After the addition was finished, the cooling bath was removed, and the reaction temperature was gradually increased. The mixture was then allowed to stir overnight at ambient temperature. The mixture was directly filtered through a short silica-gel column with EtOAc as an eluent; then the resultant solution was concentrated under reduced pressure. The residue was purified by GPC to afford the corresponding ketimine $\mathbf{1 j}$ (130.2 $\mathrm{mg}, 0.46 \mathrm{mmol}, 8 \%$ yield) as a white solid.

${ }^{1} \mathrm{H}$ NMR (392 MHz, $\left.\mathrm{CDCl}_{3}, \delta\right):$ 0.99-1.04 (m, 2H), 1.15-1.18 (m, 2H), 1.93-2.00 (m, 1H), 2.54 (d, $J=2.0 \mathrm{~Hz}, 3 \mathrm{H}), 7.38-7.45(\mathrm{~m}, 6 \mathrm{H}), 7.83-7.89(\mathrm{~m}, 4 \mathrm{H}) .{ }^{13} \mathrm{C} \mathrm{NMR}\left(99 \mathrm{MHz}, \mathrm{CDCl}_{3}, \delta\right): 12.3\left(\mathrm{CH}_{2}\right)$, $22.7(\mathrm{~d}, J=24.6 \mathrm{~Hz}, C \mathrm{H}), 26.9\left(\mathrm{~d}, J=13.3 \mathrm{~Hz}, C \mathrm{H}_{3}\right), 128.3(\mathrm{~d}, J=12.3 \mathrm{~Hz}, C \mathrm{H}), 131.2(\mathrm{~d}, J=1.9$ $\mathrm{Hz}, C \mathrm{H}), 131.4(\mathrm{~d}, J=8.5 \mathrm{~Hz}, C \mathrm{H}), 135.1$ (d, $J=131.3 \mathrm{~Hz}, C), 194.1(\mathrm{~d}, J=7.5 \mathrm{~Hz}, C) .{ }^{31} \mathrm{P}\left\{{ }^{1} \mathrm{H}\right\} \mathrm{NMR}$ (162 MHz, $\left.\mathrm{CDCl}_{3}, \delta\right):$ 18.4. HRMS-EI $(\mathrm{m} / z)$ : $[\mathrm{M}]^{+}$calcd for $\mathrm{C}_{17} \mathrm{H}_{18} \mathrm{NOP}, 283.1126$; found, 283.1120. mp 118-122 ${ }^{\circ} \mathrm{C}$. IR (neat, $\left.\mathrm{cm}^{-1}\right): 1183,1194(\mathrm{P}=\mathrm{O}), 1652(\mathrm{C}=\mathrm{N})$. 
(E)-P,P-Diphenyl-N-(4-phenylbutan-2-ylidene)phosphinic amide (1m).

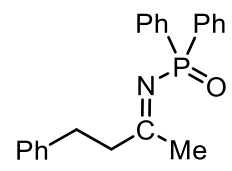

$1 \mathrm{~m}$

$1 \mathbf{m}$ was prepared from the corresponding ketone according to procedure $\mathrm{B}$. The product $\mathbf{1} \mathbf{m}$ was obtained in $7 \%$ yield $(127.7 \mathrm{mg}, 0.37 \mathrm{mmol}$, white solid) from the corresponding oxime (5.0 mmol). The spectroscopic data were matched in those reported ${ }^{6}$.

${ }^{1} \mathrm{H}$ NMR (392 MHz, $\left.\mathrm{CDCl}_{3}, \delta\right): 2.47$ (d, $\left.J=1.6 \mathrm{~Hz}, 3 \mathrm{H}\right), 2.90$ (t, $\left.J=7.3 \mathrm{~Hz}, 2 \mathrm{H}\right), 3.04$ (t, $J=7.4$ $\mathrm{Hz}, 2 \mathrm{H}), 7.19-7.28$ (m, 5H), 7.38-7.50 (m, 6H), 7.83-7.88 (m, 4H). ${ }^{13} \mathrm{C} \mathrm{NMR}\left(99 \mathrm{MHz}, \mathrm{CDCl}_{3}, \delta\right)$ : $27.3\left(\mathrm{~d}, J=14.2 \mathrm{~Hz}, \mathrm{CH}_{3}\right), 31.7\left(\mathrm{CH}_{2}\right), 45.1\left(\mathrm{~d}, J=20.7 \mathrm{~Hz}, \mathrm{CH}_{2}\right), 126.2(\mathrm{CH}), 128.31(\mathrm{CH}), 128.33$ $(C H), 128.5(\mathrm{~d}, J=11.4 \mathrm{~Hz}, C \mathrm{H}), 131.4(\mathrm{~d}, J=2.8 \mathrm{~Hz}, C \mathrm{H}), 131.6(\mathrm{~d}, J=9.4 \mathrm{~Hz}, C \mathrm{H}), 134.6(\mathrm{~d}, J=$ $130.3 \mathrm{~Hz}, C), 140.1(C), 190.8(\mathrm{~d}, J=10.3 \mathrm{~Hz}, C)$. HRMS-EI $(\mathrm{m} / \mathrm{z})$ : [M] ${ }^{+}$calcd for $\mathrm{C}_{22} \mathrm{H}_{22} \mathrm{NOP}$, 347.1439; found, 347.1430 .

(E)-N-(Butan-2-ylidene)-P,P-diphenylphosphinic amide (1n).<smiles>COC(=NP(=O)(c1ccccc1)c1ccccc1)OC</smiles>

1n was prepared from the corresponding ketone according to procedure B. The product $1 \mathbf{n}$ was obtained in $5 \%$ yield (102.8 $\mathrm{mg}, 0.38 \mathrm{mmol}$, white solid) from the corresponding oxime $(8.0 \mathrm{mmol})$.

${ }^{1} \mathrm{H}$ NMR $\left(392 \mathrm{MHz}, \mathrm{CDCl}_{3}, \delta\right): 1.20(\mathrm{t}, J=7.3 \mathrm{~Hz}, 3 \mathrm{H}), 2.46(\mathrm{~d}, J=2.0 \mathrm{~Hz}, 3 \mathrm{H}), 2.58$ (q, $J=7.3$ $\mathrm{Hz}, 2 \mathrm{H}), 7.39-7.48(\mathrm{~m}, 6 \mathrm{H}), 7.90-7.96(\mathrm{~m}, 4 \mathrm{H}) .{ }^{13} \mathrm{C} \mathrm{NMR}$ (99 MHz, $\left.\mathrm{CDCl}_{3}, \delta\right): 10.2\left(\mathrm{CH}_{3}\right), 26.9(\mathrm{~d}$, $\left.J=14.2 \mathrm{~Hz}, C_{3}\right), 37.4\left(\mathrm{~d}, J=20.8 \mathrm{~Hz}, C \mathrm{H}_{2}\right), 128.5(\mathrm{~d}, J=12.3 \mathrm{~Hz}, C \mathrm{H}), 131.4(\mathrm{~d}, J=2.9 \mathrm{~Hz}, C \mathrm{H})$, 131.7 (d, $J=9.5 \mathrm{~Hz}, C \mathrm{H}), 135.0$ (d, $J=131.3 \mathrm{~Hz}, C), 192.8$ (d, $J=10.4 \mathrm{~Hz}, C) .{ }^{31} \mathrm{P}\left\{{ }^{1} \mathrm{H}\right\} \mathrm{NMR}(162$ $\left.\mathrm{MHz}, \mathrm{CDCl}_{3}, \delta\right):$ 22.4. HRMS-EI $(\mathrm{m} / \mathrm{z})$ : $[\mathrm{M}]^{+}$calcd for $\mathrm{C}_{16} \mathrm{H}_{18} \mathrm{NOP}$, 271.1126; found, 271.1121. mp 161-168 ${ }^{\circ} \mathrm{C}$. IR (neat, $\left.\mathrm{cm}^{-1}\right): 1179(\mathrm{P}=\mathrm{O}), 1557(\mathrm{C}=\mathrm{N})$. 


\section{General Experimental Procedures for Borylation of Ketimines}

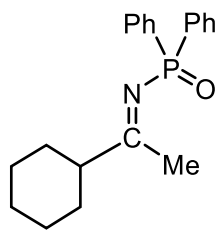

1 a

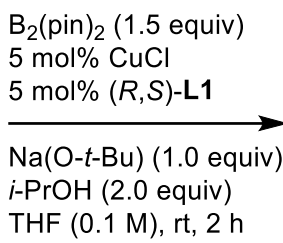

$\operatorname{THF}(0.1 \mathrm{M}), \mathrm{rt}, 2 \mathrm{~h}$

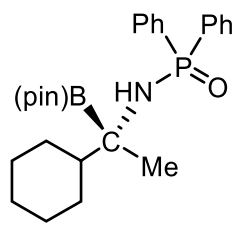

$3 a$

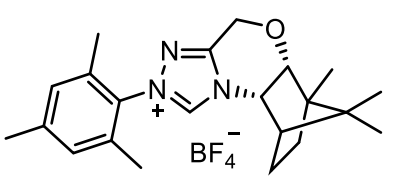

$(R, S)-\mathbf{L} 1$

$\mathrm{CuCl}(1.0 \mathrm{mg}, 0.01 \mathrm{mmol}, 5 \mathrm{~mol} \%),(R, S)-\mathbf{L 1}(4.4 \mathrm{mg}, 0.01 \mathrm{mmol}, 5 \mathrm{~mol} \%$, and bis(pinacolato)diboron $(76.2 \mathrm{mg}, 0.30 \mathrm{mmol}, 1.5$ equiv) were placed in a vial with a screw cap containing a Teflon ${ }^{\circledR}$-coated rubber septum under air. The vial was put in a glove box, and then $\mathrm{Na}(\mathrm{O}$ $t$-Bu) (19.2 mg, $0.20 \mathrm{mmol}, 1.0$ equiv) was also added to the vial in the glove box under an argon atmosphere. After the reaction, the vial was removed from the glove box, THF $(0.6 \mathrm{~mL})$ was added to the vial via a syringe. The resulting mixture was stirred for $10 \mathrm{~min}$ at room temperature, then a THF solution $(1.4 \mathrm{~mL})$ of ketimine $1 \mathrm{a}(65.0 \mathrm{mg}, 0.20 \mathrm{mmol}, 1.0$ equiv) was added dropwise to the vial. $i$ PrOH $(31 \mu \mathrm{L}, 0.40 \mathrm{mmol}, 2.0$ equiv) was added to the reaction mixture. After the resulting mixture was stirred at room temperature for $2 \mathrm{~h}$, the reaction mixture was analyzed by GC to check the completeness of the reaction. The mixture was directly filtered through a short silica-gel column with EtOAc as an eluent; then the resultant solution was concentrated under reduced pressure. The crude product was purified by silica-gel column chromatography with hexane/EtOAc eluent (100:0 to 40:60). After silica-gel column purification, the mixture was further purified by extraction with $\mathrm{CH}_{2} \mathrm{Cl}_{2}$ and $\mathrm{H}_{2} \mathrm{O}$ three times to remove pinacol. The $\mathrm{CH}_{2} \mathrm{Cl}_{2}$ solution was dried over $\mathrm{MgSO}_{4}$. After filtration, the corresponding product 3a was obtained in $86 \%$ yield $(78.0 \mathrm{mg}, 0.17 \mathrm{mmol})$ as a colorless oil. The racemic sample was prepared using $\mathrm{ICy} \cdot \mathrm{HCl}$ for the ligand instead of $(R, S)-\mathbf{L 1}$. 


\section{(R)- $N$-[1-Cyclohexyl-1-(4,4,5,5-tetramethyl-1,3,2-dioxaborolan-2-yl)ethyl]-P,P-}

diphenylphosphinic amide $[(R)-3 a]$.

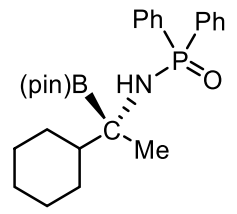

(R)-3a

The reaction was conducted with $65.1 \mathrm{mg}(0.20 \mathrm{mmol})$ of 1a. The product $(R)-\mathbf{3 a}$ was purified by flash column chromatography $\left(\mathrm{SiO}_{2}\right.$, hexane/EtOAc, 100:0 $\left.\rightarrow 40: 60\right)$ and extracted with $\mathrm{H}_{2} \mathrm{O}$ to remove pinacol, and dried over $\mathrm{MgSO}_{4}$. After filtration, $(R)$-3a was obtained in $86 \%$ yield $(78.0 \mathrm{mg}$, $0.17 \mathrm{mmol}$, colorless oil) with $89 \%$ ee.

${ }^{1} \mathrm{H}$ NMR (396 MHz, $\left.\mathrm{CDCl}_{3}, \delta\right): 1.01-1.26(\mathrm{~m}, 5 \mathrm{H}), 1.17$ (s, 3H), 1.29 (s, 6H) $1.30(\mathrm{~s}, 6 \mathrm{H}), 1.44-$ $1.50(\mathrm{~m}, 1 \mathrm{H}), 1.64-1.89(\mathrm{~m}, 5 \mathrm{H}), 3.04(\mathrm{~d}, J=7.9 \mathrm{~Hz}, 1 \mathrm{H}), 7.38-7.48(\mathrm{~m}, 6 \mathrm{H}), 7.80-7.88(\mathrm{~m}, 4 \mathrm{H}) .{ }^{13} \mathrm{C}$ NMR (99 MHz, $\left.\mathrm{CDCl}_{3}, \delta\right): 19.6\left(\mathrm{~d}, J=3.9 \mathrm{~Hz}, \mathrm{CH}_{3}\right), 25.0\left(\mathrm{CH}_{3}\right), 25.1\left(\mathrm{CH}_{3}\right), 26.76\left(\mathrm{CH}_{2}\right), 26.81(\mathrm{~d}$, $\left.J=1.9 \mathrm{~Hz}, \mathrm{CH}_{2}\right), 28.5\left(\mathrm{~d}, J=42.6 \mathrm{~Hz}, C \mathrm{H}_{2}\right), 47.5(\mathrm{~d}, J=4.4 \mathrm{~Hz}, C \mathrm{H}), 49.5$ (br, B-C), $84.2(C), 128.2$ $(C \mathrm{H}), 128.4(\mathrm{CH}), 131.3(\mathrm{CH}), 131.8(\mathrm{~d}, J=9.5 \mathrm{~Hz}, C \mathrm{H}), 132.0(\mathrm{~d}, J=9.4 \mathrm{~Hz}, C \mathrm{H}), 135.3(\mathrm{~d}, J=6.6$ $\mathrm{Hz}, C), 136.6(\mathrm{~d}, J=5.7 \mathrm{~Hz}, C) .{ }^{11} \mathrm{~B}\left\{{ }^{1} \mathrm{H}\right\}$ NMR $\left(127 \mathrm{MHz}, \mathrm{CDCl}_{3}, \delta\right): 33.1 .{ }^{31} \mathrm{P}\left\{{ }^{1} \mathrm{H}\right\} \mathrm{NMR}(159 \mathrm{MHz}$, $\left.\mathrm{CDCl}_{3}, \delta\right)$ : 21.9. HRMS-ESI $(\mathrm{m} / z)$ : $[\mathrm{M}+\mathrm{Na}]^{+}$calcd for $\mathrm{C}_{26} \mathrm{H}_{37}{ }^{11} \mathrm{BNO}_{3} \mathrm{PNa}, 476.2501$; found, 476.2496. $[\alpha]_{\mathrm{D}}^{21.0}-6.59$ (c 1.10 in $\mathrm{CHCl}_{3}, 89 \%$ ee). Daicel CHIRALPAK ${ }^{\circledR}$ OZ-3, 2-PrOH/Hexane $=10 / 90,0.5$ $\mathrm{mL} / \mathrm{min}, 40^{\circ} \mathrm{C}, S$ isomer: $t_{S}=11.97 \mathrm{~min}$., $R$ isomer: $t_{R}=15.01 \mathrm{~min}$. IR (neat, $\left.\mathrm{cm}^{-1}\right): 1200(\mathrm{P}=\mathrm{O})$.

\section{(R)- $N$-[1-Cyclohexyl-1-(4,4,5,5-tetramethyl-1,3,2-dioxaborolan-2-yl)ethyl]-P,P-di-m-}

tolylphosphinic amide $[(R)-3 b]$.

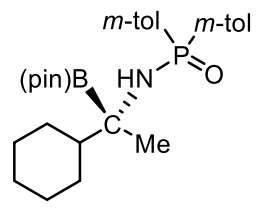

(R)-3b

The reaction was conducted with $71 \mathrm{mg}(0.20 \mathrm{mmol})$ of $\mathbf{1 b}$ for $2 \mathrm{~h}$. The product $(R)-\mathbf{3 b}$ was purified by flash column chromatography $\left(\mathrm{SiO}_{2}\right.$, hexane/EtOAc, 100:0 $\left.\rightarrow 40: 60\right)$ and extracted with $\mathrm{H}_{2} \mathrm{O}$ to remove pinacol, and dried over $\mathrm{MgSO}_{4}$. After filtration, $(R)-\mathbf{3 b}$ was obtained in $74 \%$ yield $(70.6 \mathrm{mg}$, $0.15 \mathrm{mmol}$, colorless oil) with $90 \%$ ee.

${ }^{1} \mathrm{H}$ NMR (392 MHz, $\mathrm{CDCl}_{3}, \delta$ ): 1.01-1.24 (m, 5H), 1.17 (s, 3H), 1.29 (s, 6H), 1.31 (s, 6H), $1.43-$ $1.50(\mathrm{~m}, 1 \mathrm{H}), 1.56-1.94(\mathrm{~m}, 5 \mathrm{H}), 2.36(\mathrm{~s}, 6 \mathrm{H}), 3.02(\mathrm{~d}, J=7.8 \mathrm{~Hz}, 1 \mathrm{H}), 7.26-7.34(\mathrm{~m}, 4 \mathrm{H}), 7.57-7.62$ (m, 2H), $7.70(\mathrm{dd}, J=4.5,12.7 \mathrm{~Hz}, 2 \mathrm{H}) .{ }^{13} \mathrm{C} \mathrm{NMR}\left(100 \mathrm{MHz}, \mathrm{CDCl}_{3}, \delta\right): 19.5\left(\mathrm{~d}, J=3.8 \mathrm{~Hz}, \mathrm{CH}_{3}\right)$, 
$21.5\left(\mathrm{CH}_{3}\right), 25.0\left(\mathrm{CH}_{3}\right), 25.1\left(\mathrm{CH}_{3}\right), 26.76\left(\mathrm{CH}_{2}\right), 26.82\left(\mathrm{~d}, J=2.9 \mathrm{~Hz}, \mathrm{CH}_{2}\right), 28.4(\mathrm{~d}, J=47.9 \mathrm{~Hz}$, $C_{2}$ ), 47.5 (d, $\left.J=3.9 \mathrm{~Hz}, C \mathrm{H}\right), 49.5$ (br, B-C), $84.2(C), 128.1$ (d, $\left.J=3.9 \mathrm{~Hz}, C H\right), 128.2$ (d, $J=3.8$ $\mathrm{Hz}, C \mathrm{H}), 128.8$ (d, $J=10.6 \mathrm{~Hz}, C \mathrm{H}), 128.9$ (d, $J=10.5 \mathrm{~Hz}, C H), 131.98$ (d, $J=2.9 \mathrm{~Hz}, C H), 132.01$ (d, $J=2.8 \mathrm{~Hz}, C \mathrm{H}), 135.2$ (d, $J=13.4 \mathrm{~Hz}, C), 136.5$ (d, $J=11.5 \mathrm{~Hz}, C), 138.0$ (d, $J=12.4 \mathrm{~Hz}, C$ ). ${ }^{11} \mathrm{~B}\left\{{ }^{1} \mathrm{H}\right\}$ NMR (127 MHz, $\left.\mathrm{CDCl}_{3}, \delta\right): 33.5 .{ }^{31} \mathrm{P}\left\{{ }^{1} \mathrm{H}\right\}$ NMR (159 MHz, $\mathrm{CDCl}_{3}, \delta$ ): 22.3. HRMS-ESI $(m / z):[\mathrm{M}+\mathrm{Na}]^{+}$calcd for $\mathrm{C}_{28} \mathrm{H}_{41}{ }^{11} \mathrm{BNO}_{3} \mathrm{PNa}, 504.2814$; found, 504.2814. $[\alpha]_{\mathrm{D}}{ }^{25.1}+1.24$ (c 1.45 in $\mathrm{CHCl}_{3}, 90 \%$ ee). Daicel CHIRALPAK ${ }^{\circledR} \mathrm{OZ}-3,2-\mathrm{PrOH} / \mathrm{Hexane}=10 / 90,0.5 \mathrm{~mL} / \mathrm{min}, 40^{\circ} \mathrm{C}, \mathrm{S}$ isomer: $t_{S}=10.21 \mathrm{~min} ., \quad R$ isomer: $t_{R}=11.23 \mathrm{~min}$. IR (neat, $\left.\mathrm{cm}^{-1}\right): 1193(\mathrm{P}=\mathrm{O})$.

\section{(R)- $N$-[1-Cyclohexyl-1-(4,4,5,5-tetramethyl-1,3,2-dioxaborolan-2-yl)ethyl]-P,P-di-o-}

\section{tolylphosphinic amide $[(R)-3 c]$.}

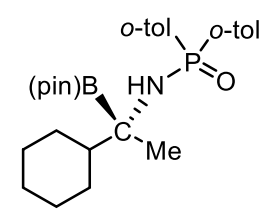

$(R)-3 c$

The reaction was conducted with $69.2 \mathrm{mg}(0.20 \mathrm{mmol})$ of $\mathbf{1 c}$ for $3 \mathrm{~h}$. The product $(R)-\mathbf{3 a}$ was purified by flash column chromatography $\left(\mathrm{SiO}_{2}, \mathrm{CH}_{2} \mathrm{Cl}_{2} / \mathrm{Et}_{2} \mathrm{O}, 100: 0 \rightarrow 80: 20\right)$ and obtained in $92 \%$ yield ( $86.3 \mathrm{mg}, 0.18 \mathrm{mmol}$, colorless oil) with $95 \%$ ee.

${ }^{1} \mathrm{H}$ NMR (396 MHz, CDCl $\left.3, \delta\right): 1.03-1.24$ (m, 5H), 1.26 (s, 6H), 1.28 (s, 6H), 1.34 (s, 3H), 1.60 $1.91(\mathrm{~m}, 6 \mathrm{H}), 2.44(\mathrm{~s}, 3 \mathrm{H}), 2.57$ (s, 3H), $2.80(\mathrm{~d}, J=11.5 \mathrm{~Hz}, 1 \mathrm{H}), 7.14-7.24(\mathrm{~m}, 4 \mathrm{H}), 7.35$ (q, $J=7.8$ $\mathrm{Hz}, 2 \mathrm{H}), 7.53$ (ddd, $J=1.0,7.9,11.9 \mathrm{~Hz}, 1 \mathrm{H}), 7.89$ (ddd, $J=1.2,7.9,15.8 \mathrm{~Hz}, 1 \mathrm{H}) .{ }^{13} \mathrm{C}$ NMR $(99$ $\mathrm{MHz}, \mathrm{CDCl}_{3}, \delta$ ): $20.0\left(\mathrm{~d}, J=2.9 \mathrm{~Hz}, C_{3}\right), 21.7\left(\mathrm{~d}, J=3.8 \mathrm{~Hz}, \mathrm{CH}_{3}\right), 22.0\left(\mathrm{~d}, J=3.8 \mathrm{~Hz}, C_{3}\right), 25.0$ $\left(\mathrm{CH}_{3}\right), 25.1\left(\mathrm{CH}_{3}\right), 26.76\left(\mathrm{CH}_{2}\right), 26.84\left(\mathrm{CH}_{2}\right), 28.7\left(\mathrm{~d}, J=64.3 \mathrm{~Hz}, \mathrm{CH}_{2}\right), 48.1(\mathrm{~d}, J=3.8 \mathrm{~Hz}, \mathrm{CH})$, 49.6 (br, B-C), 84.1 (C), 124.9 (d, $J=12.3 \mathrm{~Hz}, C H), 125.1$ (d, $J=12.3 \mathrm{~Hz}, C H), 131.19$ (d, $J=2.8$ $\mathrm{Hz}, C \mathrm{H}), 131.23$ (d, $J=2.9 \mathrm{~Hz}, C \mathrm{H}), 131.5$ (d, $J=12.3 \mathrm{~Hz}, C \mathrm{H}), 131.7$ (d, $J=11.3 \mathrm{~Hz}, C \mathrm{H}), 132.9$ (d, $J=11.3 \mathrm{~Hz}, C \mathrm{H}), 133.61$ (d, $J=29.2 \mathrm{~Hz}, C), 133.67$ (d, $J=10.4 \mathrm{~Hz}, C \mathrm{H}), 134.8$ (d, $J=32.2 \mathrm{~Hz}$, C), 142.0 (d, $J=9.4 \mathrm{~Hz}, C), 142.1(\mathrm{~d}, J=10.4 \mathrm{~Hz}, C) .{ }^{11} \mathrm{~B}\left\{{ }^{1} \mathrm{H}\right\}$ NMR $\left(127 \mathrm{MHz}, \mathrm{CDCl}_{3}, \delta\right)$ : 33.6. ${ }^{31} \mathrm{P}\left\{{ }^{1} \mathrm{H}\right\}$ NMR (159 MHz, $\mathrm{CDCl}_{3}, \delta$ ): 27.2. HRMS-ESI $(\mathrm{m} / \mathrm{z})$ : $[\mathrm{M}+\mathrm{Na}]^{+}$calcd for $\mathrm{C}_{28} \mathrm{H}_{41}{ }^{11} \mathrm{BNO}_{3} \mathrm{PNa}$, 504.2814; found, 504.2814. $[\alpha]_{\mathrm{D}}{ }^{23.6}-26.5$ ( 0.26 in $\mathrm{CHCl}_{3}, 95 \%$ ee). Daicel CHIRALPAK ${ }^{\circ}$ OZ-3, 2-PrOH/Hexane $=10 / 90,0.5 \mathrm{~mL} / \mathrm{min}, 40{ }^{\circ} \mathrm{C}, S$ isomer: $t_{S}=8.77 \mathrm{~min} ., \quad R$ isomer: $t_{R}=9.57 \mathrm{~min}$. IR (neat, $\left.\mathrm{cm}^{-1}\right)$ : $1192(\mathrm{P}=\mathrm{O})$. 


\section{(R)-N-[3-Methyl-2-(4,4,5,5-tetramethyl-1,3,2-dioxaborolan-2-yl)butan-2-yl]-P,P-di-o-}

tolylphosphinic amide $[(R)-3 d]$.

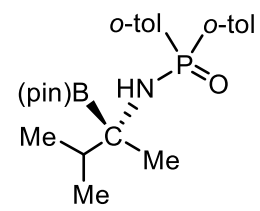

$(R)-3 \mathbf{d}$

The reaction was conducted with $63.7 \mathrm{mg}(0.20 \mathrm{mmol})$ of $\mathbf{1 d}$ for $2 \mathrm{~h}$. The product $(R)-\mathbf{3 d}$ was purified by flash column chromatography $\left(\mathrm{SiO}_{2}, \mathrm{CH}_{2} \mathrm{Cl}_{2} / \mathrm{Et}_{2} \mathrm{O}, 100: 0 \rightarrow 80: 20\right)$ and extracted with $\mathrm{H}_{2} \mathrm{O}$ to remove pinacol, and dried over $\mathrm{MgSO}_{4}$. After filtration, $(R)$-3d was obtained in $91 \%$ yield $(81.8$ $\mathrm{mg}, 0.19 \mathrm{mmol}$, colorless oil) with $97 \%$ ee.

${ }^{1} \mathrm{H}$ NMR (396 MHz, $\left.\mathrm{CDCl}_{3}, \delta\right): 0.98(\mathrm{~d}, J=6.7 \mathrm{~Hz}, 3 \mathrm{H}), 1.01(\mathrm{~d}, J=6.7 \mathrm{~Hz}, 3 \mathrm{H}), 1.25(\mathrm{~s}, 6 \mathrm{H}), 1.27$ (s, 6H), $1.32(\mathrm{~s}, 3 \mathrm{H}), 1.93-2.02(\mathrm{~m}, 1 \mathrm{H}), 2.43(\mathrm{~s}, 3 \mathrm{H}), 2.56$ (s, 3H), 2.75 (d, J=10.7 Hz, 1H), $7.14-$ 7.24 (m, 4H), 7.30-7.39 (m, 2H), 7.56 (ddd, $J=1.4,7.9,11.9 \mathrm{~Hz}, 1 \mathrm{H}), 7.91$ (ddd, $J=1.2,5.9,13.9$ $\mathrm{Hz}, 1 \mathrm{H}) .{ }^{13} \mathrm{C} \mathrm{NMR}\left(100 \mathrm{MHz}, \mathrm{CDCl}_{3}, \delta\right): 18.3\left(\mathrm{CH}_{3}\right), 18.7\left(\mathrm{CH}_{3}\right), 19.7\left(\mathrm{~d}, J=2.9 \mathrm{~Hz}, \mathrm{CH}_{3}\right), 21.8(\mathrm{~d}$, $\left.J=3.9 \mathrm{~Hz}, \mathrm{CH}_{3}\right), 22.0\left(\mathrm{~d}, J=3.8 \mathrm{~Hz}, \mathrm{CH}_{3}\right), 25.0\left(\mathrm{CH}_{3}\right), 25.1\left(\mathrm{CH}_{3}\right), 37.6(\mathrm{~d}, J=4.8 \mathrm{~Hz}, \mathrm{CH}), 49.8$ (br, B-C), $84.1(C), 124.9$ (d, $J=13.4 \mathrm{~Hz}, C \mathrm{H}), 125.1$ (d, $J=13.5 \mathrm{~Hz}, C \mathrm{H}), 131.2$ (d, $J=2.9 \mathrm{~Hz}, C \mathrm{H})$, 131.3 (d, $J=2.0 \mathrm{~Hz}, C \mathrm{H}), 131.5$ (d, $J=11.5 \mathrm{~Hz}, C \mathrm{H}), 131.7$ (d, $J=11.5 \mathrm{~Hz}, C \mathrm{H}), 132.9$ (d, $J=11.5$ Hz, CH), 133.6 (d, $J=25.9 \mathrm{~Hz}, C), 133.7$ (d, $J=10.5 \mathrm{~Hz}, C \mathrm{H}), 134.8$ (d, $J=27.8 \mathrm{~Hz}, C), 141.9$ (d, $J$ $=9.6 \mathrm{~Hz}, C), 142.1(\mathrm{~d}, J=9.6 \mathrm{~Hz}, C) .{ }^{11} \mathrm{~B}\left\{{ }^{1} \mathrm{H}\right\} \operatorname{NMR}\left(127 \mathrm{MHz}, \mathrm{CDCl}_{3}, \delta\right): 33.1 .{ }^{31} \mathrm{P}\left\{{ }^{1} \mathrm{H}\right\} \operatorname{NMR}(159$ $\left.\mathrm{MHz}, \mathrm{CDCl}_{3}, \delta\right):$ 27.2. HRMS-ESI $(\mathrm{m} / \mathrm{z})$ : $[\mathrm{M}+\mathrm{Na}]^{+}$calcd for $\mathrm{C}_{25} \mathrm{H}_{37}{ }^{11} \mathrm{BNO}_{3} \mathrm{PNa}, 464.2501$; found, 464.2501. $[\alpha]_{\mathrm{D}}{ }^{27.4}+1.44\left(c 1.08\right.$ in $\mathrm{CHCl}_{3}, 97 \%$ ee $)$. Daicel CHIRALPAK ${ }^{\circledR}$ OZ-3, 2-PrOH/Hexane $=$ $10 / 90,0.5 \mathrm{~mL} / \mathrm{min}, 40{ }^{\circ} \mathrm{C}, S$ isomer: $t_{S}=9.57 \min ., R$ isomer: $t_{R}=10.16 \min$. IR (neat, $\mathrm{cm}^{-1}$ ): 1191 $(\mathrm{P}=\mathrm{O})$.

(R)- $N$-[3,3-Dimethyl-2-(4,4,5,5-tetramethyl-1,3,2-dioxaborolan-2-yl)butan-2-yl]-P,P-di-otolylphosphinic amide $[(R)-3 e]$.

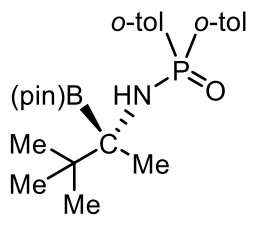

(R)-3e

The reaction was conducted with $63.6 \mathrm{mg}(0.20 \mathrm{mmol})$ of $1 \mathbf{e}$ for $20 \mathrm{~h}$. The product $(R)-3 \mathbf{e}$ was purified by flash column chromatography $\left(\mathrm{SiO}_{2}, \mathrm{CH}_{2} \mathrm{Cl}_{2} / \mathrm{Et}_{2} \mathrm{O}, 100: 0 \rightarrow 80: 20\right)$ and obtained in $85 \%$ yield (74.9 mg, $0.16 \mathrm{mmol}$, white solid) with $99 \%$ ee. 
${ }^{1} \mathrm{H}$ NMR (392 MHz, $\mathrm{CDCl}_{3}, \delta$ ): 1.04 (s, 9H), 1.28 (s, 6H), 1.29 (s, 6H), 1.37 (s, 3H), 2.40 (s, 3H), 2.50 (s, 3H), 2.77 (d, $J=9.4 \mathrm{~Hz}, 1 \mathrm{H}), 7.15-7.24(\mathrm{~m}, 4 \mathrm{H}), 7.34$ (tq, $J=1.4,7.8 \mathrm{~Hz}, 2 \mathrm{H}), 7.66$ (ddd, $J$ $=1.3,7.8,15.7 \mathrm{~Hz}, 1 \mathrm{H}), 7.94(\mathrm{ddd}, J=1.4,7.8,11.8 \mathrm{~Hz}, 1 \mathrm{H}) .{ }^{13} \mathrm{C} \mathrm{NMR}\left(100 \mathrm{MHz}, \mathrm{CDCl}_{3}, \delta\right): 18.0$ (d, $\left.J=2.9 \mathrm{~Hz} \mathrm{CH}_{3}\right), 21.86\left(\mathrm{~d}, J=2.9 \mathrm{~Hz}, \mathrm{CH}_{3}\right), 21.94\left(\mathrm{~d}, J=3.8 \mathrm{~Hz}, \mathrm{CH}_{3}\right), 25.0\left(\mathrm{CH}_{3}\right), 25.2\left(\mathrm{CH}_{3}\right)$, $26.5\left(\mathrm{CH}_{3}\right), 37.2(\mathrm{~d}, J=4.7 \mathrm{~Hz}, C), 52.7$ (br, B-C), $84.2(C), 124.6$ (d $\left.J=12.5 \mathrm{~Hz}, \mathrm{CH}\right), 125.1$ (d, $J=$ 13.4 Hz, CH), 131.1 (d, $J=2.9 \mathrm{~Hz}, C \mathrm{H}), 131.2(\mathrm{~d}, J=2.9 \mathrm{~Hz}, C \mathrm{H}), 131.5(\mathrm{~d}, J=7.7 \mathrm{~Hz}, C \mathrm{H}), 131.6$ (d, $J=7.7 \mathrm{~Hz}, C H), 132.9$ (d, $J=10.5 \mathrm{~Hz}, C H), 133.5$ (d, $J=10.5 \mathrm{~Hz}, C H), 133.9$ (d, $J=28.8 \mathrm{~Hz}, C$ ), 135.1 (d, $J=29.7 \mathrm{~Hz}, C), 141.5(\mathrm{~d}, J=9.5 \mathrm{~Hz}, C), 142.1$ (d, $J=10.6 \mathrm{~Hz}, C) .{ }^{11} \mathrm{~B}\left\{{ }^{1} \mathrm{H}\right\}$ NMR $(127$ $\left.\mathrm{MHz}, \mathrm{CDCl}_{3}, \delta\right): 33.3 .{ }^{31} \mathrm{P}\left\{{ }^{1} \mathrm{H}\right\}$ NMR (159 MHz, $\left.\mathrm{CDCl}_{3}, \delta\right): 26.9$. HRMS-ESI $(\mathrm{m} / \mathrm{z}):[\mathrm{M}+\mathrm{H}]^{+}$calcd for $\mathrm{C}_{26} \mathrm{H}_{40}{ }^{11} \mathrm{BNO}_{3} \mathrm{P}$, 456.2839; found, 456.2831. [ $\left.\alpha\right]_{\mathrm{D}}^{23.3}-11.68$ (c 1.01 in $\mathrm{CHCl}_{3}, 99 \%$ ee). Daicel CHIRALPAK ${ }^{\circledR} \mathrm{IA}-3,2-\mathrm{PrOH} / \mathrm{Hexane}=8 / 92,0.5 \mathrm{~mL} / \mathrm{min}, 40^{\circ} \mathrm{C}, S$ isomer: $t_{S}=48.21 \mathrm{~min} ., 2$ isomer: $t_{R}=51.56 \min . \mathrm{mp} 114-120^{\circ} \mathrm{C}$. IR (neat, $\left.\mathrm{cm}^{-1}\right): 1191(\mathrm{P}=\mathrm{O})$.

\section{(R)-N-[3,3-Dimethyl-2-(4,4,5,5-tetramethyl-1,3,2-dioxaborolan-2-yl)hexan-2-yl]-P,P-}

\section{diphenylphosphinic amide $[(R)-3 f]$.}

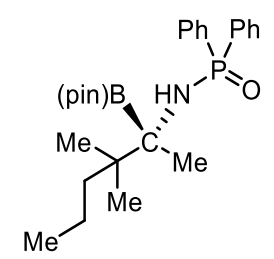

$(R)-3 f$

The reaction was conducted with $64.8 \mathrm{mg}(0.20 \mathrm{mmol})$ of $\mathbf{1 f}$ for $3 \mathrm{~h}$. The product $(R)$-3f was purified by flash column chromatography $\left(\mathrm{SiO}_{2}\right.$, hexane/EtOAc, 100:0 $\left.\rightarrow 50: 50\right)$ and extracted with $\mathrm{H}_{2} \mathrm{O}$ to remove pinacol, and dried over $\mathrm{MgSO}_{4}$. After filtration, $(R)-3 \mathbf{f}$ was obtained in $82 \%$ yield $(73.9 \mathrm{mg}$, $0.16 \mathrm{mmol}$, colorless oil) with $99 \%$ ee.

${ }^{1} \mathrm{H}$ NMR $\left(392 \mathrm{MHz}, \mathrm{CDCl}_{3}, \delta\right): 0.91$ (t, $\left.J=6.9 \mathrm{~Hz}, 3 \mathrm{H}\right), 0.955$ (s, 3H), 0.961 (s, 3H), 1.17 (s, 3H), $1.21-1.48(\mathrm{~m}, 4 \mathrm{H}), 1.28(\mathrm{~s}, 6 \mathrm{H}), 1.32(\mathrm{~s}, 6 \mathrm{H}), 3.07$ (d, $J=7.4 \mathrm{~Hz}, 1 \mathrm{H}), 7.38-7.48(\mathrm{~m}, 6 \mathrm{H}), 7.79-7.89$ (m, 4H). ${ }^{13} \mathrm{C} \mathrm{NMR}\left(99 \mathrm{MHz}, \mathrm{CDCl}_{3}, \delta\right): 15.3\left(\mathrm{CH}_{3}\right), 16.8\left(\mathrm{~d}, J=4.8 \mathrm{~Hz} \mathrm{CH}_{3}\right), 17.7\left(\mathrm{CH}_{2}\right), 22.0(\mathrm{~d}, J$ $\left.=28.4 \mathrm{~Hz}, \mathrm{CH}_{3}\right), 25.0\left(\mathrm{CH}_{3}\right), 25.1\left(\mathrm{CH}_{3}\right), 39.3(\mathrm{~d}, J=4.8 \mathrm{~Hz}, C), 39.9\left(\mathrm{CH}_{2}\right), 53.5(\mathrm{br}, \mathrm{B}-\mathrm{C}), 128.3$ $(\mathrm{CH}), 128.4(\mathrm{CH}), 131.19$ (d, $J=2.8 \mathrm{~Hz}, C \mathrm{H}), 131.23$ (d, $J=2.9 \mathrm{~Hz}, \mathrm{CH}), 131.7$ (d, $J=9.4 \mathrm{~Hz}, C \mathrm{H})$, $131.9(\mathrm{~d}, J=9.4 \mathrm{~Hz}, C \mathrm{H}), 135.5(C), 136.8(\mathrm{~d}, J=5.6 \mathrm{~Hz}, C) .{ }^{11} \mathrm{~B}\left\{{ }^{1} \mathrm{H}\right\}$ NMR $\left(127 \mathrm{MHz}, \mathrm{CDCl}_{3}, \delta\right)$ : 33.7. ${ }^{31} \mathrm{P}\left\{{ }^{1} \mathrm{H}\right\}$ NMR $\left(159 \mathrm{MHz}, \mathrm{CDCl}_{3}, \delta\right): 22.5$. HRMS-ESI $(\mathrm{m} / \mathrm{z}):[\mathrm{M}+\mathrm{H}]^{+}$calcd for $\mathrm{C}_{26} \mathrm{H}_{40}{ }^{11} \mathrm{BNO}_{3} \mathrm{P}$, 456.2838; found, 456.2834. $[\alpha]_{\mathrm{D}}{ }^{27.9}+2.15$ ( $c 0.93$ in $\mathrm{CHCl}_{3}, 99 \%$ ee). Daicel CHIRALPAK $® \mathrm{OZ}-3$, 2-PrOH$/$ Hexane $=10 / 90,0.5 \mathrm{~mL} / \mathrm{min}, 40^{\circ} \mathrm{C}, S$ isomer: $t_{S}=9.39 \mathrm{~min} ., R$ isomer: $t_{R}=12.77 \mathrm{~min}$. IR 
(neat, $\left.\mathrm{cm}^{-1}\right): 1199(\mathrm{P}=\mathrm{O})$.

(R)-P,P-Diphenyl- $N$-[1-(1-phenylcyclobutyl)-1-(4,4,5,5-tetramethyl-1,3,2-dioxaborolan-2yl)ethyl)phosphinic amide $[(R)-3 g]$.

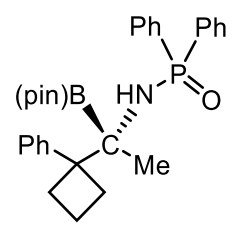

$(R)-3 \mathbf{g}$

The reaction was conducted with $74.2 \mathrm{mg}(0.20 \mathrm{mmol})$ of $\mathbf{1 g}$ for $2 \mathrm{~h}$. The product $(R)-\mathbf{3 g}$ was purified by flash column chromatography $\left(\mathrm{SiO}_{2}\right.$, hexane/EtOAc, 100:0 $\left.\rightarrow 0: 100\right)$ and extracted with $\mathrm{H}_{2} \mathrm{O}$ to remove pinacol, and dried over $\mathrm{MgSO}_{4}$. After filtration, $(R)-\mathbf{3 g}$ was obtained in $74 \%$ yield (73.4 mg, $0.15 \mathrm{mmol}$, colorless oil) with $98 \%$ ee.

${ }^{1} \mathrm{H}$ NMR (396 MHz, $\mathrm{CDCl}_{3}, \delta$ ): 1.09 (s, 3H), 1.30 (s, 6H), 1.34 (s, 6H), 1.75-1.89 (m, 2H), 2.37$2.44(\mathrm{~m}, 2 \mathrm{H}), 2.67-2.74(\mathrm{~m}, 1 \mathrm{H}), 2.77-2.84(\mathrm{~m}, 1 \mathrm{H}), 2.88(\mathrm{~d}, J=5.5 \mathrm{~Hz}, 1 \mathrm{H}), 7.20-7.48(\mathrm{~m}, 13 \mathrm{H})$, 7.78-7.83 (m, 2H). ${ }^{13} \mathrm{C}$ NMR (99 MHz, $\left.\mathrm{CDCl}_{3}, \delta\right): 15.5\left(\mathrm{CH}_{2}\right), 17.7\left(\mathrm{~d}, J=3.8 \mathrm{~Hz}, \mathrm{CH}_{3}\right), 25.3\left(\mathrm{CH}_{3}\right)$, $30.4\left(\mathrm{~d}, J=31.1 \mathrm{~Hz}, C_{2}\right), 51.8(\mathrm{br}, \mathrm{B}-C), 52.6(\mathrm{~d}, J=6.5 \mathrm{~Hz}, C), 84.3(\mathrm{C}), 125.9(\mathrm{CH}), 127.3(\mathrm{CH})$, $128.1(\mathrm{~d}, J=12.3 \mathrm{~Hz}, C \mathrm{H}), 128.3(\mathrm{~d}, J=13.3 \mathrm{~Hz}, C \mathrm{H}), 128.9(C \mathrm{H}), 131.1(\mathrm{~d}, J=1.9 \mathrm{~Hz}, C \mathrm{H}), 131.2$ (d, $J=2.8 \mathrm{~Hz}, C \mathrm{H}), 131.7$ (d, $J=9.5 \mathrm{~Hz}, C \mathrm{H}), 135.3(\mathrm{~d}, J=34.0 \mathrm{~Hz}, C), 136.6(\mathrm{~d}, J=28.3 \mathrm{~Hz}, C)$, $147.1(C) .{ }^{11} \mathrm{~B}\left\{{ }^{1} \mathrm{H}\right\}$ NMR $\left(127 \mathrm{MHz}, \mathrm{CDCl}_{3}, \delta\right): 32.7 .{ }^{31} \mathrm{P}\left\{{ }^{1} \mathrm{H}\right\}$ NMR $\left(159 \mathrm{MHz}, \mathrm{CDCl}_{3}, \delta\right): 22.4$. HRMS-ESI $(\mathrm{m} / \mathrm{z})$ : $[\mathrm{M}+\mathrm{Na}]^{+}$calcd for $\mathrm{C}_{30} \mathrm{H}_{37}{ }^{11} \mathrm{BNO}_{3} \mathrm{PNa}, 524.2502$; found, 524.2501. $[\alpha]_{\mathrm{D}}{ }^{28.0}+1.67$ (c 1.50 in $\mathrm{CHCl}_{3}, 98 \%$ ee). Daicel CHIRALPAK ${ }^{\circledR} \mathrm{OZ}-3,2-\mathrm{PrOH} / \mathrm{Hexane}=10 / 90,0.5 \mathrm{~mL} / \mathrm{min}, 40^{\circ} \mathrm{C}$, $S$ isomer: $t_{S}=10.85$ min., $R$ isomer: $t_{R}=15.55 \min$. $\mathrm{IR}$ (neat, $\left.\mathrm{cm}^{-1}\right): 1200(\mathrm{P}=\mathrm{O})$.

(R)-P,P-Diphenyl- $N$-[1-(1-phenylcyclohexyl)-1-(4,4,5,5-tetramethyl-1,3,2-dioxaborolan-2yl)ethyl]phosphinic amide $[(R)-3 h]$.

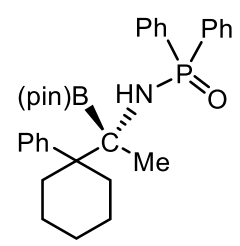

(R)-3h

The reaction was conducted with $79.4 \mathrm{mg}(0.20 \mathrm{mmol})$ of $\mathbf{1 h}$ for $3 \mathrm{~h}$. The product $(R)-\mathbf{3 h}$ was purified by flash column chromatography $\left(\mathrm{SiO}_{2}, \mathrm{CH}_{2} \mathrm{Cl}_{2} / \mathrm{EtOAc}, 100: 0 \rightarrow 90: 10\right.$ to $\mathrm{SiO}_{2}$, EtOAc 
$/ \mathrm{MeOH}, 100: 0 \rightarrow 50: 50$ ) and extracted with $\mathrm{H}_{2} \mathrm{O}$ to remove pinacol, and dried over $\mathrm{MgSO}_{4}$. . After filtration, $(R)-\mathbf{3 h}$ was obtained in $72 \%$ yield $(75.3 \mathrm{mg}, 0.14 \mathrm{mmol}$, white solid) with $97 \%$ ee.

${ }^{1} \mathrm{H}$ NMR $\left(392 \mathrm{MHz}, \mathrm{CDCl}_{3}, \delta\right): 1.06$ (s, 3H), 1.13-1.26 (m, 3H), 1.30 (s, 6H), 1.33 (s, 6H), 1.50 $1.60(\mathrm{~m}, 4 \mathrm{H}), 1.73$ (t, $J=13.1 \mathrm{~Hz}, 1 \mathrm{H}), 2.55$ (t, $J=13.3 \mathrm{~Hz}, 2 \mathrm{H}), 2.93$ (d, $J=3.9 \mathrm{~Hz}, 1 \mathrm{H}), 7.19$ (td, $J$ $=3.1,7.6 \mathrm{~Hz}, 2 \mathrm{H}), 7.27-7.45(\mathrm{~m}, 11 \mathrm{H}), 7.77(\mathrm{ddd}, J=1.5,7.8,11.8 \mathrm{~Hz}, 2 \mathrm{H}) .{ }^{13} \mathrm{C}$ NMR $(100 \mathrm{MHz}$, $\left.\mathrm{CDCl}_{3}, \delta\right): 18.0\left(\mathrm{~d}, J=3.9 \mathrm{~Hz}, \mathrm{CH}_{3}\right), 22.5\left(\mathrm{~d}, J=2.8 \mathrm{~Hz}, \mathrm{CH}_{2}\right), 25.0\left(\mathrm{CH}_{3}\right), 25.2\left(\mathrm{CH}_{3}\right), 26.9\left(\mathrm{CH}_{2}\right)$, $31.5\left(\mathrm{~d}, J=70.9 \mathrm{~Hz}, C \mathrm{H}_{2}\right), 48.8(\mathrm{~d}, J=6.7 \mathrm{~Hz}, C), 53.8$ (br, B-C), $84.3(\mathrm{C}), 125.8(\mathrm{CH}), 127.8(\mathrm{CH})$, $128.0(\mathrm{~d}, J=13.4 \mathrm{~Hz}, C \mathrm{H}), 128.3(\mathrm{~d}, J=12.5 \mathrm{~Hz}, C \mathrm{H}), 130.0(C \mathrm{H}), 130.9(\mathrm{~d}, J=1.9 \mathrm{~Hz}, C \mathrm{H}), 131.1$ (d, $J=1.9 \mathrm{~Hz}, C \mathrm{H}), 131.5$ (d, $J=9.6 \mathrm{~Hz}, C \mathrm{H}), 131.7$ (d, $J=9.6 \mathrm{~Hz}, C \mathrm{H}), 135.5$ (d, $J=38.3 \mathrm{~Hz}, C$ ), $136.7(\mathrm{~d}, J=32.6 \mathrm{~Hz}, C), 140.7(C) .{ }^{11} \mathrm{~B}\left\{{ }^{1} \mathrm{H}\right\} \mathrm{NMR}\left(127 \mathrm{MHz}, \mathrm{CDCl}_{3}, \delta\right): 32.9 .{ }^{31} \mathrm{P}\left\{{ }^{1} \mathrm{H}\right\} \mathrm{NMR}(159$ $\left.\mathrm{MHz}, \mathrm{CDCl}_{3}, \delta\right)$ : 22.7. HRMS-ESI $(m / z)$ : $[\mathrm{M}+\mathrm{H}]^{+}$calcd for $\mathrm{C}_{32} \mathrm{H}_{42}{ }^{11} \mathrm{BNO}_{3} \mathrm{P}, 530.2996$; found, 530.2998. $[\alpha]_{\mathrm{D}} 27.9+0.48\left(c 1.05\right.$ in $\mathrm{CHCl}_{3}, 97 \%$ ee $)$. Daicel CHIRALPAK ${ }^{\circledR} \mathrm{OZ}-3,2-\mathrm{PrOH} / \mathrm{Hexane}=$ 10/90, $0.5 \mathrm{~mL} / \mathrm{min}, 40{ }^{\circ} \mathrm{C}, S$ isomer: $t_{S}=9.92 \mathrm{~min}$., $R$ isomer: $t_{R}=12.13 \mathrm{~min} . \mathrm{mp} 79-81{ }^{\circ} \mathrm{C}$. IR (neat, $\left.\mathrm{cm}^{-1}\right): 1196(\mathrm{P}=\mathrm{O})$.

\section{$N$-\{(R)-1-[(3R,5R,7R)-Adamantan-1-yl]-1-(4,4,5,5-tetramethyl-1,3,2-dioxaborolan-2-yl)ethyl $\}-$}

\section{$\boldsymbol{P}, \boldsymbol{P}$-diphenylphosphinic amide $[(\boldsymbol{R})-3 \mathrm{i}]$.}

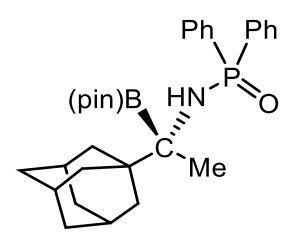

$(R)-3 \mathbf{i}$

The reaction was conducted with $74.2 \mathrm{mg}(0.20 \mathrm{mmol})$ of $1 \mathbf{i}$ for $5 \mathrm{~h}$. The product $(R)-3 \mathbf{i}$ was purified by flash column chromatography $\left(\mathrm{SiO}_{2}\right.$, hexane/EtOAc, 100:0 $\left.\rightarrow 20: 80\right)$ and extracted with $\mathrm{H}_{2} \mathrm{O}$ to remove pinacol, and dried over $\mathrm{MgSO}_{4}$. After filtration, $(R)-3 \mathbf{i}$ was obtained in $87 \%$ yield $(86.8 \mathrm{mg}$, $0.17 \mathrm{mmol}$, white solid) with $99 \%$ ee.

${ }^{1} \mathrm{H}$ NMR $\left(396 \mathrm{MHz}, \mathrm{CDCl}_{3}, \delta\right): 1.14$ (s, 3H), 1.30 (s, 6H), 1.33 (s, 6H), 1.60-1.77 (m, 12H), 2.02 (s, 3H), $3.05(\mathrm{~d}, J=7.9 \mathrm{~Hz}, 1 \mathrm{H}), 7.38-7.48(\mathrm{~m}, 6 \mathrm{H}), 7.80-7.88(\mathrm{~m}, 4 \mathrm{H}) .{ }^{13} \mathrm{C} \mathrm{NMR}\left(100 \mathrm{MHz}, \mathrm{CDCl}_{3}\right.$,

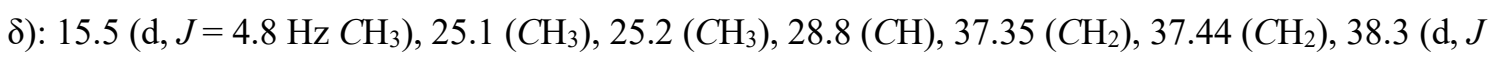
$=4.8 \mathrm{~Hz}, C), 53.0(\mathrm{br}, \mathrm{B}-C), 84.3(C), 128.3(\mathrm{~d}, J=1.9 \mathrm{~Hz}, C \mathrm{H}), 128.4(\mathrm{~d}, J=1.9 \mathrm{~Hz}, C \mathrm{H}), 131.16$ (d, $J=2.9 \mathrm{~Hz}, C \mathrm{H}), 131.23$ (d, $J=1.9 \mathrm{~Hz}, C \mathrm{H}), 131.7$ (d, $J=9.6 \mathrm{~Hz}, C \mathrm{H}), 132.0$ (d, $J=9.6 \mathrm{~Hz}, C \mathrm{H})$, $135.7(\mathrm{~d}, J=3.8 \mathrm{~Hz}, C \mathrm{H}), 137.0(C) .{ }^{11} \mathrm{~B}\left\{{ }^{1} \mathrm{H}\right\} \mathrm{NMR}\left(127 \mathrm{MHz}, \mathrm{CDCl}_{3}, \delta\right): 33.7 .{ }^{31} \mathrm{P}\left\{{ }^{1} \mathrm{H}\right\} \operatorname{NMR}(159$ $\mathrm{MHz}, \mathrm{CDCl}_{3}, \delta$ ): 22.5. HRMS-ESI $(m / z)$ : $[\mathrm{M}+\mathrm{H}]^{+}$calcd for $\mathrm{C}_{30} \mathrm{H}_{42}{ }^{11} \mathrm{BNO}_{3} \mathrm{P}, 506.2995$; found, 
506.2989. $[\alpha]_{\mathrm{D}}^{27.8}-1.34\left(\right.$ c 1.12 in $\mathrm{CHCl}_{3}, 99 \%$ ee $)$. Daicel CHIRALPAK ${ }^{\circledR}$ OZ-3, 2-PrOH/Hexane $=$ $10 / 90,0.5 \mathrm{~mL} / \mathrm{min}, 40{ }^{\circ} \mathrm{C}, S$ isomer: $t_{S}=11.28 \mathrm{~min} ., 2$ isomer: $t_{R}=13.47 \mathrm{~min} . \mathrm{mp} 194-197{ }^{\circ} \mathrm{C}$. IR (neat, $\left.\mathrm{cm}^{-1}\right): 1202(\mathrm{P}=\mathrm{O})$.

\section{(R)-N-[1-Cyclopropyl-1-(4,4,5,5-tetramethyl-1,3,2-dioxaborolan-2-yl)ethyl]-P,P-}

\section{diphenylphosphinic amide $[(R)-3 j]$.}

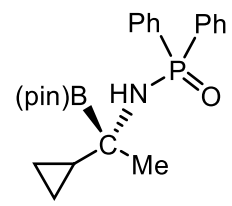

$(R)-\mathbf{3}$

The reaction was conducted with $56.3 \mathrm{mg}(0.20 \mathrm{mmol})$ of $\mathbf{1} \mathbf{j}$ for $2 \mathrm{~h}$. The product $(R)-3 \mathbf{j}$ was purified by flash column chromatography $\left(\mathrm{SiO}_{2}\right.$, hexane/EtOAc, 100:0 $\left.\rightarrow 20: 80\right)$ and extracted with $\mathrm{H}_{2} \mathrm{O}$ to remove pinacol, and dried over $\mathrm{MgSO}_{4}$. After filtration, $(R)-3 \mathbf{j}$ was obtained in $50 \%$ yield $(40.5 \mathrm{mg}$, $0.10 \mathrm{mmol}$, colorless oil) with $86 \%$ ee.

${ }^{1} \mathrm{H}$ NMR (392 MHz, $\left.\mathrm{CDCl}_{3}, \delta\right): 0.29-0.32(\mathrm{~m}, 2 \mathrm{H}), 0.34-0.46$ (m, 2H), 1.04-1.14 (m, 1H), 1.24 (s, $3 \mathrm{H}), 1.25(\mathrm{~s}, 6 \mathrm{H}), 1.26(\mathrm{~s}, 6 \mathrm{H}), 3.08(\mathrm{~d}, J=8.6 \mathrm{~Hz}, 1 \mathrm{H}), 7.38-7.48(\mathrm{~m}, 6 \mathrm{H}), 7.82-7.90(\mathrm{~m}, 4 \mathrm{H}) .{ }^{13} \mathrm{C}$ NMR (99 MHz, $\left.\mathrm{CDCl}_{3}, \delta\right): 1.93\left(\mathrm{CH}_{2}\right), 2.56\left(\mathrm{CH}_{2}\right), 21.6(\mathrm{~d}, J=5.6 \mathrm{~Hz}, \mathrm{CH}), 22.9(\mathrm{~d}, J=3.8 \mathrm{~Hz}$, $\left.\mathrm{CH}_{3}\right), 24.8\left(\mathrm{CH}_{3}\right), 24.9\left(\mathrm{CH}_{3}\right), 84.3(\mathrm{C}), 128.2(\mathrm{~d}, J=2.9 \mathrm{~Hz}, \mathrm{CH}), 128.4(\mathrm{~d}, J=3.8 \mathrm{~Hz}, \mathrm{CH}), 131.29$ $(C H), 131.31(C H), 131.9(\mathrm{~d}, J=9.6 \mathrm{~Hz}, C \mathrm{H}), 132.0(\mathrm{~d}, J=8.5 \mathrm{~Hz}, C \mathrm{H}), 135.1(C), 136.4(C)$. The carbon directly attached to the boron atom was not detected, likely because of quadrupolar relaxation. ${ }^{11} \mathrm{~B}\left\{{ }^{1} \mathrm{H}\right\}$ NMR (127 MHz, $\left.\mathrm{CDCl}_{3}, \delta\right): 33.0 .{ }^{31} \mathrm{P}\left\{{ }^{1} \mathrm{H}\right\}$ NMR (159 MHz, $\left.\mathrm{CDCl}_{3}, \delta\right): 22.2$. HRMS-ESI $(m / z):[\mathrm{M}+\mathrm{Na}]^{+}$calcd for $\mathrm{C}_{23} \mathrm{H}_{31}{ }^{11} \mathrm{BNO}_{3} \mathrm{PNa}, 434.2031$; found, 434.2029. [ $\left.\alpha\right]_{\mathrm{D}}{ }^{25.2}-2.44$ (c 1.17 in $\mathrm{CHCl}_{3}, 86 \%$ ee). Daicel CHIRALPAK ${ }^{\circledR} \mathrm{OZ}-3,2-\mathrm{PrOH} / \mathrm{Hexane}=10 / 90,0.5 \mathrm{~mL} / \mathrm{min}, 40{ }^{\circ} \mathrm{C}, S$ isomer: $t_{S}=21.95 \mathrm{~min} ., R$ isomer: $t_{R}=28.56 \mathrm{~min} . \mathrm{IR}$ (neat, $\left.\mathrm{cm}^{-1}\right): 1197(\mathrm{P}=\mathrm{O})$. 
(R)- $N$-[2,2-Dimethyl-3-(4,4,5,5-tetramethyl-1,3,2-dioxaborolan-2-yl)heptan-3-yl]-P,Pdiphenylphosphinic amide $[(R)-3 k]$.

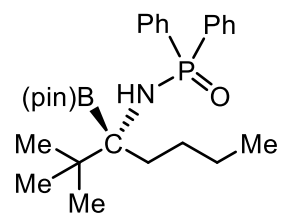

$(R)-3 \mathbf{k}$

The reaction was conducted with $67.2 \mathrm{mg}(0.20 \mathrm{mmol})$ of $\mathbf{1 k}$ for $2 \mathrm{~h}$. The product $(R)-\mathbf{3 k}$ was purified by flash column chromatography $\left(\mathrm{SiO}_{2}\right.$, hexane/EtOAc, 100:0 $\left.\rightarrow 20: 80\right)$ and extracted with $\mathrm{H}_{2} \mathrm{O}$ to remove pinacol, and dried over $\mathrm{MgSO}_{4}$. After filtration, $(R)-\mathbf{3 k}$ was obtained in $80 \%$ yield (73.6 mg, $0.16 \mathrm{mmol}$, colorless oil) with $86 \%$ ee.

${ }^{1} \mathrm{H}$ NMR (392 MHz, $\left.\mathrm{CDCl}_{3}, \delta\right): 0.63(\mathrm{t}, J=7.3 \mathrm{~Hz}, 3 \mathrm{H}), 0.84-1.21(\mathrm{~m}, 4 \mathrm{H}), 1.03(\mathrm{~s}, 9 \mathrm{H}), 1.25$ (s, $6 \mathrm{H}), 1.26(\mathrm{~s}, 6 \mathrm{H}), 1.62(\mathrm{dt}, J=6.5,18.8 \mathrm{~Hz}, 1 \mathrm{H}), 1.82-1.94(\mathrm{~m}, 1 \mathrm{H}), 3.04(\mathrm{~d}, J=11.0 \mathrm{~Hz}, 1 \mathrm{H}), 7.37-$ $7.46(\mathrm{~m}, 6 \mathrm{H}), 7.73-7.79(\mathrm{~m}, 2 \mathrm{H}), 7.86-7.91(\mathrm{~m}, 2 \mathrm{H}) .{ }^{13} \mathrm{C} \mathrm{NMR}\left(99 \mathrm{MHz}, \mathrm{CDCl}_{3}, \delta\right): 14.1\left(\mathrm{CH}_{3}\right), 23.5$ $\left(\mathrm{CH}_{2}\right), 25.0\left(\mathrm{CH}_{3}\right), 25.2\left(\mathrm{CH}_{3}\right), 27.3\left(\mathrm{CH}_{3}\right), 29.7\left(\mathrm{CH}_{2}\right), 31.3\left(\mathrm{~d}, J=2.8 \mathrm{~Hz}, \mathrm{CH}_{2}\right), 37.9(\mathrm{C}), 60.0$ (br, B-C), $84.4(C), 128.0(\mathrm{~d}, J=13.2 \mathrm{~Hz}, C \mathrm{H}), 128.3$ (d, $J=12.3 \mathrm{~Hz}, C \mathrm{H}), 130.9$ (d, $J=2.9 \mathrm{~Hz}, C \mathrm{H})$, 131.0 (d, $J=1.9 \mathrm{~Hz}, C \mathrm{H}), 131.3$ (d, $J=10.4 \mathrm{~Hz}, C \mathrm{H}), 132.2$ (d, $J=10.4 \mathrm{~Hz}, C \mathrm{H}), 136.6$ (d, $J=61.4$ $\mathrm{Hz}, C), 137.9$ (d, $J=59.5 \mathrm{~Hz}, C) .{ }^{11} \mathrm{~B}\left\{{ }^{1} \mathrm{H}\right\}$ NMR $\left(127 \mathrm{MHz}, \mathrm{CDCl}_{3}, \delta\right): 33.0 .{ }^{31} \mathrm{P}\left\{{ }^{1} \mathrm{H}\right\} \mathrm{NMR}(159$ $\left.\mathrm{MHz}, \mathrm{CDCl}_{3}, \delta\right): 22.2$. HRMS-ESI $(\mathrm{m} / \mathrm{z})$ : $[\mathrm{M}+\mathrm{H}]^{+}$calcd for $\mathrm{C}_{27} \mathrm{H}_{42}{ }^{11} \mathrm{BNO}_{3} \mathrm{P}, 470.2995$; found, 470.2989. $[\alpha]_{\mathrm{D}}^{25.7}+1.40$ (c 3.67 in $\mathrm{CHCl}_{3}, 88 \%$ ee $)$. Daicel CHIRALPAK $®$ OZ-3, 2-PrOH/Hexane $=$ 10/90, $0.5 \mathrm{~mL} / \mathrm{min}, 40^{\circ} \mathrm{C}, S$ isomer: $t_{S}=9.49 \min ., R$ isomer: $t_{R}=12.08 \mathrm{~min}$. IR (neat, $\mathrm{cm}^{-1}$ ): 1204 $(\mathrm{P}=\mathrm{O})$.

(R)- $N$-[4-Methyl-2-(4,4,5,5-tetramethyl-1,3,2-dioxaborolan-2-yl)pentan-2-yl]-P,Pdiphenylphosphinic amide $[(R)-31]$.

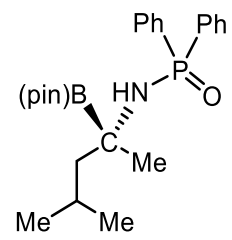

$(R)-31$

The reaction was conducted with $60.2 \mathrm{mg}(0.20 \mathrm{mmol})$ of $1 \mathbf{i}$ for $2 \mathrm{~h}$. The product $(R)-3 \mathbf{i}$ was purified by flash column chromatography $\left(\mathrm{SiO}_{2}\right.$, hexane/EtOAc, 100:0 $\left.\rightarrow 40: 60\right)$ and extracted with $\mathrm{H}_{2} \mathrm{O}$ to remove pinacol, and dried over $\mathrm{MgSO}_{4}$. After filtration, $(R)-31$ was obtained in $86 \%$ yield $(73.5 \mathrm{mg}$, $0.17 \mathrm{mmol}$, colorless oil) with $88 \%$ ee. 
${ }^{1} \mathrm{H}$ NMR (392 MHz, $\left.\mathrm{CDCl}_{3}, \delta\right): 0.90$ (d, $\left.J=6.7, \mathrm{~Hz}, 3 \mathrm{H}\right), 0.93(\mathrm{~d}, J=6.7, \mathrm{~Hz}, 3 \mathrm{H}), 1.23$ (s, 3H), $1.26(\mathrm{~s}, 6 \mathrm{H}), 1.28(\mathrm{~s}, 6 \mathrm{H}), 1.44(\mathrm{dd}, J=7.6,13.9 \mathrm{~Hz}, 1 \mathrm{H}), 1.68(\mathrm{dd}, J=5.5,13.3 \mathrm{~Hz}, 1 \mathrm{H}), 1.83-1.97$ (m, 1H), $3.11(\mathrm{~d}, J=9.0 \mathrm{~Hz}, 1 \mathrm{H}), 7.39-7.50(\mathrm{~m}, 6 \mathrm{H}), 7.77-7.83(\mathrm{~m}, 2 \mathrm{H}), 7.87-7.93(\mathrm{~m}, 2 \mathrm{H}) .{ }^{13} \mathrm{C} \mathrm{NMR}$ (99 MHz, $\left.\mathrm{CDCl}_{3}, \delta\right): 23.7\left(\mathrm{CH}_{3}\right), 24.2\left(\mathrm{CH}_{3}\right), 24.6\left(\mathrm{~d}, J=4.8 \mathrm{~Hz}, \mathrm{CH}_{3}\right), 25.0\left(\mathrm{CH}_{3}\right), 25.3(\mathrm{CH}), 45.7$ (br, B-C), 50.6 (d, $\left.J=2.9 \mathrm{~Hz}, C \mathrm{H}_{2}\right), 84.4(C), 128.3$ (d, $\left.J=3.8 \mathrm{~Hz}, C \mathrm{H}\right), 128.4$ (d, $J=3.8 \mathrm{~Hz}, C \mathrm{H}$ ), $131.3(C \mathrm{H}), 131.7(\mathrm{~d}, J=9.5 \mathrm{~Hz}, C \mathrm{H}), 132.1(\mathrm{~d}, J=9.4 \mathrm{~Hz}, C \mathrm{H}), 135.2(C), 136.5(C) .{ }^{11} \mathrm{~B}\left\{{ }^{1} \mathrm{H}\right\} \mathrm{NMR}$ $\left(127 \mathrm{MHz}, \mathrm{CDCl}_{3}, \delta\right): 33.8 .{ }^{31} \mathrm{P}\left\{{ }^{1} \mathrm{H}\right\}$ NMR $\left(159 \mathrm{MHz}, \mathrm{CDCl}_{3}, \delta\right): 21.9$. HRMS-ESI $(m / z):[\mathrm{M}+\mathrm{Na}]^{+}$ calcd for $\mathrm{C}_{24} \mathrm{H}_{35}{ }^{11} \mathrm{BNO}_{3} \mathrm{P}, 450.2344$; found, 450.2340. $[\alpha]_{\mathrm{D}}{ }^{25.9}+1.34$ (c 4.28 in $\mathrm{CHCl}_{3}, 88 \%$ ee). Daicel CHIRALPAK ${ }^{\circledR}$ OZ-3, 2-PrOH $/$ Hexane $=10 / 90,0.5 \mathrm{~mL} / \mathrm{min}, 40{ }^{\circ} \mathrm{C}, S$ isomer: $t_{S}=13.73 \mathrm{~min} ., R$ isomer: $t_{R}=17.52 \mathrm{~min}$. IR (neat, $\left.\mathrm{cm}^{-1}\right): 1198(\mathrm{P}=\mathrm{O})$.

\section{(R)-P,P-Diphenyl- $N$-[4-phenyl-2-(4,4,5,5-tetramethyl-1,3,2-dioxaborolan-2-yl)butan-2-}

\section{yl]phosphinic amide $[(R)-3 m]$.}

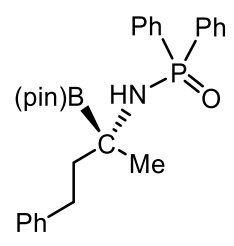

(R)-3m

The reaction was conducted with $69.1 \mathrm{mg}(0.20 \mathrm{mmol})$ of $\mathbf{1 m}$ for $6 \mathrm{~h}$. The product $(R)-\mathbf{3 m}$ was purified by flash column chromatography $\left(\mathrm{SiO}_{2}\right.$, hexane/EtOAc, 100:0 $\left.\rightarrow 40: 60\right)$ and extracted with $\mathrm{H}_{2} \mathrm{O}$ to remove pinacol, and dried over $\mathrm{MgSO}_{4}$. After filtration, $(R)-3 \mathbf{m}$ was obtained in $44 \%$ yield (41.4 mg, $0.09 \mathrm{mmol}$, colorless oil) with $89 \%$ ee.

${ }^{1} \mathrm{H}$ NMR $\left(392 \mathrm{MHz}, \mathrm{CDCl}_{3}, \delta\right): 1.285-1.294(\mathrm{~m}, 15 \mathrm{H}), 1.82(\mathrm{td}, J=4.3,12.9 \mathrm{~Hz}, 1 \mathrm{H}), 2.00(\mathrm{td}, J=$ $5.0,13.0 \mathrm{~Hz}, 1 \mathrm{H}), 2.57(\mathrm{td}, J=4.7,12.9 \mathrm{~Hz}, 1 \mathrm{H}), 2.87(\mathrm{td}, J=4.8,12.9 \mathrm{~Hz}, 1 \mathrm{H}), 3.21(\mathrm{~d}, J=8.6 \mathrm{~Hz}$, 1H), 7.13-7.16 (m, 3H), 7.23-7.27 (m, 2H), 7.39-7.52 (m, 6H) , 7.83-7.92 (m, 4H). ${ }^{13} \mathrm{C}$ NMR (99 $\left.\mathrm{MHz}, \mathrm{CDCl}_{3}, \delta\right): 24.2\left(\mathrm{~d}, J=3.8 \mathrm{~Hz}, \mathrm{CH}_{3}\right), 24.9\left(\mathrm{CH}_{3}\right), 25.0\left(\mathrm{CH}_{3}\right), 32.3\left(\mathrm{CH}_{2}\right), 43.5(\mathrm{~d}, J=3.8 \mathrm{~Hz}$, $\mathrm{CH}_{2}$ ), 46.1 (br, B-C), $84.5(\mathrm{C}), 125.8(\mathrm{CH}), 128.3$ (d, $\left.J=2.9 \mathrm{~Hz}, \mathrm{CH}\right), 128.40(\mathrm{CH}), 128.45$ (d, $J=1.9$ $\mathrm{Hz}, C \mathrm{H}), 128.55(\mathrm{CH}), 135.0(\mathrm{~d}, J=5.7 \mathrm{~Hz}, C), 136.3(\mathrm{~d}, J=6.6 \mathrm{~Hz}, C), 142.7(C) .{ }^{11} \mathrm{~B}\left\{{ }^{1} \mathrm{H}\right\} \mathrm{NMR}$ $\left(127 \mathrm{MHz}, \mathrm{CDCl}_{3}, \delta\right): 32.6 .{ }^{31} \mathrm{P}\left\{{ }^{1} \mathrm{H}\right\}$ NMR $\left(159 \mathrm{MHz}, \mathrm{CDCl}_{3}, \delta\right): 22.1$. HRMS-ESI $(m / z):[\mathrm{M}+\mathrm{Na}]^{+}$ calcd for $\mathrm{C}_{28} \mathrm{H}_{35}{ }^{11} \mathrm{BNO}_{3} \mathrm{PNa}, 498.2345$; found, 498.2339. [ $\left.\alpha\right]_{\mathrm{D}}{ }^{22.0}-13.9$ (c 0.85 in $\mathrm{CHCl}_{3}, 89 \%$ ee). Daicel CHIRALPAK ${ }^{\circledR}$ OZ-3, 2-PrOH/Hexane $=5 / 95,0.5 \mathrm{~mL} / \mathrm{min}, 40{ }^{\circ} \mathrm{C}, S$ isomer: $t_{S}=32.80 \mathrm{~min}$, $R$ isomer: $t_{R}=47.56 \mathrm{~min}$. IR (neat, $\left.\mathrm{cm}^{-1}\right): 1196(\mathrm{P}=\mathrm{O})$. 
(R)-P,P-Diphenyl- $N$-[2-(4,4,5,5-tetramethyl-1,3,2-dioxaborolan-2-yl)butan-2-yl]phosphinic amide $[(R)-3 n]$.

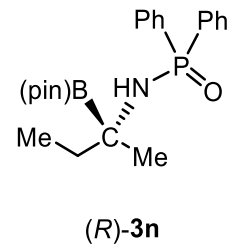

The reaction was conducted with $53.8 \mathrm{mg}(0.20 \mathrm{mmol})$ of $\mathbf{1 n}$ for $1 \mathrm{~h}$. The product $(R)-\mathbf{3 n}$ was purified by flash column chromatography $\left(\mathrm{SiO}_{2}\right.$, hexane/EtOAc, 100:0 $\left.\rightarrow 50: 50\right)$ and extracted with $\mathrm{H}_{2} \mathrm{O}$ to remove pinacol, and dried over $\mathrm{MgSO}_{4}$. After filtration, $(R)-3 \mathbf{n}$ was obtained in $28 \%$ yield (22.3 mg, $0.06 \mathrm{mmol}$, colorless oil) with $69 \%$ ee.

${ }^{1} \mathrm{H}$ NMR (392 MHz, $\left.\mathrm{CDCl}_{3}, \delta\right): 0.94(\mathrm{t}, J=7.4 \mathrm{~Hz}, 3 \mathrm{H}), 1.23(\mathrm{~s}, 3 \mathrm{H}), 1.26(\mathrm{~s}, 6 \mathrm{H}), 1.27(\mathrm{~s}, 6 \mathrm{H})$, 1.57 (sxt, $J=7.2 \mathrm{~Hz}, 1 \mathrm{H}), 1.70$ (sxt, $J=7.1 \mathrm{~Hz}, 1 \mathrm{H}), 3.11$ (d, $J=9.0 \mathrm{~Hz}, 1 \mathrm{H}), 7.39-7.49$ (m, 6H), 7.82-7.91 (m, 4H). ${ }^{13} \mathrm{C}$ NMR (99 MHz, $\left.\mathrm{CDCl}_{3}, \delta\right): 10.1\left(\mathrm{CH}_{3}\right), 23.6\left(\mathrm{~d}, J=3.8 \mathrm{~Hz}, \mathrm{CH}_{3}\right), 24.9\left(\mathrm{CH}_{3}\right)$, $25.0\left(\mathrm{CH}_{3}\right), 34.2\left(\mathrm{~d}, J=3.8 \mathrm{~Hz}, \mathrm{CH}_{2}\right), 46.7(\mathrm{br}, \mathrm{B}-\mathrm{C}), 84.3(\mathrm{C}), 128.2(\mathrm{CH}), 128.4(\mathrm{CH}), 131.3(\mathrm{CH})$, $131.8(\mathrm{~d}, J=10.4 \mathrm{~Hz}, C \mathrm{H}), 132.1(\mathrm{~d}, J=10.4 \mathrm{~Hz}, C \mathrm{H}), 135.1(C), 136.4(C) .{ }^{11} \mathrm{~B}\left\{{ }^{1} \mathrm{H}\right\} \operatorname{NMR}(127$ $\left.\mathrm{MHz}, \mathrm{CDCl}_{3}, \delta\right): 32.5 .{ }^{31} \mathrm{P}\left\{{ }^{1} \mathrm{H}\right\}$ NMR (162 MHz, $\left.\mathrm{CDCl}_{3}, \delta\right): 22.0$. HRMS-ESI $(\mathrm{m} / \mathrm{z}):[\mathrm{M}+\mathrm{Na}]^{+}$calcd for $\mathrm{C}_{22} \mathrm{H}_{31}{ }^{11} \mathrm{BNO}_{3} \mathrm{P}, 422.2031$; found, 422.2026. $[\alpha]_{\mathrm{D}}{ }^{25.0}+18.9$ (c 0.12 in $\mathrm{CHCl}_{3}, 69 \%$ ee). Daicel CHIRALPAK ${ }^{\circledR}$ OZ-3, 2-PrOH/Hexane $=10 / 90,0.5 \mathrm{~mL} / \mathrm{min}, 40{ }^{\circ} \mathrm{C}, S$ isomer: $t_{S}=19.49 \min ., R$ isomer: $t_{R}=24.40 \mathrm{~min}$. IR (neat, $\left.\mathrm{cm}^{-1}\right): 1196(\mathrm{P}=\mathrm{O})$. 


\section{Experimental Procedures for the Synthesis of Peptydilboronic Acid Derivatives}

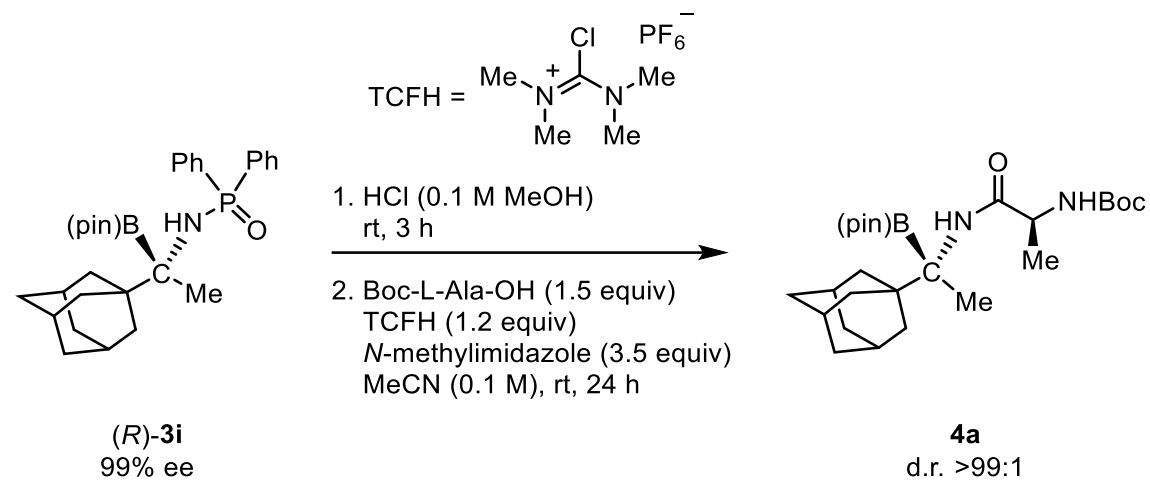

The borylation product $(R)-3 \mathbf{i}(177 \mathrm{mg}, 0.35 \mathrm{mmol}, 1.0$ equiv) was placed in a vial with a screw cap containing a Teflon ${ }^{\circledR}$-coated rubber septum under air. A MeOH solution of $\mathrm{HCl}(0.1 \mathrm{M}, 3.5 \mathrm{~mL})$ was added to the vial via a syringe. The resulting mixture was stirred for $3 \mathrm{~h}$ at room temperature. The solvents are removed under reduced pressure to afford the deprotection product. The product was used in the next step without further purification. The condensation reaction was performed according to the literature. ${ }^{7}$. Boc-L-Ala-OH ( $85.9 \mathrm{mg}, 0.46 \mathrm{mmol}, 1.3$ equiv) and TCFH (118 mg, $0.42 \mathrm{mmol}, 1.2$ equiv) were placed in a vial with a screw cap containing a Teflon ${ }^{\circledR}$-coated rubber septum under air. $\mathrm{MeCN}(100 \mu \mathrm{L})$ was added to the vial via a syringe. The resulting mixture was stirred for $5 \mathrm{~min}$ at room temperature. A THF solution $(250 \mu \mathrm{L})$ of the deprotection product was then added dropwise to the vial. $N$-Metylimidazole ( $94 \mu \mathrm{L}, 1.2 \mathrm{mmol}, 3.5$ equiv) was added dropwise to the vial. The reaction mixture was stirred for $24 \mathrm{~h}$. The reaction mixture was analyzed by TLC to check the completeness of the reaction. The mixture was directly filtered through a short silica-gel column with EtOAc as an eluent, then the resultant solution was concentrated under reduced pressure. The crude product was purified by silica-gel column chromatography with hexane/EtOAc eluent (100:0 to 40:60) to give the corresponding product $4 \mathbf{a}$ in $71 \%$ yield $(118.0 \mathrm{mg}, 0.25 \mathrm{mmol})$ as white solid. The diastereomeric ratio was determined by HPLC analysis (d.r. $>99: 1$ ). 
dioxaborolan-2-yl)ethyl\}amino)-1-oxopropan-2-yl]carbamate (4a).

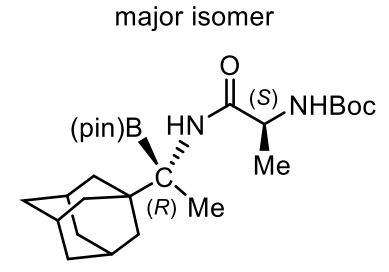

$4 a$

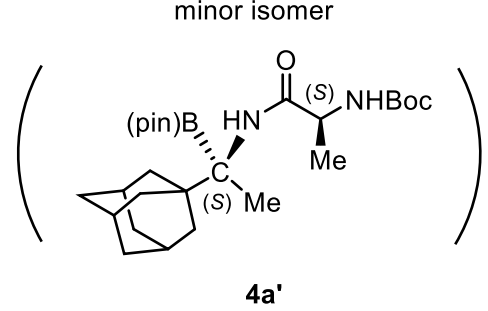

$4 a^{\prime}$

NMR spectra for $4 \mathbf{a}$ contains conformational isomers, which is caused by the restricted $\mathrm{C}-\mathrm{N}$ bond rotation around the carbamate group. ${ }^{1} \mathrm{H}$ NMR $\left(392 \mathrm{MHz}, \mathrm{CDCl}_{3}, \delta\right): 1.14(\mathrm{~s}, 3 \mathrm{H}), 1.23(\mathrm{~s}, 6 \mathrm{H}), 1.24$ (s, 6H), $1.35(\mathrm{~d}, J=7.4 \mathrm{~Hz}, 3 \mathrm{H}), 1.45$ (s, 9H), 1.60-1.76 (m, 12H), $1.96(\mathrm{~s}, 3 \mathrm{H}), 4.09-4.29(\mathrm{~m}, 1 \mathrm{H})$, 4.73-5.01 (m, 1H), 6.59-6.87 (m, 1H). $\left.{ }^{13} \mathrm{C} \mathrm{NMR} \mathrm{(99} \mathrm{MHz,} \mathrm{CDCl}_{3}, \delta\right): 16.4\left(\mathrm{CH}_{3}\right), 24.9\left(\mathrm{CH}_{3}\right), 25.2$ $\left(\mathrm{CH}_{3}\right), 25.5\left(\mathrm{CH}_{3}\right), 28.4\left(\mathrm{CH}_{3}\right), 28.7(\mathrm{CH}), 36.9\left(\mathrm{CH}_{2}\right), 37.2\left(\mathrm{CH}_{2}\right), 37.5(C), 48.0(\mathrm{CH}), 51.1$ (br, BC), 75.1 and 80.5 (a pair of s, C), $81.9(C), 155.8(C), 173.8(C) .{ }^{11} \mathrm{~B}\left\{{ }^{1} \mathrm{H}\right\} \mathrm{NMR}\left(127 \mathrm{MHz}, \mathrm{CDCl}_{3}\right.$, $\delta): ~ 25.5$. HRMS-ESI $(m / z)$ : $[\mathrm{M}+\mathrm{Na}]^{+}$calcd for $\mathrm{C}_{26} \mathrm{H}_{45} \mathrm{~N}_{2} \mathrm{O}_{5}{ }^{11} \mathrm{BNa}, 499.3319$; found, 499.3313. [ $\left.\alpha\right]_{\mathrm{D}}{ }^{22.3}$ -25.3 ( $c 0.39$ in $\mathrm{CHCl}_{3}$ ). The diastereomeric ratio was determined by HPLC analysis (d.r. $>99: 1$, right side in Figure S1) by comparison with a mixture of the diastereomers that was obtained by the reaction of racemic 3i with Boc-L-Ala-OH (d.r. 80:20, left side in Figure S1). Daicel CHIRALPAK® ID-3, 2$\mathrm{PrOH} / \mathrm{Hexane}=3 / 97,0.5 \mathrm{~mL} / \mathrm{min}, 40{ }^{\circ} \mathrm{C}$, minor isomer $=11.88 \mathrm{~min}$., major isomer $=15.07 \mathrm{~min} . \mathrm{mp}$ 82-92 ${ }^{\circ} \mathrm{C}$. IR (neat, $\left.\mathrm{cm}^{-1}\right): 1699(\mathrm{C}=\mathrm{O}$, amide).
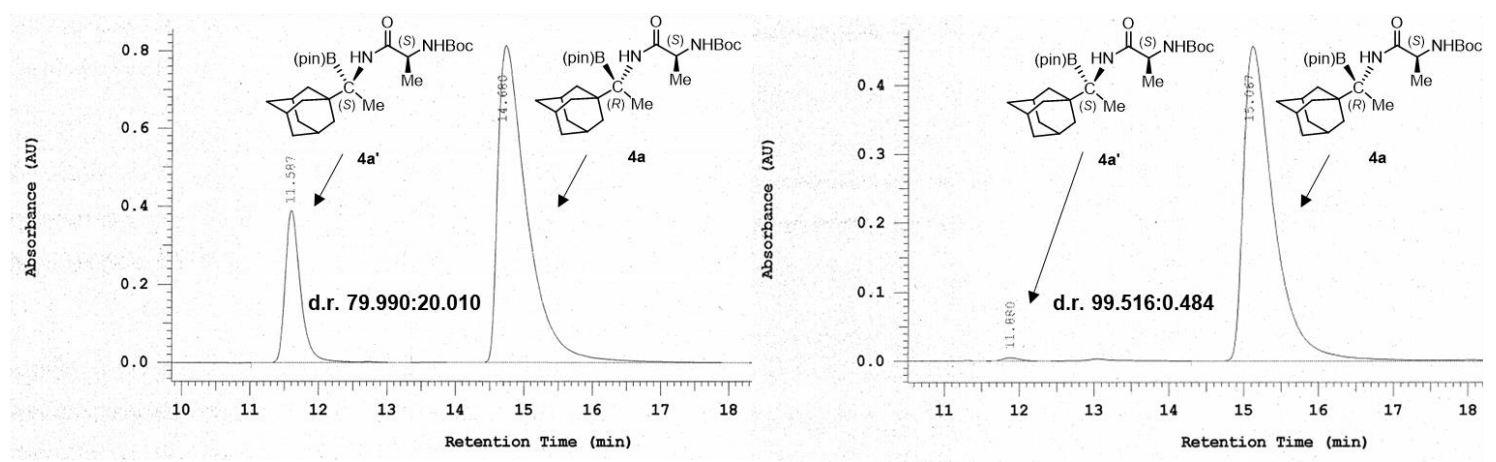

Figure S1. Determination of the diastereomeric ratio of $\mathbf{4 a}$ by HPLC analysis. 
tert-Butyl ((S)-1-oxo-3-phenyl-1-\{[(R)-1-(1-phenylcyclobutyl)-1-(4,4,5,5-tetramethyl-1,3,2dioxaborolan-2-yl)ethyl]amino\}propan-2-yl)carbamate (4b).

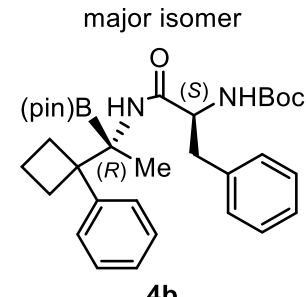

4b

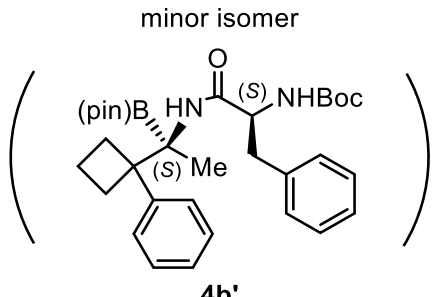

$4 b^{\prime}$

4b was synthesized according to the procedure for the synthesis of $4 \mathbf{a}$. The reaction was conducted with $100.5 \mathrm{mg}(0.20 \mathrm{mmol})$ of $(R)-3 \mathrm{~g}$ using Boc-L-Phe-OH $(79.9 \mathrm{mg}, 0.30 \mathrm{mmol}, 1.5$ equiv) and TCFH (78.2 $\mathrm{mg}, 0.28 \mathrm{mmol}, 1.4$ equiv). The product $\mathbf{4 b}$ was purified by flash column chromatography $\left(\mathrm{SiO}_{2}\right.$, hexane/EtOAc, $\left.100: 0 \rightarrow 70: 30\right)$ to give the corresponding product $\mathbf{4 b}$ in $62 \%$ yield $(69.3 \mathrm{mg}$, $0.12 \mathrm{mmol}$ ) as white solid. The diastereomeric ratio was determined by HPLC analysis (d.r. >99:1).

NMR spectra for $\mathbf{4 b}$ contains conformational isomers, which is caused by the restricted $\mathrm{C}-\mathrm{N}$ bond rotation around the carbamate group. ${ }^{1} \mathrm{H}$ NMR $\left(396 \mathrm{MHz}, \mathrm{CDCl}_{3}, \delta\right.$ ): 0.82 (brs, 3H), 1.29 (s, 6H), 1.35 (s, 6H), 1.35-1.39 (m, 9H), 1.66-1.85 (m, 2H), 2.16-2.23 (m, 1H), 2.34-2.42 (m, 1H), 2.50-2.58 (m, $1 \mathrm{H}), 2.72-2.84(\mathrm{~m}, 1 \mathrm{H}), 2.94(\mathrm{dd}, J=6.9,13.7 \mathrm{~Hz}, 1 \mathrm{H}), 3.13(\mathrm{dd}, J=6.1,14.1 \mathrm{~Hz}, 1 \mathrm{H}), 4.23-4.33$ (m, 1H), 4.88-4.97 (m, 1H), 5.75-5.86 (m, 1H), 7.12-7.31 (m, 10H). $\left.{ }^{13} \mathrm{C} \mathrm{NMR} \mathrm{(100} \mathrm{MHz,} \mathrm{CDCl}_{3}, \delta\right)$ : $15.3\left(\mathrm{CH}_{2}\right), 18.6\left(\mathrm{CH}_{3}\right), 25.4\left(\mathrm{CH}_{3}\right), 25.8\left(\mathrm{CH}_{3}\right), 28.3\left(\mathrm{CH}_{3}\right), 31.0\left(\mathrm{CH}_{2}\right), 38.2\left(\mathrm{CH}_{2}\right), 48.8(\mathrm{br}, \mathrm{B}-\mathrm{C})$, $51.0(C), 54.7(C H), 80.2(C), 82.9(C), 126.0(C H), 127.0(C H), 127.6(C H), 128.6(C H), 128.7(C H)$, 129.6 (CH), $136.4(C), 145.9(C), 155.2(C), 171.8(C) .{ }^{11} \mathrm{~B}\left\{{ }^{1} \mathrm{H}\right\}$ NMR (127 MHz, $\left.\mathrm{CDCl}_{3}, \delta\right): 29.1$. HRMS-ESI $(m / z)$ : $[\mathrm{M}+\mathrm{Na}]^{+}$calcd for $\mathrm{C}_{32} \mathrm{H}_{45} \mathrm{~N}_{2} \mathrm{O}_{5}{ }^{11} \mathrm{BNa}, 571.3319$; found, 571.3316 . [ $\left.\alpha\right]_{\mathrm{D}} 22.0-5.94(c$ 1.10 in $\mathrm{CHCl}_{3}$ ). The diastereomeric ratio was determined by HPLC analysis (d.r. >99:1). Daicel CHIRALPAK $®$ ID-3, 2-PrOH $/$ Hexane $=3 / 97,0.5 \mathrm{~mL} / \mathrm{min}, 40^{\circ} \mathrm{C}$, minor isomer $=8.65 \mathrm{~min}$., major isomer $=12.01 \mathrm{~min} . \mathrm{mp} 62-70{ }^{\circ} \mathrm{C}$. IR (neat, $\left.\mathrm{cm}^{-1}\right): 1708(\mathrm{C}=\mathrm{O}$, amide $)$.
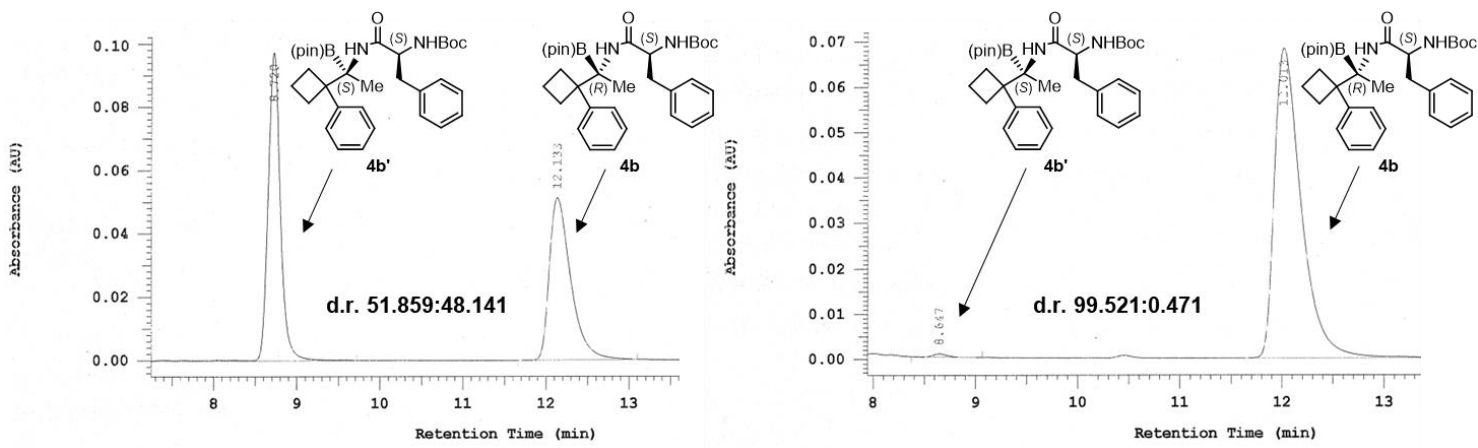

Figure S2. Determination of the diastereomeric ratio of $\mathbf{4 b}$ by HPLC analysis. 


\section{Additional Experimental Results}

Unsuccessful examples of the borylation of ketimines with $\mathrm{P}(\mathrm{O})(\mathrm{o} \text {-tol })_{2}$ as the protecting group

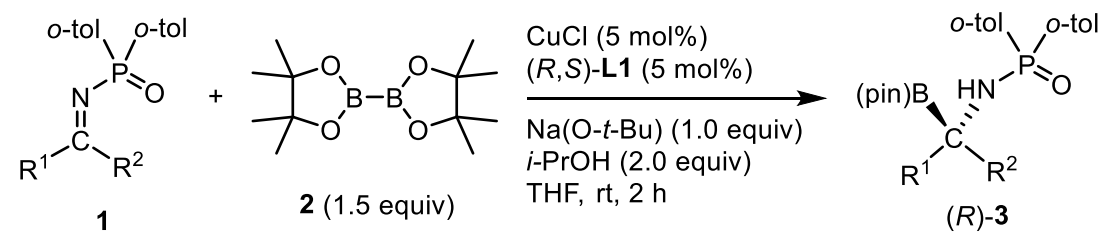

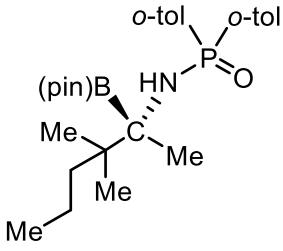

$(R)-3 f^{\prime}$

no reaction

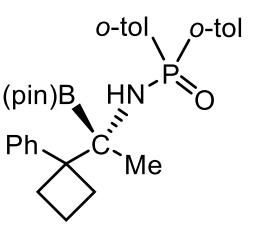

$(R)-3 \mathbf{g}^{\prime}$ no reaction

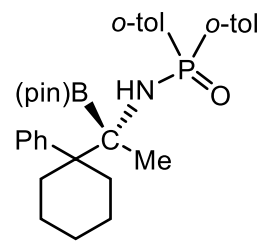

(R)-3h' no reaction

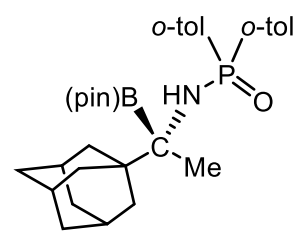

(R)-3i' no reaction

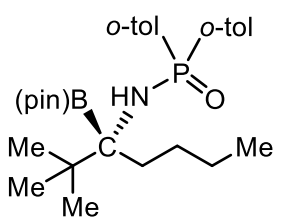

$(R)-3 \mathbf{k}^{\prime}$ no reaction

An unsuccessful example of the borylation of aromatic ketimine 10<smiles>C[N+](=NP(=O)([O-])O[Na])c1ccccc1</smiles>

10<smiles>CC1(C)OB(B2OC(C)(C)C(C)(C)O2)OC1(C)C</smiles>

2 (1.5 equiv)
$\mathrm{CuCl}(5 \mathrm{~mol} \%)$

$(R, S)$-L1 (5 mol\%)

$\mathrm{Na}(\mathrm{O}-\mathrm{t}-\mathrm{Bu})(1.0$ equiv) $i$-PrOH (2.0 equiv) THF, rt, $2 \mathrm{~h}$<smiles>C[C@H](NP(=O)([O-])O[Na])c1ccccc1</smiles>

6

$62 \%$ yield 


\section{Unsuccessful synthesis of the substrate 1n'}
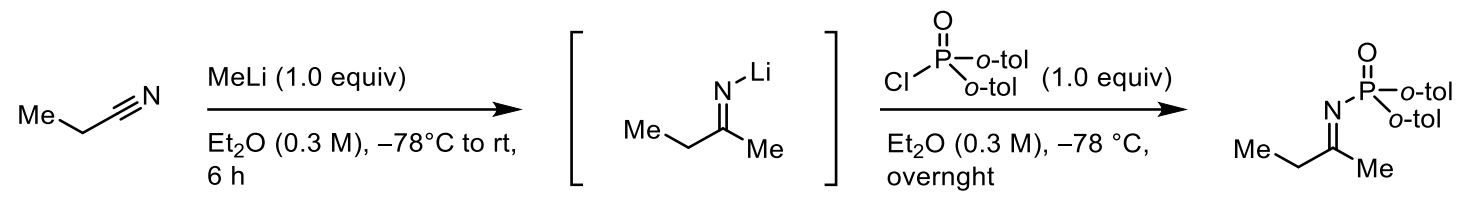

$5.0 \mathrm{mmol}$

$$
\text { overnght }
$$

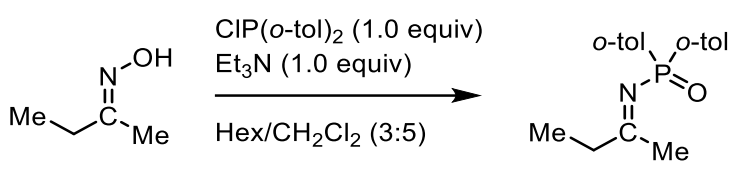

$$
\begin{aligned}
& 6.0 \mathrm{mmol} \\
& \text { 1n' }
\end{aligned}
$$

Neither procedure A nor B could synthesize the ketimine 1n'.

\section{Condensation reactions with Boc-Ala-OH}
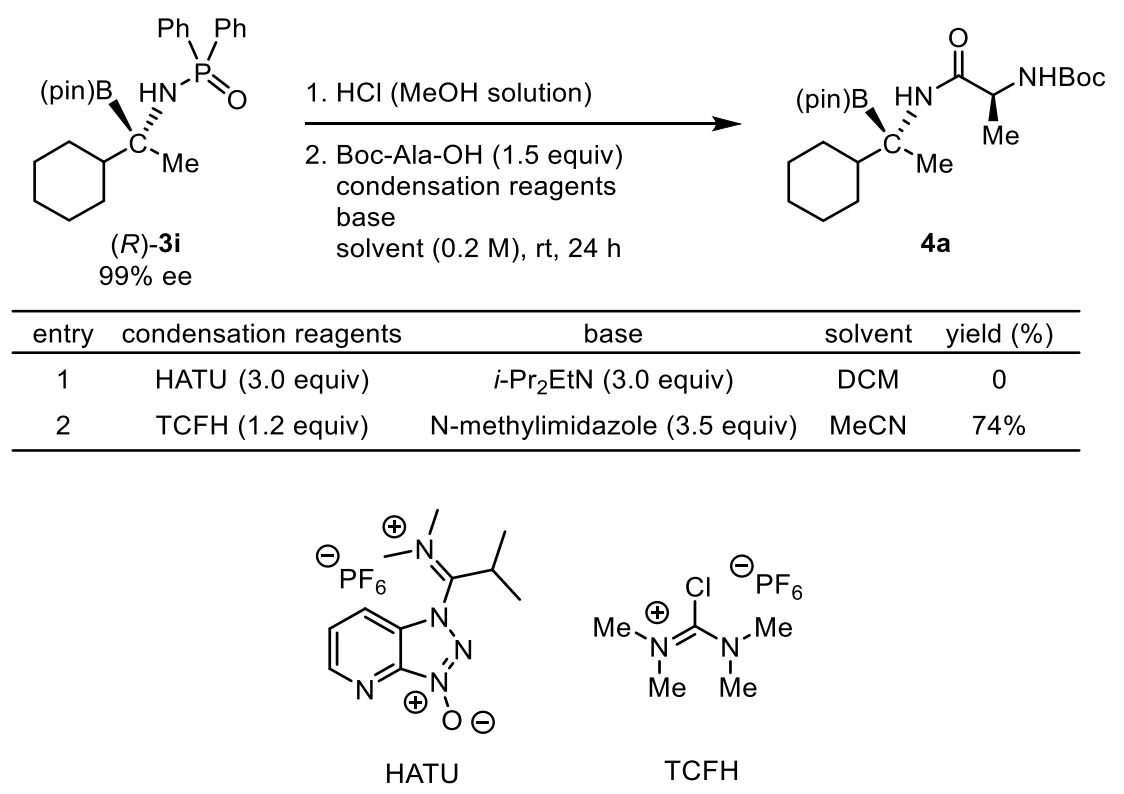

\section{Hydrolysis of 4 a with $\mathrm{NaIO}_{4}$}

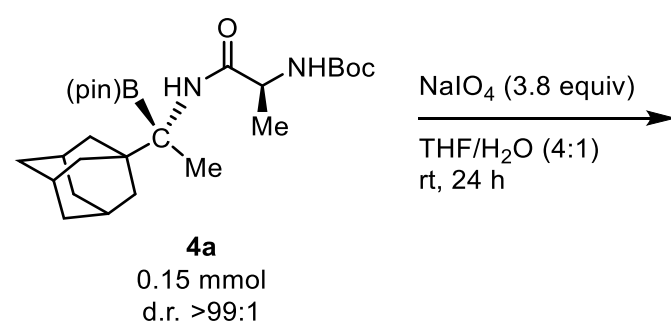<smiles>[R]B1OB([R])OB([R])O1</smiles>

5

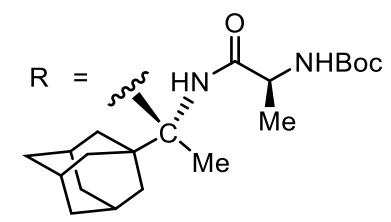

4a (71.6 mg, $0.15 \mathrm{mmol}, 1.0$ equiv) and $\mathrm{NaIO}_{4}(121.9 \mathrm{mg}, 0.57 \mathrm{mmol}, 3.8$ equiv) were placed in a vial with a screw cap containing a Teflon ${ }^{\circledR}$-coated rubber septum under air. THF $(1.0 \mathrm{~mL})$ and $\mathrm{H}_{2} \mathrm{O}$ 
$(250 \mu \mathrm{L})$ were added to the vial via a syringe. The resulting mixture was stirred for $24 \mathrm{~h}$ at room temperature. The reaction mixture was then diluted with brine and extracted with EtOAc three times, and dried over $\mathrm{MgSO}_{4}$, and concentrated under reduced pressure. The combined mixture was washed cold pentane to give the boroxine 5 in $70 \%$ yield $(41.3 \mathrm{mg}, 0.105 \mathrm{mmol})$ as white solid. The corresponding boronic acid was not detected. 


\section{Determination of Absolute Configuration of Borylation Product}

The absolute configuration of the product was determined based on X-ray crystallographic analysis of the compound $(R)$-3e. The absolute configurations of other borylation products were deduced by this product. The details were summarized in Figure S3 and Table S1.

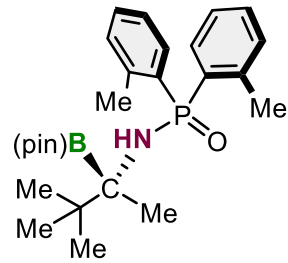

(R)-3e

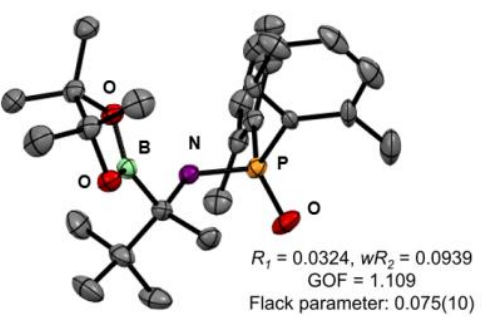

Figure S3. Molecular structure of $(R)$-3e. Thermal ellipsoids set at 50\% probability; hydrogen atoms omitted for clarity. 
Table S1. Summary of X-ray crystallographic data

\begin{tabular}{|c|c|}
\hline CCDC Name & 2052090 \\
\hline Empirical Formula & $\mathrm{C}_{52} \mathrm{H}_{78} \mathrm{~B}_{2} \mathrm{~N}_{2} \mathrm{O}_{6} \mathrm{P}_{2}$ \\
\hline Formula Weight & 910.72 \\
\hline Crystal System & monoclinic \\
\hline Crystal Size $/ \mathrm{mm}^{3}$ & $0.15 \times 0.15 \times 0.1$ \\
\hline$a / \AA$ & $7.83470(10)$ \\
\hline$b / \AA$ & $33.8529(3)$ \\
\hline$c / \AA$ & $10.56040(10)$ \\
\hline$\beta /{ }^{\circ}$ & $111.6360(10)$ \\
\hline$V / \AA 3$ & $2603.57(5)$ \\
\hline Space Group & $\mathrm{P} 2{ }_{1}$ \\
\hline$Z$ value & 2 \\
\hline$D$ calc $/ \mathrm{g} \mathrm{cm}^{-3}$ & 1.162 \\
\hline Temperature / K & 123 \\
\hline $2 \theta_{\max } /^{\circ}$ & 147.536 \\
\hline$\mu(\mathrm{MoK} \alpha) / \mathrm{cm}^{-1}$ & 11.32 \\
\hline Ne of Reflections & Total: 12604 \\
\hline Measured & $\begin{array}{l}\text { Unique: } 7917 \\
\left(R_{\text {int }}=0.0223\right)\end{array}$ \\
\hline $\begin{array}{l}\text { No. of Observations } \\
\text { (All reflections) }\end{array}$ & 7917 \\
\hline $\begin{array}{l}\text { Residuals: } R_{l} \\
(\mathrm{I}>2.00 \sigma(\mathrm{I}))\end{array}$ & 0.0324 \\
\hline $\begin{array}{l}\text { Residuals: } w R_{2} \\
\text { (All reflections) }\end{array}$ & 0.0939 \\
\hline $\begin{array}{l}\text { Goodness of Fit Indicator } \\
\qquad(\mathrm{GOF})\end{array}$ & 1.109 \\
\hline $\begin{array}{c}\text { Maximum Peak in } \\
\text { Final Diff. Map / } \AA^{3}\end{array}$ & $0.23 \mathrm{e}^{-}$ \\
\hline $\begin{array}{c}\text { Minimum Peak in } \\
\text { Final Diff. Map / } \AA^{3}\end{array}$ & $-0.24 \mathrm{e}^{-}$ \\
\hline Flack Parameter & $0.075(10)$ \\
\hline
\end{tabular}




\section{Plausible Catalytic Cycle}

Based on the theoretical and experimental results, we have proposed a plausible mechanism for the enantioselective borylation of aliphatic ketimines (Figure S4). First, $\mathrm{CuCl},(R, S)-\mathbf{L 1}$ and $\mathrm{Na}(\mathrm{O}-t-\mathrm{Bu})$ would react to form the copper(I)/NHC complex $\mathbf{A}$, which would undergo sigma-bond metathesis with $\mathbf{2}$ to give borylcopper(I) B. The subsequent insertion to $\mathbf{1}$ would lead to the formation of the $\operatorname{copper}(\mathrm{I})$ intermediate D, which would be protonated in the presence of an alcohol to afford the borylated product 3. This step would also result in the formation of copper alkoxide $\mathbf{A}$.

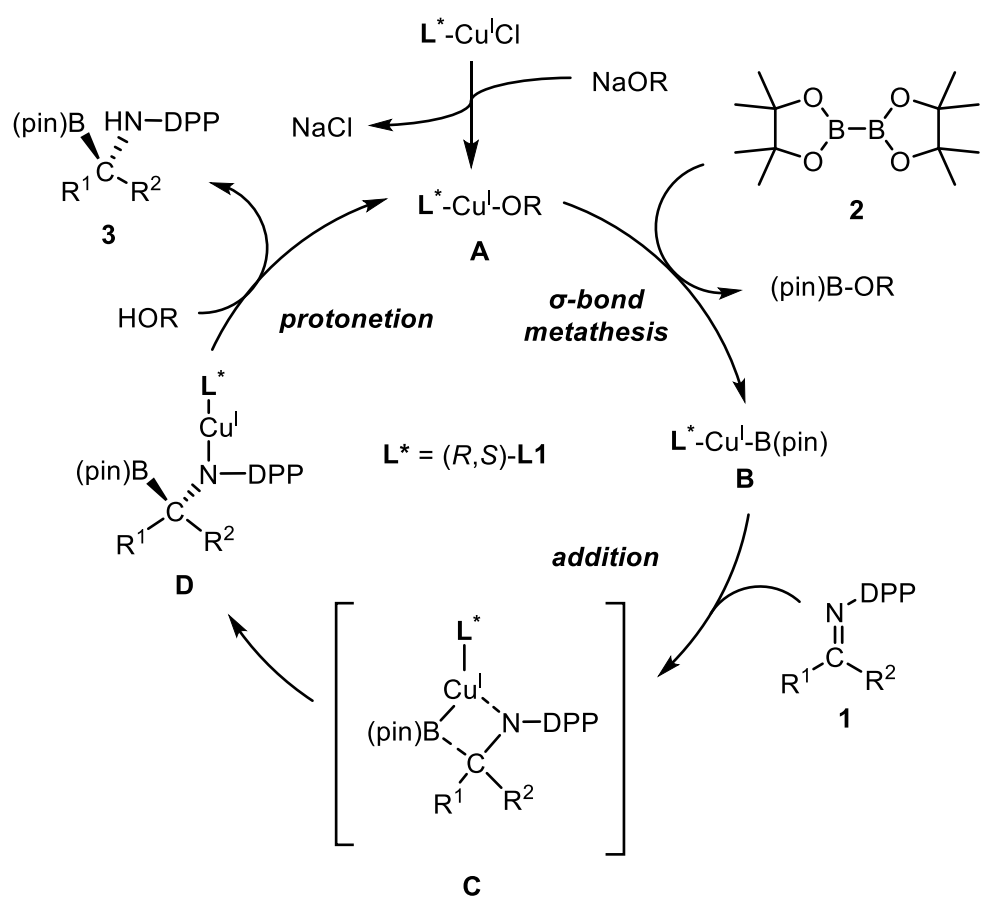

Figure S4. Proposed mechanism for the copper(I)-catalyzed enantioselective borylation of aliphatic ketimines. 


\section{DFT Calculations}

All calculations were performed using the Gaussian 09W (revision C.01) program package. ${ }^{8}$ Geometry optimizations and transition states (TS) calculations were performed with wB97XD/def2tzvp//wB97XD/Def2svp/THF(SMD). Molecular structures were drawn using the Mercury 3.5 program. ${ }^{9}$ Frequency calculations were conducted on gas-phase optimized geometries to check all the stationary points as either minima or transition states. Intrinsic reaction coordinate (IRC) calculations were carried out to confirm the transition state connecting the correct reactant and product on the potential energy surface.

We conducted the DFT calculations on the addition of the $(R, S)$-L1/borylcopper(I) complex (II) to ketimine 1p (I) to investigate the reaction mechanism and the origin of the enantioselectivity. The copper(I)-catalyzed borylation of dialkyl ketimine 1p proceeded to give $(R)-3 \mathbf{p}$ in $74 \%$ yield with $90 \%$ ee. As shown in Figure S3, The energy of the transition state (TS1) in the Si-face addition is lower than that of the transition state (TS3) in the Re-face addition by $1.93 \mathrm{kcal} / \mathrm{mol}$. This calculation result is in good agreement with the experimental result (calc. $92 \%$ ee versus exp. 90\% ee).<smiles>CC(C)C(=NP(=O)(c1ccccc1)c1ccccc1)c1ccccc1</smiles>

$1 \mathrm{p}$<smiles>CC1(C)OB(B2OC(C)(C)C(C)(C)O2)OC1(C)C</smiles>

2 (1.5 equiv)
$5 \mathrm{~mol} \% \mathrm{CuCl}$ $5 \mathrm{~mol} \%(R, S)-\mathrm{L} 1$

$\mathrm{Na}(\mathrm{O}-\mathrm{t}-\mathrm{Bu})$ (1.0 equiv.) $i$-PrOH (2.0 equiv.) $\operatorname{THF}(0.1 \mathrm{M}), \mathrm{rt}, 2 \mathrm{~h}$

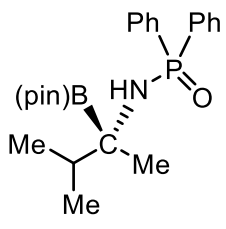

(R)-3p

$74 \%$ yield, $90 \%$ ee 


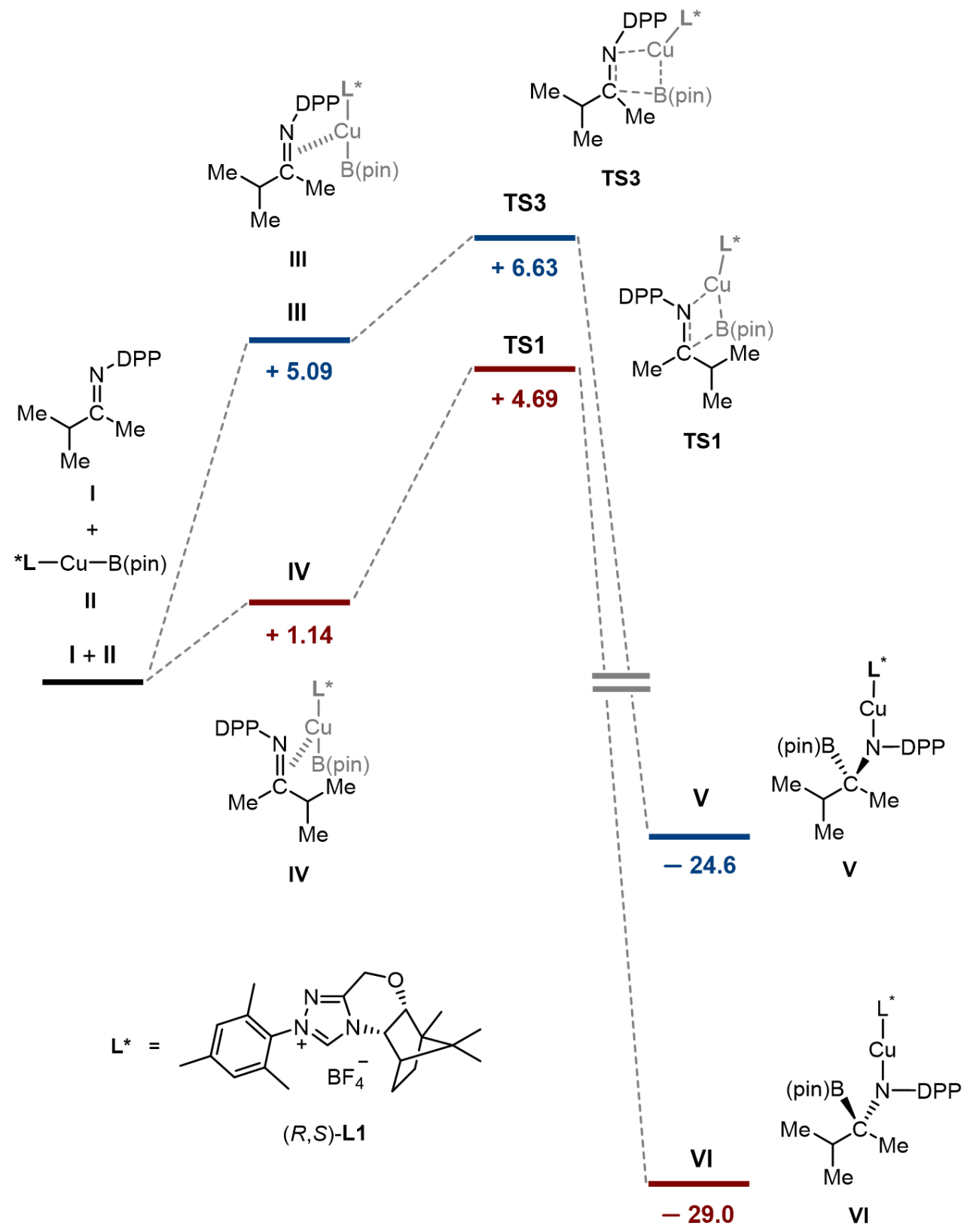

Figure S5. DFT calculations (wB97XD/def2tzvp//wB97XD/Def2svp THF(SMD)) of the addition of the $(R, S)-\mathbf{L} \mathbf{1} /$ borylcopper(I) complex to ketimine 1p. Gibbs free energy values relative to I and II are shown in $\mathrm{kcal} / \mathrm{mol}$ at $298 \mathrm{~K}$ and $1.0 \mathrm{~atm}$ in the gas phase. 


\section{Analysis for Non-Covalent Interactions in the Transition States}

Non-covalent interactions (NCIs) in the transition states were computed using the non-covalent interaction index from the optimized electron density at the same level of theory as the geometry optimizations. The wave function files (.wfn) were obtained from the corresponding formatted gaussian checkpoint files (.fchk) using the Multiwfn program. ${ }^{10}$ The following thresholds were applied to S33 to generate the NCI plot isosurface with NCIPLOT program, ${ }^{11,12} \operatorname{sign}(\lambda 2) \rho$ ranging from -0.2 to $0.2 \mathrm{au}$ and reduced density gradient $(\mathrm{RDG})=0.60 \mathrm{au}$. The surfaces were colored on a blue-greenred (BGR) scale using VMD program ${ }^{13}$ according to values of sign $(\lambda 2) \rho$ ranging from -0.01 to 0.01 au. The blue region indicates strong attractive interactions and the red region indicates strong repulsive interactions.

\section{Detailed Analysis of Transition State Structures}

The detailed analysis of both transition states TS1 and TS3 was carried out by the combination of structural analysis based on the transition state structures and the non-covalent interaction (NCI) plot. As shown in Figure $\mathrm{S} 6, \mathrm{C}\left(\mathrm{sp}_{3}\right)-\mathrm{H} / \pi$ interaction between the phenyl group of DPP and the methyl group of the mesityl group was found, and $\mathrm{C}\left(\mathrm{sp}_{3}\right)-\mathrm{H} / \mathrm{O}$ interaction between the camphor moiety and an oxygen atom of $\mathrm{P}=\mathrm{O}$ also existed. These interactions stabilize TS1 to afford the major enantiomer $(R)$ 3p. We also performed a non-covalent interaction (NCI) plot analysis of TS3. As shown Figure S7, an $\mathrm{C}\left(\mathrm{sp}_{3}\right)-\mathrm{H} / \pi$ interaction between the phenyl and the methyl group of the mesityl group was found. However, a steric hindrance between the isopropyl group of the substrate (1p) and camphor moiety might destabilize TS3 to produce minor enantiomer $(S)-\mathbf{3 p}$.
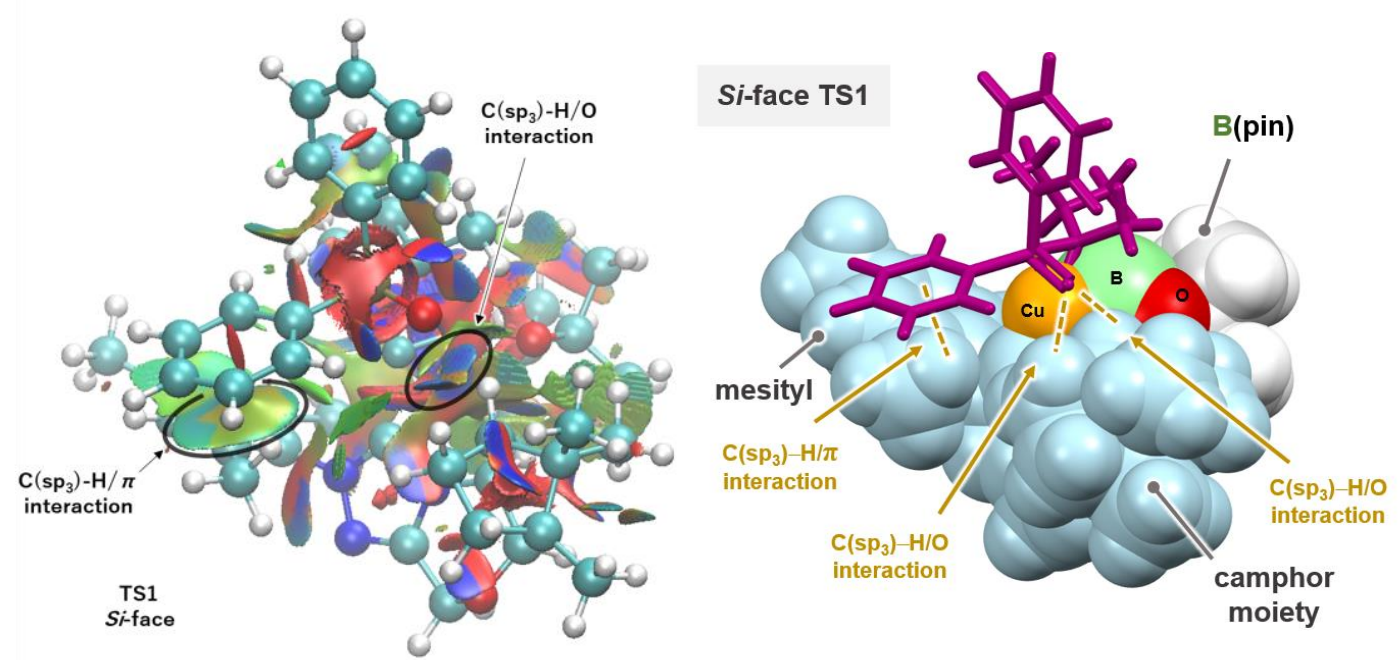

Figure S6. Detailed structural analysis of transition state structure for TS1. 

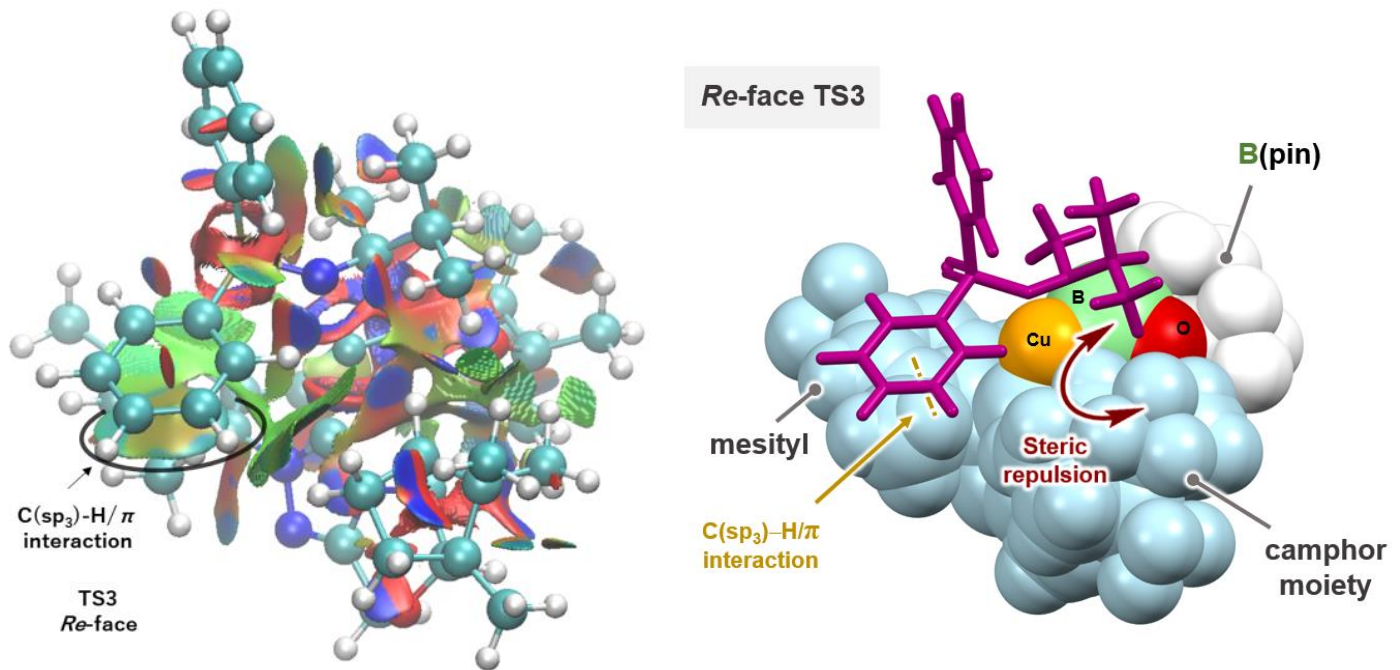

Figure S7. Detailed structural analysis of transition state structure for TS3. 


\section{Coordination profiles}

\section{Si-face TS1}

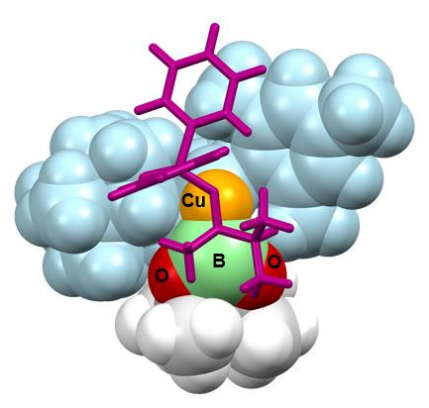

$\mathrm{Cu}$

$\begin{array}{lll}-0.06392 & 0.2486 & 0.59795\end{array}$

C

$\begin{array}{lll}2.87951 & -1.15702 & 3.23617\end{array}$

$\mathrm{O}$

$\begin{array}{lll}1.29464 & 0.5591 & 3.24174\end{array}$

$\mathrm{O}$

$\begin{array}{lll}2.01939 & -1.25353 & 2.08168\end{array}$

B

$\begin{array}{lll}1.04317 & -0.28889 & 2.18544\end{array}$

$\mathrm{C}$

$\begin{array}{lll}-1.0933 & -0.96821 & 1.84609\end{array}$

C

$\begin{array}{lll}-0.75084 & -2.44169 & 2.04232\end{array}$

C

$\begin{array}{lll}-1.68923 & -0.3093 & 3.11189\end{array}$

$\mathrm{H}$

$\begin{array}{lll}-0.9697 & -0.47892 & 3.92995\end{array}$

$\mathrm{H}$

$\begin{array}{lll}-0.14322 & -2.82482 & 1.21353\end{array}$

$\mathrm{N}$

$\begin{array}{lll}-1.83164 & -0.63708 & 0.67825\end{array}$

$\mathrm{P}$

$\begin{array}{lll}-2.18939 & -1.61919 & -0.58055\end{array}$

$\mathrm{O}$

$\begin{array}{lll}-1.14826 & -2.50003 & -1.23821\end{array}$

$\mathrm{H}$

$\begin{array}{lll}-1.68147 & -3.03196 & 2.07221\end{array}$

$\mathrm{H}$

$\begin{array}{lll}-0.21208 & -2.61301 & 2.98439\end{array}$

C

$\begin{array}{lll}-1.87627 & 1.19714 & 2.95777\end{array}$

$\mathrm{H}$

$\begin{array}{lll}-2.25155 & 1.64199 & 3.89294\end{array}$

$\mathrm{H}$

$\begin{array}{lll}-0.92457 & 1.69079 & 2.71302\end{array}$

$\mathrm{H}$

$\begin{array}{lll}-2.59698 & 1.42576 & 2.15807\end{array}$

C

$\begin{array}{lll}-3.01283 & -0.97346 & 3.5003\end{array}$

$\mathrm{H}$

$\begin{array}{lll}-3.75729 & -0.85034 & 2.69961\end{array}$

$\mathrm{H}$

$\begin{array}{lll}-2.90109 & -2.05063 & 3.69406\end{array}$

$\mathrm{H}$

$\begin{array}{lll}-3.42005 & -0.51345 & 4.41461\end{array}$

C

$\begin{array}{lll}2.64568 & 0.3261 & 3.68469\end{array}$ 


\begin{tabular}{|c|c|c|c|}
\hline $\mathrm{C}$ & 2.72898 & 0.56528 & 5.18235 \\
\hline $\mathrm{H}$ & 3.73022 & 0.30918 & 5.56097 \\
\hline $\mathrm{H}$ & 2.54517 & 1.6281 & 5.39851 \\
\hline $\mathrm{H}$ & 1.98424 & -0.02586 & 5.73095 \\
\hline $\mathrm{C}$ & 3.54084 & 1.31665 & 2.9395 \\
\hline $\mathrm{H}$ & 3.48757 & 1.16075 & 1.85083 \\
\hline $\mathrm{H}$ & 3.20309 & 2.34105 & 3.15271 \\
\hline $\mathrm{H}$ & 4.59057 & 1.23045 & 3.25488 \\
\hline $\mathrm{C}$ & 2.38469 & -2.17532 & 4.26024 \\
\hline $\mathrm{H}$ & 2.366 & -3.17123 & 3.79408 \\
\hline $\mathrm{H}$ & 3.04698 & -2.21731 & 5.13727 \\
\hline $\mathrm{H}$ & 1.36656 & -1.94044 & 4.60419 \\
\hline $\mathrm{C}$ & 4.30811 & -1.47579 & 2.82866 \\
\hline $\mathrm{H}$ & 4.39611 & -2.53753 & 2.55622 \\
\hline $\mathrm{H}$ & 4.62922 & -0.87542 & 1.96714 \\
\hline $\mathrm{H}$ & 4.99762 & -1.28291 & 3.66464 \\
\hline $\mathrm{C}$ & -2.92196 & -0.458 & -1.78315 \\
\hline $\mathrm{C}$ & -2.7738 & -0.74164 & -3.14484 \\
\hline $\mathrm{C}$ & -3.64742 & 0.67577 & -1.39414 \\
\hline $\mathrm{C}$ & -3.35071 & 0.08938 & -4.10613 \\
\hline $\mathrm{H}$ & -2.19729 & -1.62052 & -3.44393 \\
\hline $\mathrm{C}$ & -4.23789 & 1.49549 & -2.35389 \\
\hline $\mathrm{H}$ & -3.73851 & 0.91763 & -0.33312 \\
\hline $\mathrm{C}$ & -4.09152 & 1.20311 & -3.71116 \\
\hline $\mathrm{H}$ & -3.22276 & -0.13496 & -5.16791 \\
\hline $\mathrm{H}$ & -4.8079 & 2.37357 & -2.04091 \\
\hline $\mathrm{H}$ & -4.54893 & 1.85123 & -4.4629 \\
\hline $\mathrm{C}$ & -3.58862 & -2.6952 & -0.06787 \\
\hline $\mathrm{C}$ & -4.76665 & -2.18143 & 0.48862 \\
\hline $\mathrm{C}$ & -3.45406 & -4.07902 & -0.21977 \\
\hline $\mathrm{C}$ & -5.78903 & -3.03837 & 0.89285 \\
\hline $\mathrm{H}$ & -4.8908 & -1.10191 & 0.60691 \\
\hline $\mathrm{C}$ & -4.47413 & -4.9389 & 0.18943 \\
\hline
\end{tabular}




\begin{tabular}{|c|c|c|c|}
\hline $\mathrm{H}$ & -2.53434 & -4.46926 & -0.66186 \\
\hline $\mathrm{C}$ & -5.64227 & -4.41959 & 0.74712 \\
\hline $\mathrm{H}$ & -6.70419 & -2.62731 & 1.32606 \\
\hline $\mathrm{H}$ & -4.35672 & -6.01876 & 0.06896 \\
\hline $\mathrm{H}$ & -6.44235 & -5.09178 & 1.06711 \\
\hline $\mathrm{C}$ & 1.05626 & 1.3705 & -0.47651 \\
\hline $\mathrm{N}$ & 2.25734 & 1.06015 & -1.01757 \\
\hline $\mathrm{N}$ & 0.95613 & 2.68375 & -0.72206 \\
\hline $\mathrm{C}$ & 2.90352 & -0.23962 & -1.01321 \\
\hline $\mathrm{C}$ & 2.81041 & 2.18623 & -1.55525 \\
\hline $\mathrm{N}$ & 2.02569 & 3.20851 & -1.39189 \\
\hline $\mathrm{C}$ & -0.18498 & 3.47823 & -0.39031 \\
\hline $\mathrm{C}$ & 2.00813 & -1.41187 & -1.42676 \\
\hline $\mathrm{C}$ & 4.07547 & -0.29638 & -2.05083 \\
\hline $\mathrm{H}$ & 3.2779 & -0.40376 & 0.00536 \\
\hline $\mathrm{C}$ & 4.12657 & 2.05888 & -2.25142 \\
\hline $\mathrm{C}$ & -1.19505 & 3.61619 & -1.34804 \\
\hline $\mathrm{C}$ & -0.2726 & 4.03412 & 0.89315 \\
\hline $\mathrm{C}$ & 1.3816 & -1.10722 & -2.79548 \\
\hline $\mathrm{H}$ & 1.27373 & -1.69966 & -0.66295 \\
\hline $\mathrm{C}$ & 3.08429 & -2.47912 & -1.76319 \\
\hline $\mathrm{C}$ & 3.76583 & -1.58502 & -2.85557 \\
\hline $\mathrm{H}$ & 5.03832 & -0.38654 & -1.51727 \\
\hline $\mathrm{O}$ & 4.15116 & 0.82125 & -2.90888 \\
\hline $\mathrm{H}$ & 4.95292 & 2.14859 & -1.51998 \\
\hline $\mathrm{H}$ & 4.24131 & 2.85378 & -2.99879 \\
\hline $\mathrm{C}$ & -2.333 & 4.34318 & -0.98605 \\
\hline $\mathrm{C}$ & -1.06632 & 2.9688 & -2.69813 \\
\hline $\mathrm{C}$ & -1.43266 & 4.7408 & 1.21109 \\
\hline $\mathrm{C}$ & 0.84101 & 3.84775 & 1.88586 \\
\hline $\mathrm{H}$ & 0.56848 & -1.8184 & -2.9837 \\
\hline $\mathrm{H}$ & 0.9258 & -0.10712 & -2.82631 \\
\hline $\mathrm{C}$ & 2.571 & -1.25371 & -3.77617 \\
\hline
\end{tabular}




\begin{tabular}{|c|c|c|c|}
\hline $\mathrm{C}$ & 3.99312 & -2.86037 & -0.58601 \\
\hline $\mathrm{C}$ & 2.48647 & -3.78478 & -2.29473 \\
\hline $\mathrm{C}$ & 4.97948 & -2.14996 & -3.56714 \\
\hline $\mathrm{C}$ & -2.47207 & 4.90357 & 0.28619 \\
\hline $\mathrm{H}$ & -3.13443 & 4.46573 & -1.71957 \\
\hline $\mathrm{H}$ & -0.08247 & 3.16706 & -3.14862 \\
\hline $\mathrm{H}$ & -1.17723 & 1.8749 & -2.61247 \\
\hline $\mathrm{H}$ & -1.8416 & 3.33167 & -3.38515 \\
\hline $\mathrm{H}$ & -1.5301 & 5.177 & 2.20948 \\
\hline $\mathrm{H}$ & 0.96767 & 2.78568 & 2.14883 \\
\hline $\mathrm{H}$ & 1.79981 & 4.19825 & 1.47251 \\
\hline $\mathrm{H}$ & 0.63921 & 4.40246 & 2.81204 \\
\hline $\mathrm{H}$ & 2.41367 & -2.07237 & -4.49362 \\
\hline $\mathrm{H}$ & 2.76183 & -0.34367 & -4.3578 \\
\hline $\mathrm{H}$ & 3.38 & -3.19474 & 0.26458 \\
\hline $\mathrm{H}$ & 4.65749 & -3.69095 & -0.87102 \\
\hline $\mathrm{H}$ & 4.63819 & -2.04944 & -0.22287 \\
\hline $\mathrm{H}$ & 3.2815 & -4.45514 & -2.65777 \\
\hline $\mathrm{H}$ & 1.96073 & -4.30631 & -1.4795 \\
\hline $\mathrm{H}$ & 1.76411 & -3.65019 & -3.10928 \\
\hline $\mathrm{H}$ & 4.72393 & -3.06684 & -4.12059 \\
\hline $\mathrm{H}$ & 5.37879 & -1.42285 & -4.29172 \\
\hline $\mathrm{H}$ & 5.78539 & -2.3934 & -2.85637 \\
\hline $\mathrm{C}$ & -3.70785 & 5.6745 & 0.66571 \\
\hline $\mathrm{H}$ & -3.46886 & 6.73253 & 0.8584 \\
\hline $\mathrm{H}$ & -4.46665 & 5.63813 & -0.12834 \\
\hline $\mathrm{H}$ & -4.15599 & 5.27062 & 1.58668 \\
\hline
\end{tabular}




\section{Si-face TS2}

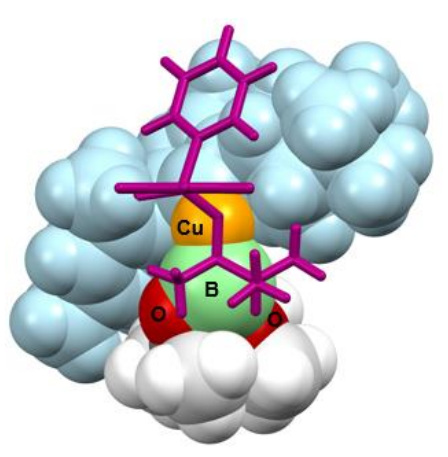

\begin{tabular}{|c|c|c|c|}
\hline $\mathrm{Cu}$ & -0.32131 & 0.33266 & 0.12578 \\
\hline $\mathrm{C}$ & -3.96906 & 1.77265 & 1.73222 \\
\hline $\mathrm{O}$ & -2.17371 & 0.44923 & 2.44747 \\
\hline $\mathrm{O}$ & -2.91943 & 1.75152 & 0.74534 \\
\hline B & -1.8472 & 1.06861 & 1.26116 \\
\hline $\mathrm{C}$ & 0.00828 & 2.12728 & 1.09581 \\
\hline $\mathrm{C}$ & -0.58113 & 3.40828 & 0.5008 \\
\hline $\mathrm{C}$ & 0.22592 & 2.24726 & 2.62941 \\
\hline $\mathrm{H}$ & -0.76223 & 2.4074 & 3.08942 \\
\hline $\mathrm{H}$ & -0.9553 & 3.24566 & -0.51597 \\
\hline $\mathrm{N}$ & 1.17075 & 1.62204 & 0.43376 \\
\hline $\mathrm{P}$ & 1.81529 & 2.15062 & -0.97157 \\
\hline $\mathrm{O}$ & 0.93987 & 2.56239 & -2.13733 \\
\hline $\mathrm{H}$ & 0.21027 & 4.17287 & 0.44004 \\
\hline $\mathrm{H}$ & -1.39661 & 3.80546 & 1.11877 \\
\hline $\mathrm{C}$ & 0.82759 & 0.98938 & 3.24379 \\
\hline $\mathrm{H}$ & 0.92095 & 1.09965 & 4.3356 \\
\hline $\mathrm{H}$ & 0.19547 & 0.11143 & 3.05054 \\
\hline $\mathrm{H}$ & 1.82951 & 0.79856 & 2.83034 \\
\hline $\mathrm{C}$ & 1.08432 & 3.46835 & 2.97296 \\
\hline $\mathrm{H}$ & 2.064 & 3.41597 & 2.47756 \\
\hline $\mathrm{H}$ & 0.60774 & 4.41482 & 2.6795 \\
\hline $\mathrm{H}$ & 1.25841 & 3.51316 & 4.05962 \\
\hline $\mathrm{C}$ & -3.60029 & 0.53551 & 2.62565 \\
\hline
\end{tabular}




\begin{tabular}{|c|c|c|c|}
\hline $\mathrm{C}$ & -3.9048 & 0.7004 & 4.10512 \\
\hline $\mathrm{H}$ & -4.9815 & 0.86444 & 4.26371 \\
\hline $\mathrm{H}$ & -3.61701 & -0.21259 & 4.64743 \\
\hline $\mathrm{H}$ & -3.35358 & 1.543 & 4.5427 \\
\hline $\mathrm{C}$ & -4.19401 & -0.7681 & 2.09964 \\
\hline $\mathrm{H}$ & -4.00431 & -0.87203 & 1.02353 \\
\hline $\mathrm{H}$ & -3.71757 & -1.61545 & 2.61447 \\
\hline $\mathrm{H}$ & -5.27869 & -0.82288 & 2.27202 \\
\hline $\mathrm{C}$ & -3.86647 & 3.1022 & 2.47768 \\
\hline $\mathrm{H}$ & -3.93986 & 3.92588 & 1.75254 \\
\hline $\mathrm{H}$ & -4.67833 & 3.21609 & 3.21063 \\
\hline $\mathrm{H}$ & -2.90646 & 3.19888 & 3.00589 \\
\hline $\mathrm{C}$ & -5.31289 & 1.67373 & 1.0286 \\
\hline $\mathrm{H}$ & -5.48675 & 2.58276 & 0.43393 \\
\hline $\mathrm{H}$ & -5.34785 & 0.81372 & 0.34846 \\
\hline $\mathrm{H}$ & -6.12854 & 1.58446 & 1.7621 \\
\hline $\mathrm{C}$ & 2.8889 & 0.75213 & -1.47507 \\
\hline $\mathrm{C}$ & 3.98509 & 0.31246 & -0.72354 \\
\hline $\mathrm{C}$ & 2.57985 & 0.09628 & -2.67174 \\
\hline $\mathrm{C}$ & 4.76109 & -0.75828 & -1.16452 \\
\hline $\mathrm{H}$ & 4.24506 & 0.80789 & 0.21377 \\
\hline $\mathrm{C}$ & 3.33966 & -0.993 & -3.1011 \\
\hline $\mathrm{H}$ & 1.73442 & 0.45313 & -3.26437 \\
\hline $\mathrm{C}$ & 4.43533 & -1.41957 & -2.34997 \\
\hline $\mathrm{H}$ & 5.62176 & -1.08282 & -0.57523 \\
\hline $\mathrm{H}$ & 3.08268 & -1.50164 & -4.03372 \\
\hline $\mathrm{H}$ & 5.04073 & -2.26307 & -2.69108 \\
\hline $\mathrm{C}$ & 2.97009 & 3.53167 & -0.61436 \\
\hline $\mathrm{C}$ & 3.01574 & 4.60534 & -1.51083 \\
\hline $\mathrm{C}$ & 3.8029 & 3.55108 & 0.51212 \\
\hline $\mathrm{C}$ & 3.88277 & 5.67611 & -1.29046 \\
\hline $\mathrm{H}$ & 2.3547 & 4.58753 & -2.38049 \\
\hline $\mathrm{C}$ & 4.67406 & 4.61709 & 0.73092 \\
\hline
\end{tabular}




\begin{tabular}{|c|c|c|c|}
\hline $\mathrm{H}$ & 3.76759 & 2.72963 & 1.23128 \\
\hline $\mathrm{C}$ & 4.71531 & 5.68167 & -0.17122 \\
\hline $\mathrm{H}$ & 3.9084 & 6.50955 & -1.99692 \\
\hline $\mathrm{H}$ & 5.32032 & 4.62005 & 1.6122 \\
\hline $\mathrm{H}$ & 5.39754 & 6.5179 & 0.00092 \\
\hline $\mathrm{C}$ & -0.48573 & -1.261 & -0.957 \\
\hline $\mathrm{N}$ & 0.37088 & -2.31302 & -0.95943 \\
\hline $\mathrm{N}$ & -1.34851 & -1.59058 & -1.93319 \\
\hline $\mathrm{C}$ & 1.51098 & -2.53597 & -0.08492 \\
\hline $\mathrm{C}$ & -0.01044 & -3.20012 & -1.92444 \\
\hline $\mathrm{N}$ & -1.07413 & -2.78294 & -2.53857 \\
\hline $\mathrm{C}$ & -2.57064 & -0.90495 & -2.22373 \\
\hline $\mathrm{C}$ & 1.26752 & -2.20355 & 1.39305 \\
\hline $\mathrm{C}$ & 1.91451 & -4.05329 & -0.0484 \\
\hline $\mathrm{H}$ & 2.33978 & -1.93429 & -0.47718 \\
\hline $\mathrm{C}$ & 0.74492 & -4.4817 & -2.04738 \\
\hline $\mathrm{C}$ & -3.75288 & -1.42717 & -1.67415 \\
\hline $\mathrm{C}$ & -2.54881 & 0.25243 & -3.0067 \\
\hline $\mathrm{C}$ & -0.0052 & -2.92562 & 1.86445 \\
\hline $\mathrm{H}$ & 1.28865 & -1.12419 & 1.58661 \\
\hline $\mathrm{C}$ & 2.39702 & -3.02299 & 2.07551 \\
\hline $\mathrm{C}$ & 1.91038 & -4.38001 & 1.46441 \\
\hline $\mathrm{H}$ & 2.92652 & -4.16895 & -0.47449 \\
\hline $\mathrm{O}$ & 1.04607 & -4.91458 & -0.74727 \\
\hline $\mathrm{H}$ & 1.66192 & -4.33382 & -2.64913 \\
\hline $\mathrm{H}$ & 0.12628 & -5.24313 & -2.53777 \\
\hline $\mathrm{C}$ & -4.93449 & -0.72108 & -1.89026 \\
\hline $\mathrm{C}$ & -3.73731 & -2.70792 & -0.88522 \\
\hline $\mathrm{C}$ & -3.7607 & 0.93234 & -3.18253 \\
\hline $\mathrm{C}$ & -1.27921 & 0.75168 & -3.63347 \\
\hline $\mathrm{H}$ & -0.31261 & -2.55428 & 2.85147 \\
\hline $\mathrm{H}$ & -0.85731 & -2.74941 & 1.19425 \\
\hline $\mathrm{C}$ & 0.42979 & -4.41194 & 1.90615 \\
\hline
\end{tabular}




\begin{tabular}{|c|c|c|c|}
\hline $\mathrm{C}$ & 3.81816 & -2.61484 & 1.67103 \\
\hline $\mathrm{C}$ & 2.34097 & -2.96062 & 3.60503 \\
\hline $\mathrm{C}$ & 2.6989 & -5.62801 & 1.80939 \\
\hline $\mathrm{C}$ & -4.9544 & 0.47021 & -2.62766 \\
\hline $\mathrm{H}$ & -5.86513 & -1.10475 & -1.46184 \\
\hline $\mathrm{H}$ & -3.52896 & -3.56771 & -1.54103 \\
\hline $\mathrm{H}$ & -2.95361 & -2.69523 & -0.11189 \\
\hline $\mathrm{H}$ & -4.70396 & -2.87847 & -0.39295 \\
\hline $\mathrm{H}$ & -3.76499 & 1.85007 & -3.7774 \\
\hline $\mathrm{H}$ & -0.65848 & 1.33408 & -2.92962 \\
\hline $\mathrm{H}$ & -0.66468 & -0.08477 & -3.99953 \\
\hline $\mathrm{H}$ & -1.50563 & 1.408 & -4.48557 \\
\hline $\mathrm{H}$ & 0.34718 & -4.836 & 2.91716 \\
\hline $\mathrm{H}$ & -0.16507 & -5.0469 & 1.23801 \\
\hline $\mathrm{H}$ & 3.99595 & -1.55902 & 1.92871 \\
\hline $\mathrm{H}$ & 4.55688 & -3.21939 & 2.21976 \\
\hline $\mathrm{H}$ & 4.03626 & -2.72899 & 0.60227 \\
\hline $\mathrm{H}$ & 3.09901 & -3.62818 & 4.04357 \\
\hline $\mathrm{H}$ & 2.56852 & -1.93684 & 3.94242 \\
\hline $\mathrm{H}$ & 1.36975 & -3.23789 & 4.03384 \\
\hline $\mathrm{H}$ & 2.69163 & -5.81394 & 2.89455 \\
\hline $\mathrm{H}$ & 2.2636 & -6.51089 & 1.31589 \\
\hline $\mathrm{H}$ & 3.74774 & -5.544 & 1.48329 \\
\hline $\mathrm{C}$ & -6.24768 & 1.21448 & -2.8221 \\
\hline $\mathrm{H}$ & -6.74846 & 1.39169 & -1.85774 \\
\hline $\mathrm{H}$ & -6.08591 & 2.18654 & -3.30836 \\
\hline $\mathrm{H}$ & -6.94402 & 0.63316 & -3.44752 \\
\hline
\end{tabular}




\section{$R e$-face TS3}

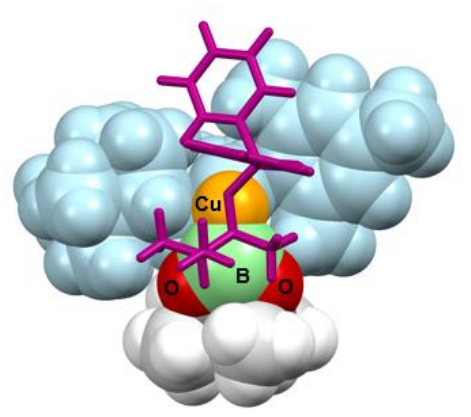

\begin{tabular}{|c|c|c|c|}
\hline $\mathrm{Cu}$ & 0.00263 & -0.39312 & 0.29451 \\
\hline $\mathrm{C}$ & 2.9024 & -2.96928 & 1.70614 \\
\hline $\mathrm{C}$ & 1.83232 & -3.22448 & 2.82873 \\
\hline $\mathrm{O}$ & 0.79172 & -2.29673 & 2.46943 \\
\hline $\mathrm{O}$ & 2.09154 & -2.47938 & 0.61919 \\
\hline B & 0.91833 & -1.97684 & 1.14087 \\
\hline $\mathrm{C}$ & -1.01164 & -2.13846 & 0.0175 \\
\hline $\mathrm{C}$ & 2.29988 & -2.91306 & 4.24125 \\
\hline $\mathrm{H}$ & 2.58272 & -1.85827 & 4.35433 \\
\hline $\mathrm{H}$ & 1.48909 & -3.12307 & 4.95437 \\
\hline $\mathrm{H}$ & 3.16223 & -3.54065 & 4.51321 \\
\hline $\mathrm{C}$ & 1.22586 & -4.62505 & 2.77261 \\
\hline $\mathrm{H}$ & 1.94758 & -5.39054 & 3.09213 \\
\hline $\mathrm{H}$ & 0.35951 & -4.66695 & 3.44892 \\
\hline $\mathrm{H}$ & 0.8783 & -4.87405 & 1.7592 \\
\hline $\mathrm{C}$ & 3.89462 & -1.86518 & 2.06939 \\
\hline $\mathrm{H}$ & 4.56937 & -2.17905 & 2.87863 \\
\hline $\mathrm{H}$ & 4.51277 & -1.63201 & 1.18954 \\
\hline $\mathrm{H}$ & 3.37694 & -0.94591 & 2.38249 \\
\hline $\mathrm{C}$ & 3.65056 & -4.20885 & 1.24363 \\
\hline $\mathrm{H}$ & 4.37571 & -3.93535 & 0.46292 \\
\hline $\mathrm{H}$ & 4.20433 & -4.66233 & 2.0796 \\
\hline $\mathrm{H}$ & 2.96876 & -4.96058 & 0.82517 \\
\hline $\mathrm{C}$ & -0.54247 & -3.30203 & -0.89109 \\
\hline $\mathrm{C}$ & -1.83718 & -2.63445 & 1.20427 \\
\hline
\end{tabular}




\begin{tabular}{|c|c|c|c|}
\hline $\mathrm{H}$ & -2.84384 & -2.91815 & 0.85765 \\
\hline $\mathrm{H}$ & -1.37263 & -3.51133 & 1.67492 \\
\hline $\mathrm{H}$ & -1.96698 & -1.85338 & 1.96213 \\
\hline $\mathrm{C}$ & 0.35999 & -2.85155 & -2.03194 \\
\hline $\mathrm{H}$ & -0.15099 & -2.10497 & -2.65868 \\
\hline $\mathrm{H}$ & 1.28852 & -2.41391 & -1.64311 \\
\hline $\mathrm{H}$ & 0.63549 & -3.70564 & -2.67039 \\
\hline $\mathrm{H}$ & 0.03207 & -3.99981 & -0.25816 \\
\hline $\mathrm{C}$ & -1.75154 & -4.06485 & -1.44414 \\
\hline $\mathrm{H}$ & -1.4167 & -4.88559 & -2.09788 \\
\hline $\mathrm{H}$ & -2.37064 & -4.50763 & -0.6507 \\
\hline $\mathrm{H}$ & -2.39284 & -3.39876 & -2.04108 \\
\hline $\mathrm{N}$ & -1.55126 & -1.04831 & -0.7182 \\
\hline $\mathrm{P}$ & -2.97752 & -0.28642 & -0.47111 \\
\hline $\mathrm{O}$ & -3.51718 & -0.01347 & 0.91276 \\
\hline $\mathrm{C}$ & -4.2424 & -1.22675 & -1.41343 \\
\hline $\mathrm{C}$ & -5.34927 & -1.74439 & -0.73501 \\
\hline $\mathrm{C}$ & -4.10422 & -1.47259 & -2.78554 \\
\hline $\mathrm{C}$ & -6.30107 & -2.50751 & -1.41434 \\
\hline $\mathrm{H}$ & -5.45319 & -1.53997 & 0.33347 \\
\hline $\mathrm{C}$ & -5.05785 & -2.22558 & -3.46708 \\
\hline $\mathrm{H}$ & -3.24074 & -1.073 & -3.32493 \\
\hline $\mathrm{C}$ & -6.15649 & -2.74833 & -2.78018 \\
\hline $\mathrm{H}$ & -7.16134 & -2.91284 & -0.87571 \\
\hline $\mathrm{H}$ & -4.94299 & -2.41017 & -4.53802 \\
\hline $\mathrm{H}$ & -6.90234 & -3.3426 & -3.31399 \\
\hline $\mathrm{C}$ & -2.79406 & 1.26576 & -1.41588 \\
\hline $\mathrm{C}$ & -1.79963 & 1.46869 & -2.37896 \\
\hline $\mathrm{C}$ & -3.70916 & 2.29103 & -1.14979 \\
\hline $\mathrm{C}$ & -1.72686 & 2.67785 & -3.07092 \\
\hline $\mathrm{H}$ & -1.0732 & 0.67599 & -2.56904 \\
\hline $\mathrm{C}$ & -3.64279 & 3.49592 & -1.84802 \\
\hline $\mathrm{H}$ & -4.46997 & 2.13974 & -0.37981 \\
\hline
\end{tabular}




\begin{tabular}{|c|c|c|c|}
\hline $\mathrm{C}$ & -2.65164 & 3.69045 & -2.81104 \\
\hline $\mathrm{H}$ & -0.94366 & 2.83071 & -3.81775 \\
\hline $\mathrm{H}$ & -4.36179 & 4.29024 & -1.63288 \\
\hline $\mathrm{H}$ & -2.59445 & 4.6368 & -3.35449 \\
\hline $\mathrm{C}$ & 0.98903 & 1.18742 & 0.7657 \\
\hline $\mathrm{N}$ & 2.25213 & 1.45414 & 0.35305 \\
\hline $\mathrm{N}$ & 0.76137 & 2.16988 & 1.64932 \\
\hline $\mathrm{C}$ & 3.04305 & 0.66262 & -0.56743 \\
\hline $\mathrm{C}$ & 2.71797 & 2.55731 & 1.00574 \\
\hline $\mathrm{N}$ & 1.81463 & 3.02795 & 1.81045 \\
\hline $\mathrm{C}$ & -0.46767 & 2.39549 & 2.34493 \\
\hline $\mathrm{C}$ & 2.36558 & 0.31876 & -1.89422 \\
\hline $\mathrm{C}$ & 4.34014 & 1.41423 & -1.00873 \\
\hline $\mathrm{H}$ & 3.2942 & -0.26572 & -0.04376 \\
\hline $\mathrm{C}$ & 4.10077 & 3.0336 & 0.69625 \\
\hline $\mathrm{C}$ & -1.36785 & 3.32526 & 1.80829 \\
\hline $\mathrm{C}$ & -0.72595 & 1.68269 & 3.52143 \\
\hline $\mathrm{C}$ & 1.92485 & 1.61492 & -2.59206 \\
\hline $\mathrm{H}$ & 1.57083 & -0.42798 & -1.77539 \\
\hline $\mathrm{C}$ & 3.60299 & -0.13141 & -2.71811 \\
\hline $\mathrm{C}$ & 4.32701 & 1.24942 & -2.552 \\
\hline $\mathrm{H}$ & 5.22499 & 0.92565 & -0.56323 \\
\hline $\mathrm{O}$ & 4.36283 & 2.77997 & -0.65683 \\
\hline $\mathrm{H}$ & 4.82995 & 2.52333 & 1.35496 \\
\hline $\mathrm{H}$ & 4.18125 & 4.1138 & 0.87062 \\
\hline $\mathrm{C}$ & -2.57405 & 3.52281 & 2.48162 \\
\hline $\mathrm{C}$ & -1.03868 & 4.06435 & 0.54208 \\
\hline $\mathrm{C}$ & -1.94846 & 1.91324 & 4.15751 \\
\hline $\mathrm{C}$ & 0.27044 & 0.69857 & 4.06955 \\
\hline $\mathrm{H}$ & 1.24092 & 1.39215 & -3.42255 \\
\hline $\mathrm{H}$ & 1.37656 & 2.27923 & -1.90876 \\
\hline $\mathrm{C}$ & 3.25912 & 2.22757 & -3.08475 \\
\hline $\mathrm{C}$ & 4.36556 & -1.3275 & -2.12795 \\
\hline
\end{tabular}




\begin{tabular}{|c|c|c|c|}
\hline $\mathrm{C}$ & 3.25388 & -0.50077 & -4.16218 \\
\hline $\mathrm{C}$ & 5.6968 & 1.40416 & -3.18277 \\
\hline $\mathrm{C}$ & -2.88446 & 2.82007 & 3.6505 \\
\hline $\mathrm{H}$ & -3.2945 & 4.23871 & 2.076 \\
\hline $\mathrm{H}$ & -0.94531 & 3.36387 & -0.30324 \\
\hline $\mathrm{H}$ & -1.82231 & 4.79187 & 0.29499 \\
\hline $\mathrm{H}$ & -0.0818 & 4.60109 & 0.6314 \\
\hline $\mathrm{H}$ & -2.17534 & 1.36606 & 5.07697 \\
\hline $\mathrm{H}$ & 0.27255 & -0.23696 & 3.48743 \\
\hline $\mathrm{H}$ & 1.29275 & 1.10579 & 4.03986 \\
\hline $\mathrm{H}$ & 0.03333 & 0.44348 & 5.11156 \\
\hline $\mathrm{H}$ & 3.30901 & 2.27281 & -4.18208 \\
\hline $\mathrm{H}$ & 3.42698 & 3.24252 & -2.70624 \\
\hline $\mathrm{H}$ & 3.67901 & -2.17447 & -1.97568 \\
\hline $\mathrm{H}$ & 5.15144 & -1.6556 & -2.82537 \\
\hline $\mathrm{H}$ & 4.85726 & -1.1296 & -1.16563 \\
\hline $\mathrm{H}$ & 4.1697 & -0.65209 & -4.75468 \\
\hline $\mathrm{H}$ & 2.69447 & -1.44994 & -4.17336 \\
\hline $\mathrm{H}$ & 2.64032 & 0.2463 & -4.6817 \\
\hline $\mathrm{H}$ & 5.64651 & 1.27764 & -4.2753 \\
\hline $\mathrm{H}$ & 6.10625 & 2.40638 & -2.98131 \\
\hline $\mathrm{H}$ & 6.40985 & 0.66415 & -2.78555 \\
\hline $\mathrm{C}$ & -4.21641 & 3.00305 & 4.32548 \\
\hline $\mathrm{H}$ & -4.62556 & 4.00855 & 4.15077 \\
\hline $\mathrm{H}$ & -4.94444 & 2.276 & 3.92918 \\
\hline $\mathrm{H}$ & -4.14477 & 2.83898 & 5.41047 \\
\hline
\end{tabular}




\section{$R e$-face TS4}

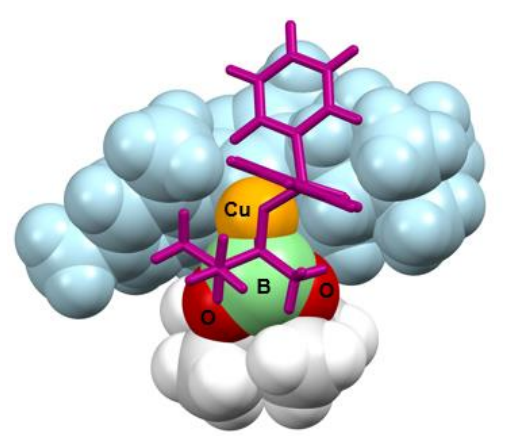

$\mathrm{Cu}$

$\begin{array}{llll}0.38065 & -0.55231 & -0.04231\end{array}$

C

C

$\mathrm{O}$

$\begin{array}{lll}3.52854 & -1.39344 & 2.52424\end{array}$

$\mathrm{O}$

$\begin{array}{lll}2.52477 & -0.7389 & 3.5422\end{array}$

B

$\begin{array}{lll}1.41266 & -0.39829 & 2.69086\end{array}$

$\mathrm{C}$

$\begin{array}{lll}2.64439 & -1.88684 & 1.499\end{array}$

$\begin{array}{lll}1.4829 & -1.15425 & 1.54584\end{array}$

C

$\begin{array}{lll}-0.3318 & -2.22476 & 0.88826\end{array}$

C

$\begin{array}{lll}3.03356 & 0.52759 & 4.21313\end{array}$

$\mathrm{H}$

$3.24747 \quad 1.31777 \quad 3.48203$

$\mathrm{H}$

$2.27479 \quad 0.90776 \quad 4.91342$

$\mathrm{H}$

$\begin{array}{lll}3.95074 & 0.32158 \quad 4.78573\end{array}$

$\mathrm{C}$

$\begin{array}{lll}2.0172 & -1.71817 \quad 4.59772\end{array}$

$\mathrm{H}$

$2.79153 \quad-1.9335 \quad 5.34845$

$\mathrm{H}$

$\begin{array}{lll}1.15147 & -1.27447 \quad 5.11087\end{array}$

$\mathrm{H}$

$\begin{array}{lll}1.69688 & -2.66963 & 4.15061\end{array}$

C

$\begin{array}{lll}4.45092 & -0.37328 & 1.8624\end{array}$

$\mathrm{H}$

$5.20822 \quad 0.00641 \quad 2.56354$

$\mathrm{H}$

$\begin{array}{lll}4.96605 & -0.85137 & 1.01643\end{array}$

$\mathrm{H}$

$\begin{array}{lll}3.87661 & 0.47696 \quad 1.4702\end{array}$

C

$\begin{array}{lll}4.34001 & -2.55185 & 3.08058\end{array}$

$\mathrm{H}$

$\begin{array}{lll}5.01272 & -2.94134 & 2.30207\end{array}$

$\mathrm{H}$

$\begin{array}{lll}4.95832 & -2.21948 \quad 3.9283\end{array}$

$\mathrm{H}$

$\begin{array}{lll}3.69771 & -3.37606 & 3.41711\end{array}$

C

$\begin{array}{lll}0.33576 & -3.55452 & 0.45823\end{array}$ 


$$
\begin{aligned}
& \begin{array}{lll}
-0.87487 & -2.29046 \quad 2.31458
\end{array} \\
& \begin{array}{lll}
-1.79029 & -2.90455 & 2.32512
\end{array} \\
& \begin{array}{lll}
-0.15084 & -2.75663 & 2.99673
\end{array} \\
& \begin{array}{lll}
-1.14269 & -1.30013 \quad 2.69659
\end{array} \\
& \begin{array}{lll}
1.00493 & -3.46617 & -0.9086
\end{array} \\
& \begin{array}{lll}
0.25454 & -3.31206 & -1.69884
\end{array} \\
& \begin{array}{lll}
1.72737 & -2.63581 & -0.9477
\end{array} \\
& \begin{array}{lll}
1.55305 & -4.39395 & -1.13601
\end{array} \\
& \begin{array}{lll}
1.11664 & -3.77454 & 1.20228
\end{array} \\
& \begin{array}{lll}
-0.67676 & -4.70327 & 0.47867
\end{array} \\
& \begin{array}{lll}
-0.19685 & -5.64039 & 0.15476
\end{array} \\
& \begin{array}{lll}
-1.09507 & -4.87782 & 1.48093
\end{array} \\
& -1.51498 \quad-4.49886 \quad-0.20508 \\
& \begin{array}{lll}
-1.22323 & -1.73135 & -0.1068
\end{array} \\
& \begin{array}{lll}
-2.72202 & -1.12849 & 0.13375
\end{array} \\
& \begin{array}{lll}
-3.07552 & -0.33534 & 1.37463
\end{array} \\
& \begin{array}{lll}
-3.89392 & -2.53087 & -0.0084
\end{array} \\
& \begin{array}{lll}
-4.83775 & -2.73368 & 1.00229
\end{array} \\
& \begin{array}{lll}
-3.85159 & -3.41265 & -1.09609
\end{array} \\
& \begin{array}{lll}
-5.7269 & -3.8077 & 0.93175
\end{array} \\
& \begin{array}{lll}
-4.86262 & -2.03704 & 1.84371
\end{array} \\
& -4.74252-4.48129-1.17065 \\
& \begin{array}{lll}
-3.11311 & -3.26263 & -1.8888
\end{array} \\
& \begin{array}{lll}
-5.68059 & -4.68105 & -0.15441
\end{array} \\
& \begin{array}{lll}
-6.46126 & -3.96102 & 1.72659
\end{array} \\
& \begin{array}{lll}
-4.70538 & -5.1653 & -2.02211
\end{array} \\
& -6.37866-5.51995-0.21188 \\
& \begin{array}{lll}
-3.04404 & -0.10929 & -1.3512
\end{array} \\
& \begin{array}{lll}
-2.2237 & -0.11824 & -2.48302
\end{array} \\
& \begin{array}{lll}
-4.11795 & 0.78797 & -1.29572
\end{array} \\
& \begin{array}{lll}
-2.44662 & 0.77938 & -3.52698
\end{array} \\
& \begin{array}{lll}
-1.3914 & -0.8196 & -2.52731
\end{array} \\
& \begin{array}{lll}
-4.34631 & 1.68305 & -2.33979
\end{array}
\end{aligned}
$$




\begin{tabular}{|c|c|c|c|}
\hline $\mathrm{H}$ & -4.75979 & 0.80399 & -0.41173 \\
\hline $\mathrm{C}$ & -3.50303 & 1.68825 & -3.45269 \\
\hline $\mathrm{H}$ & -1.78961 & 0.77173 & -4.40039 \\
\hline $\mathrm{H}$ & -5.17879 & 2.38841 & -2.28051 \\
\hline $\mathrm{H}$ & -3.67381 & 2.39855 & -4.26546 \\
\hline $\mathrm{C}$ & 0.87977 & 1.01722 & -1.04683 \\
\hline $\mathrm{N}$ & 0.23587 & 2.20905 & -1.05832 \\
\hline $\mathrm{N}$ & 1.83065 & 1.19317 & -1.98048 \\
\hline $\mathrm{C}$ & -0.89716 & 2.60929 & -0.23959 \\
\hline $\mathrm{C}$ & 0.81372 & 3.02274 & -1.99026 \\
\hline $\mathrm{N}$ & 1.80767 & 2.42424 & -2.57138 \\
\hline $\mathrm{C}$ & 2.8774 & 0.26574 & -2.28094 \\
\hline $\mathrm{C}$ & -0.78703 & 2.25676 & 1.25013 \\
\hline $\mathrm{C}$ & -1.0698 & 4.16889 & -0.22924 \\
\hline $\mathrm{H}$ & -1.78652 & 2.13669 & -0.67375 \\
\hline $\mathrm{C}$ & 0.29486 & 4.41521 & -2.13459 \\
\hline $\mathrm{C}$ & 4.15208 & 0.51048 & -1.75293 \\
\hline $\mathrm{C}$ & 2.59234 & -0.85804 & -3.06601 \\
\hline $\mathrm{C}$ & 0.53806 & 2.81199 & 1.79568 \\
\hline $\mathrm{H}$ & -0.97286 & 1.19285 & 1.45173 \\
\hline $\mathrm{C}$ & -1.84494 & 3.22756 & 1.84651 \\
\hline $\mathrm{C}$ & -1.13715 & 4.50335 & 1.28028 \\
\hline $\mathrm{H}$ & -2.01523 & 4.43187 & -0.73487 \\
\hline $\mathrm{O}$ & -0.0259 & 4.88258 & -0.85285 \\
\hline $\mathrm{H}$ & -0.58553 & 4.42684 & -2.80597 \\
\hline $\mathrm{H}$ & 1.06555 & 5.06701 & -2.56403 \\
\hline $\mathrm{C}$ & 5.14904 & -0.43618 & -1.99962 \\
\hline $\mathrm{C}$ & 4.44155 & 1.76158 & -0.97127 \\
\hline $\mathrm{C}$ & 3.61973 & -1.7794 & -3.27888 \\
\hline $\mathrm{C}$ & 1.22092 & -1.07249 & -3.63847 \\
\hline $\mathrm{H}$ & 0.74399 & 2.38998 & 2.78737 \\
\hline $\mathrm{H}$ & 1.39374 & 2.53255 & 1.16504 \\
\hline $\mathrm{C}$ & 0.29533 & 4.34178 & 1.83435 \\
\hline
\end{tabular}




$\begin{array}{lccc}\mathrm{C} & -3.2737 & 2.99837 & 1.33498 \\ \mathrm{C} & -1.91244 & 3.16569 & 3.37519 \\ \mathrm{C} & -1.78017 & 5.84657 & 1.56416 \\ \mathrm{C} & 4.89876 & -1.59228 & -2.74547 \\ \mathrm{H} & 6.14839 & -0.26768 & -1.58835 \\ \mathrm{H} & 4.49822 & 2.6326 & -1.64314 \\ \mathrm{H} & 3.65005 & 1.97483 & -0.23728 \\ \mathrm{H} & 5.39711 & 1.67887 & -0.43672 \\ \mathrm{H} & 3.41177 & -2.6704 & -3.87803 \\ \mathrm{H} & 0.51605 & -1.35296 & -2.84254 \\ \mathrm{H} & 0.83534 & -0.15707 & -4.11174 \\ \mathrm{H} & 1.22588 & -1.8784 & -4.384 \\ \mathrm{H} & 0.34706 & 4.73895 & 2.85853 \\ \mathrm{H} & 1.01683 & 4.90527 & 1.22983 \\ \mathrm{H} & -3.54221 & 1.94251 & 1.49226 \\ \mathrm{H} & -3.98214 & 3.63401 & 1.88894 \\ \mathrm{H} & -3.41533 & 3.21826 & 0.26797 \\ \mathrm{H} & -2.57041 & 3.95914 & 3.76339 \\ \mathrm{H} & -2.34387 & 2.20086 & 3.68425 \\ \mathrm{H} & -0.94052 & 3.26641 & 3.8751 \\ \mathrm{H} & -1.82961 & 6.04245 & 2.6465 \\ \mathrm{H} & -1.20116 & 6.66168 & 1.10225 \\ \mathrm{H} & -2.80463 & 5.89402 & 1.16156 \\ \mathrm{H} & 5.9722 & -2.62754 & -2.94714 \\ \mathrm{H} & 5.87728 & -3.12013 & -3.9257 \\ \mathrm{H} & 6.97664 & -2.18667 & -2.87411 \\ \mathrm{H} & 5.89813 & -3.41213 & -2.17609\end{array}$




\section{Reference}

(1) Shintani, R.; Takatsu, K.; Takeda, M.; Hayashi, T. Copper-Catalyzed Asymmetric Allylic Substitution of Allyl Phosphates with Aryl- and Alkenylboronates. Angew. Chem., Int. Ed. 2011, $50,8656-8659$.

(2) Winn, C. L.; Guillen, F.; Pytkowicz, J.; Roland, S.; Mangeney, P.; Alexakis, A. Enantioselective copper catalysed 1,4-conjugate addition reactions using chiral N-heterocyclic carbenes. J. Org. Met. Chem. 2005, 690, 5672-5695.

(3) Harada, A.; Makida, Y.; Sato, T.; Ohmiya, H.; Sawamura, M. Copper-Catalyzed Enantioselective Allylic Alkylation of Terminal Alkyne Pronucleophiles. J. Am. Chem. Soc. 2014, 136, 1393213939.

(4) Núñez, M. G.; Farley, A. J. M.; Dixon, D. J. Bifunctional Iminophosphorane Organocatalysts for Enantioselective Synthesis: Application to the Ketimine Nitro-Mannich Reaction. J. Am. Chem. Soc. 2013, 135, 16348-16351.

(5) Abell, J. P.; Yamamoto, H. Dual-Activation Asymmetric Strecker Reaction of Aldimines and Ketimines Catalyzed by a Tethered Bis(8-quinolinolato) Aluminum Complex. J. Am. Chem. Soc. 2009, 131, 15118-15119.

(6) Masumoto, S., Usuda, H., Suzuki, M., Kanai, M., \& Shibasaki, M. Catalytic Enantioselective Strecker Reaction of Ketoimines. J. Am. Chem. Soc. 2003, 125, 5634-5635.

(7) Beutner, G. L.; Young, I. S.; Davies, M. L.; Hickey, M. R.; Park, H.; Stevens, J. M.; Ye, Q. TCFHNMI: Direct Access to N-Acyl Imidazoliums for Challenging Amide Bond Formations. Org. Lett. 2018, 20, 4218-4222.

(8) Gaussian 09, Revision C.01, Frisch, M. J.; Trucks, G. W.; Schlegel, H. B.; Scuseria, G. E.; Robb, M. A.; Cheeseman, J. R.; Scalmani, G.; Barone, V.; Mennucci, B.; Petersson, G. A.; Nakatsuji, H.; Caricato, M.; Li, X.; Hratchian, H. P.; Izmaylov, A. F.; Bloino, J.; Zheng, G.; Sonnenberg, J. L.; Hada, M.; Ehara, M.; Toyota, K.; Fukuda, R.; Hasegawa, J.; Ishida, M.; Nakajima, T.; Honda, Y.; Kitao, O.; Nakai, H.; Vreven, T.; Montgomery, J., J. A.; Peralta, J. E.; Ogliaro, F.; Bearpark, M.; Heyd, J. J.; Brothers, E.; Kudin, K. N.; Staroverov, V. N.; Kobayashi, R.; Normand, J.; Raghavachari, K.; Rendell, A.; Burant, J. C.; Iyengar, S. S.; Tomasi, J.; Cossi, M.; Rega, N.; Millam, N. J.; Klene, M.; Knox, J. E.; Cross, J. B.; Bakken, V.; Adamo, C.; Jaramillo, J.; Gomperts, R.; Stratmann, R. E.; Yazyev, O.; Austin, A. J.; Cammi, R.; Pomelli, C.; Ochterski, J. W.; Martin, R. L.; Morokuma, K.; Zakrzewski, V. G.; Voth, G. A.; Salvador, P.; Dannenberg, J. J.; Dapprich, S.; Daniels, A. D.; Farkas, Ö.; Foresman, J. B.; Ortiz, J. V.; Cioslowski, J.; Fox, D. J.; Gaussian, Inc., Wallingford CT, 2009. 
(9) Mercury: http://www.ccdc.cam.ac.uk/Solutions/CSDSystem/Pages/Mercury.aspx

(10)Lu, T.; Chen, F. Multiwfn: A multifunctional wavefunction analyzer. J. Comput. Chem. 2012, $33,580-592$.

(11)Johnson, E. R.; Keinan, S.; Mori-Sánchez, P.; Contreras-García, J.; Cohen, A. J.; Yang, W. Revealing Noncovalent Interactions. J. Am. Chem. Soc. 2010, 132, 6498-6506.

(12)Contreras-García, J.; Johnson, E. R.; Keinan, S.; Chaudret, R.; Piquemal, J. P.; Beratan, D. N.; Yang, W. NCIPLOT: A Program for Plotting Noncovalent Interaction Regions. J. Chem. Theory Comput. 2011, 7, 625-632.

(13)Humphrey, W.; Dalke, A.; Schulten, K. VMD: Visual molecular dynamics. J. Mol. Graph. 1996, $14,33-38$. 


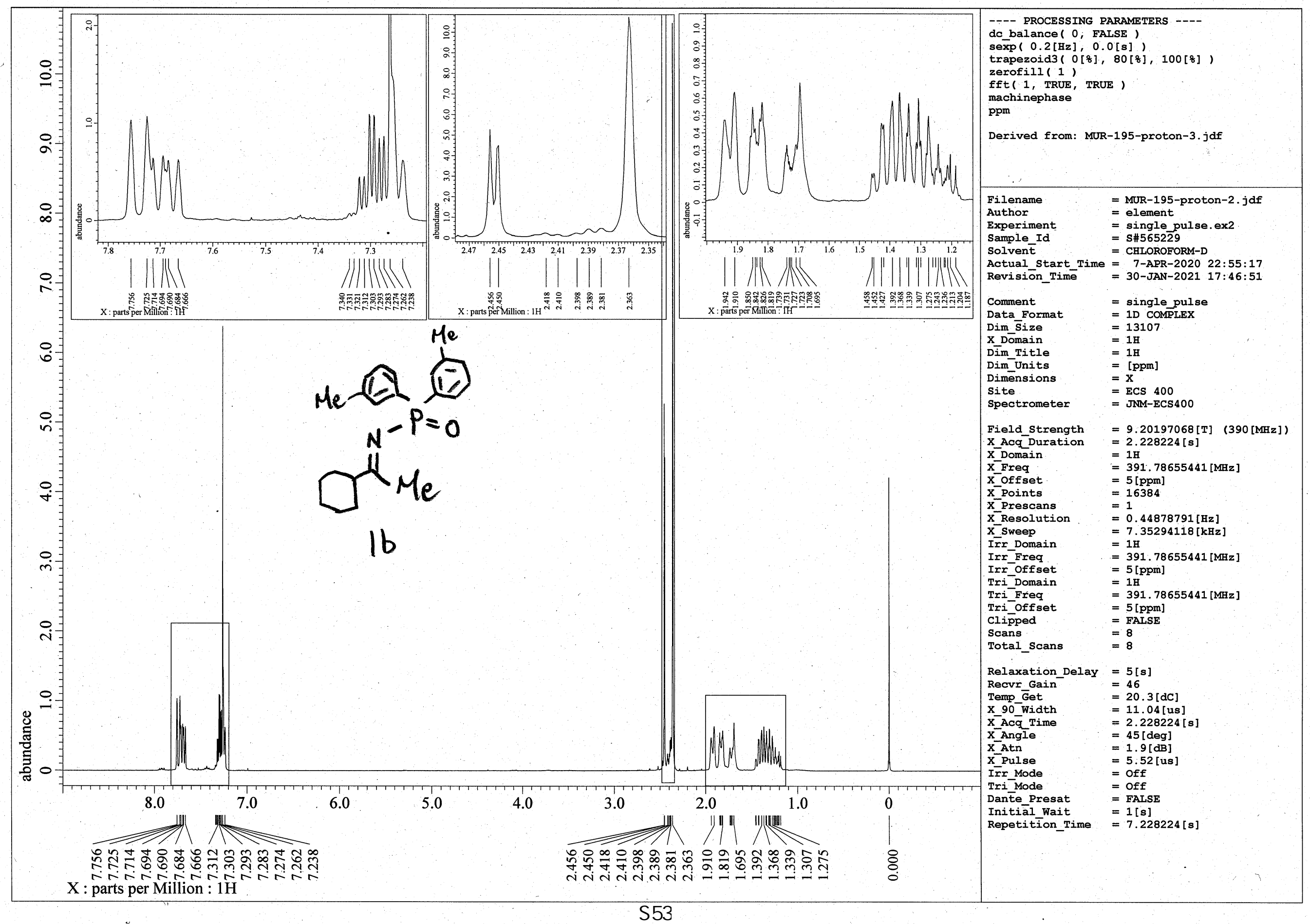




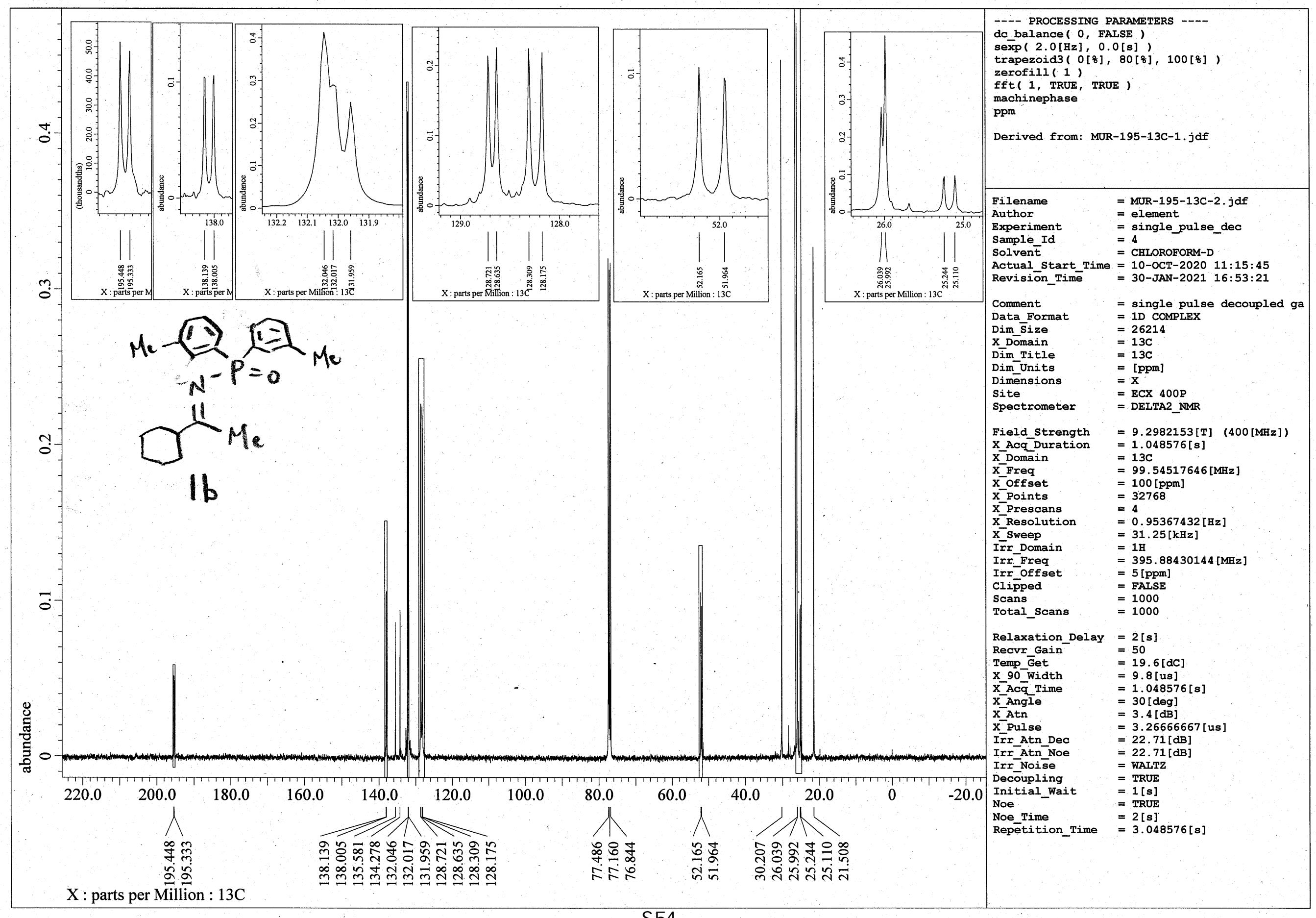




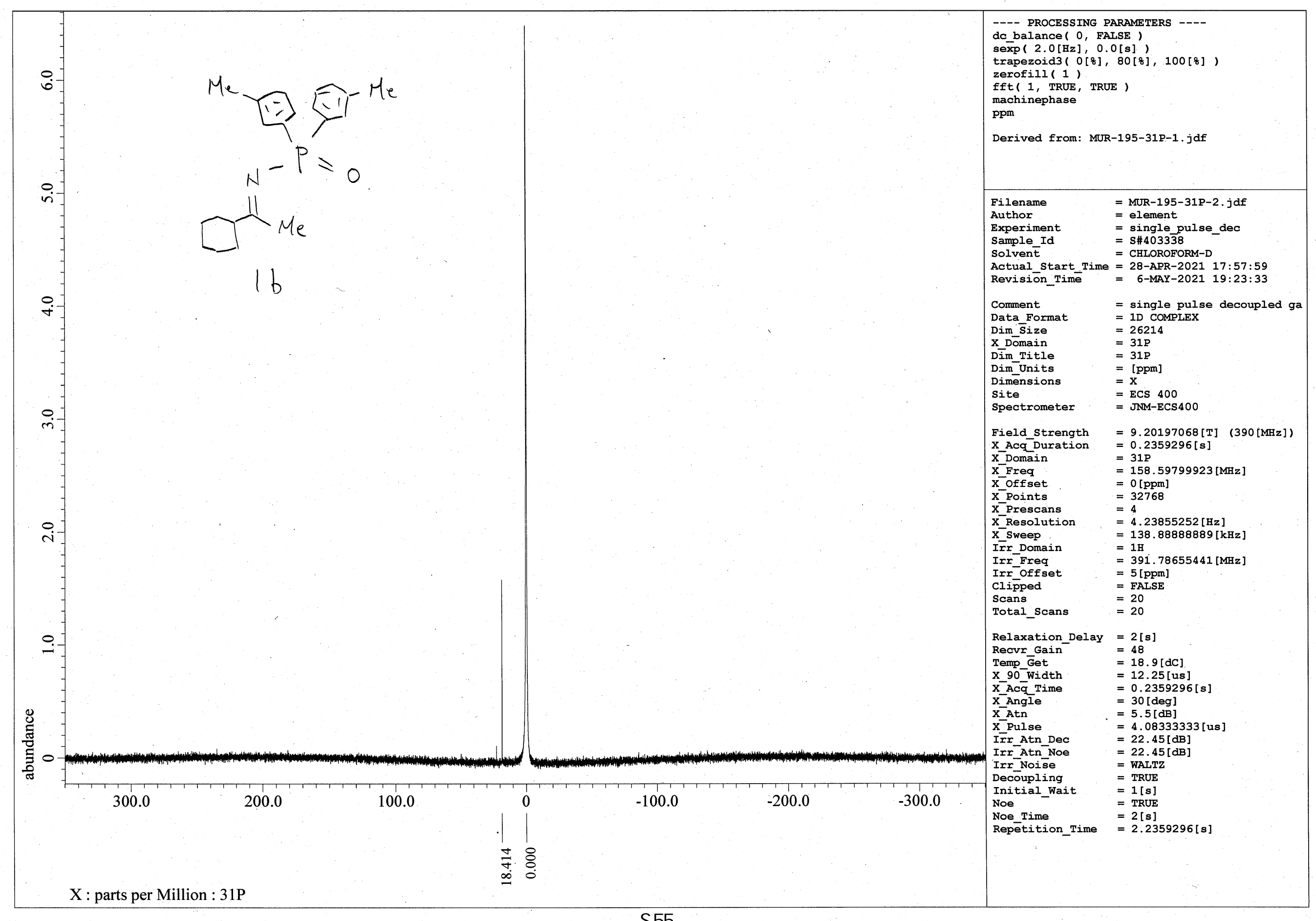




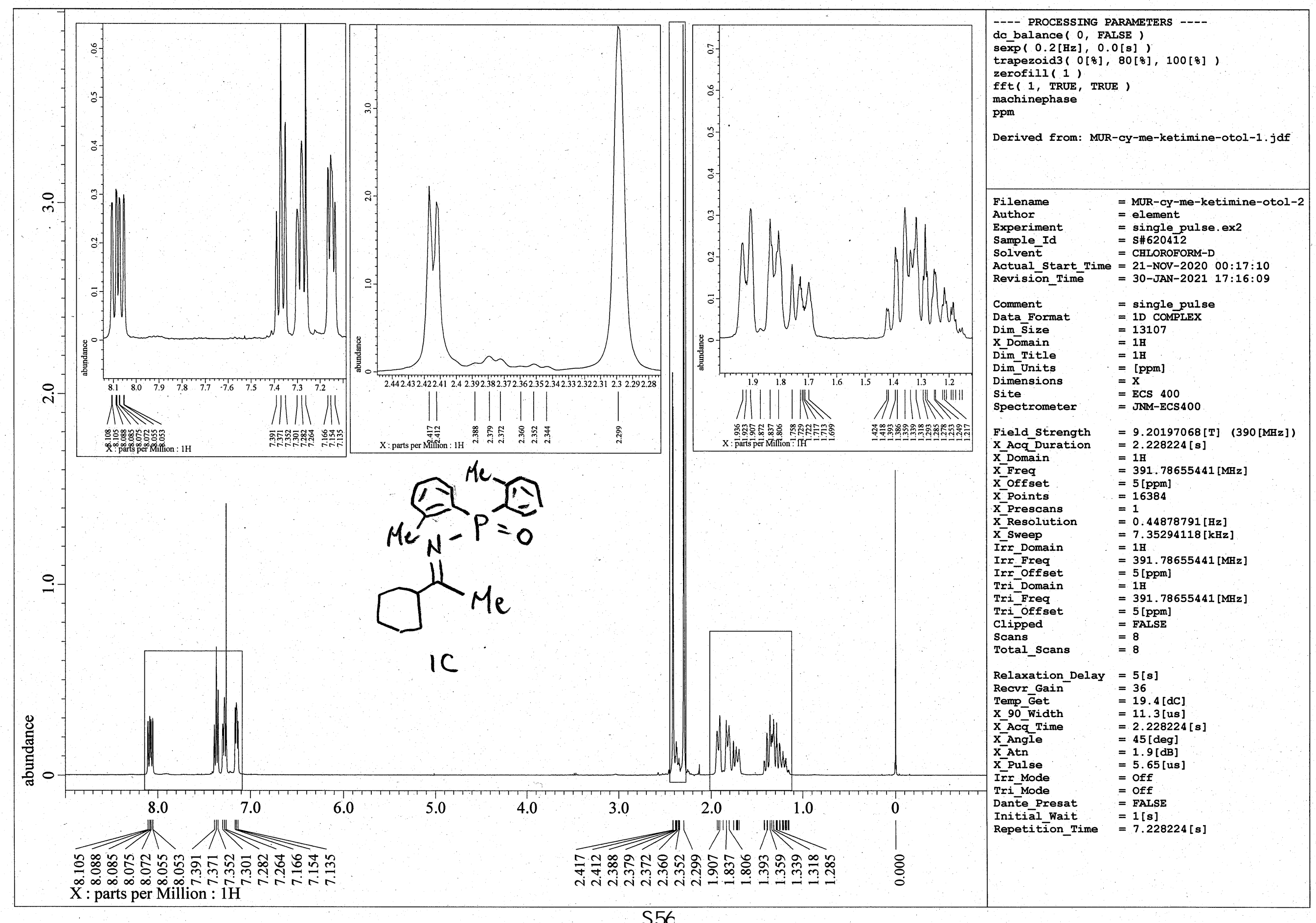




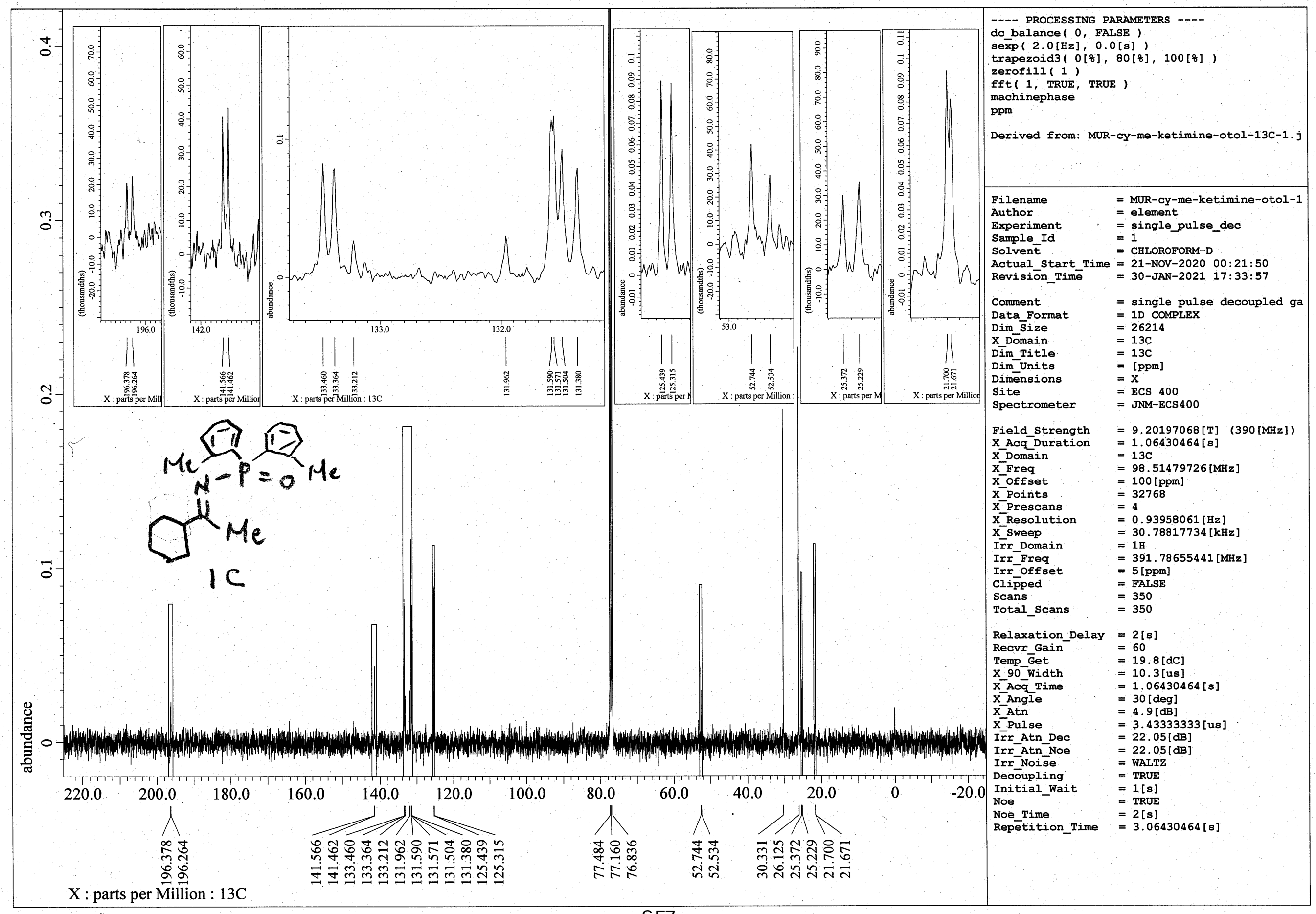




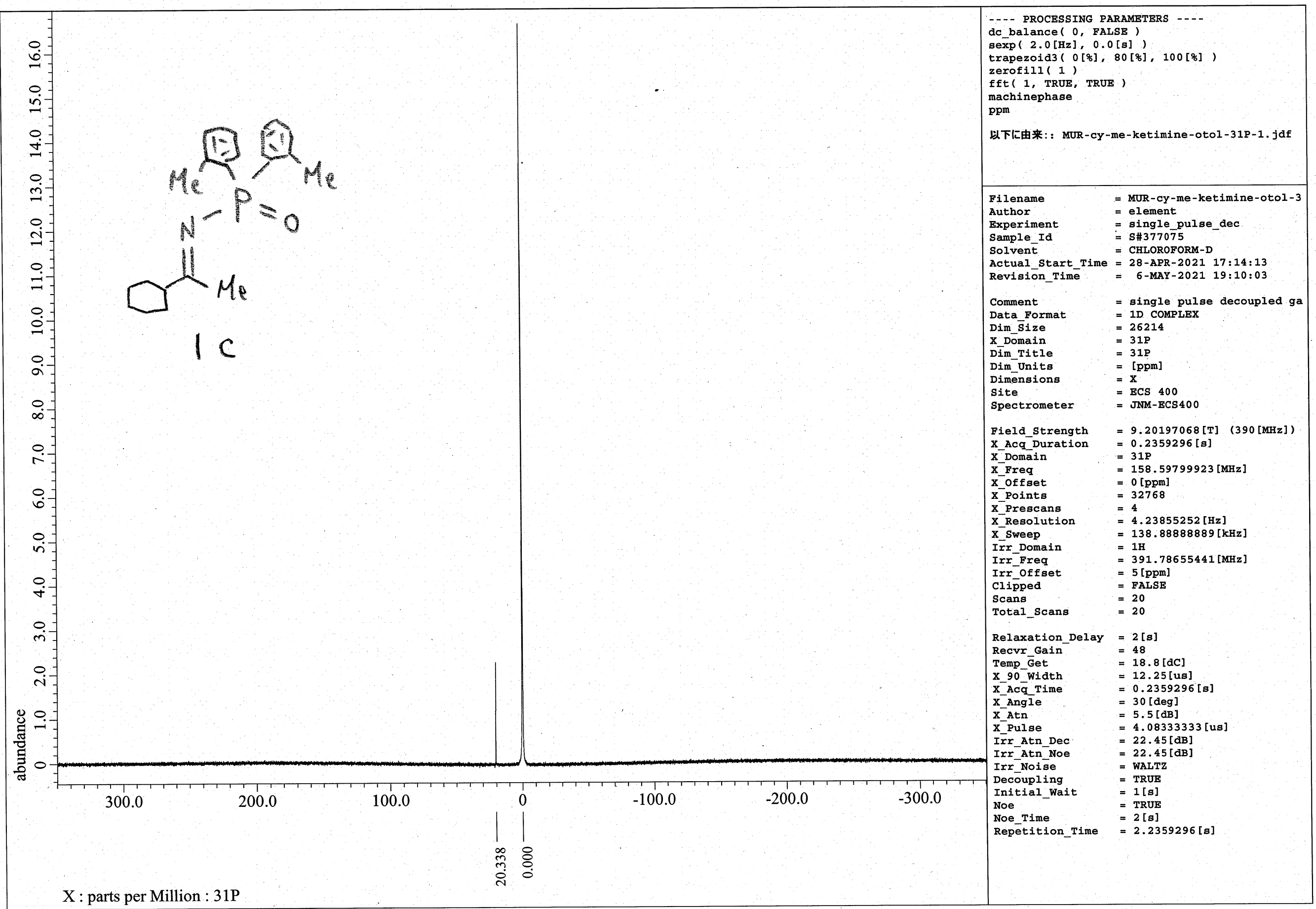




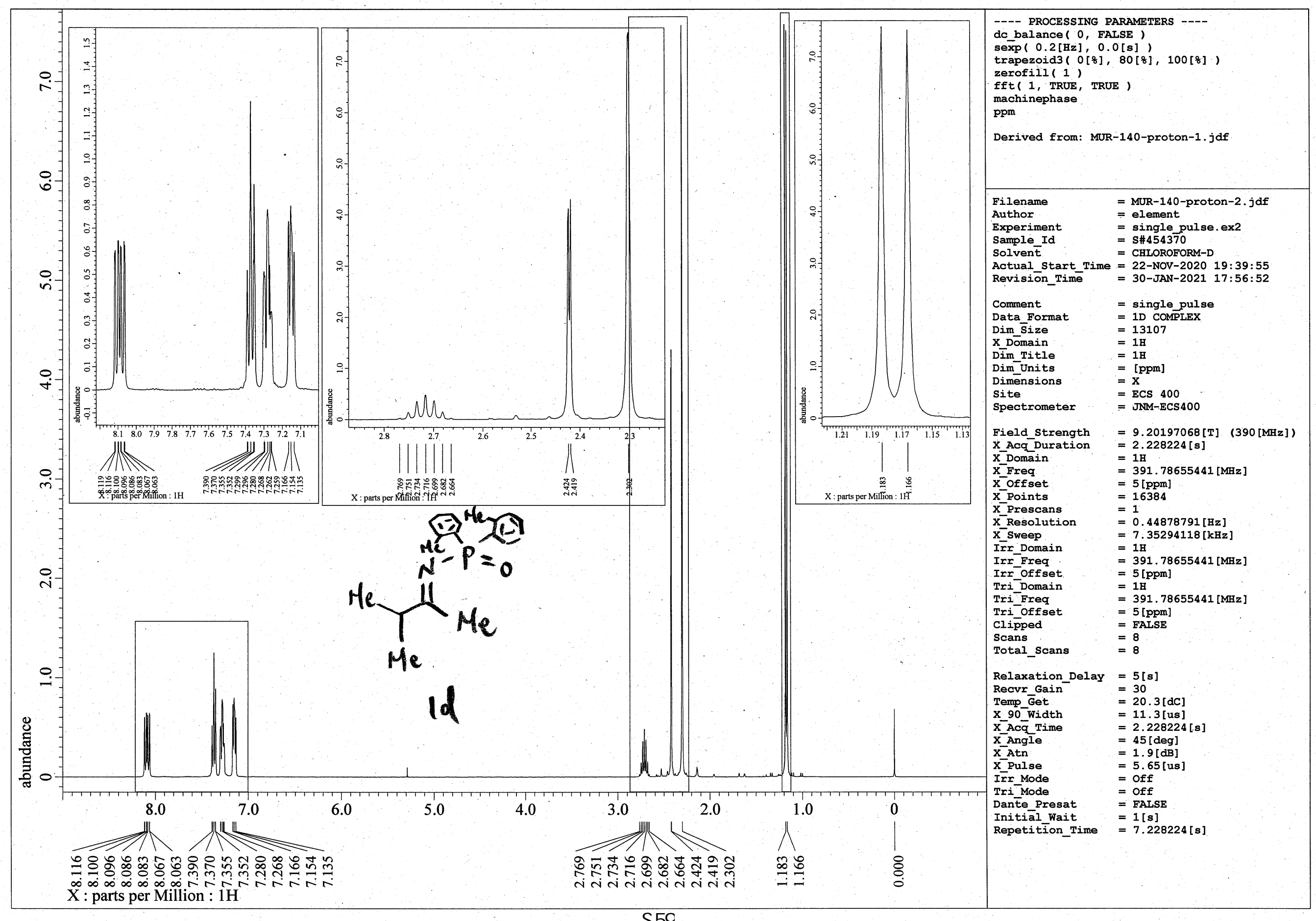




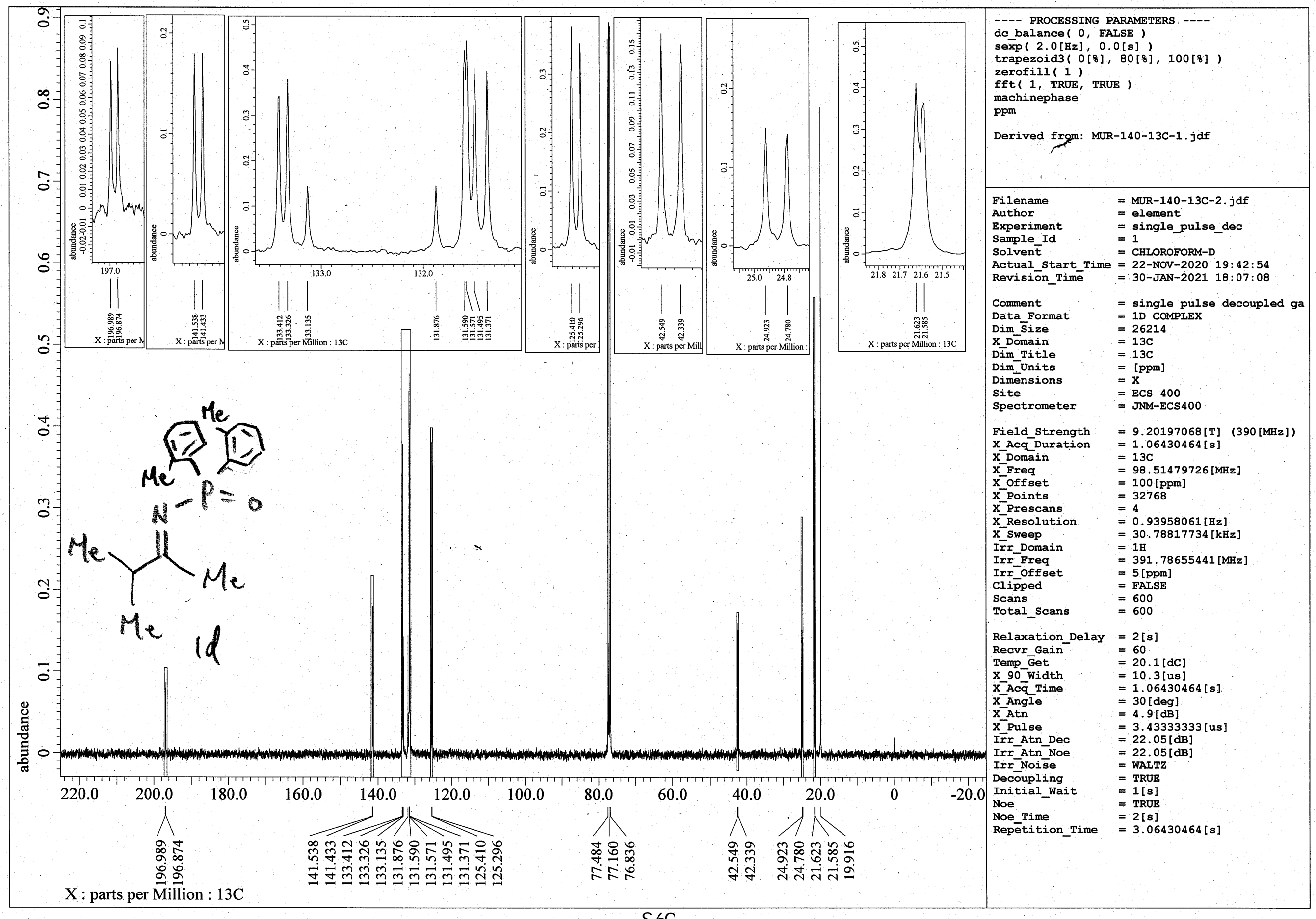




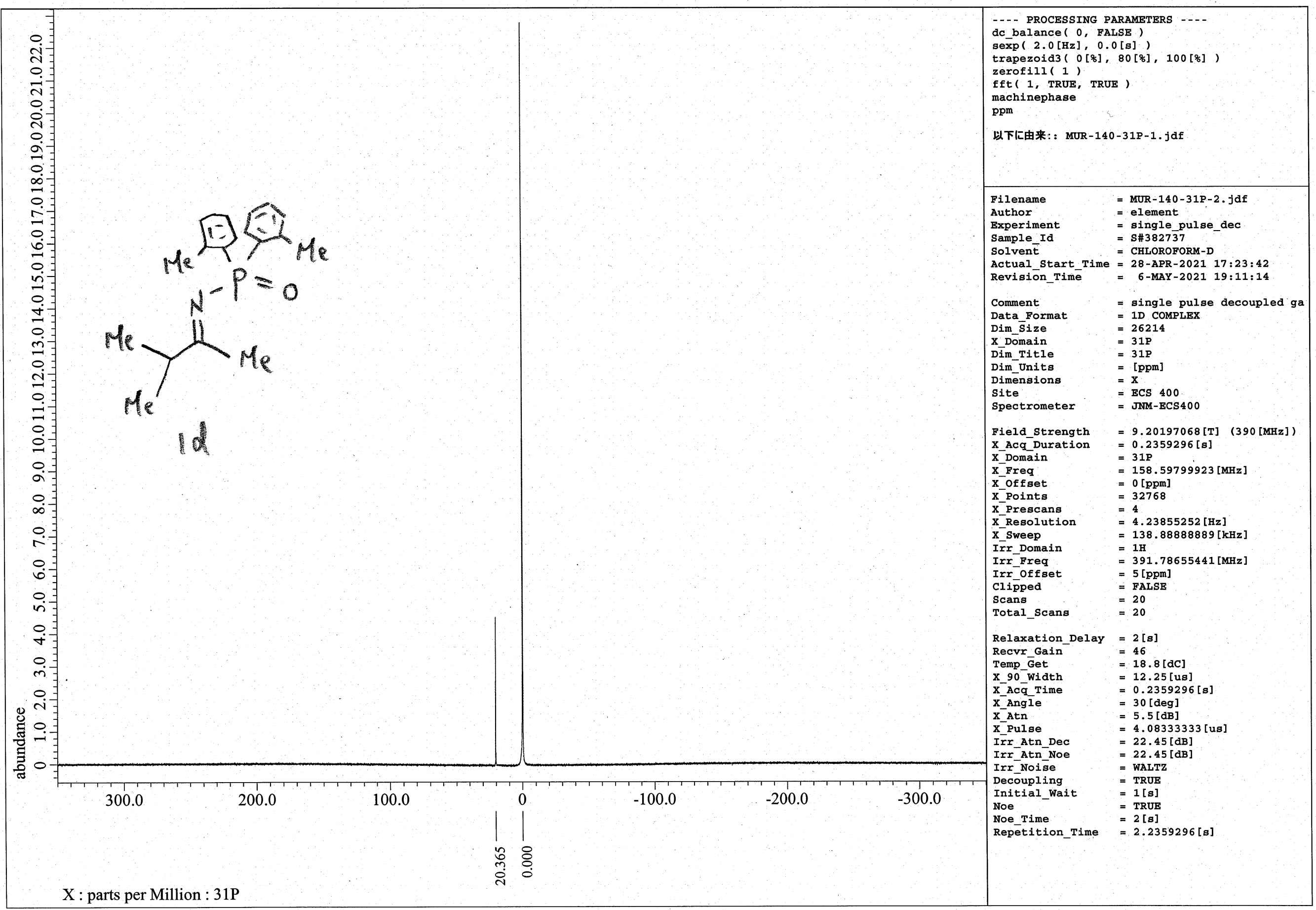




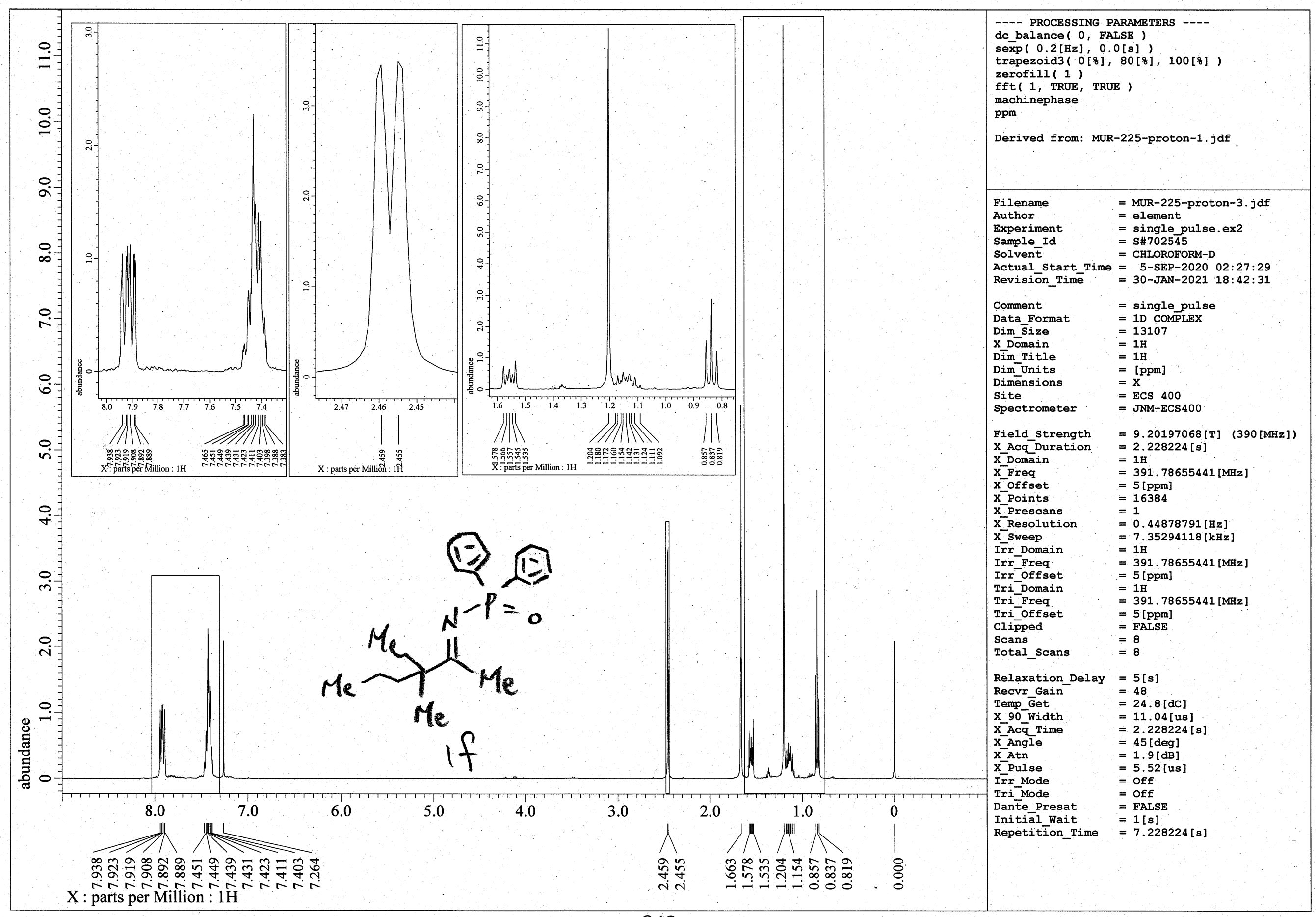




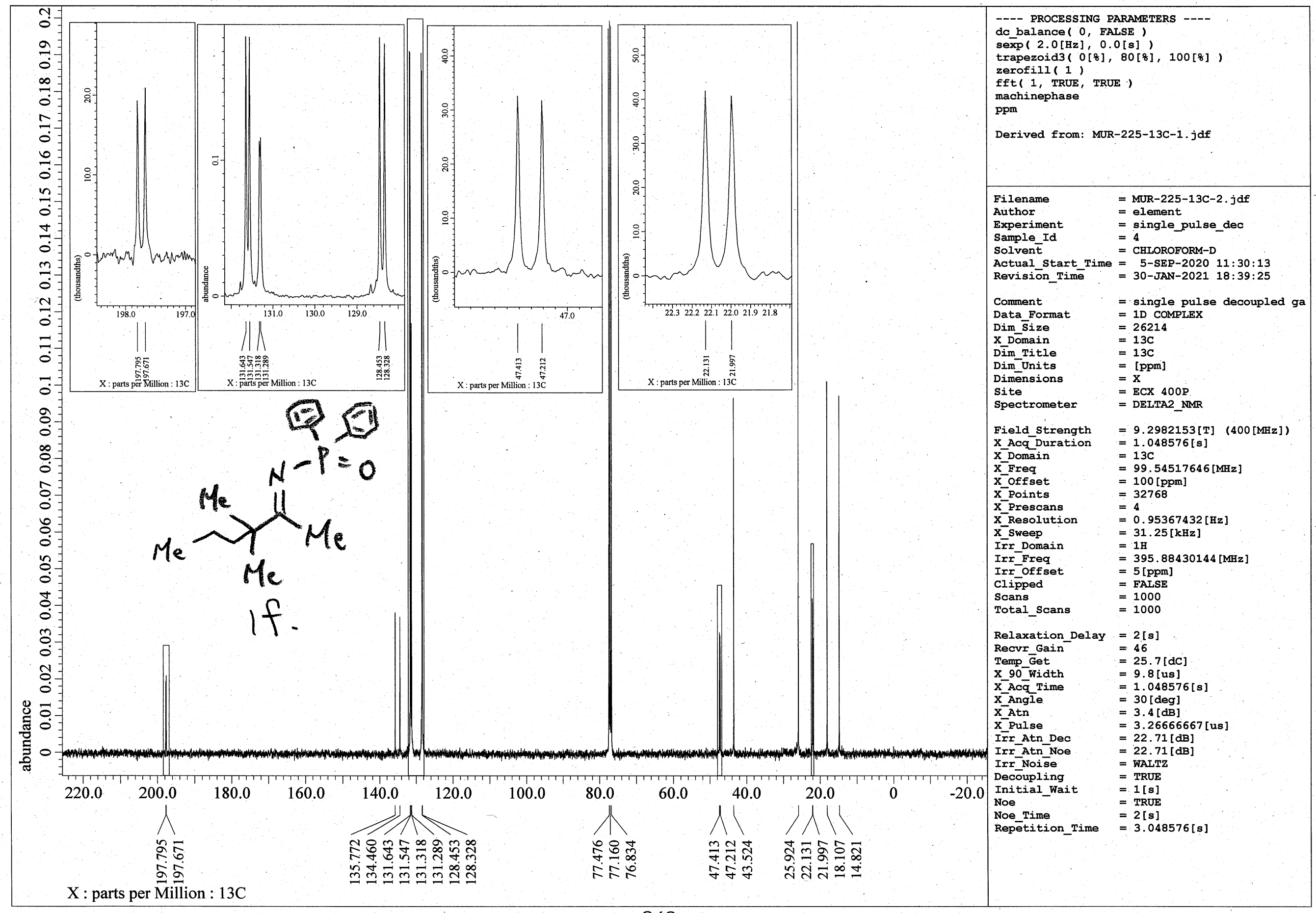




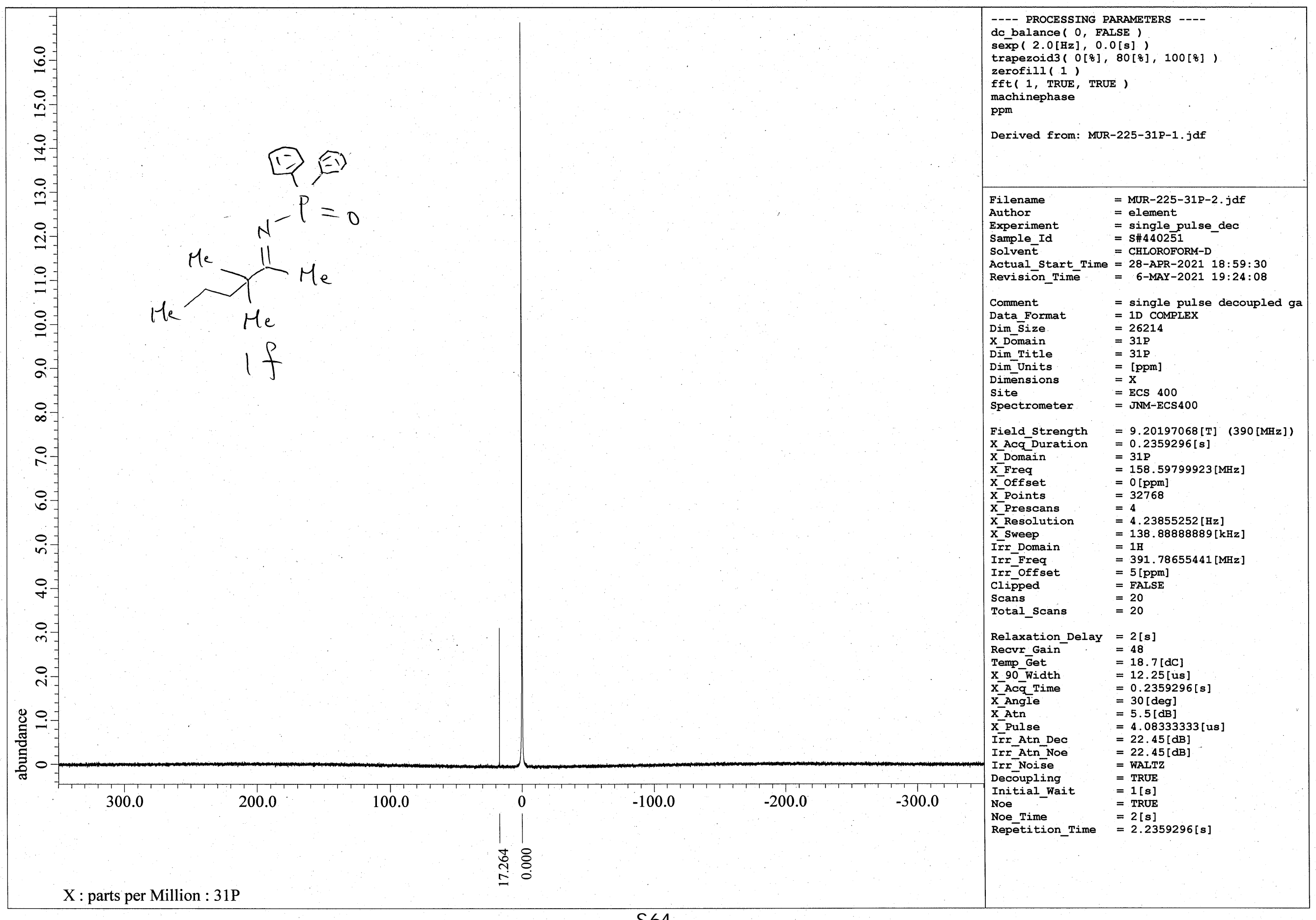




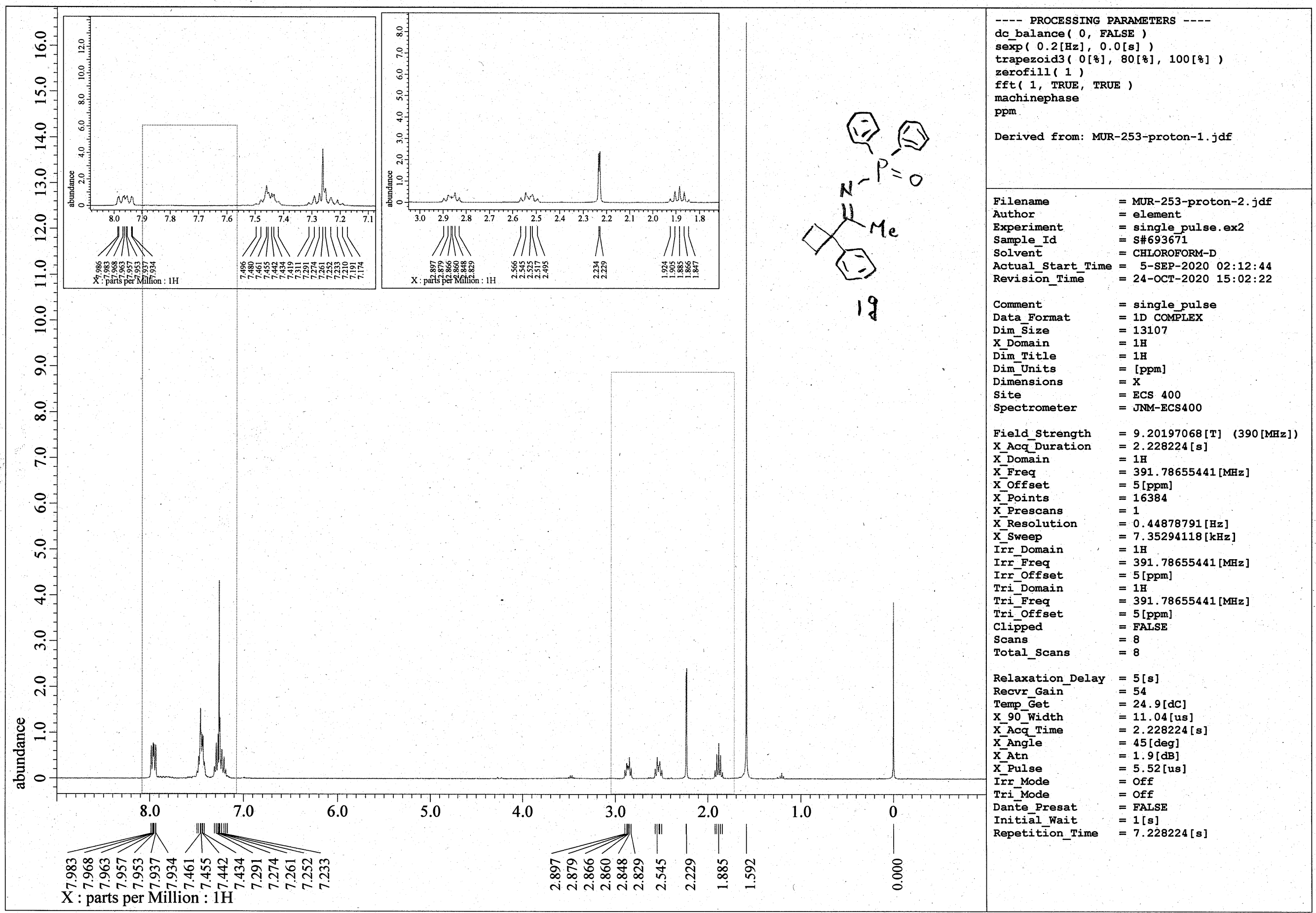




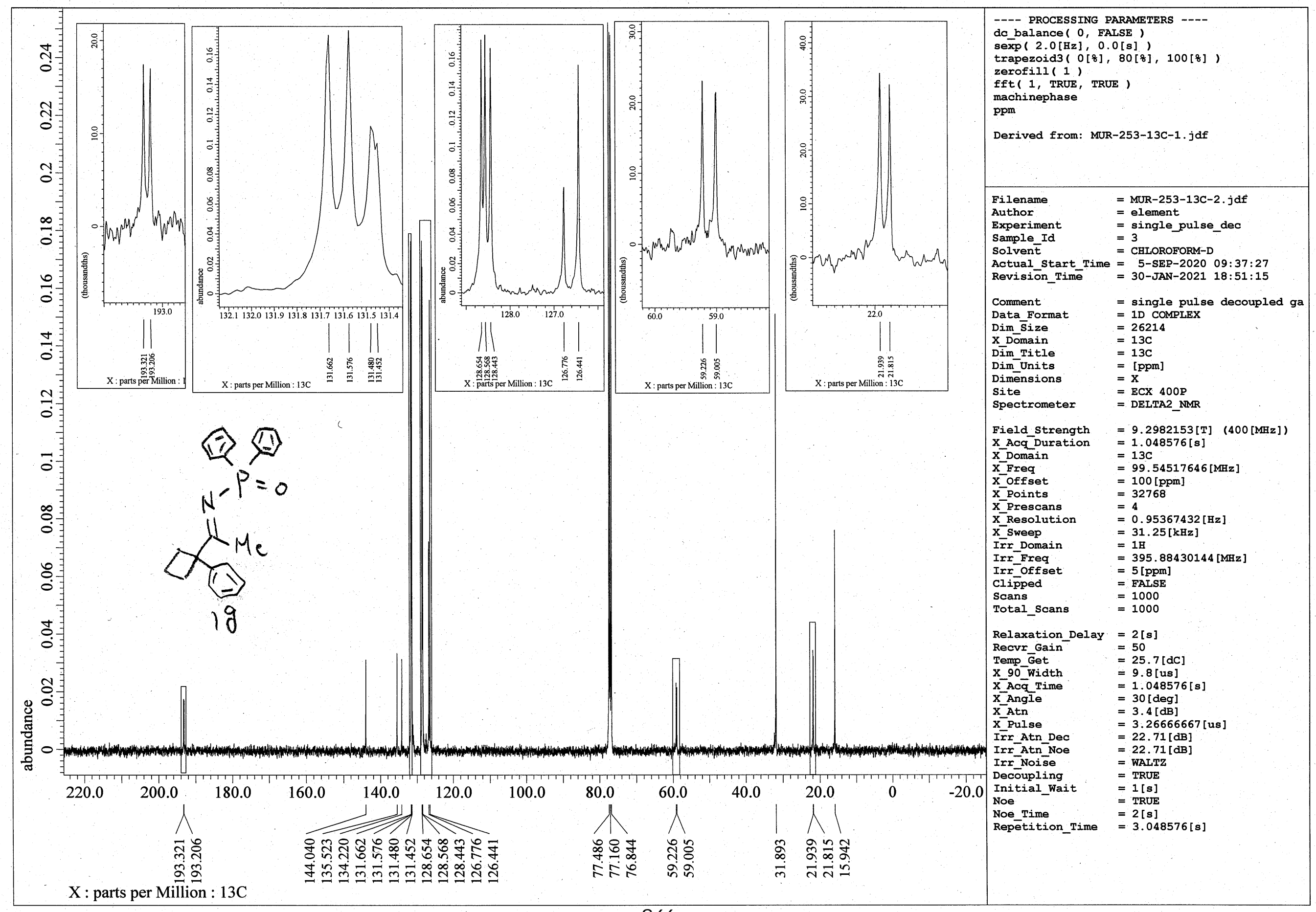




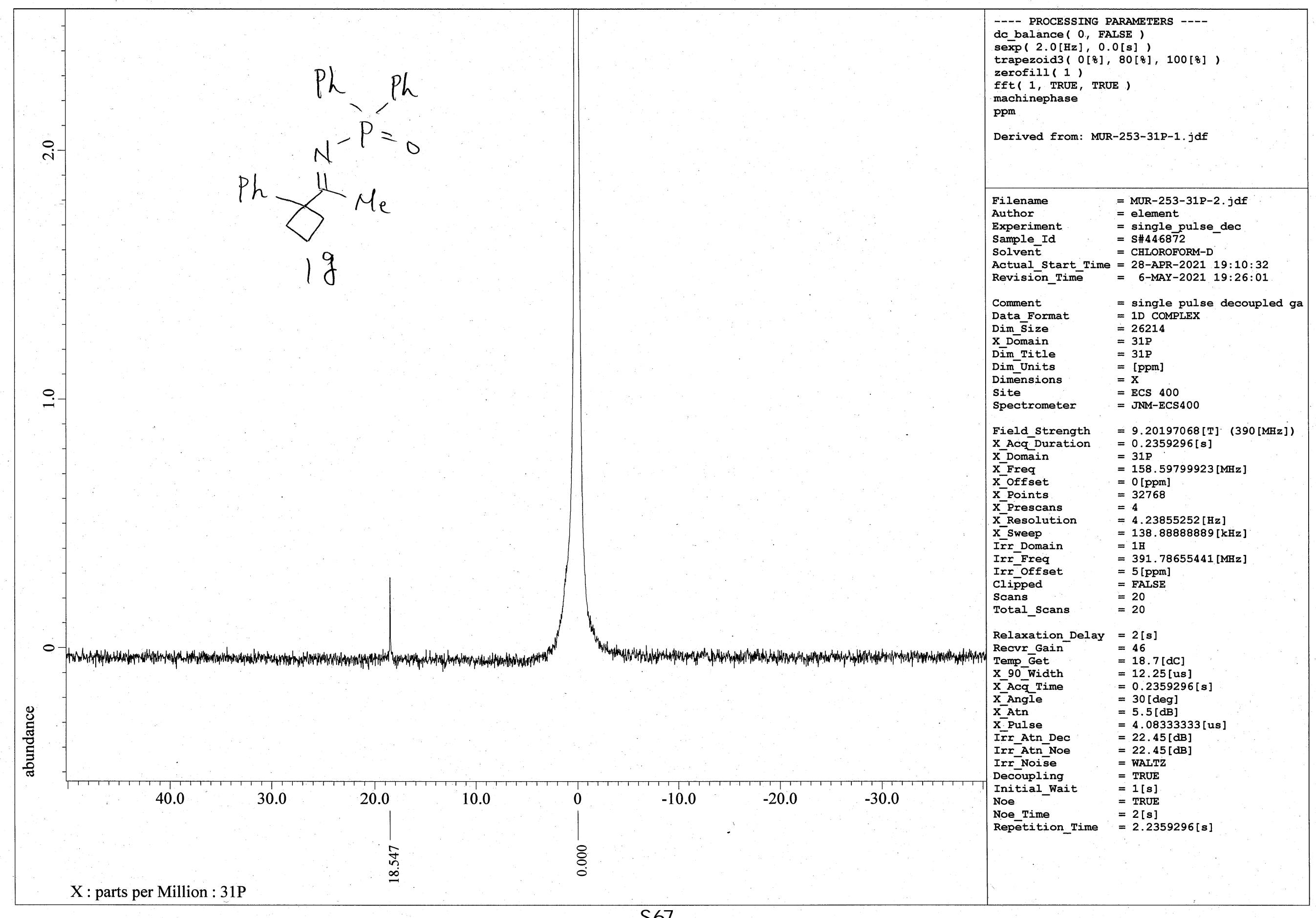




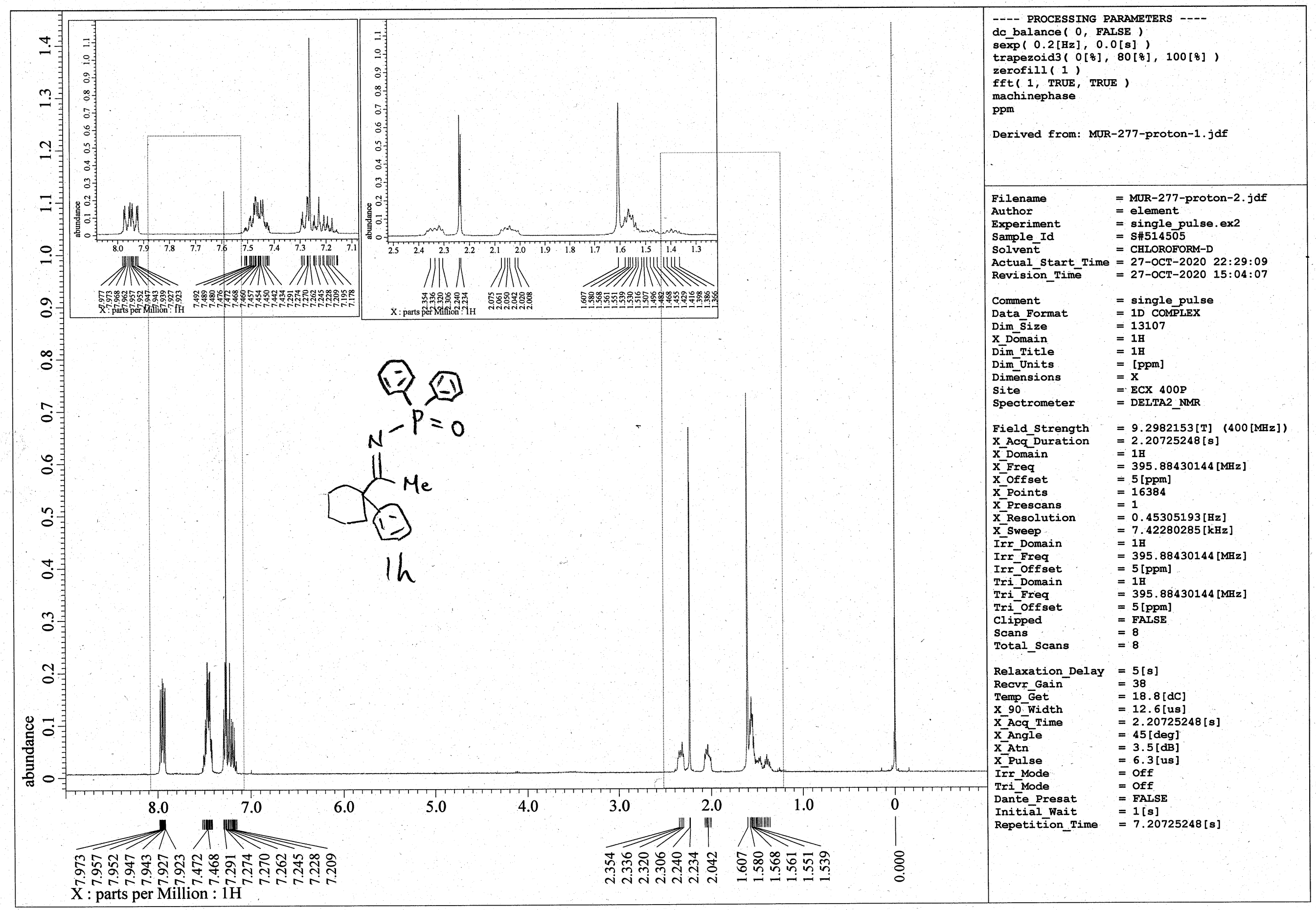




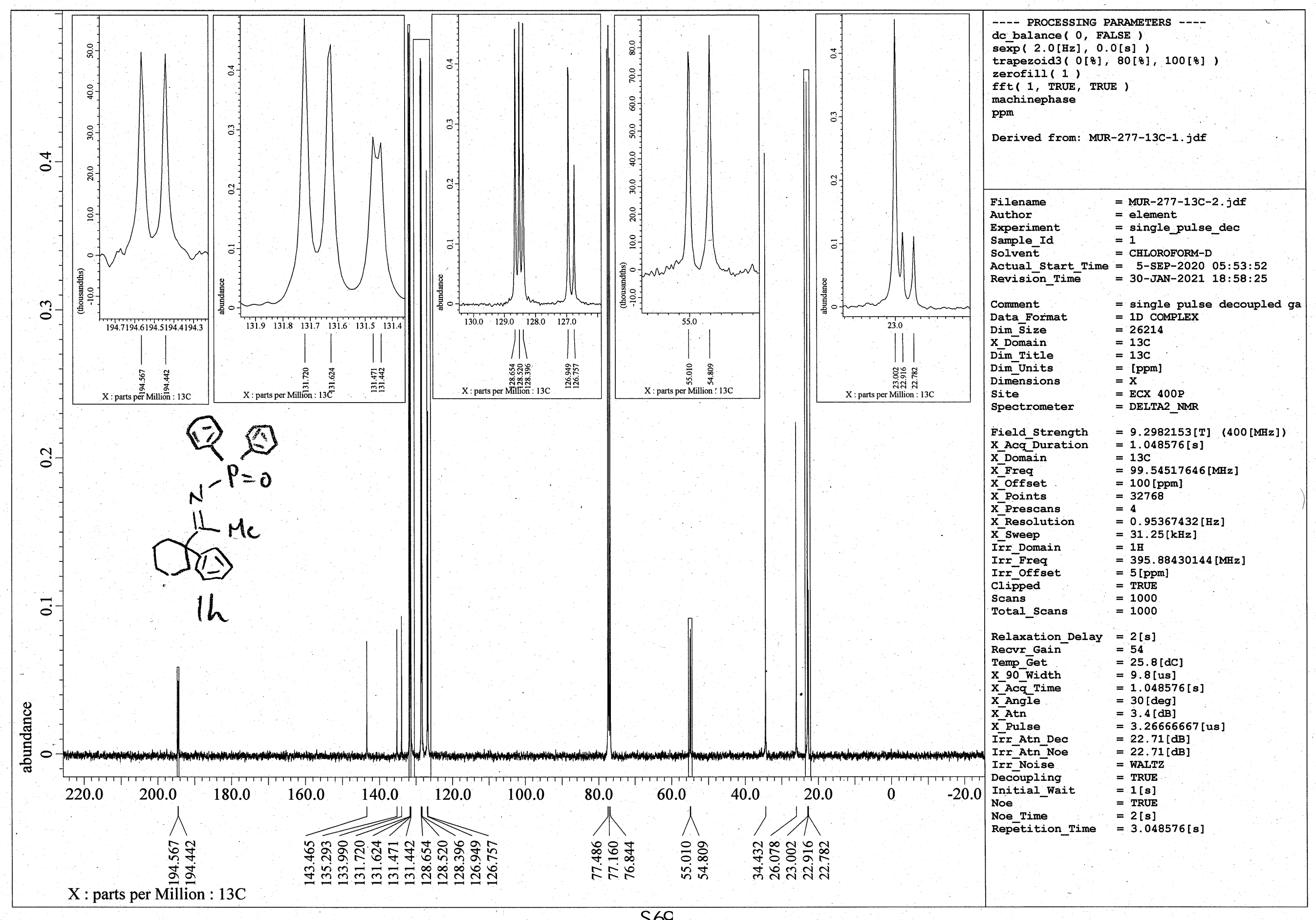




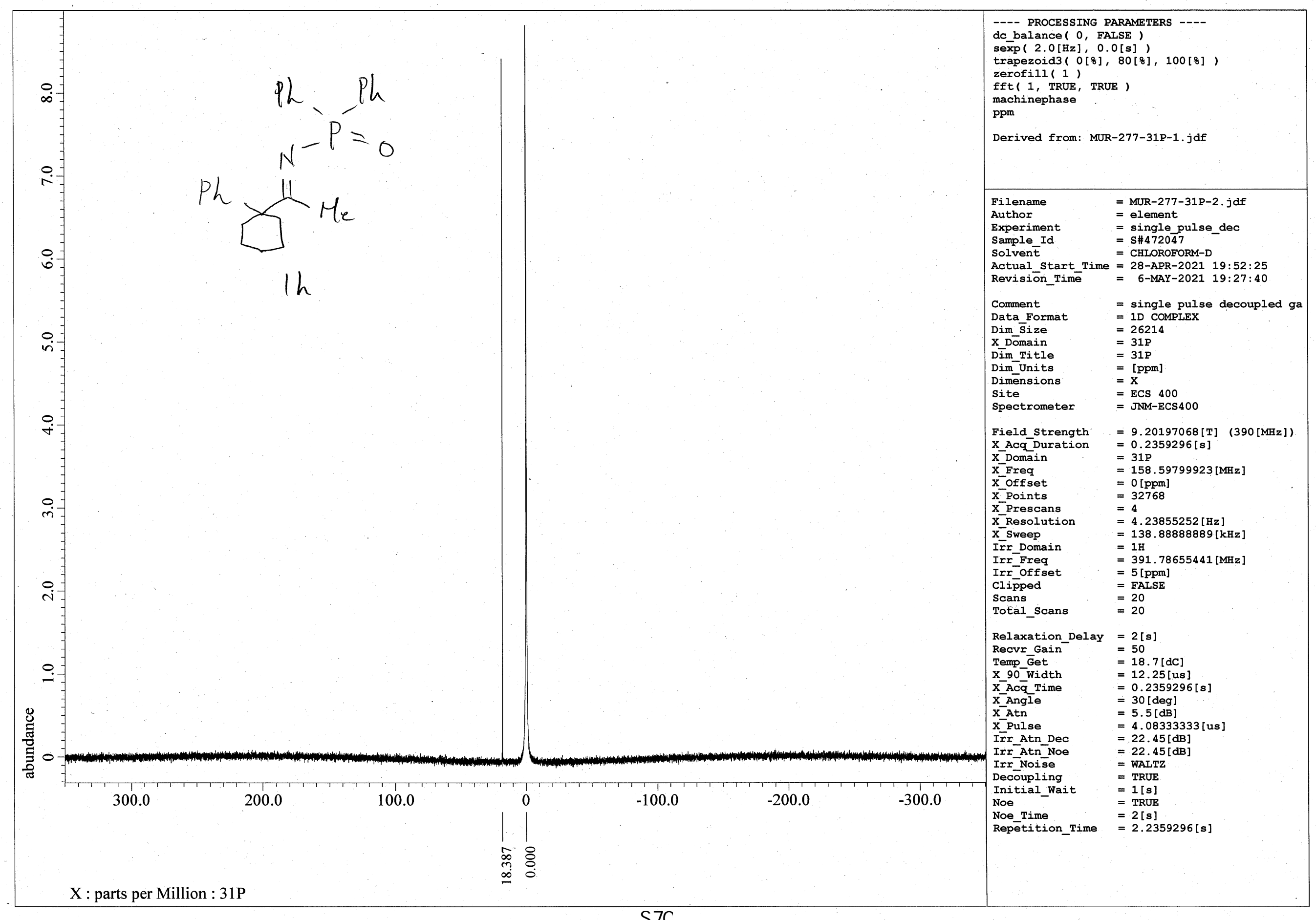




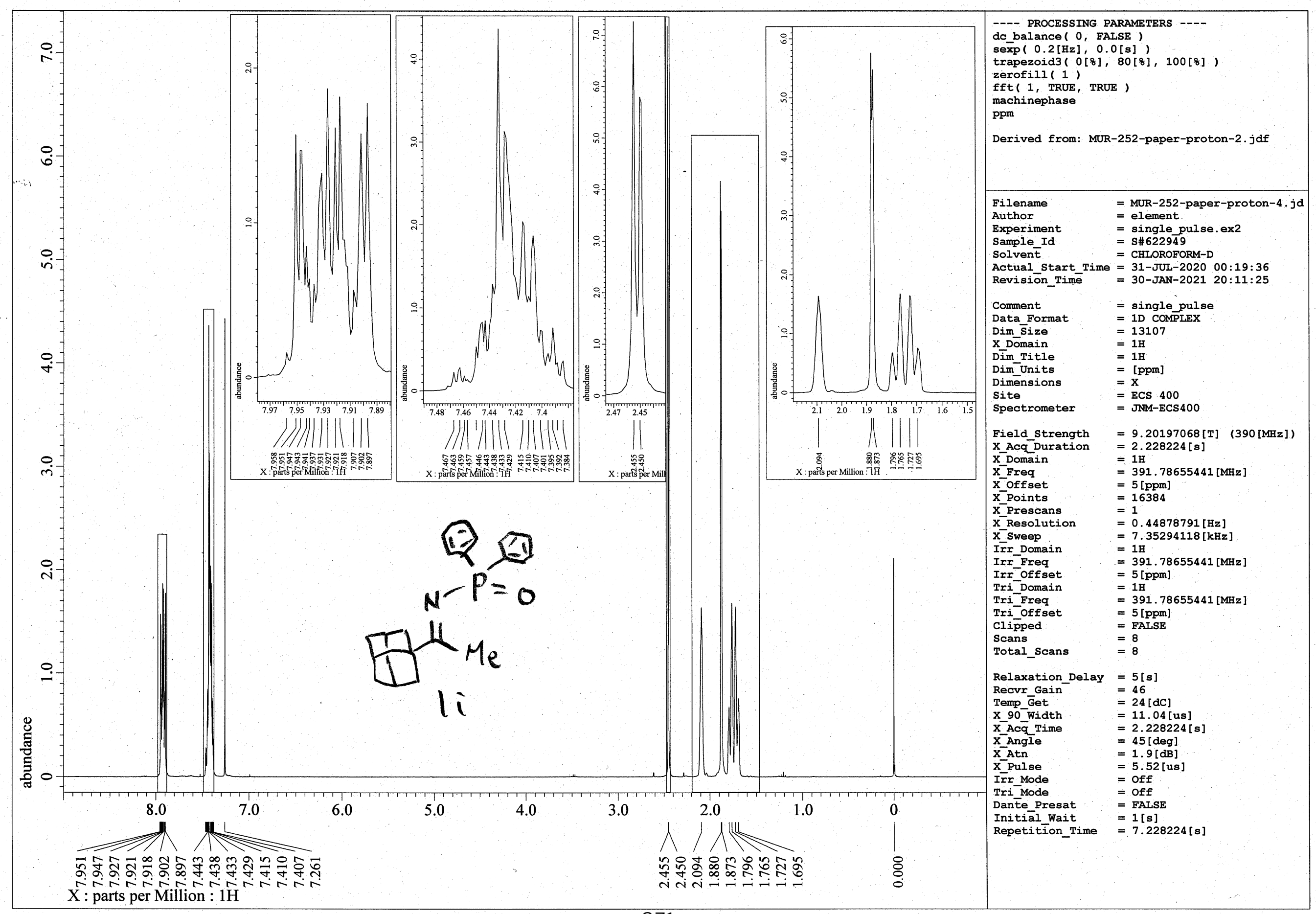




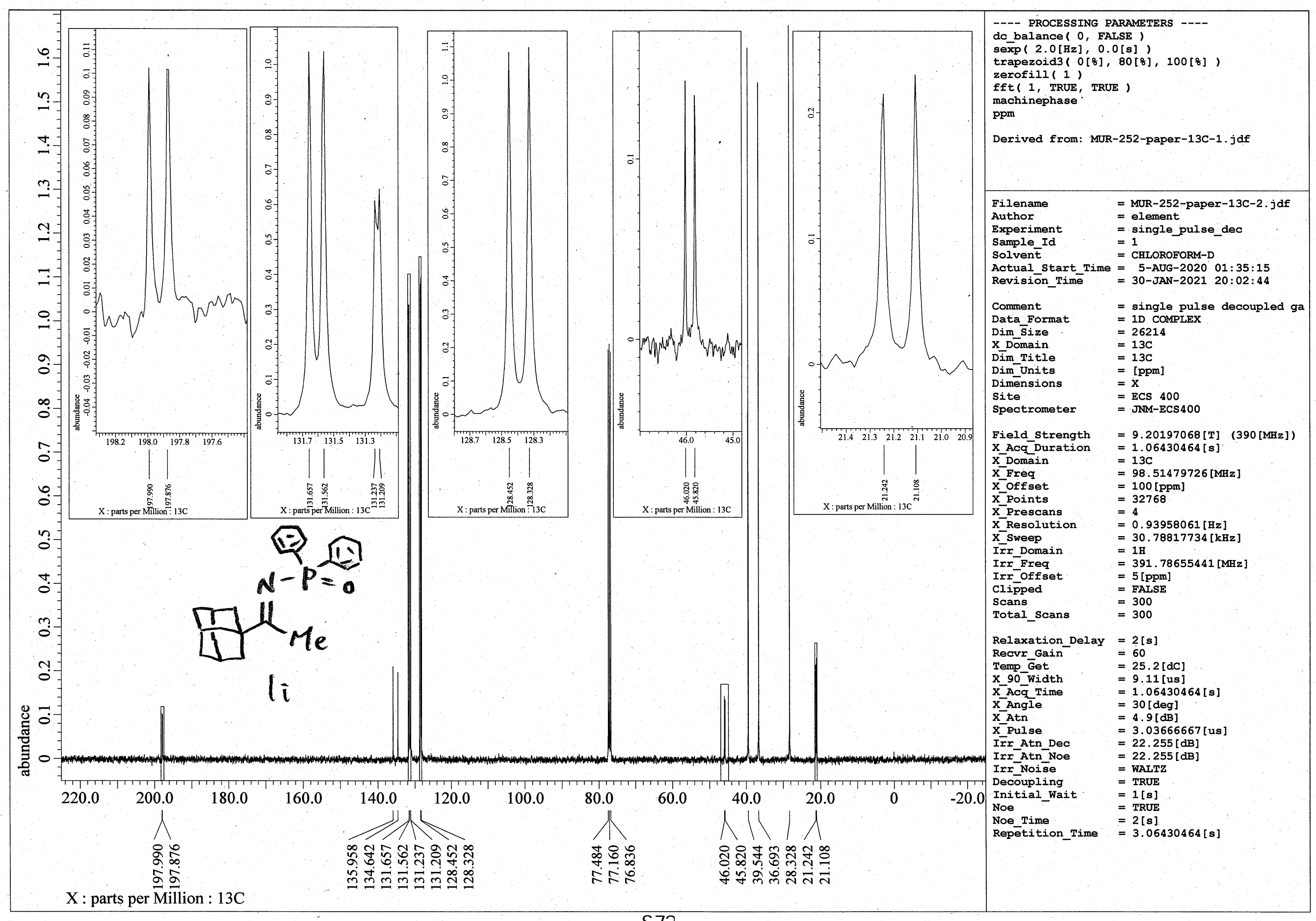




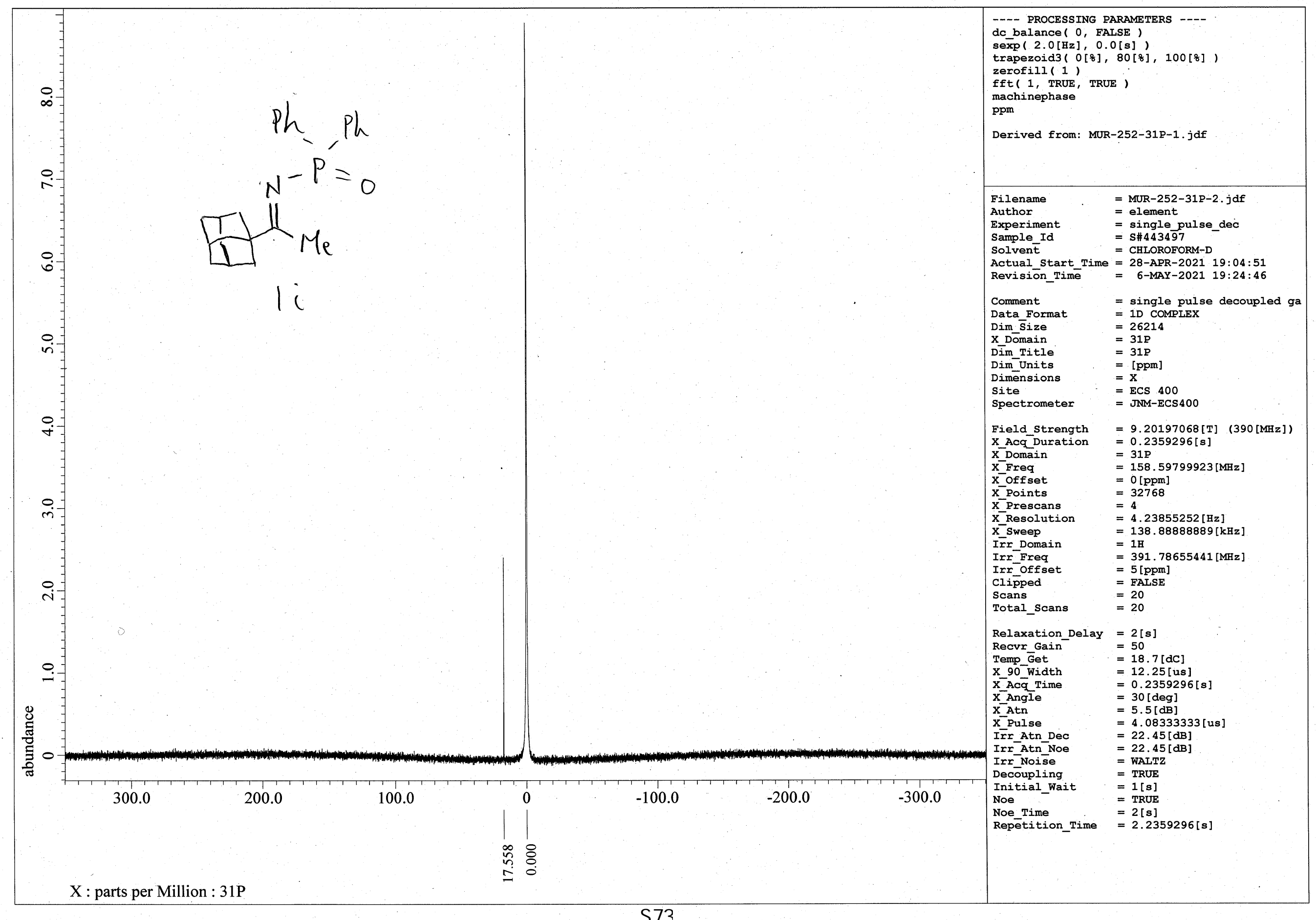




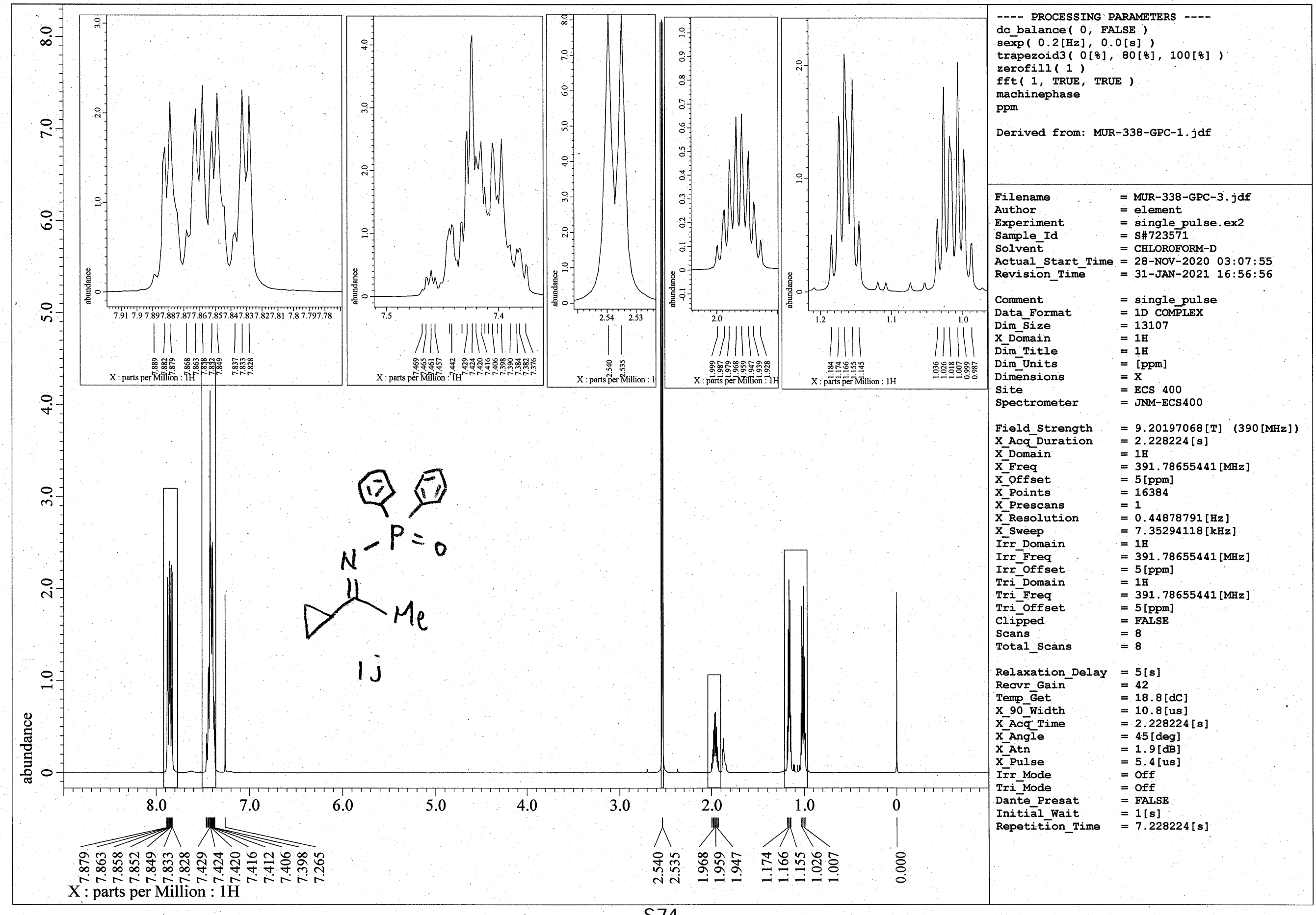




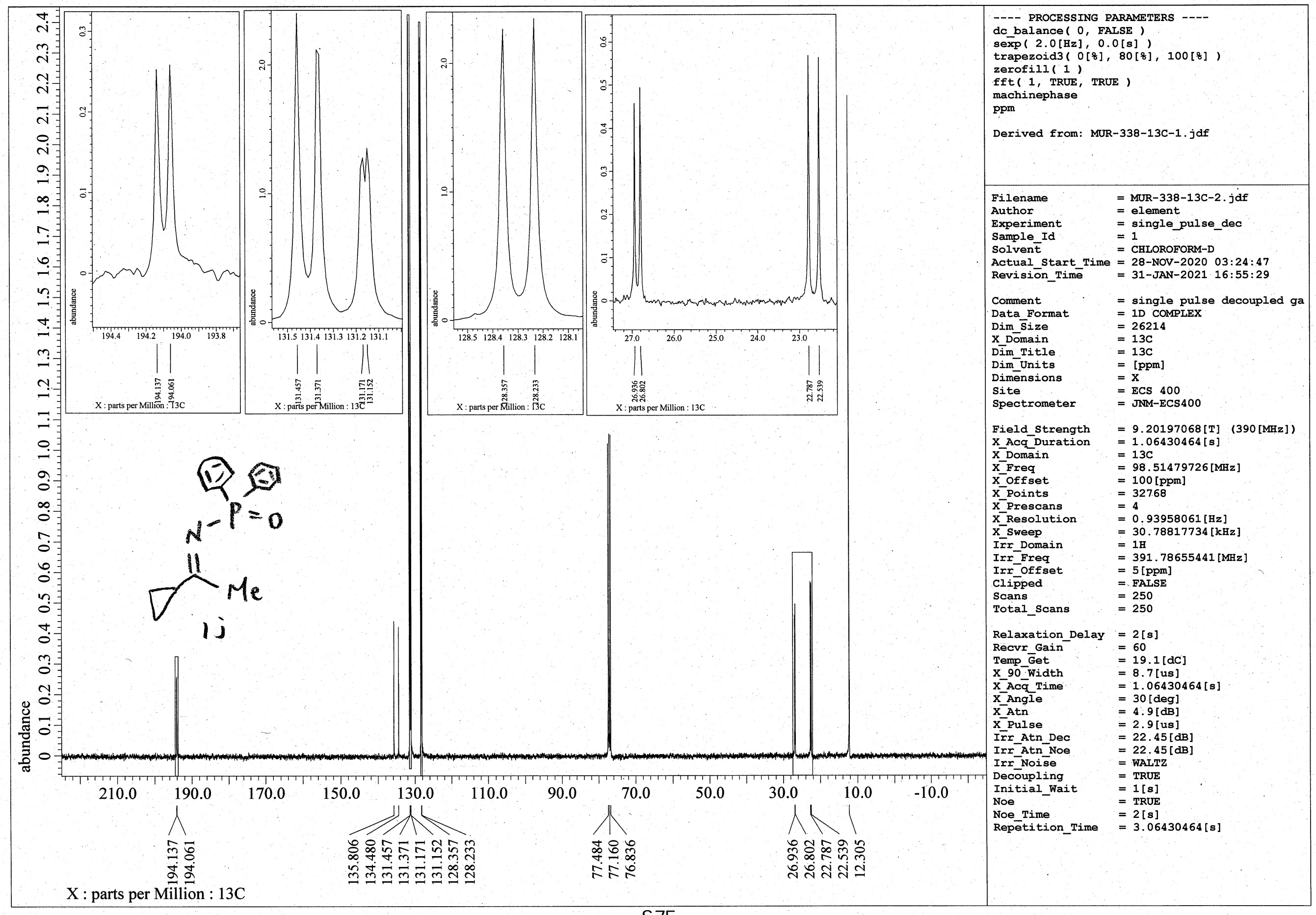




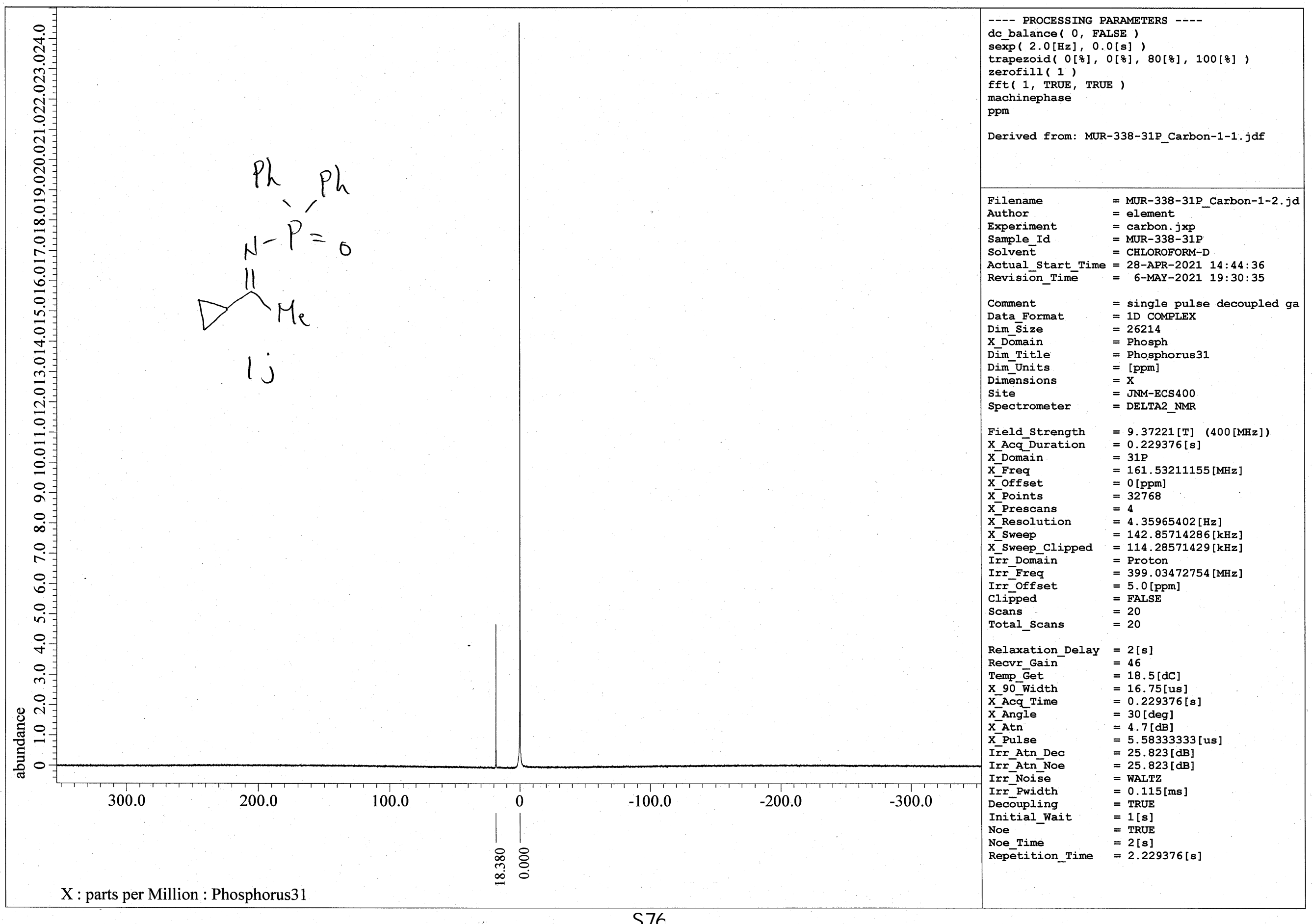




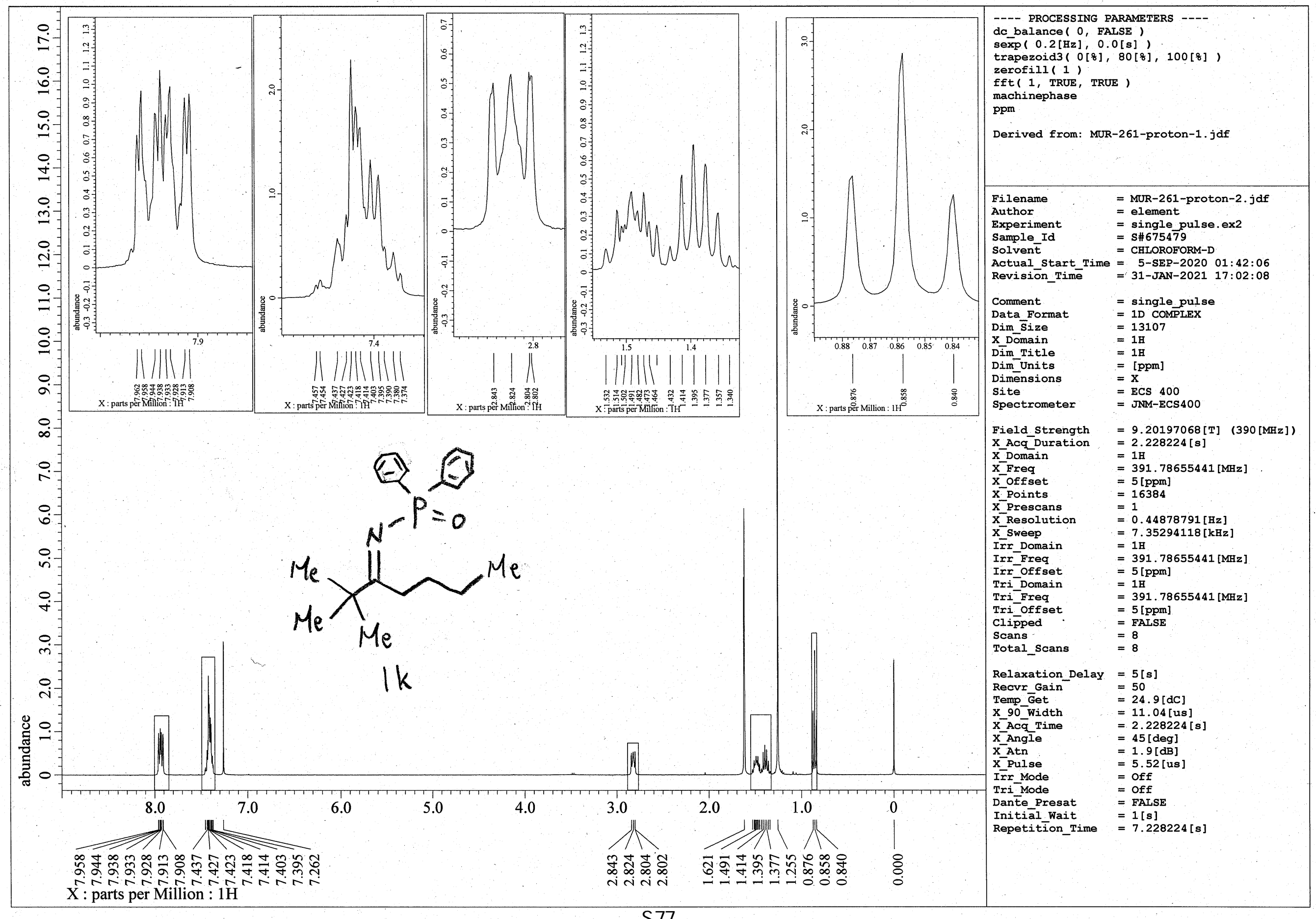




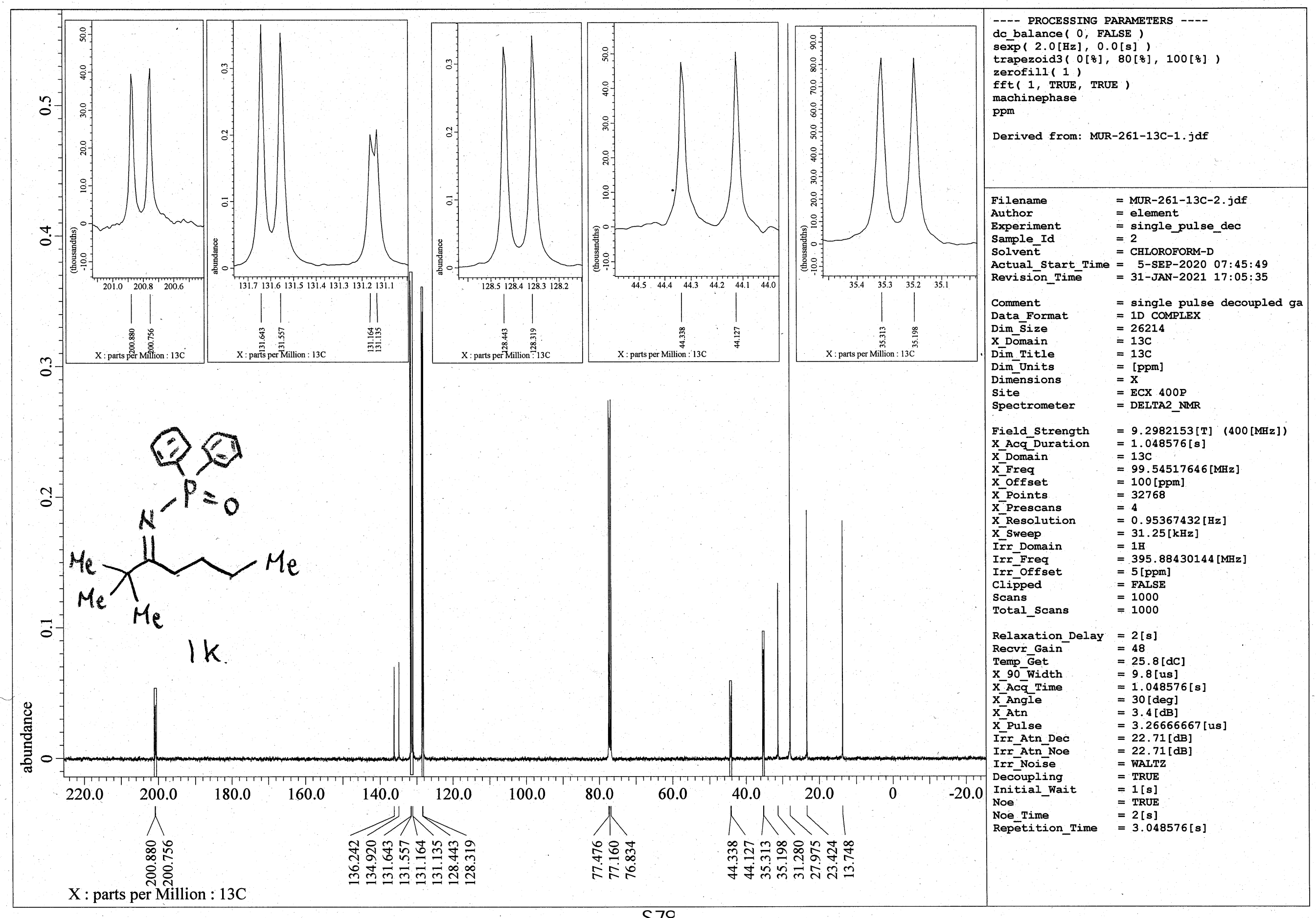




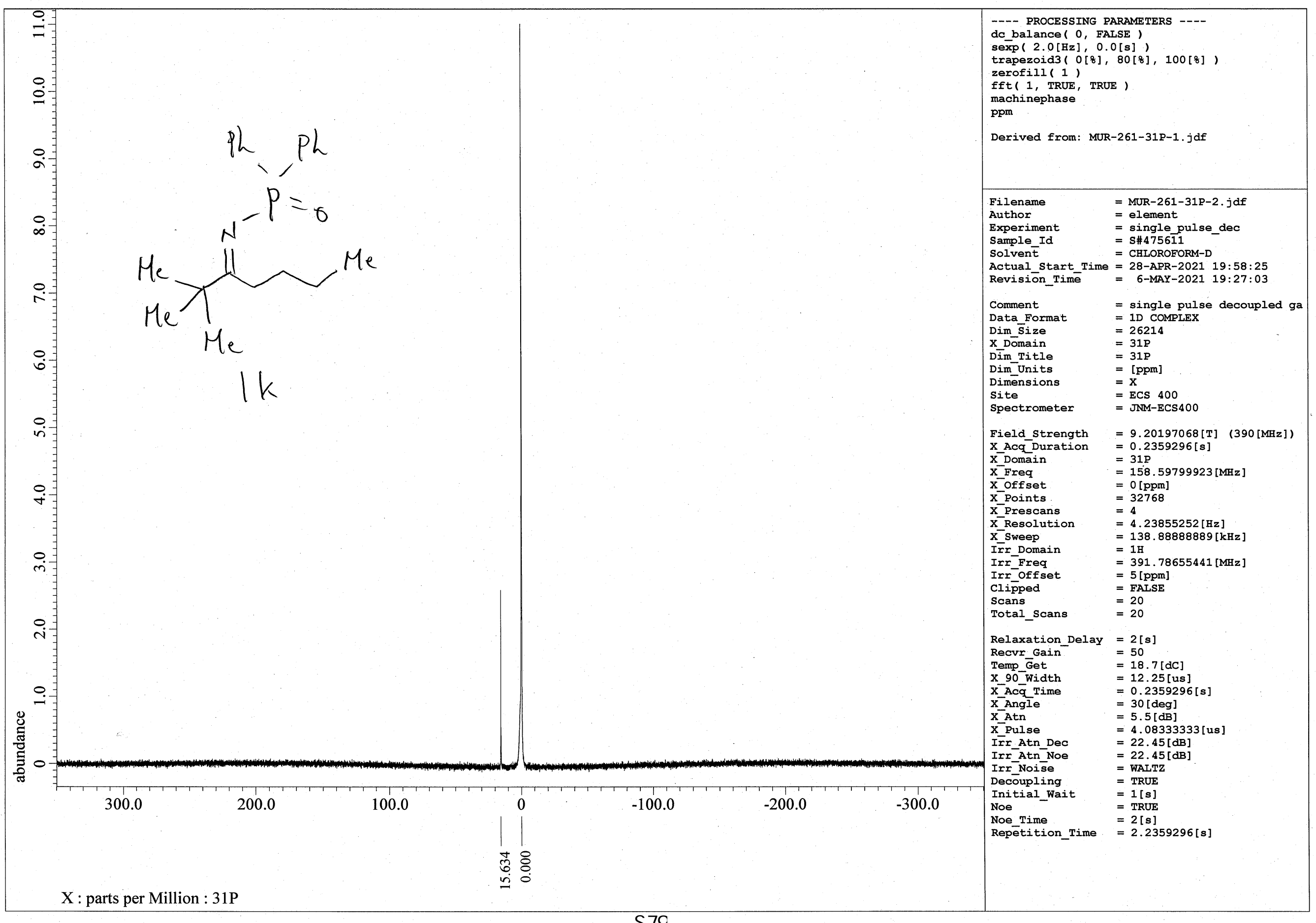




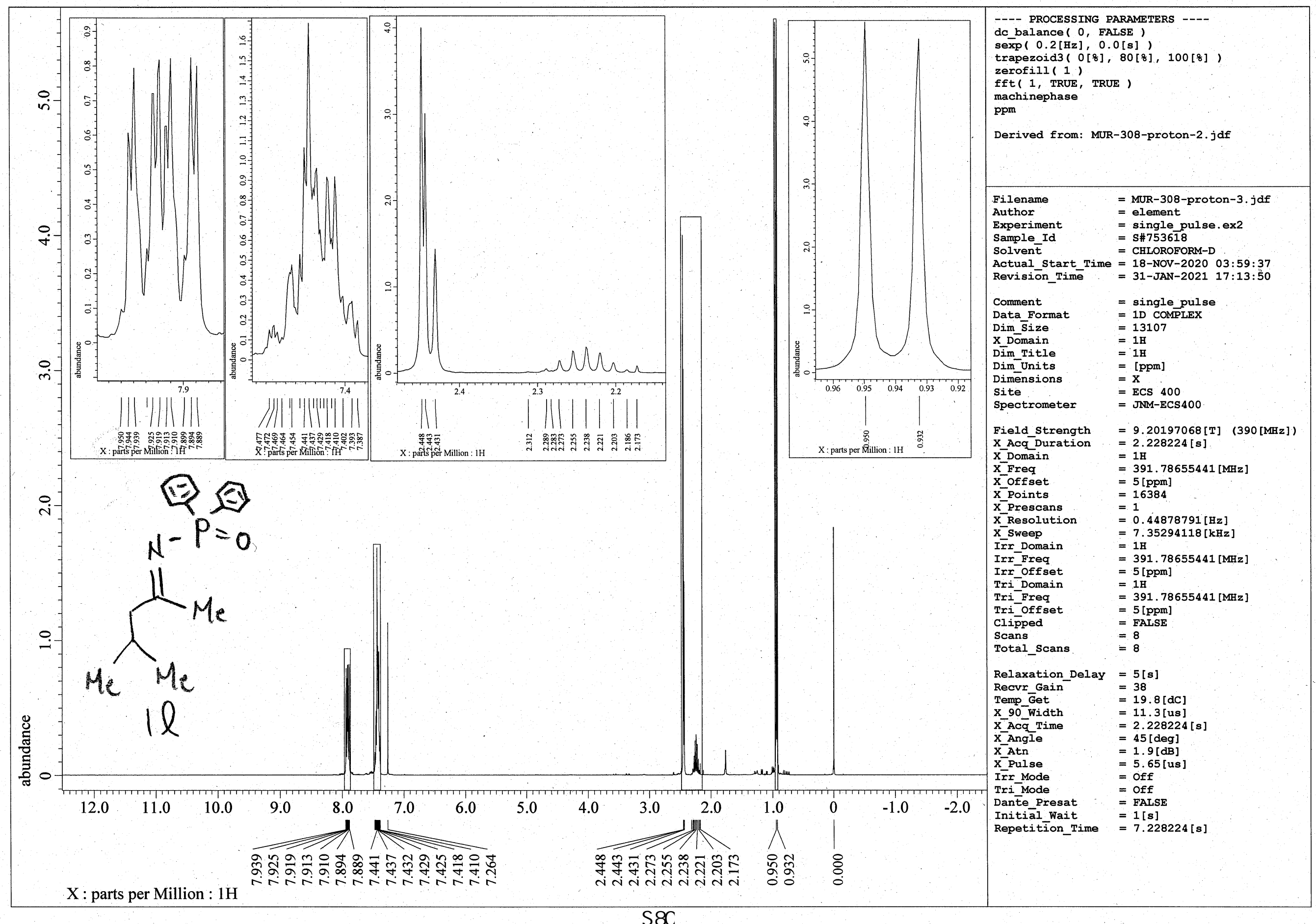




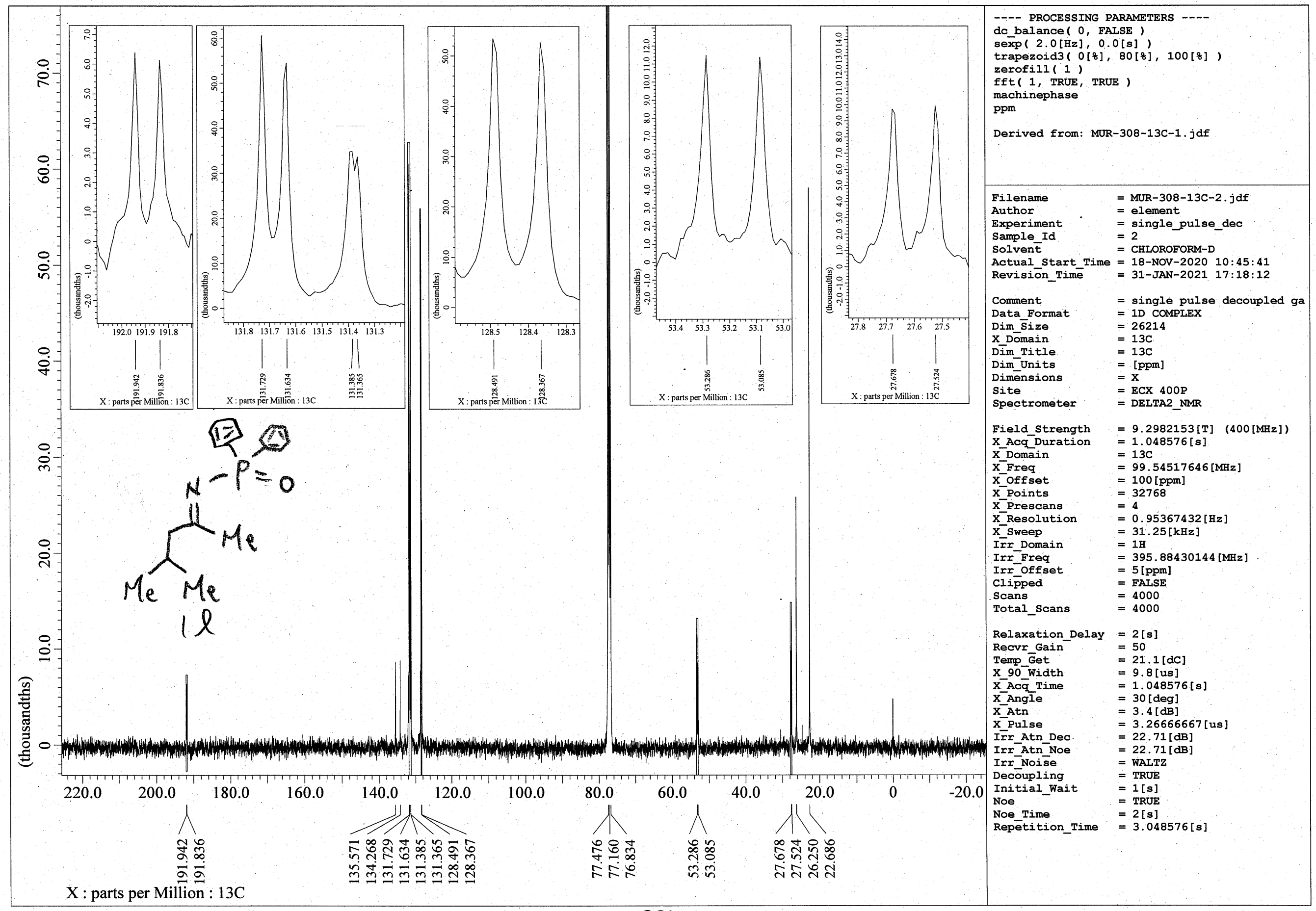




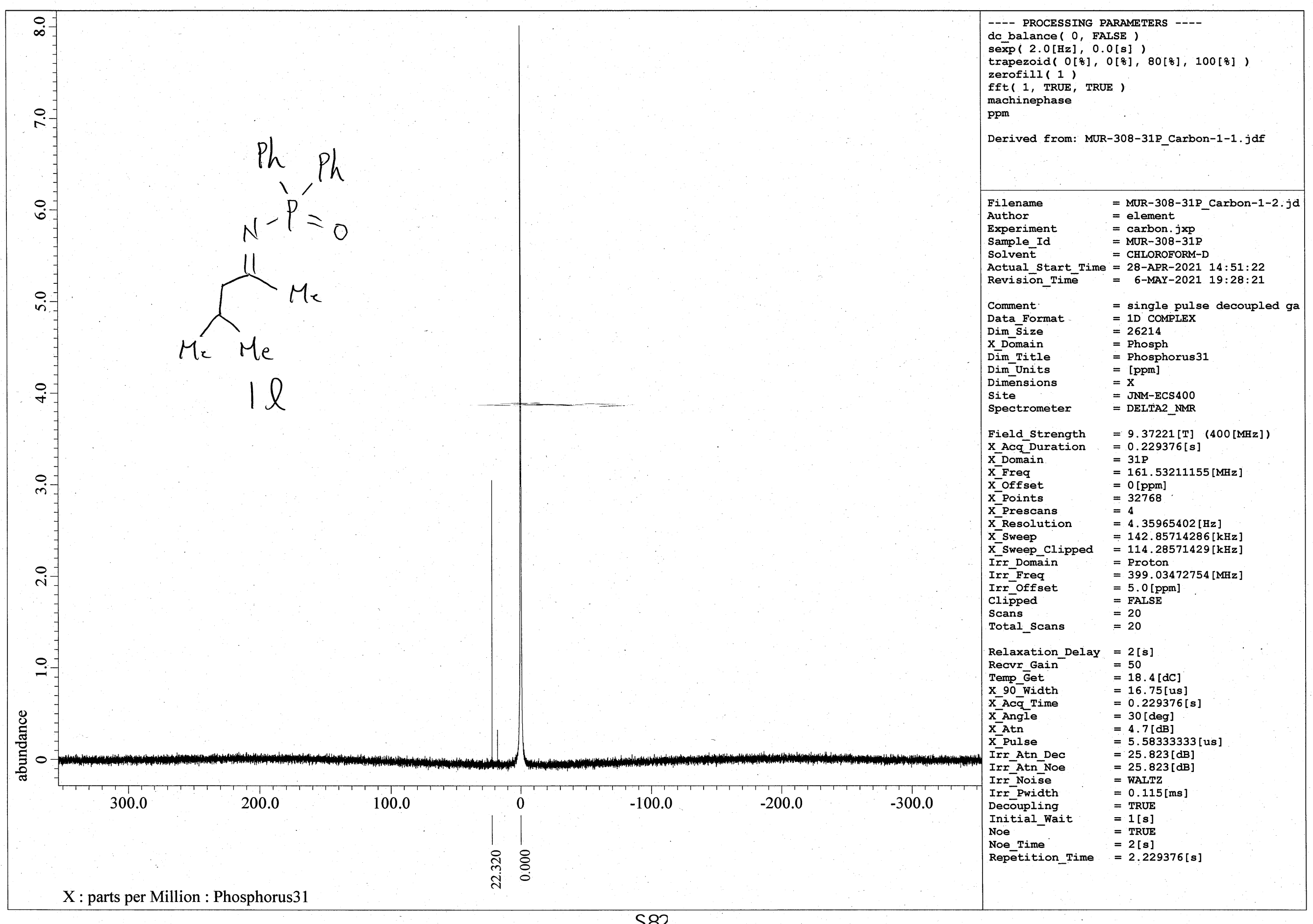




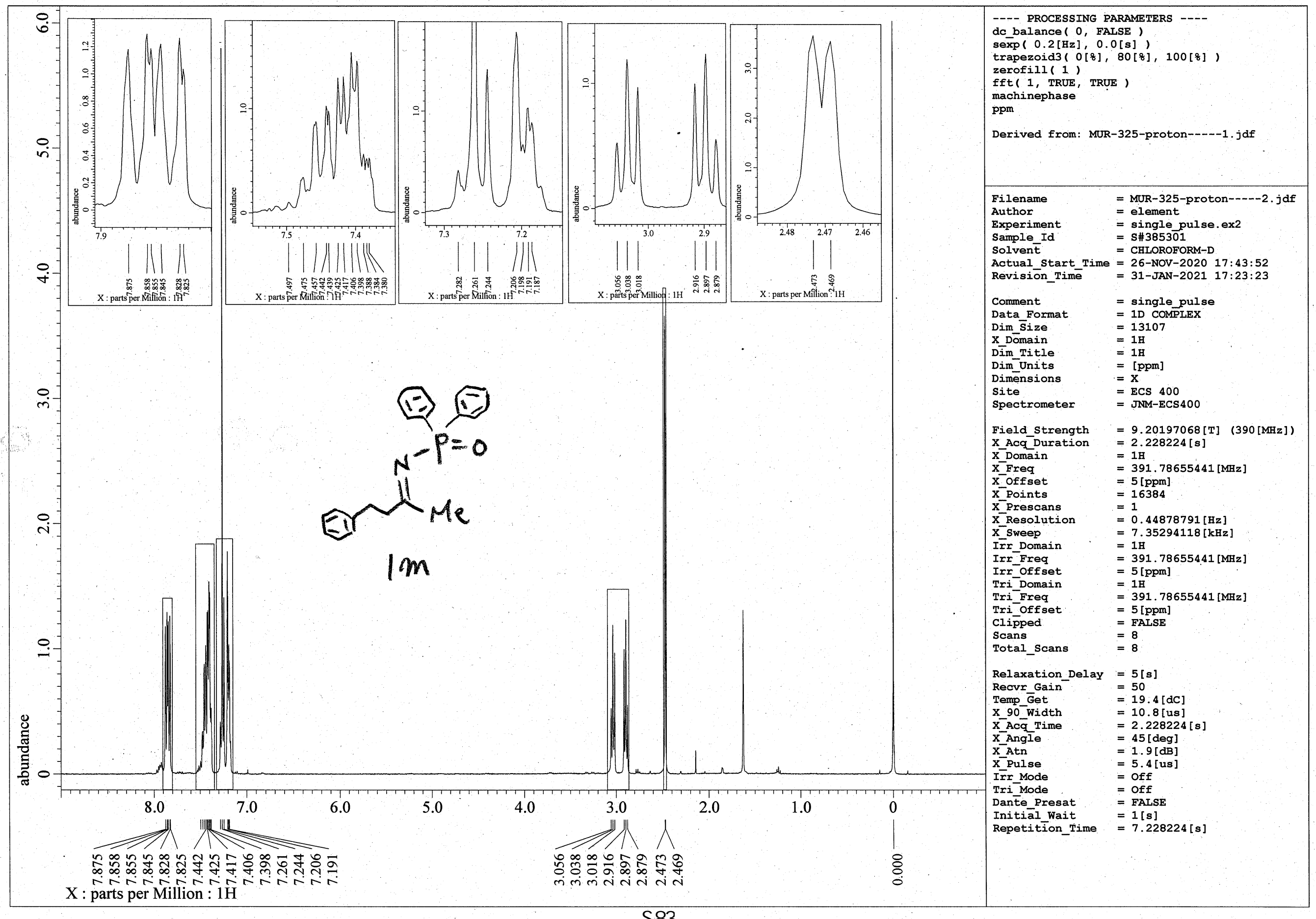




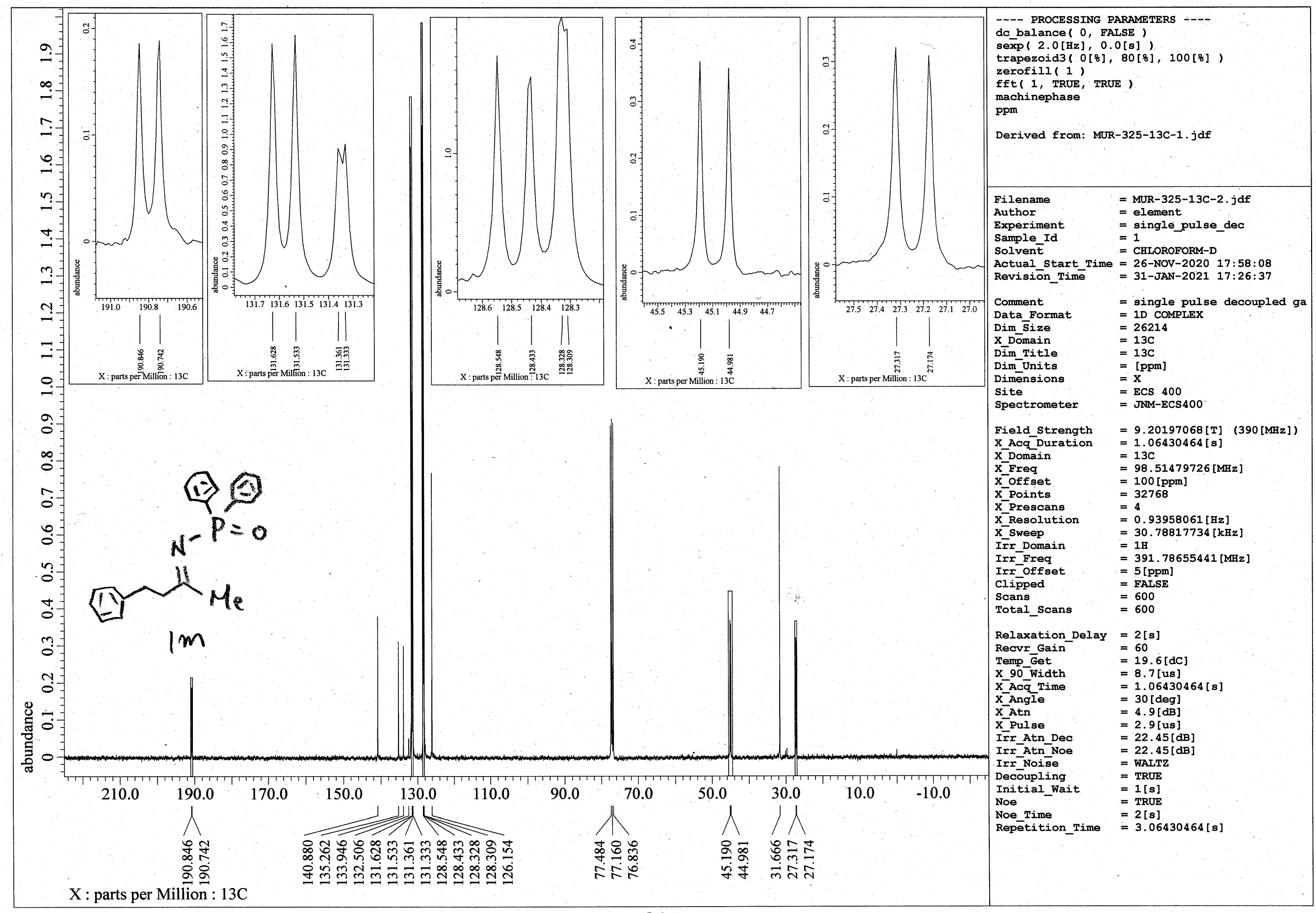




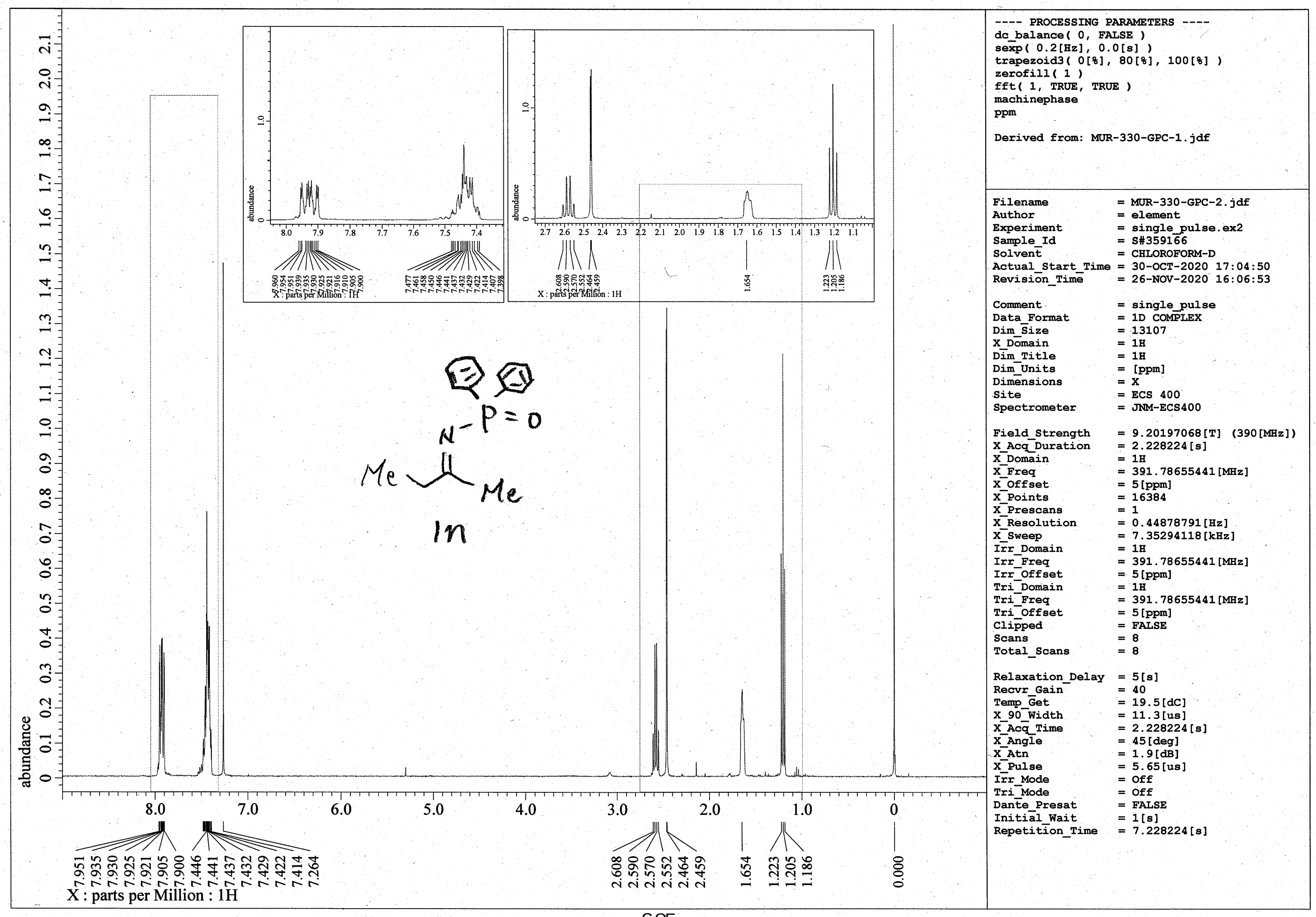




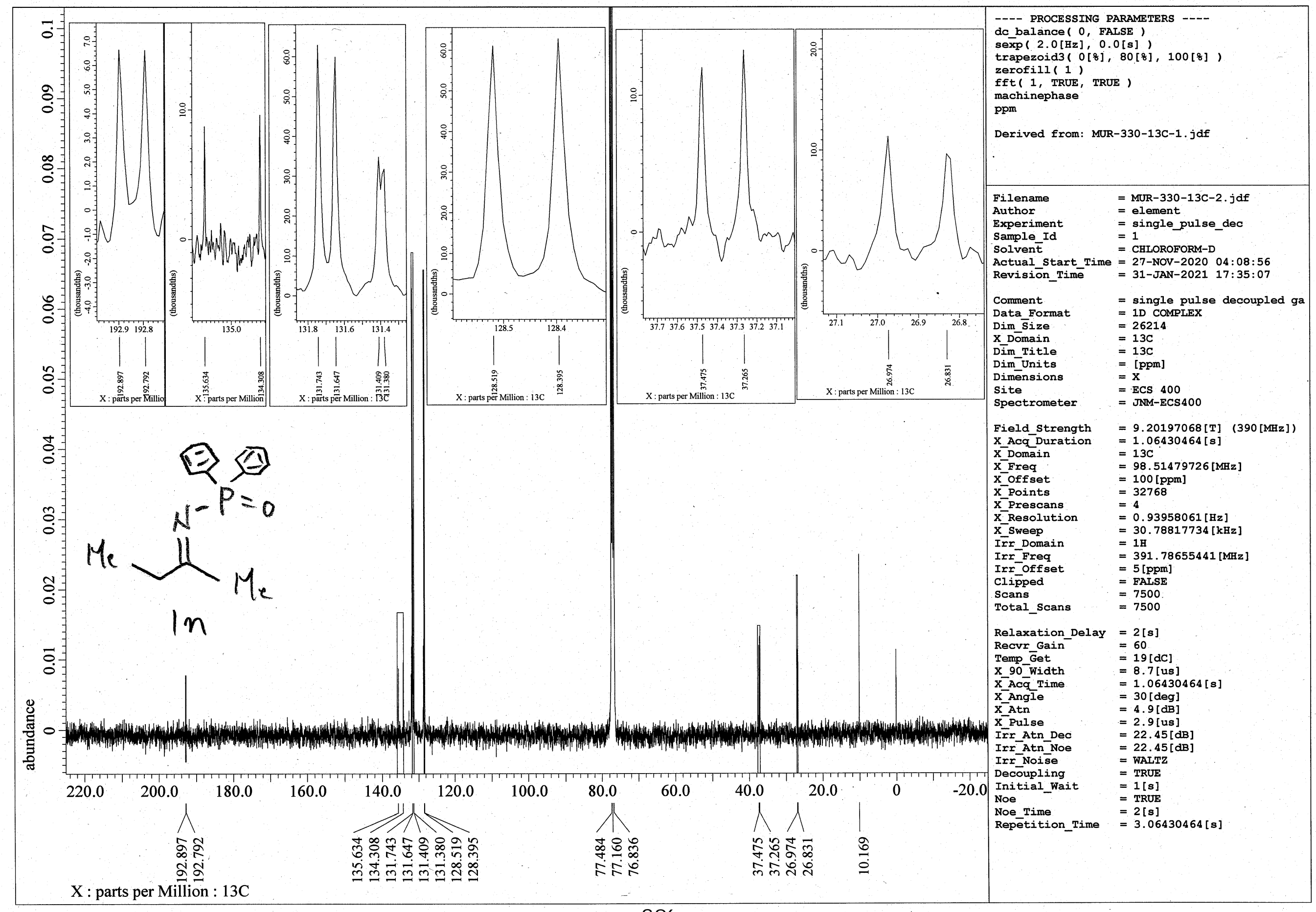




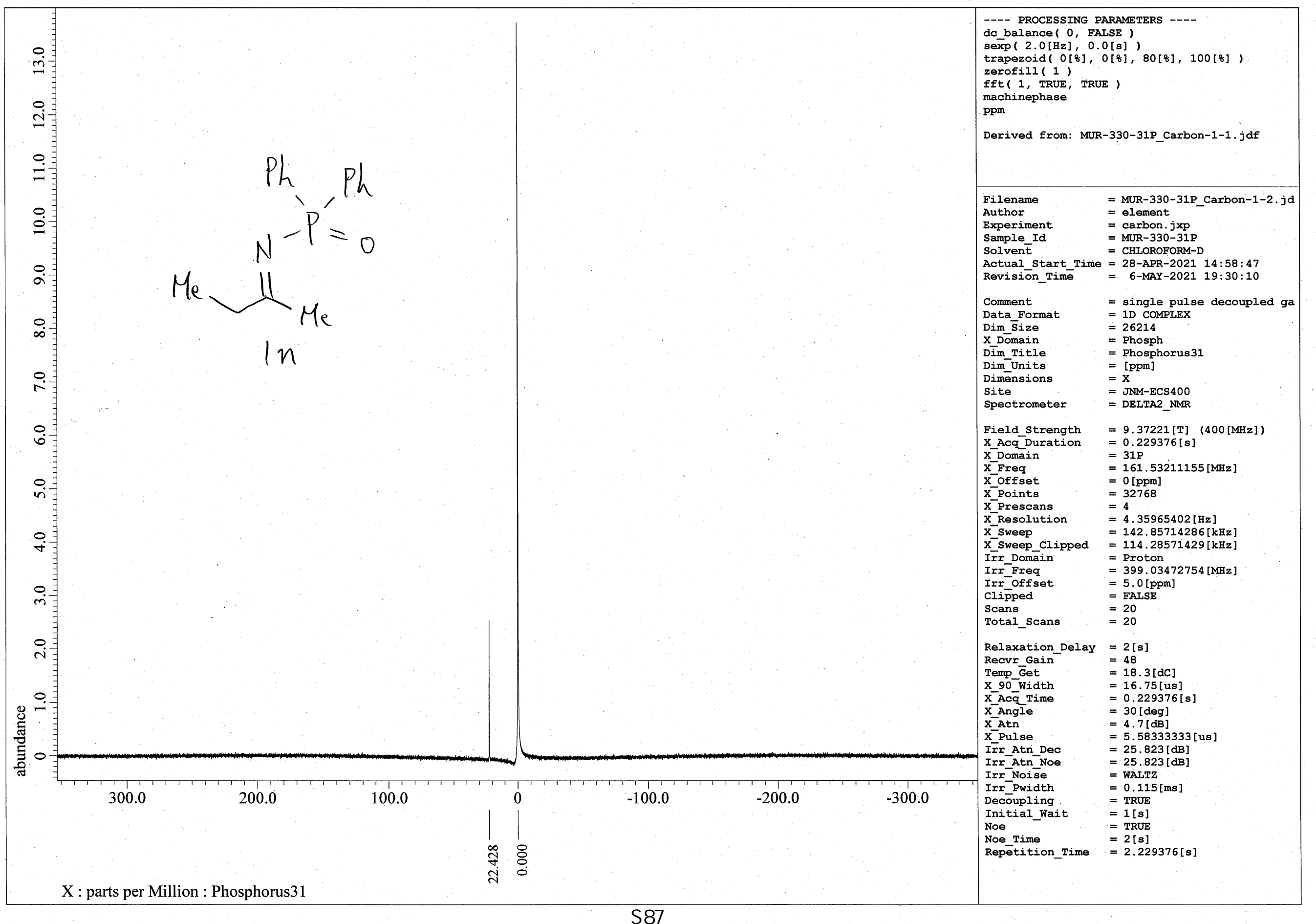




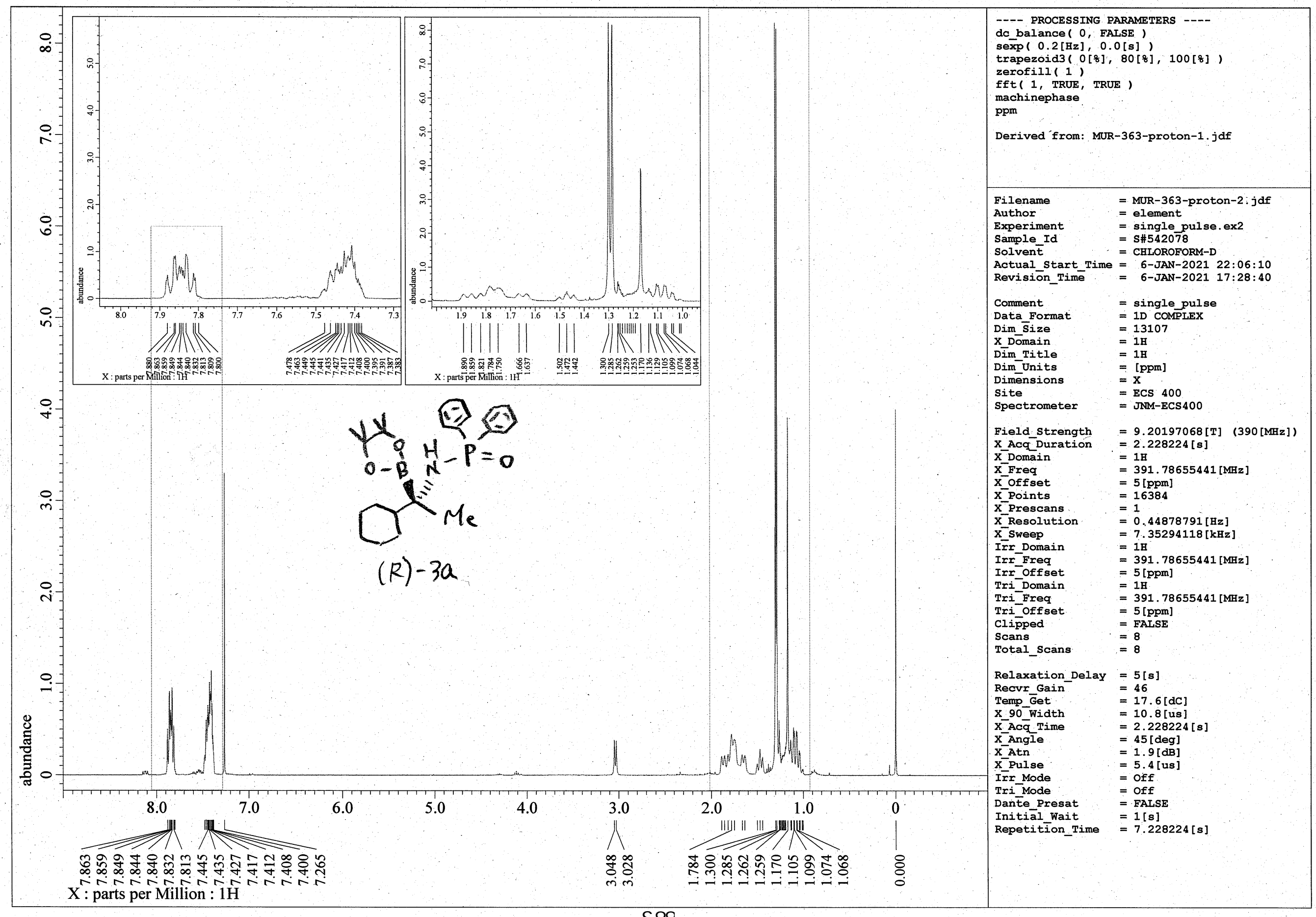




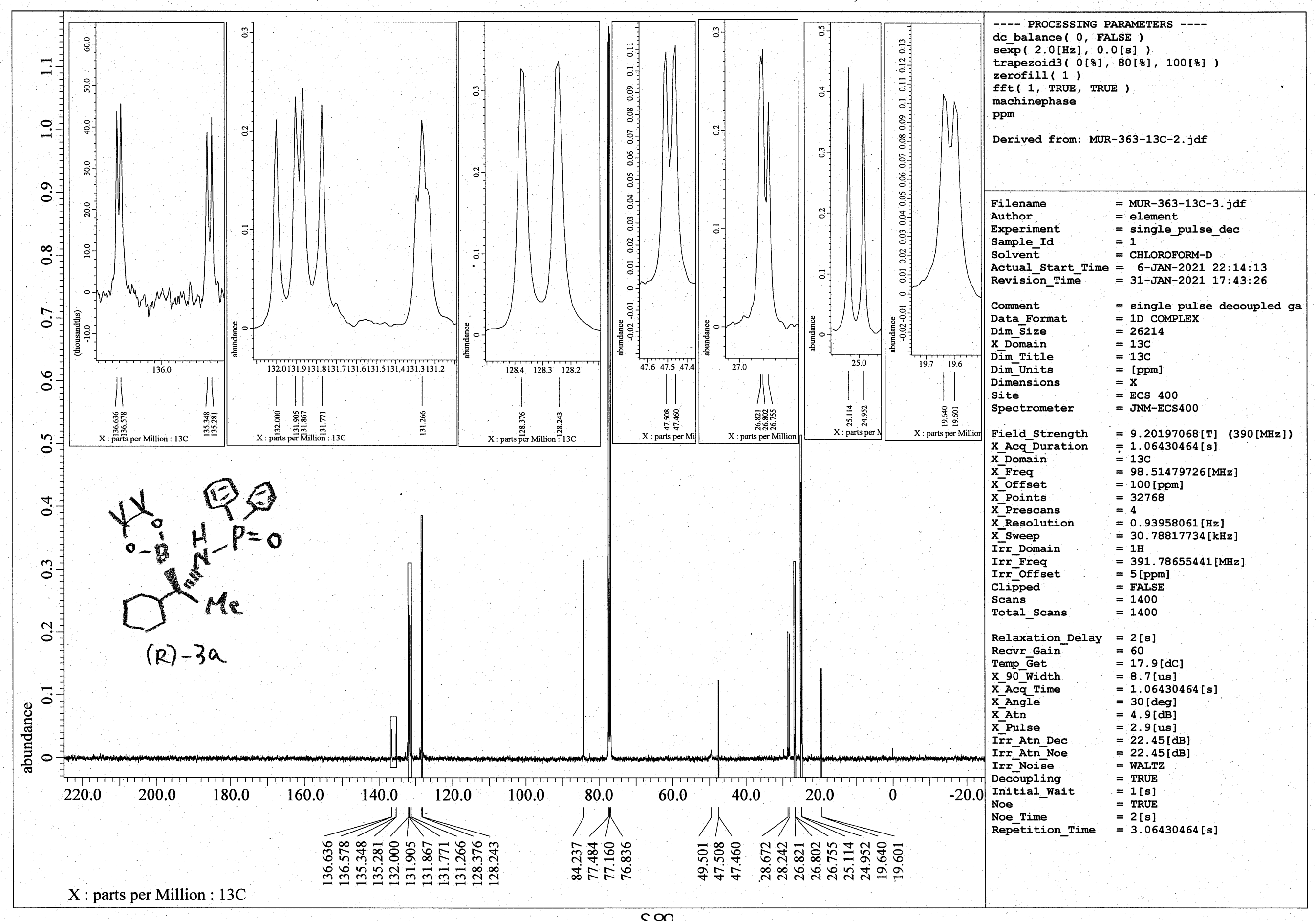




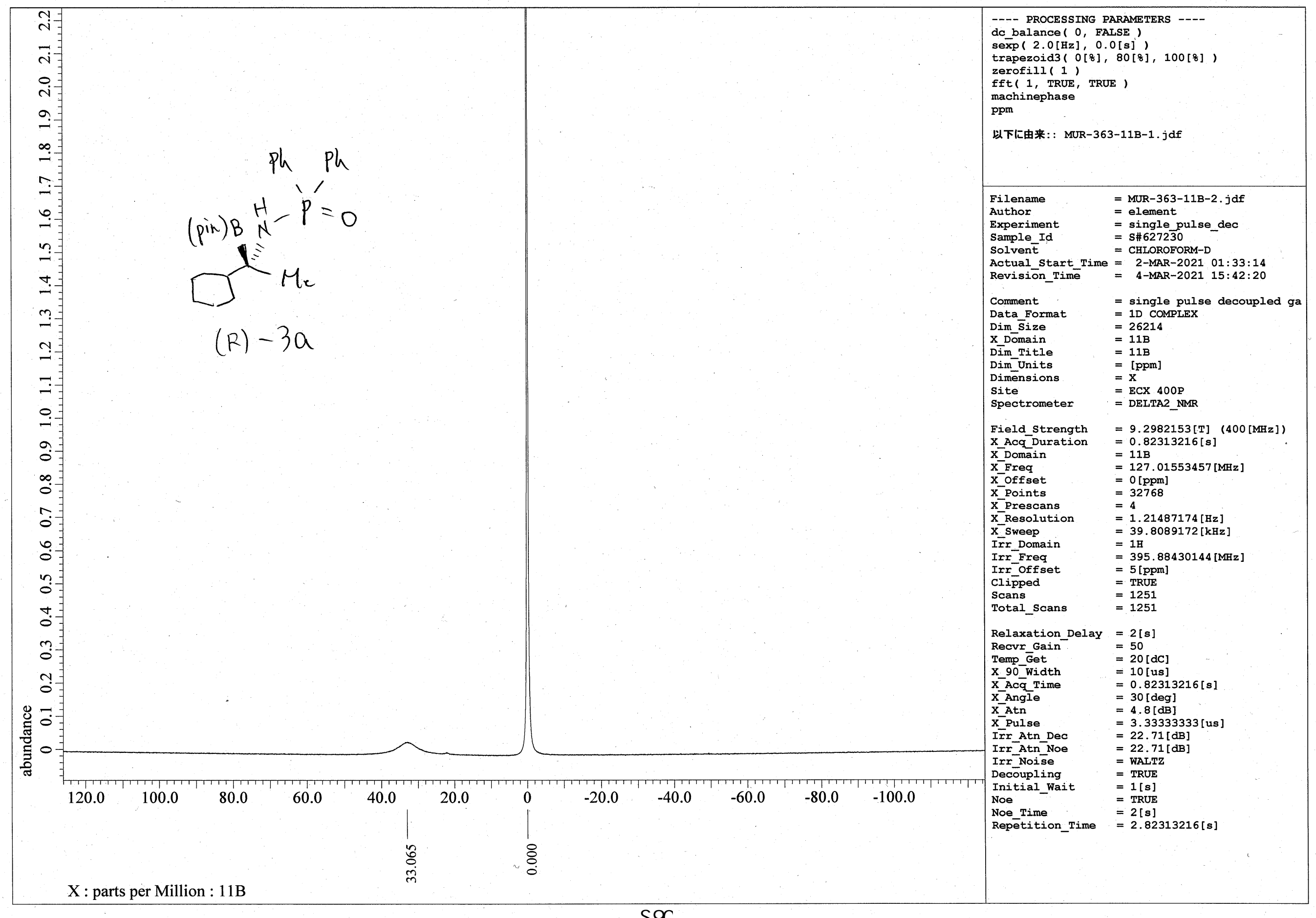




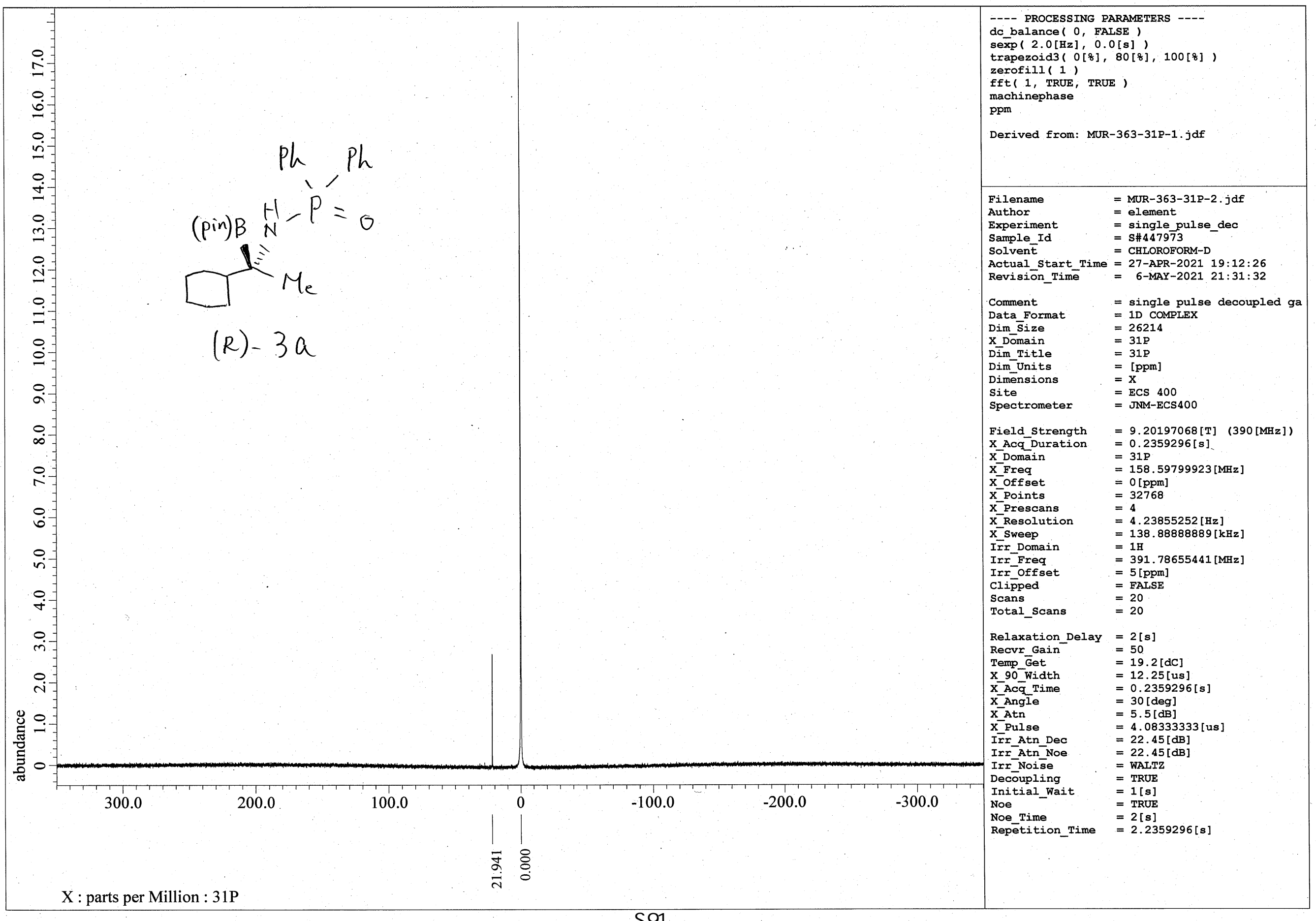




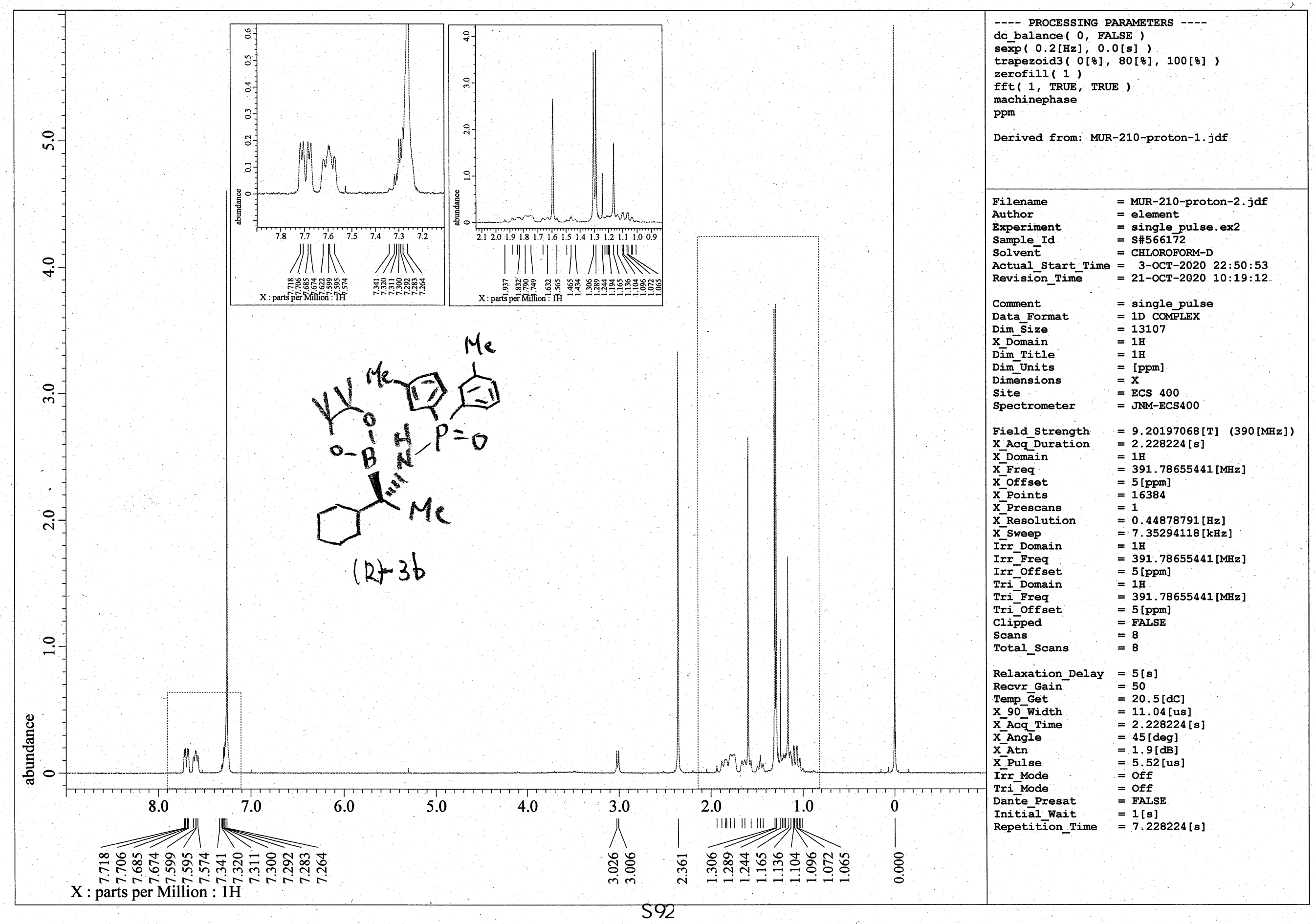




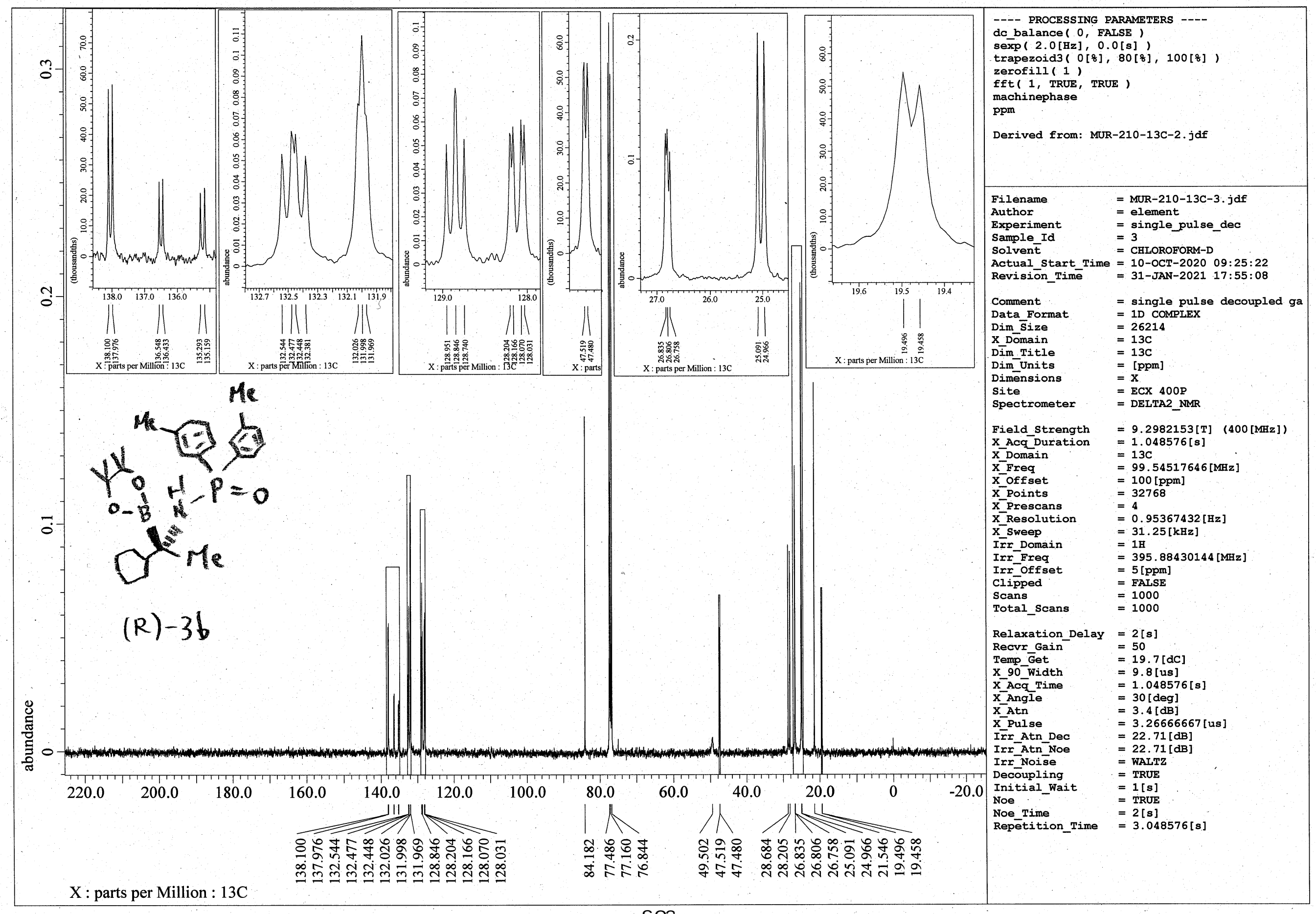




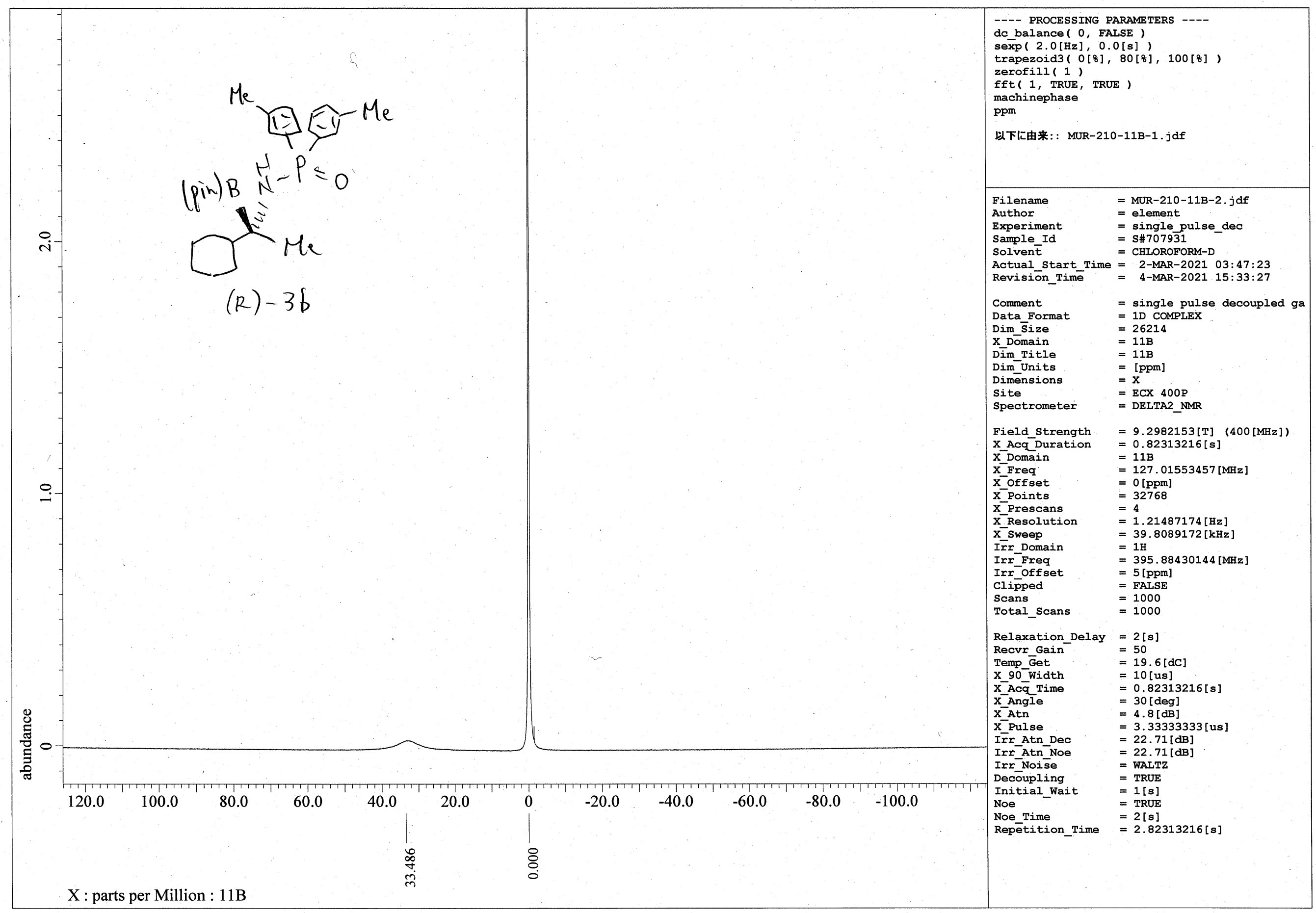




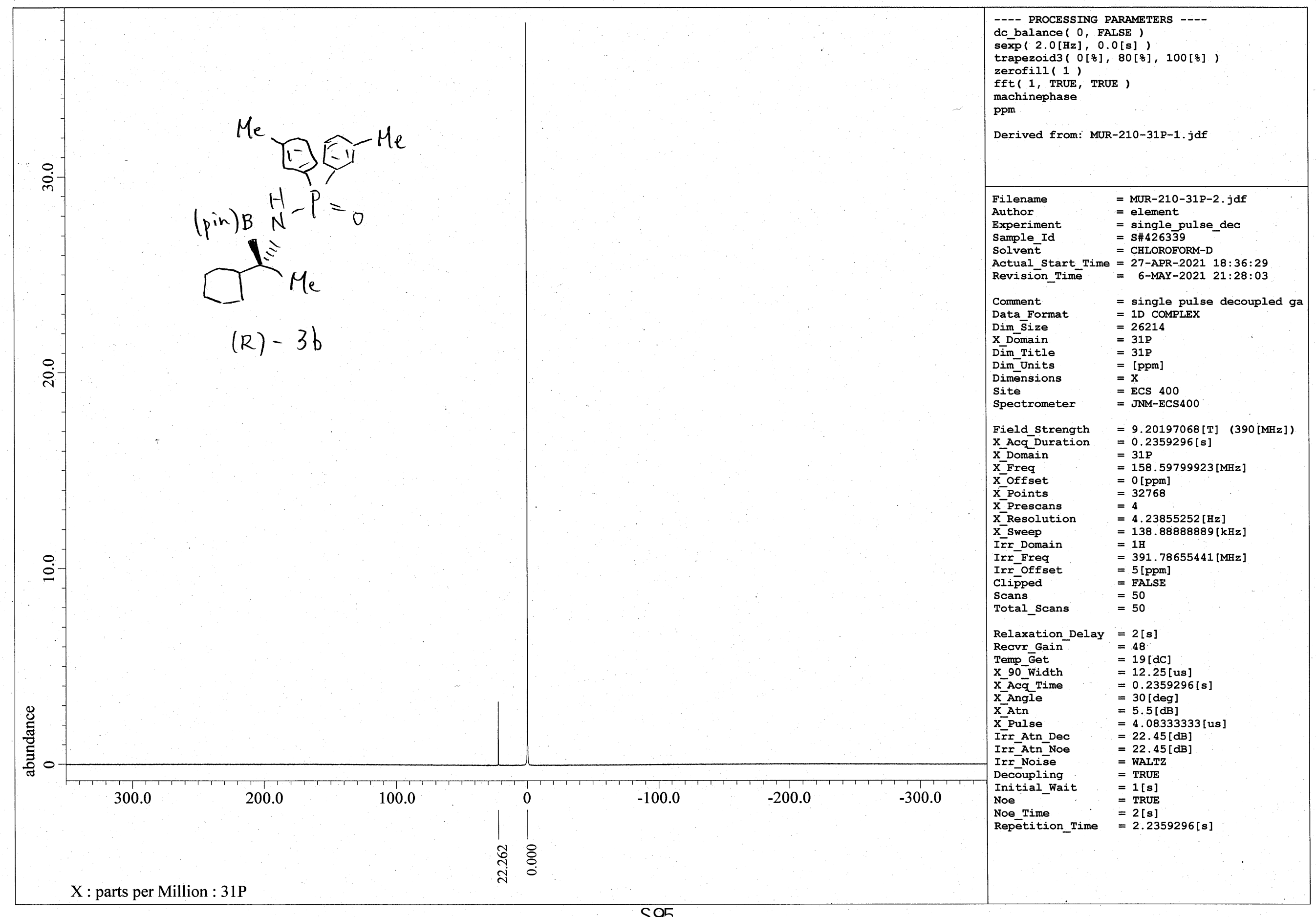




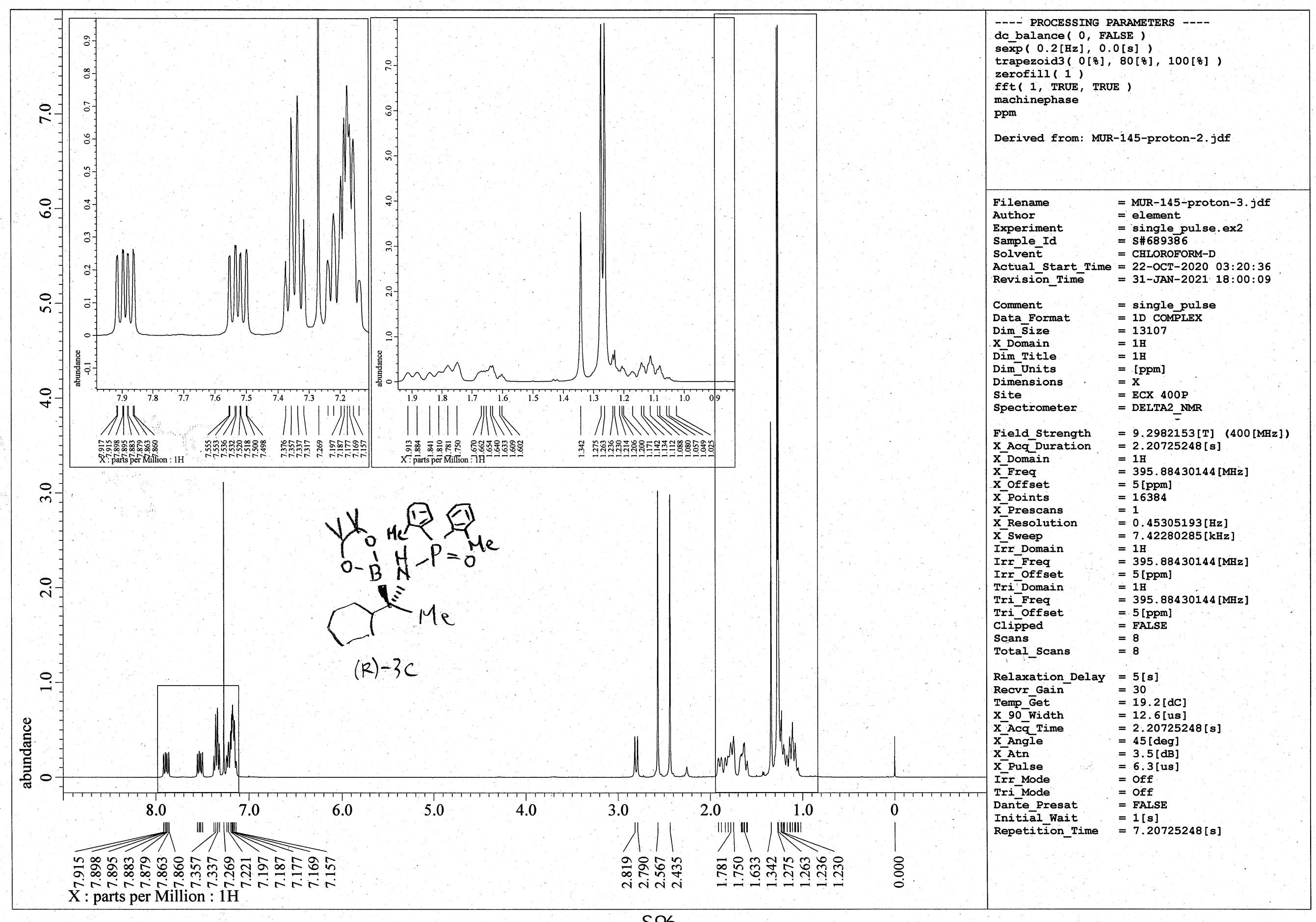




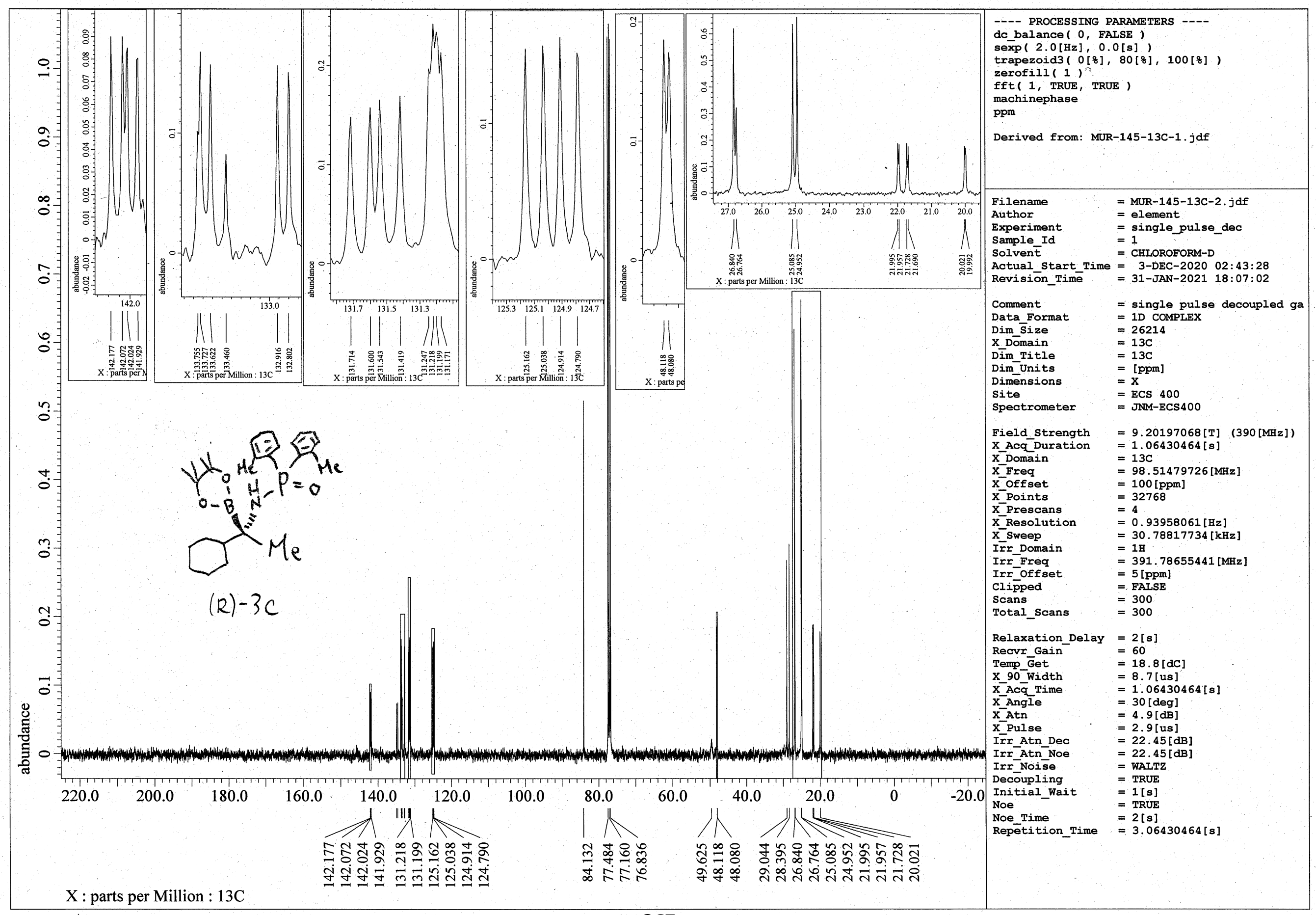




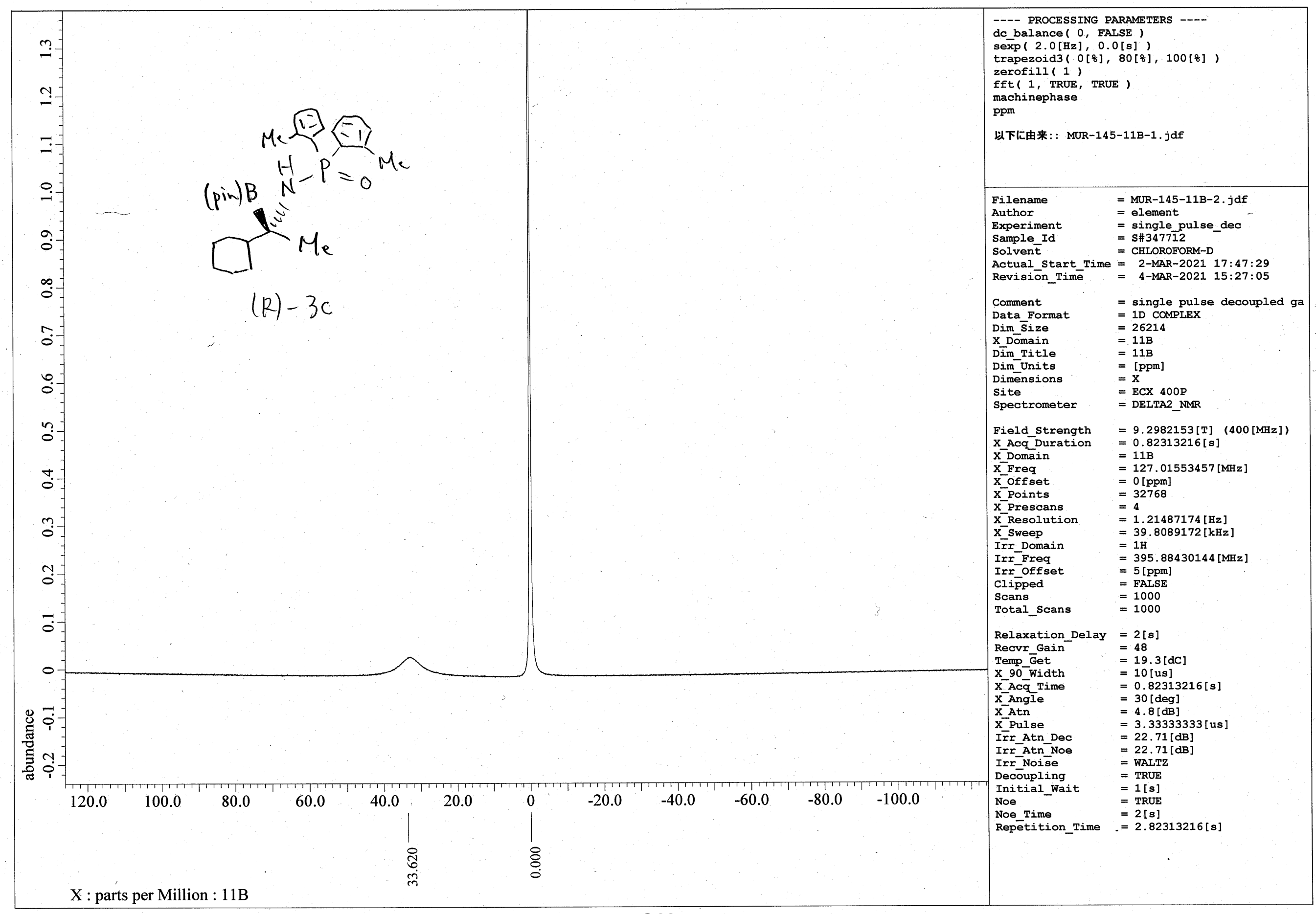




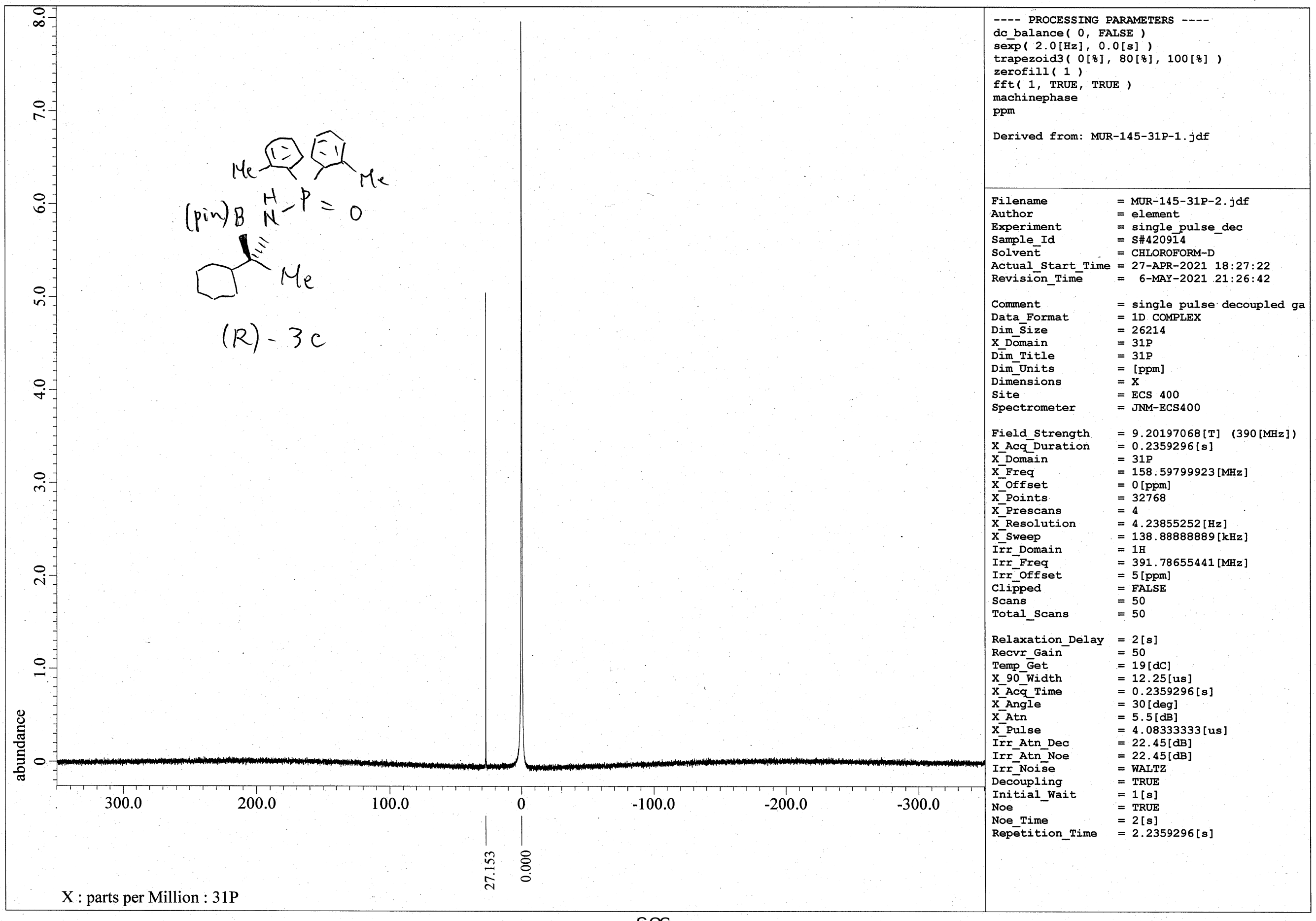




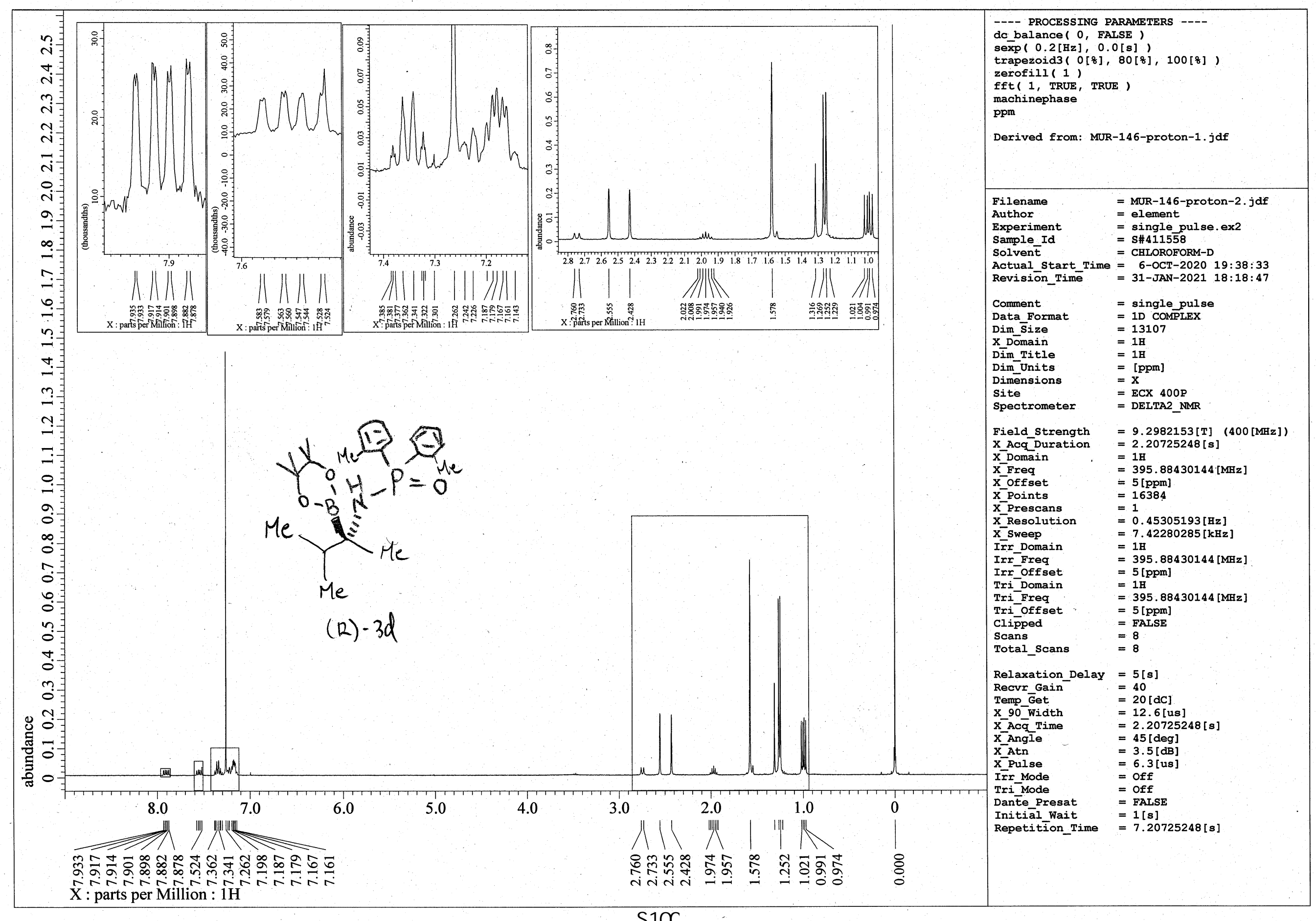




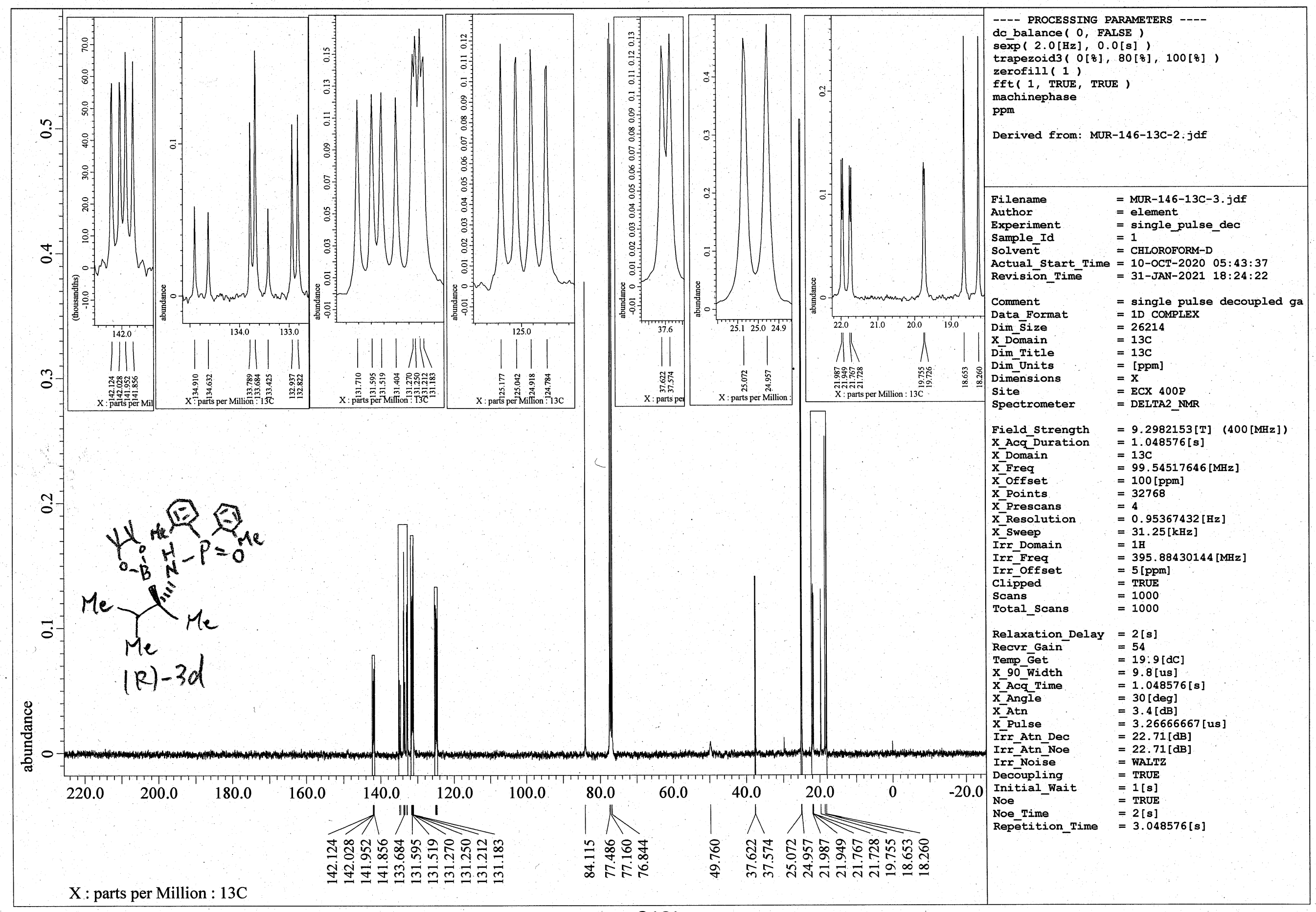




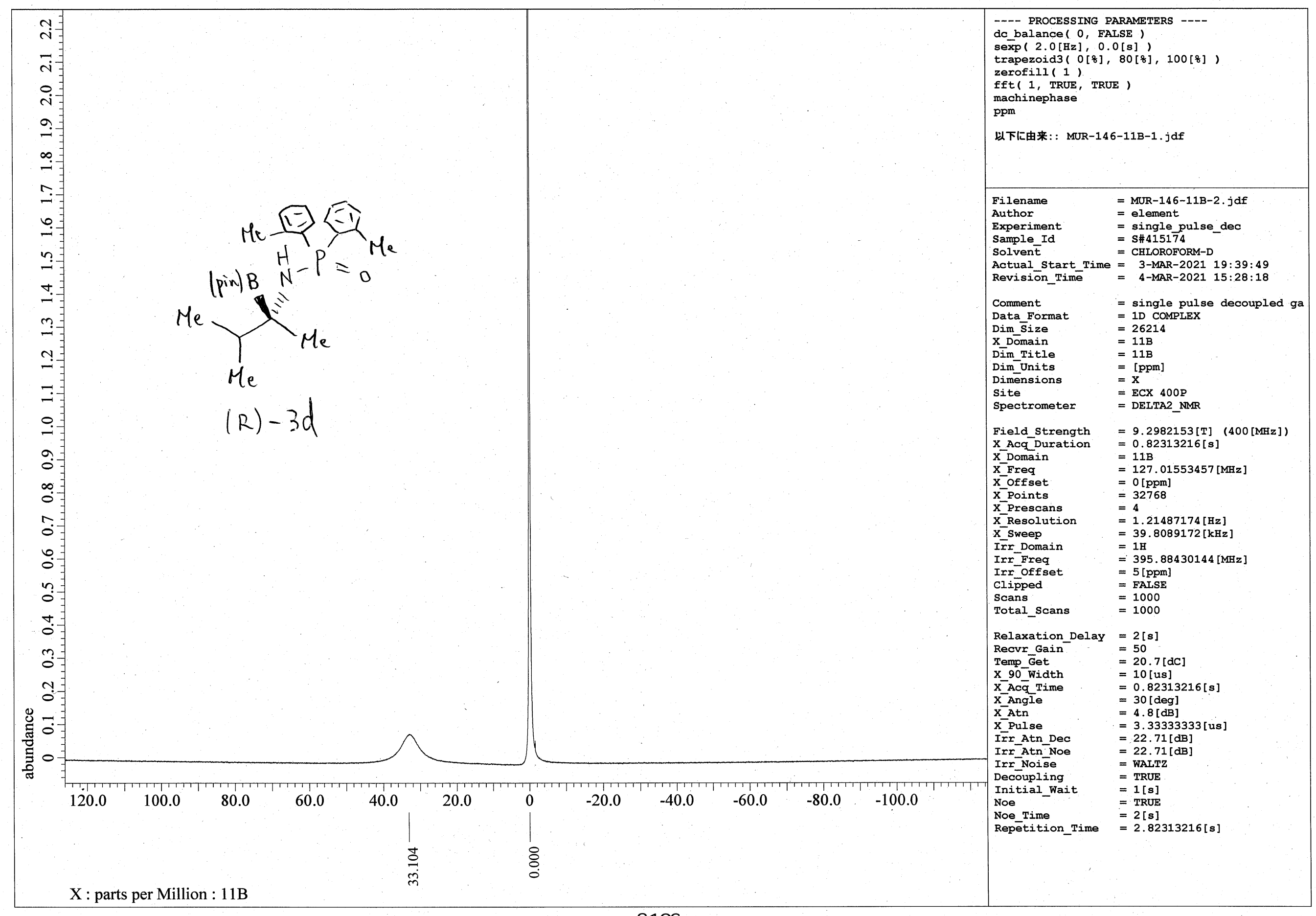




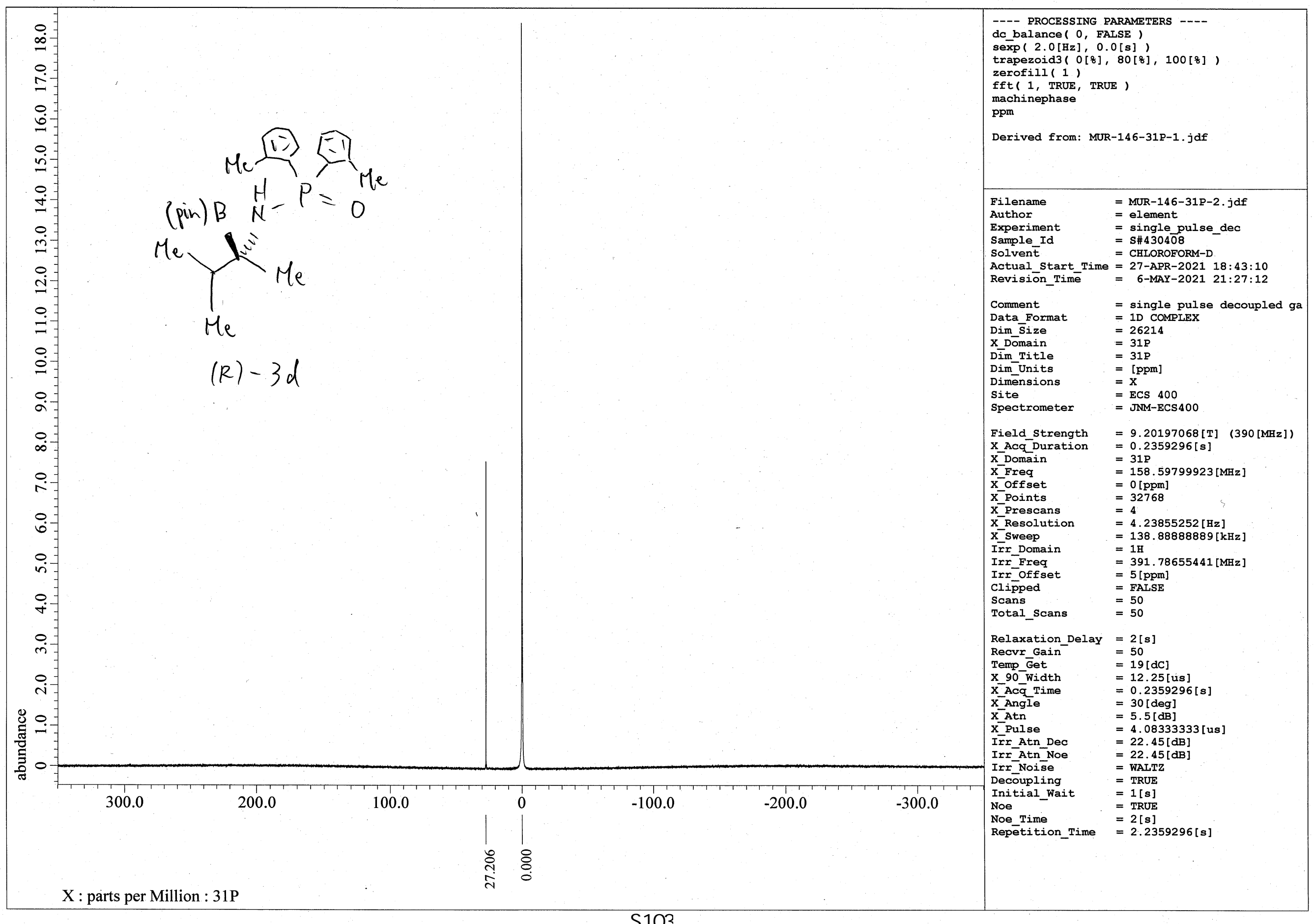




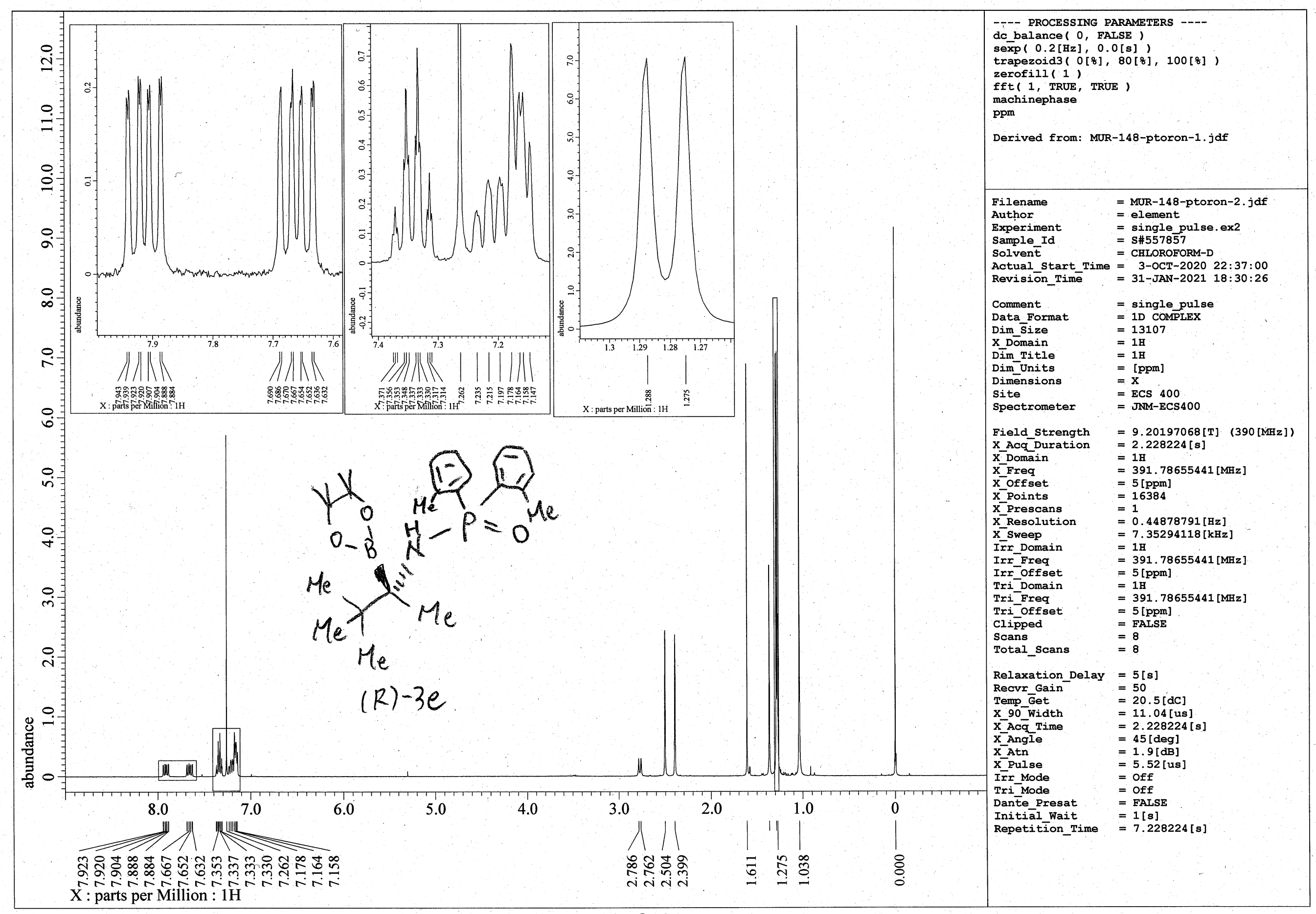




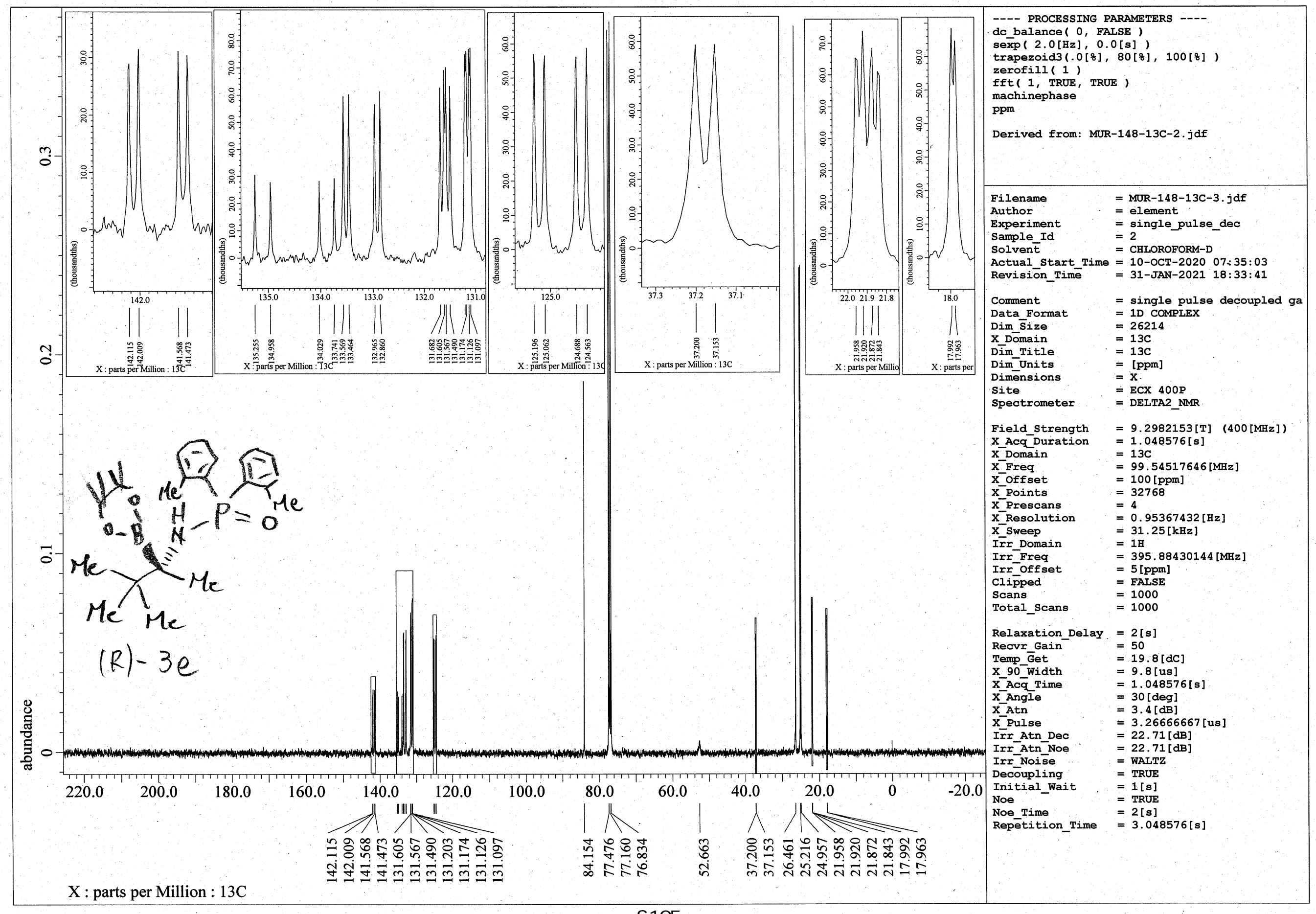




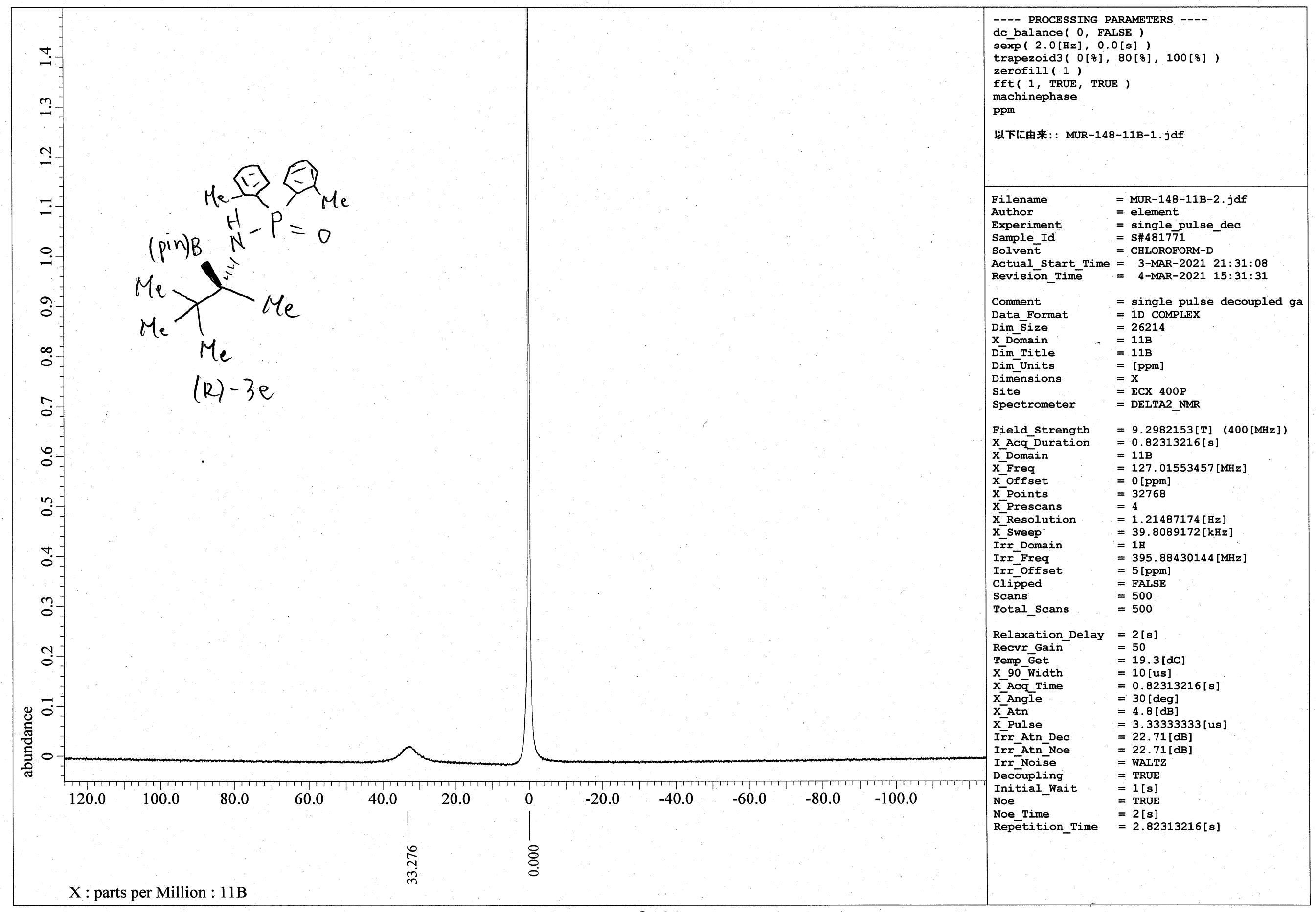




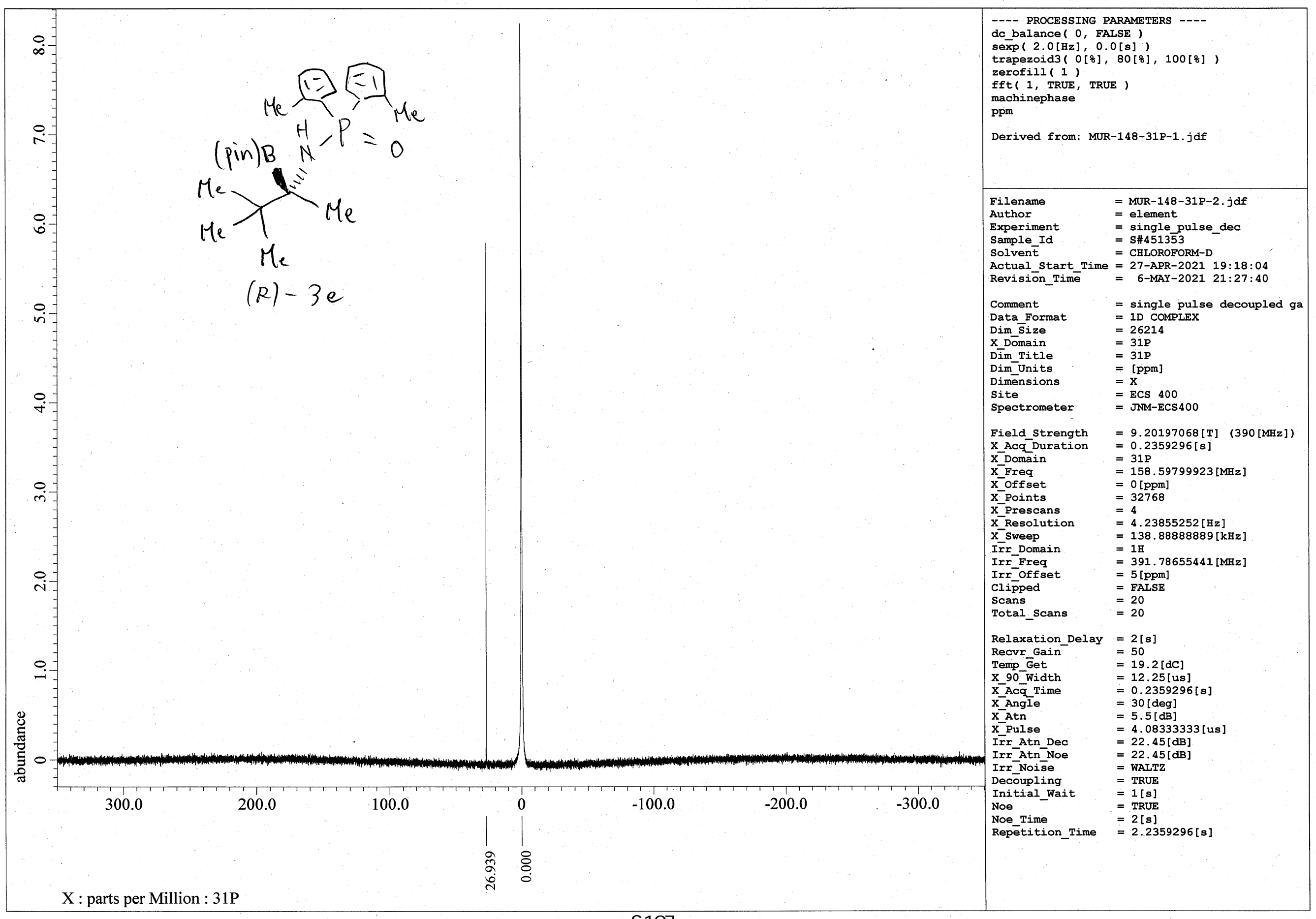




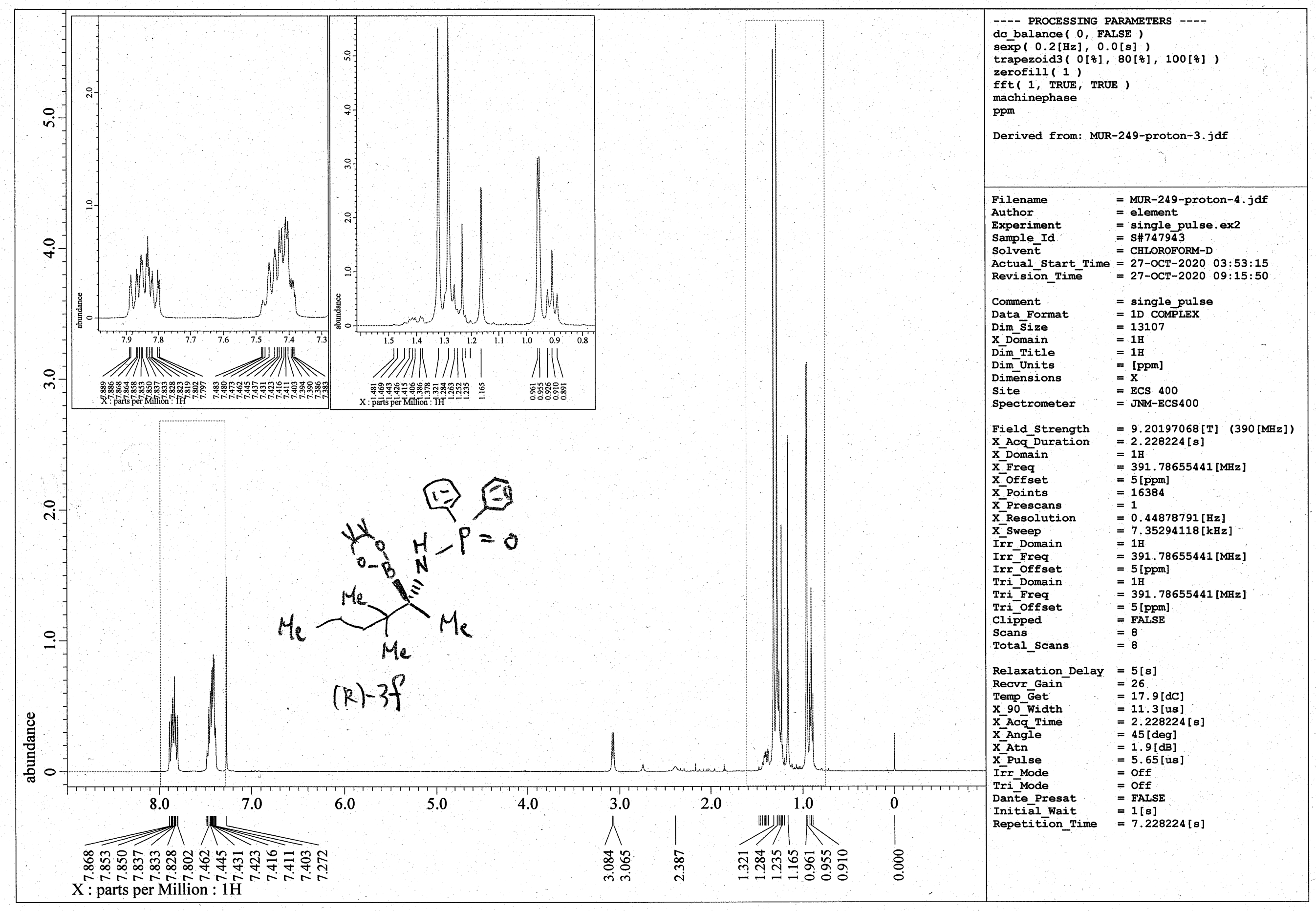




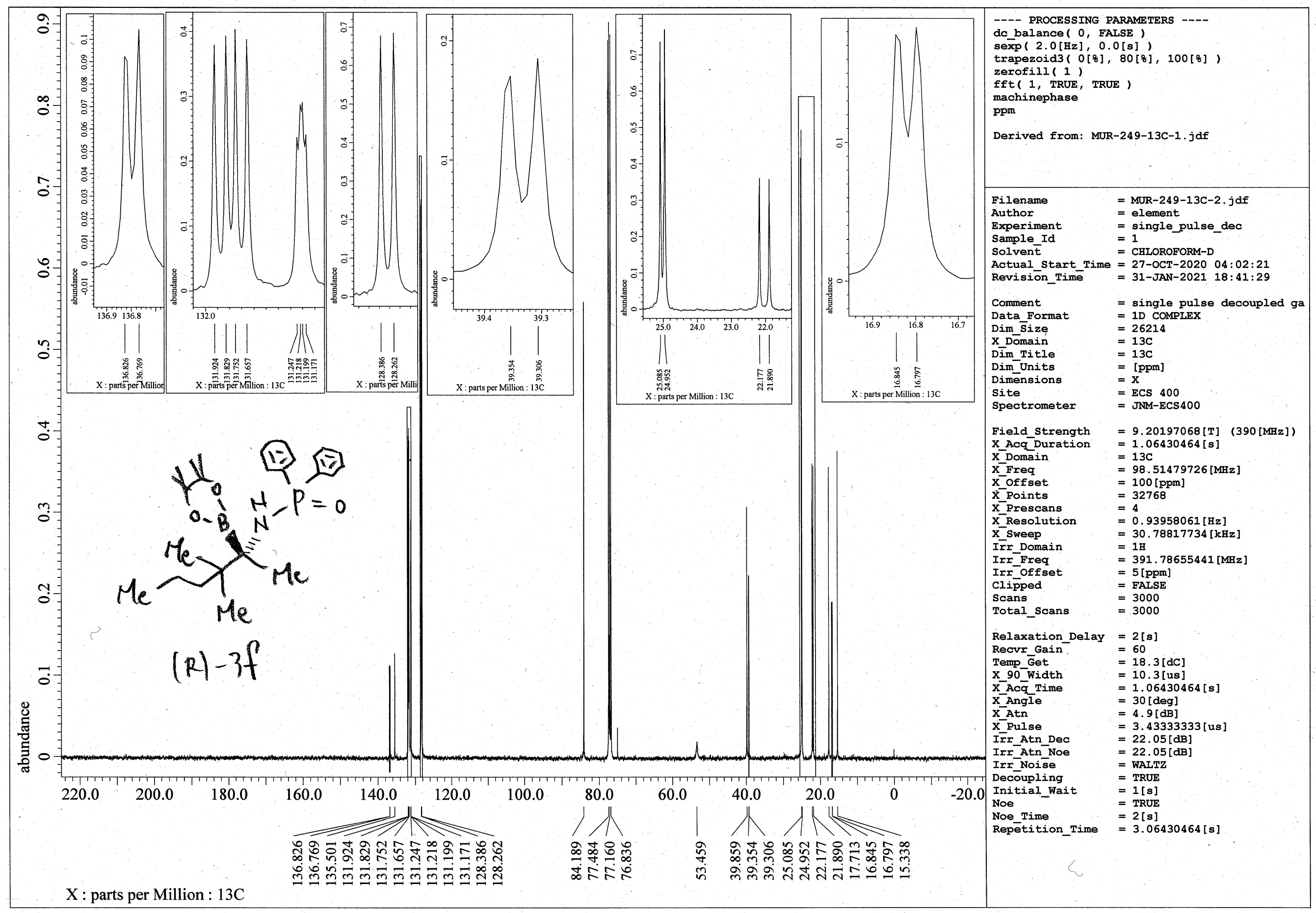




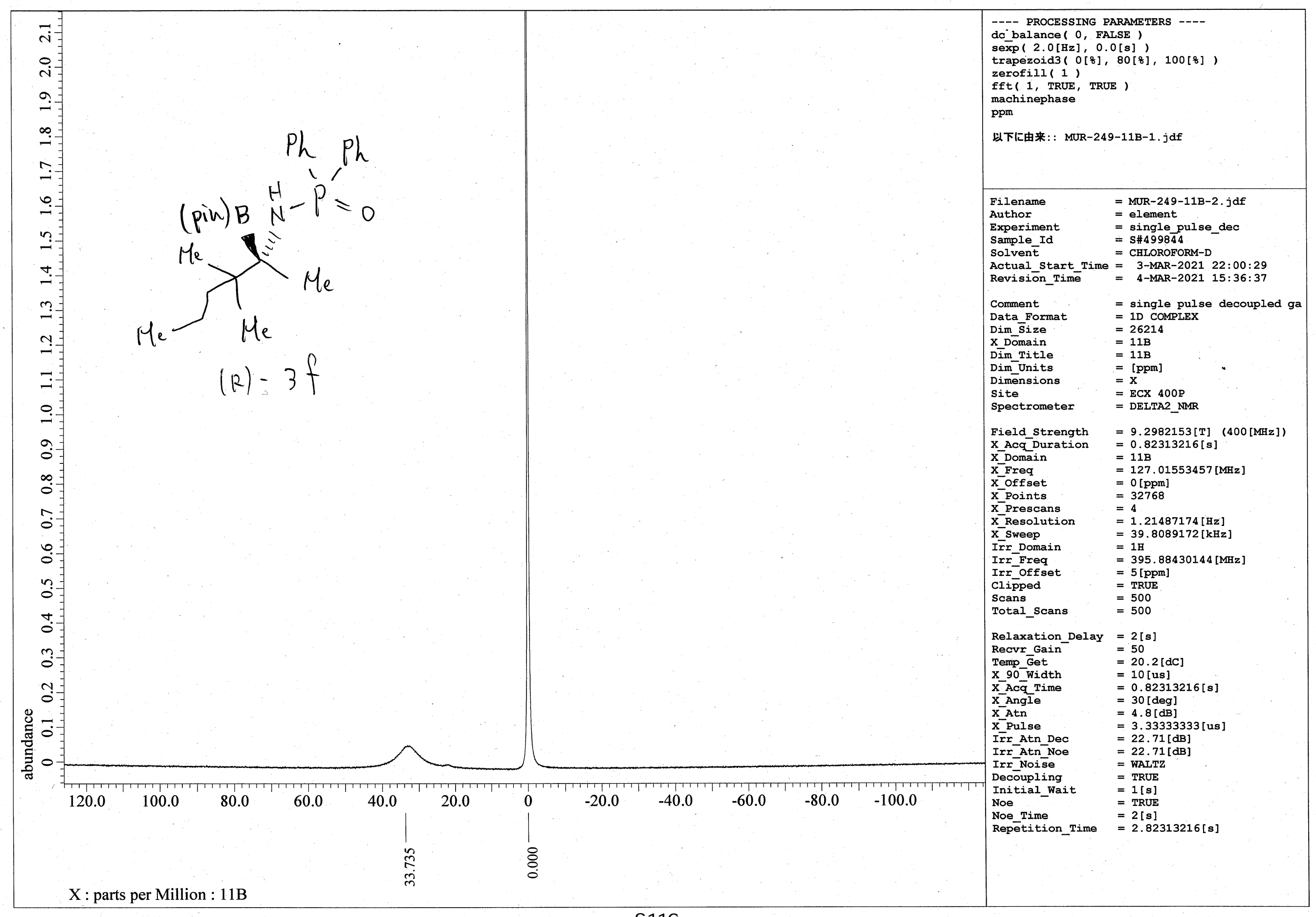




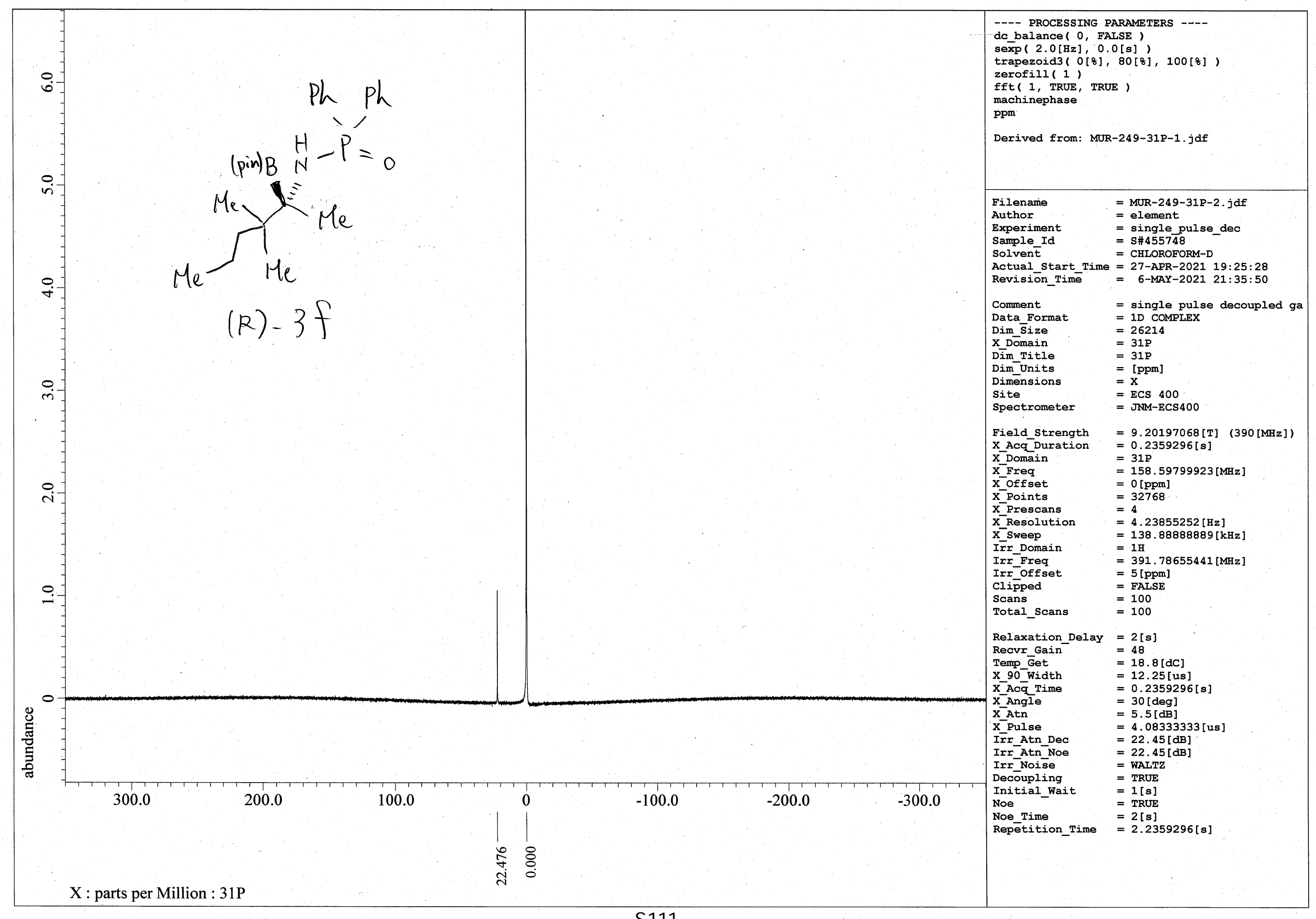




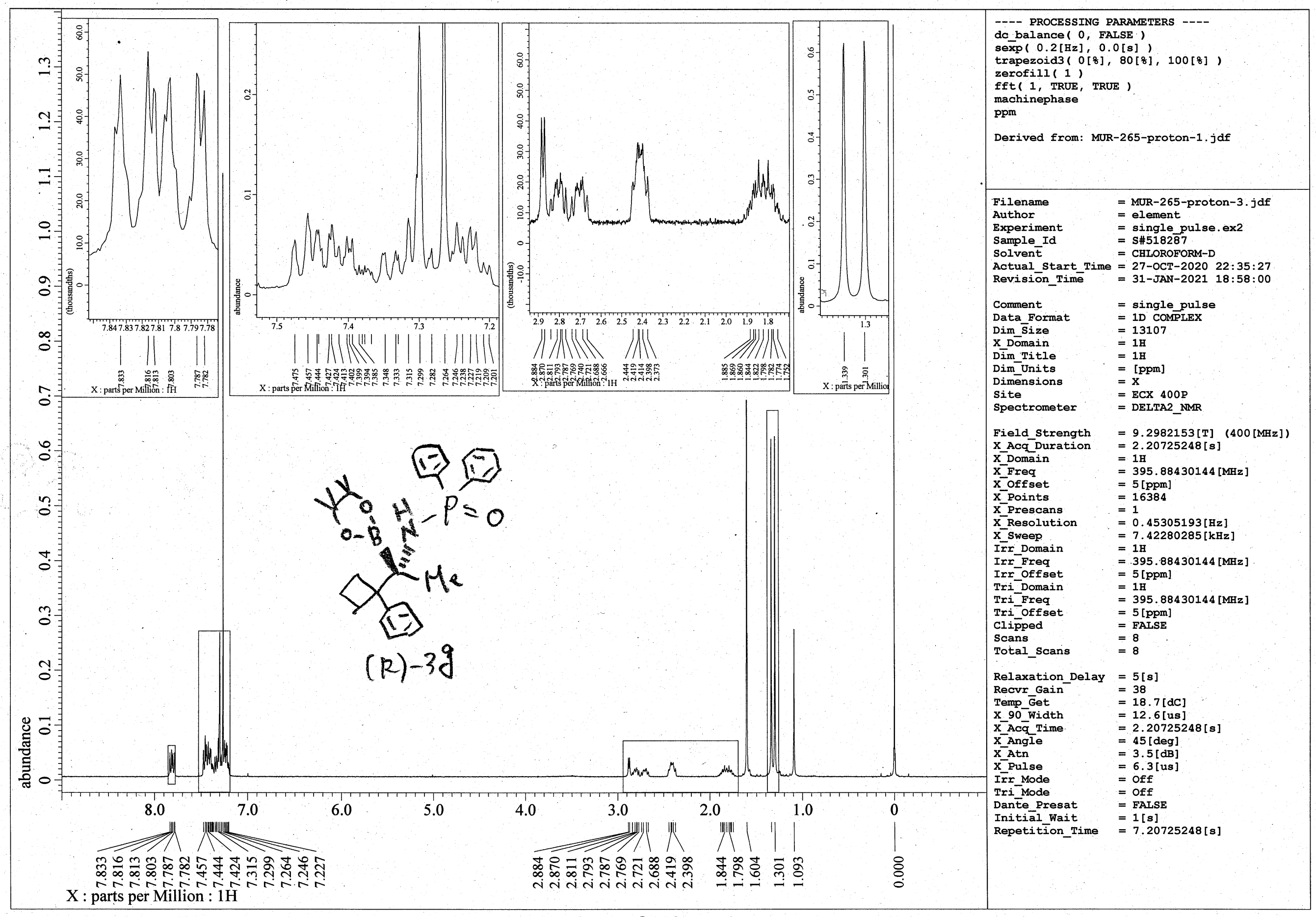




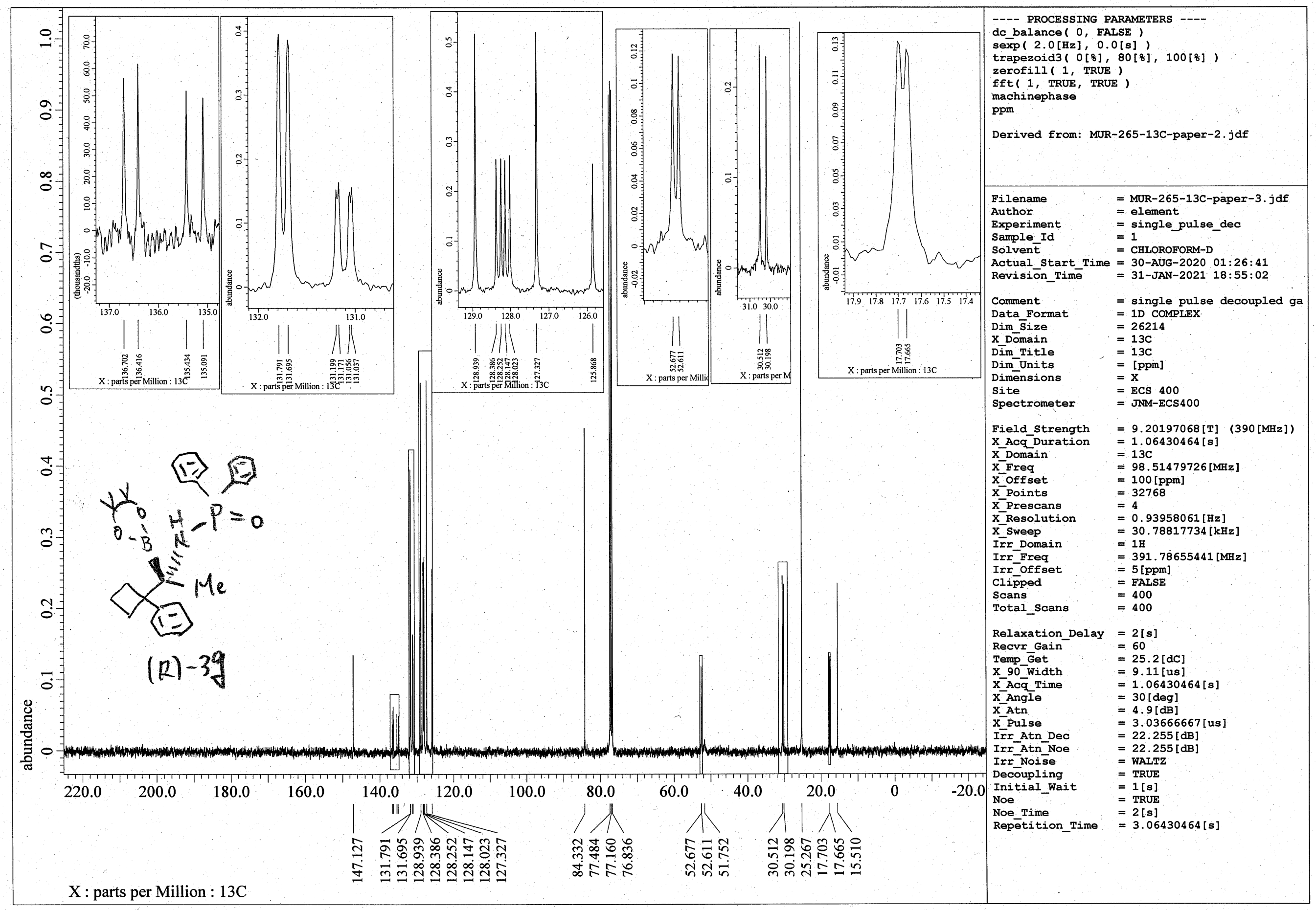




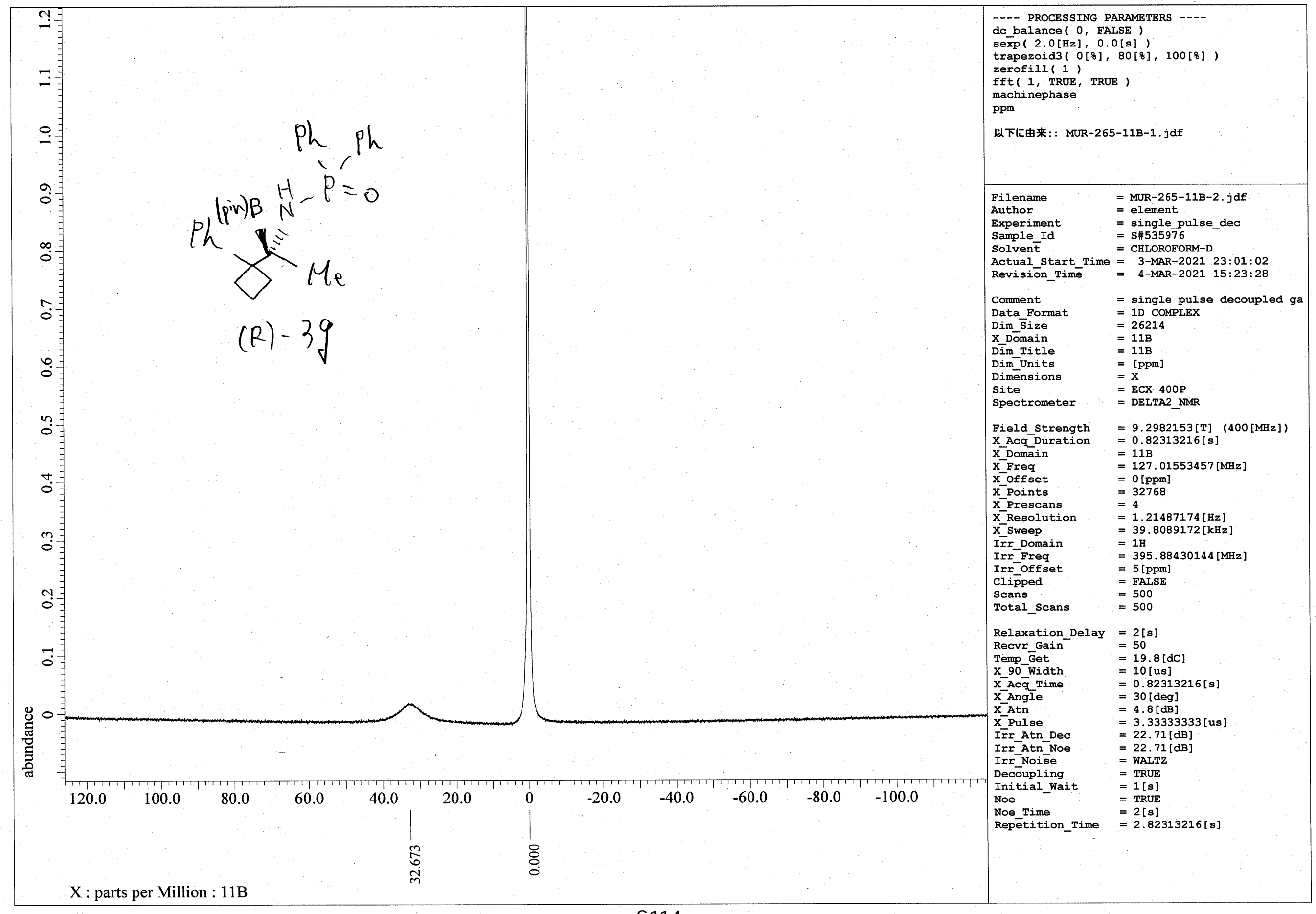




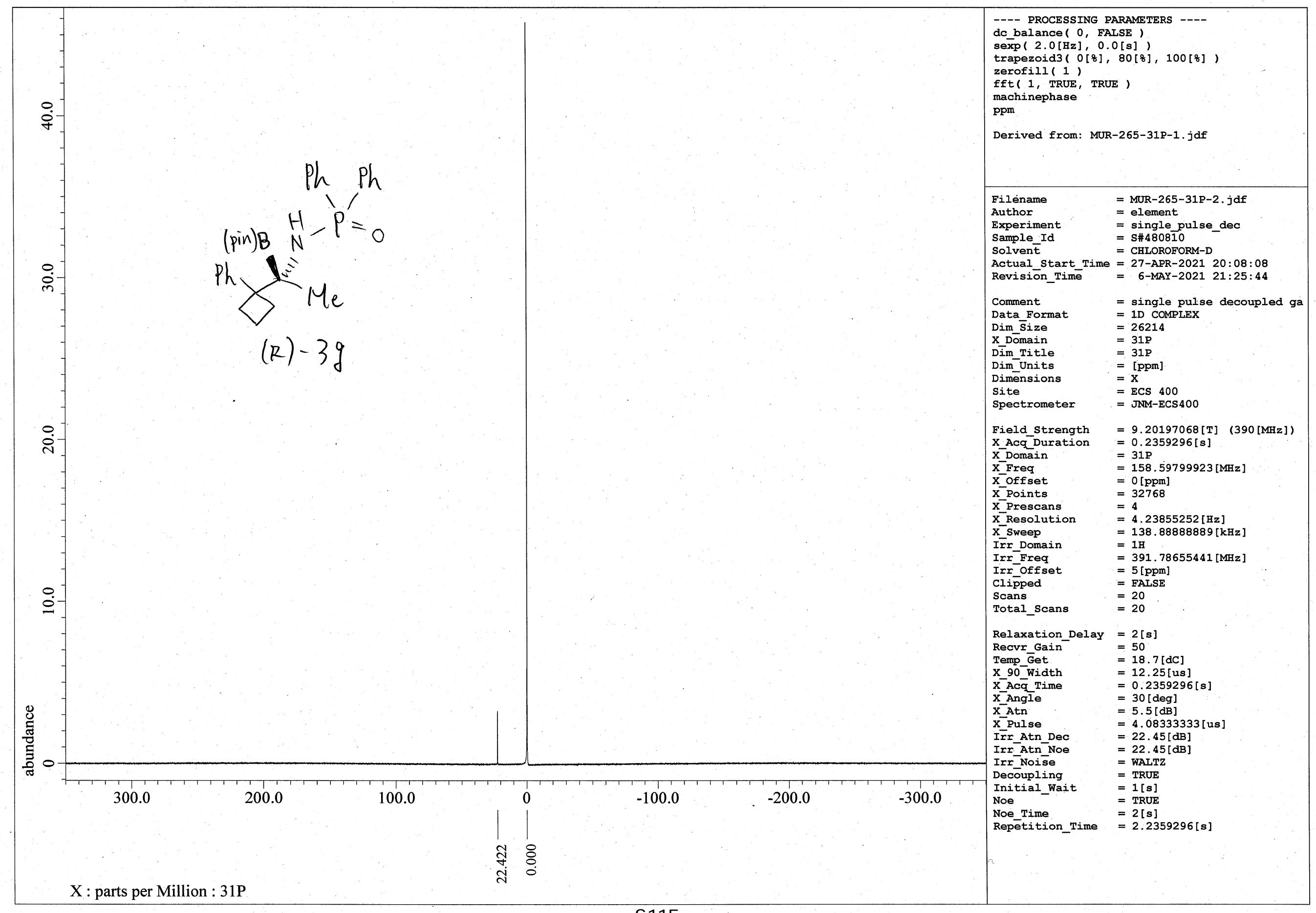




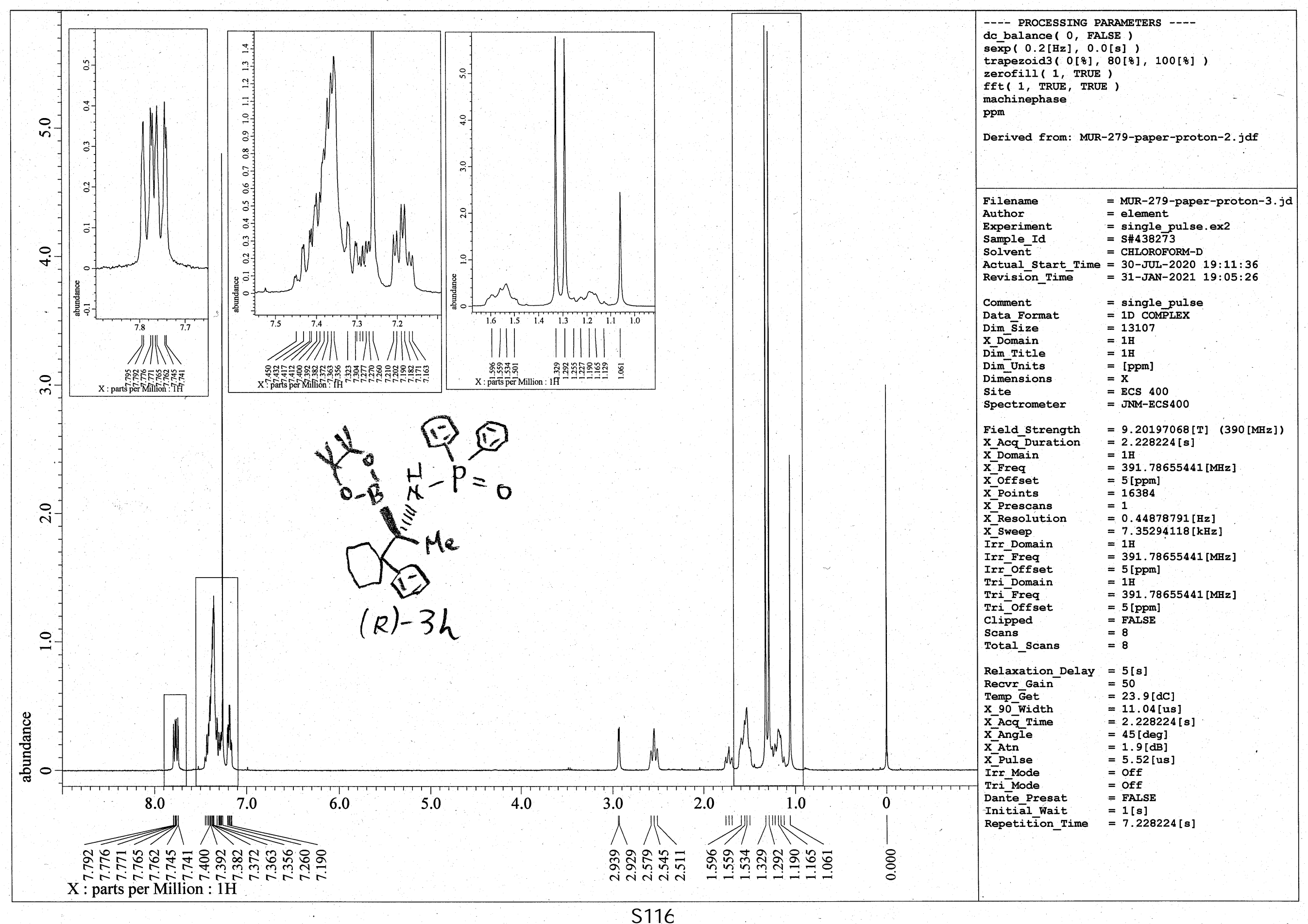




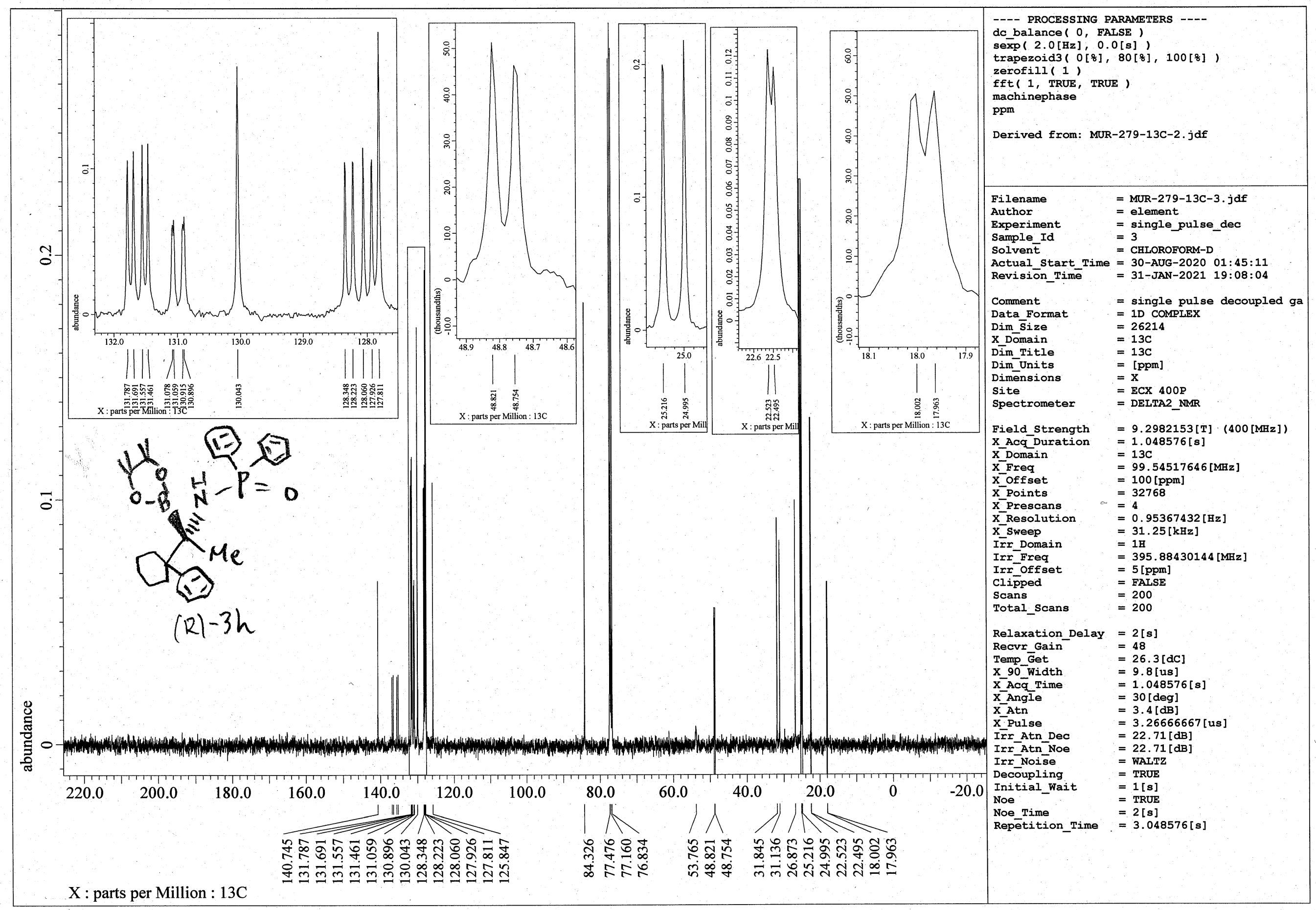




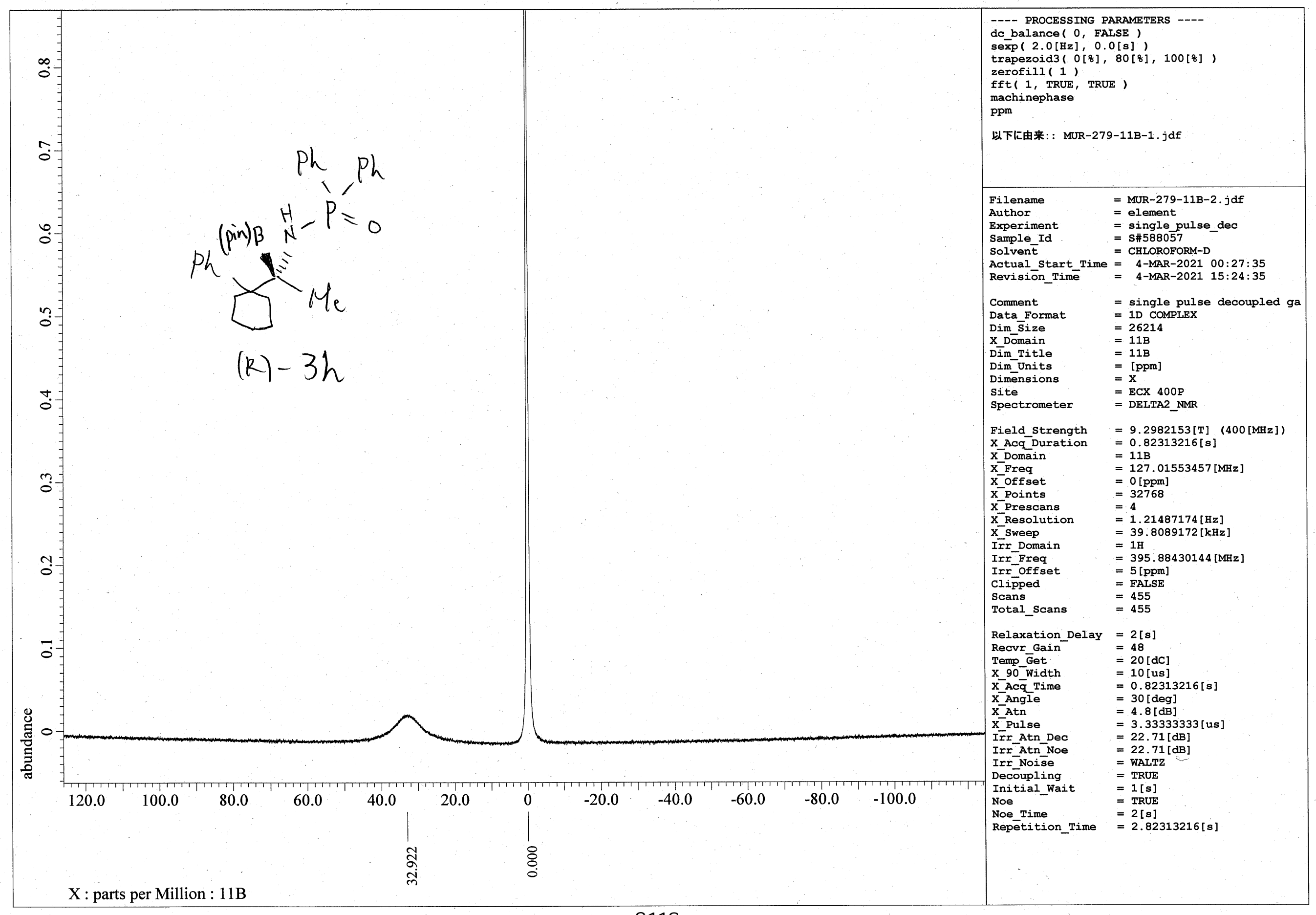




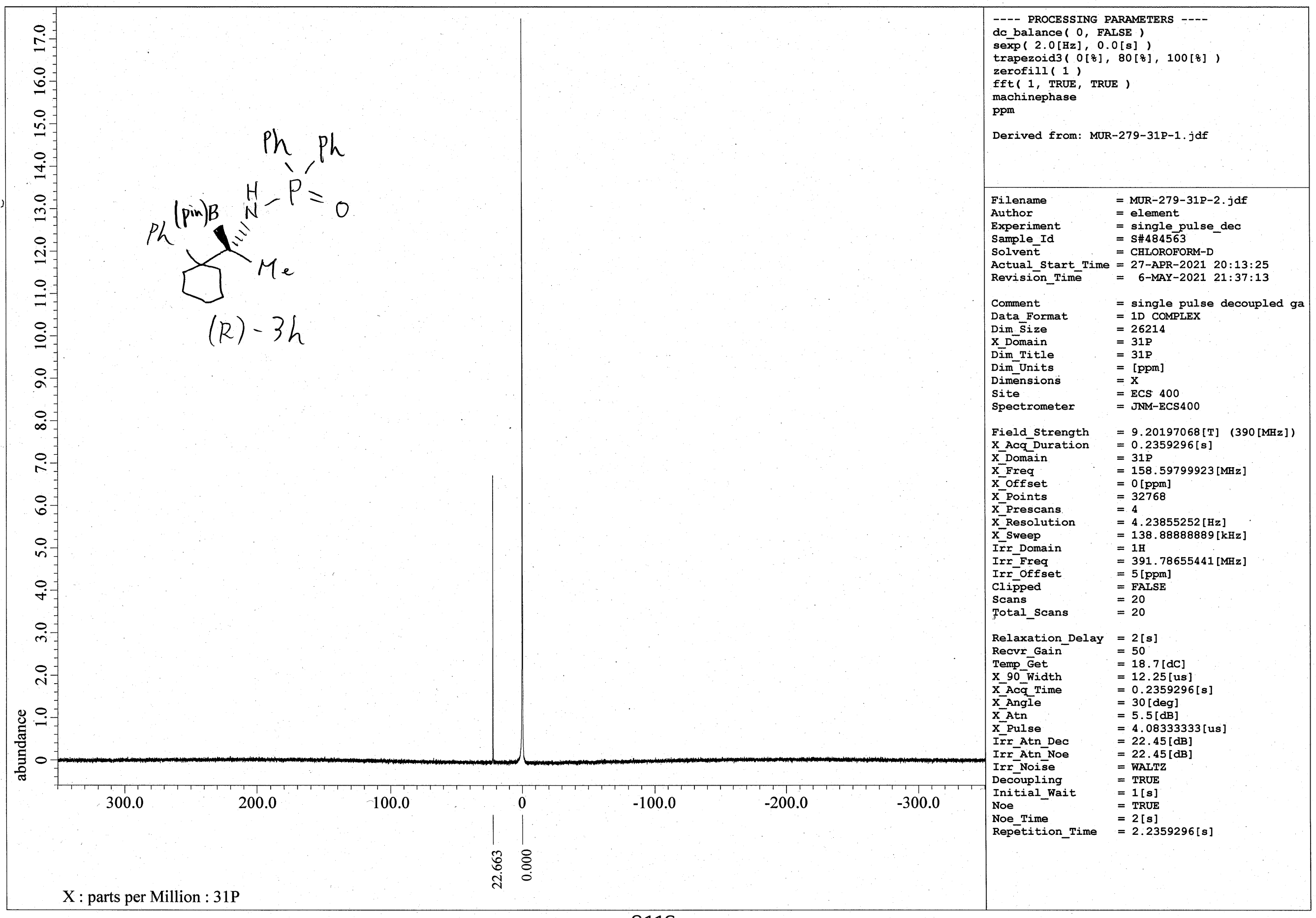




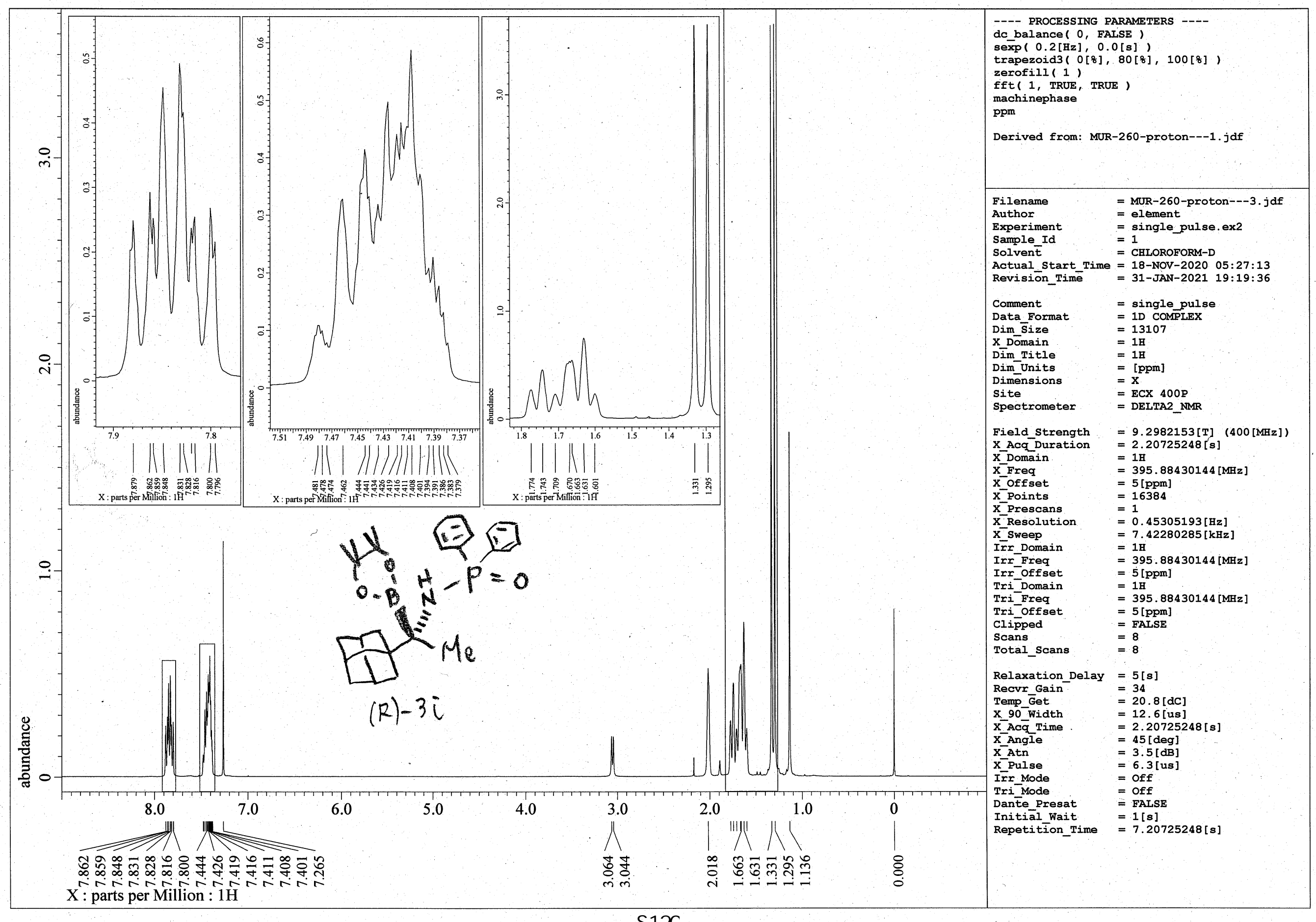




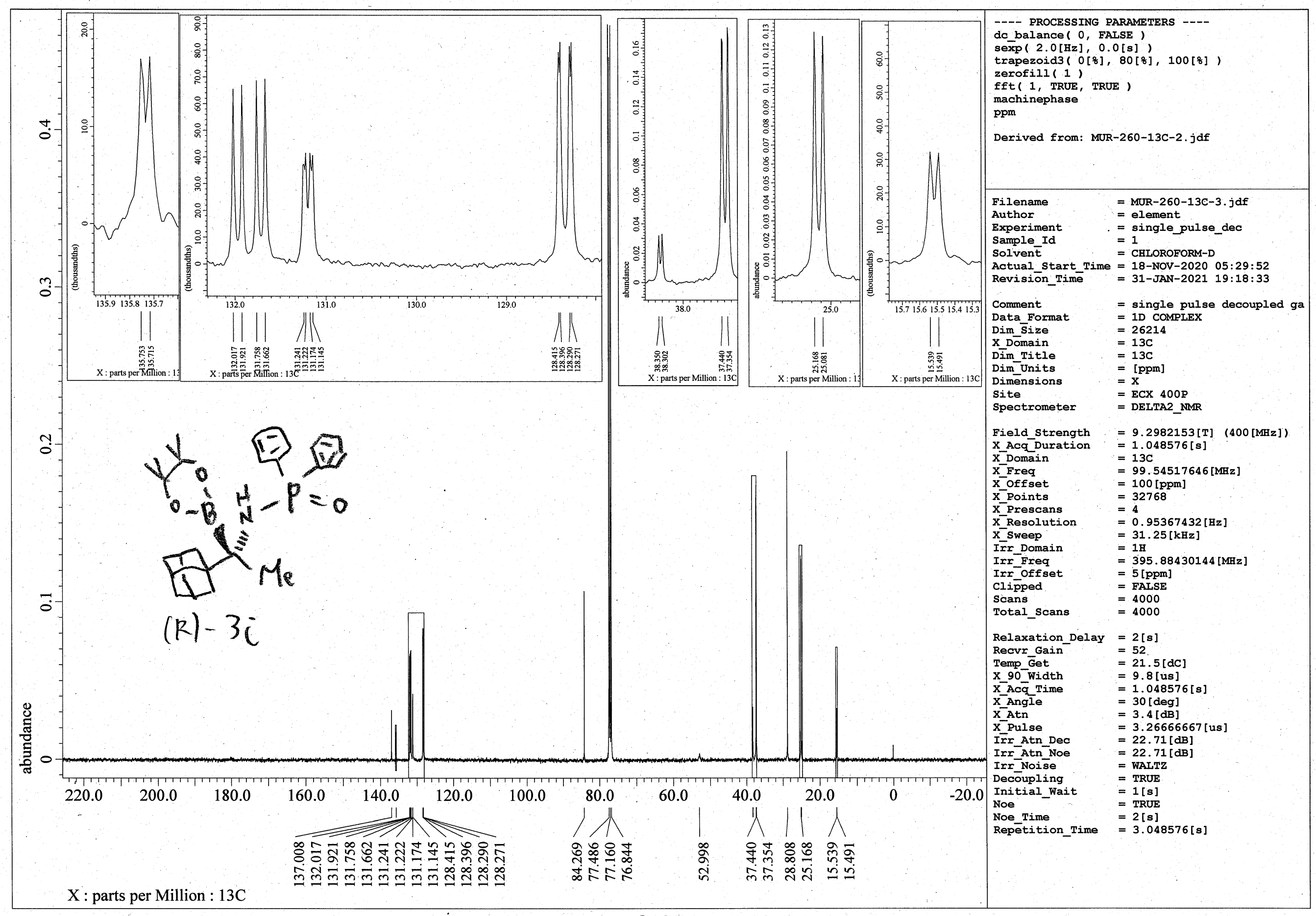




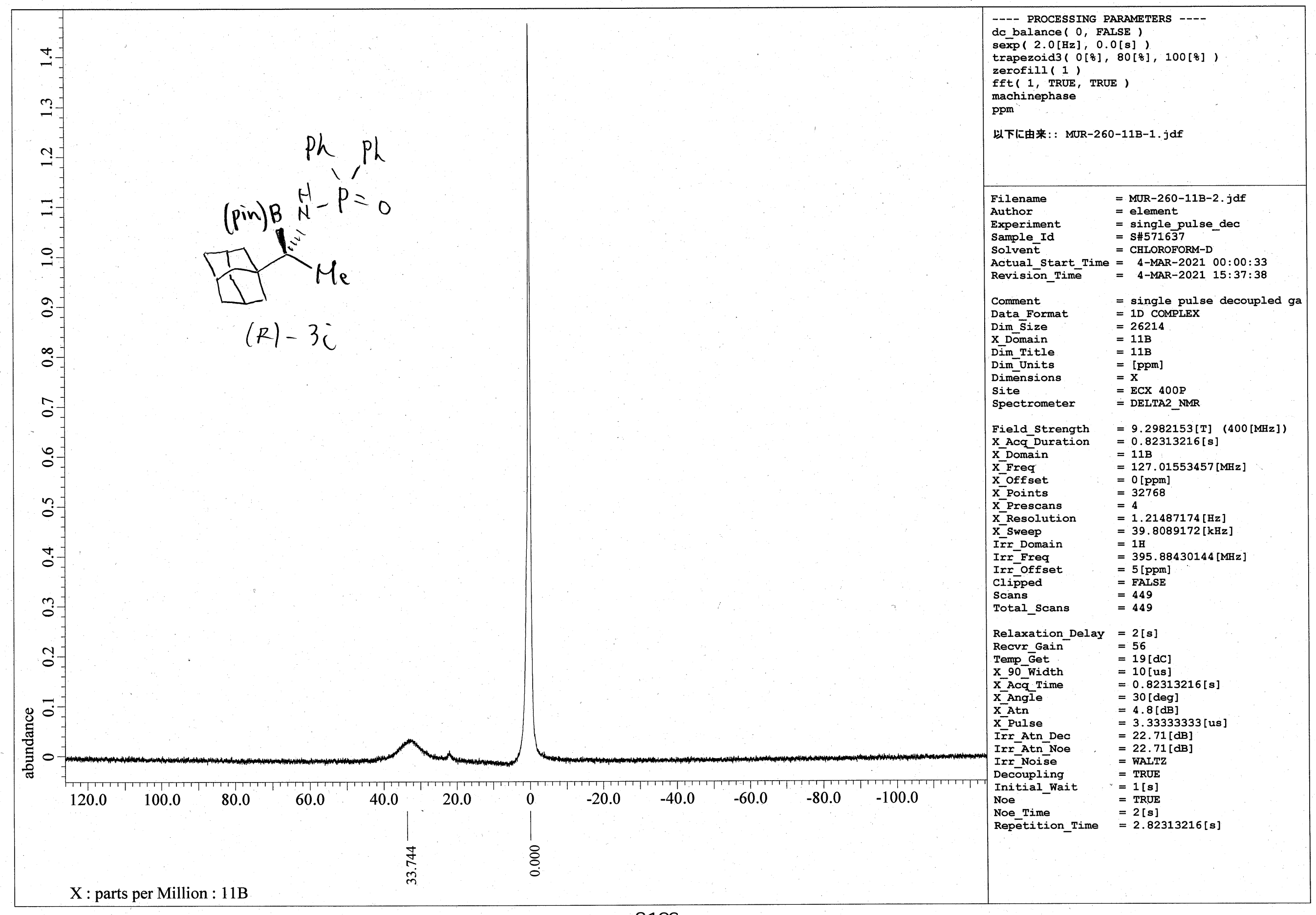




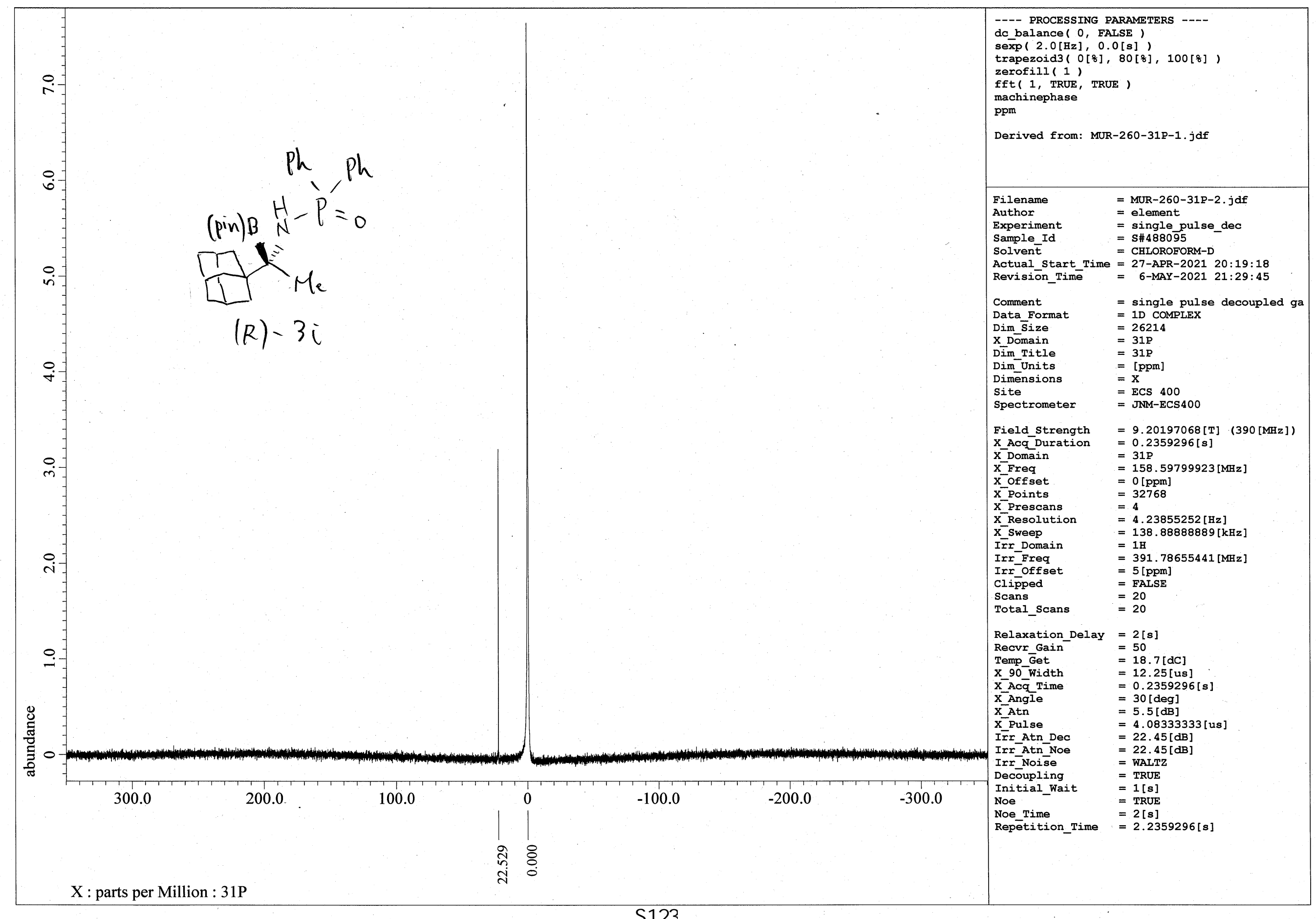




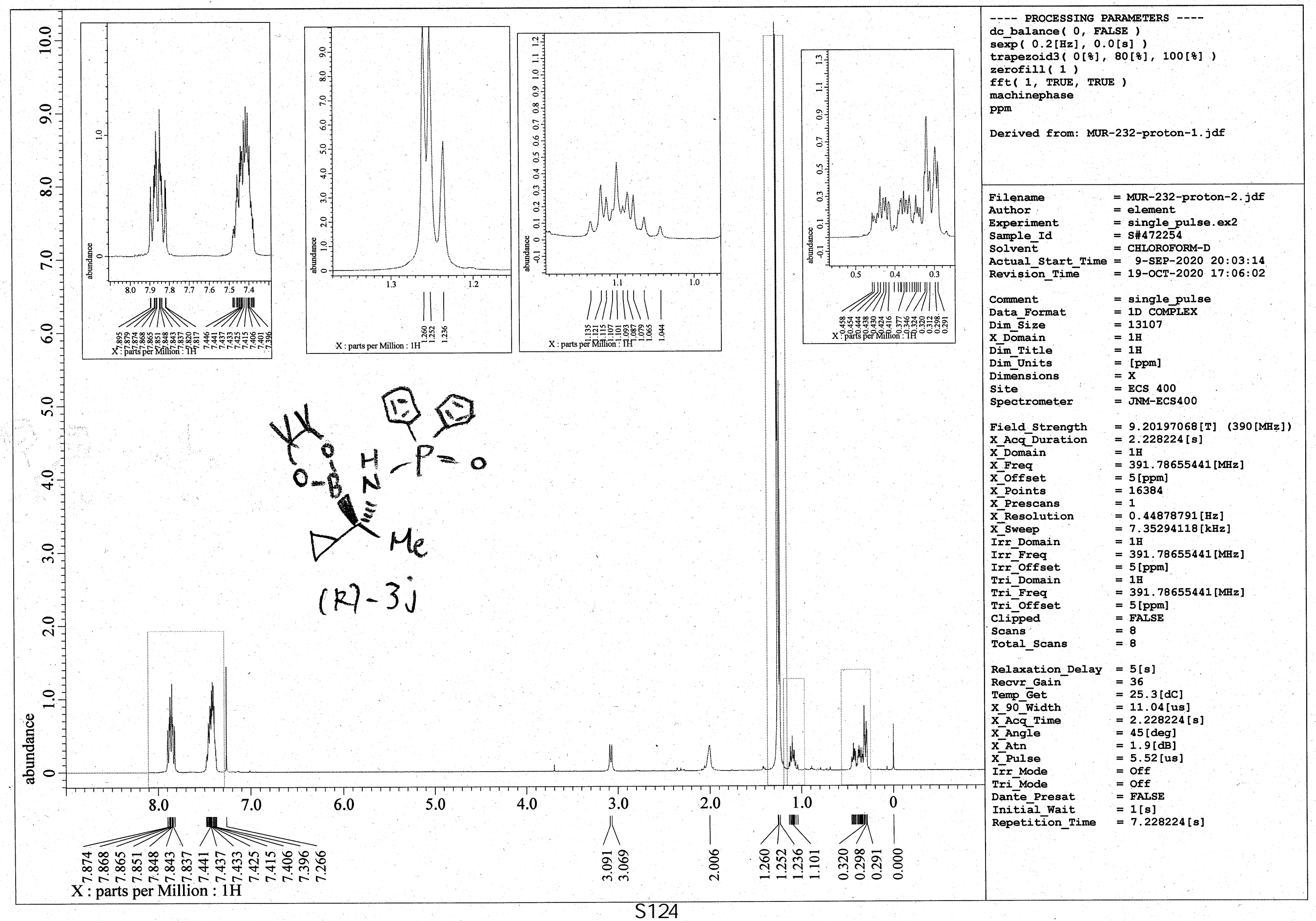




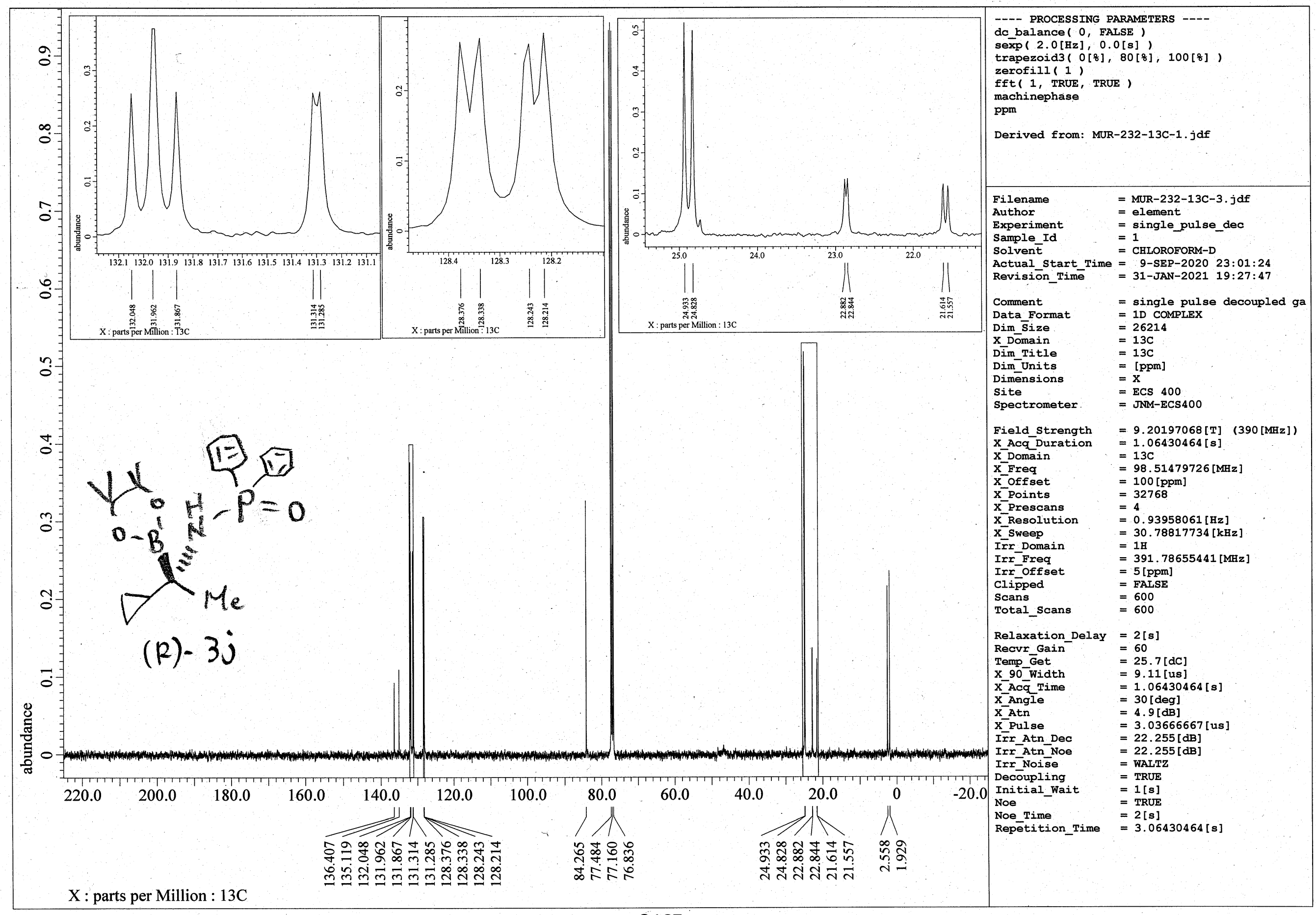




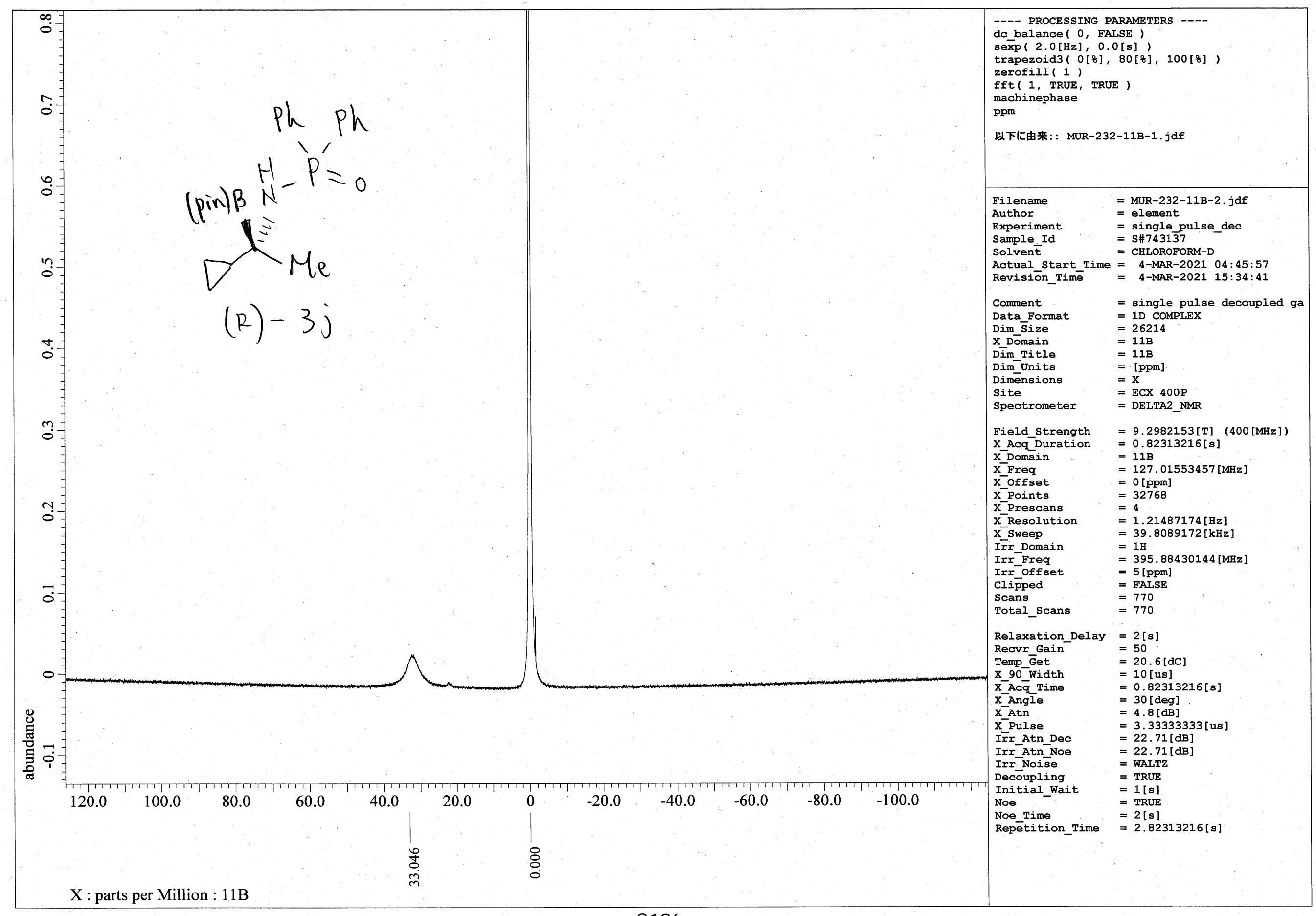




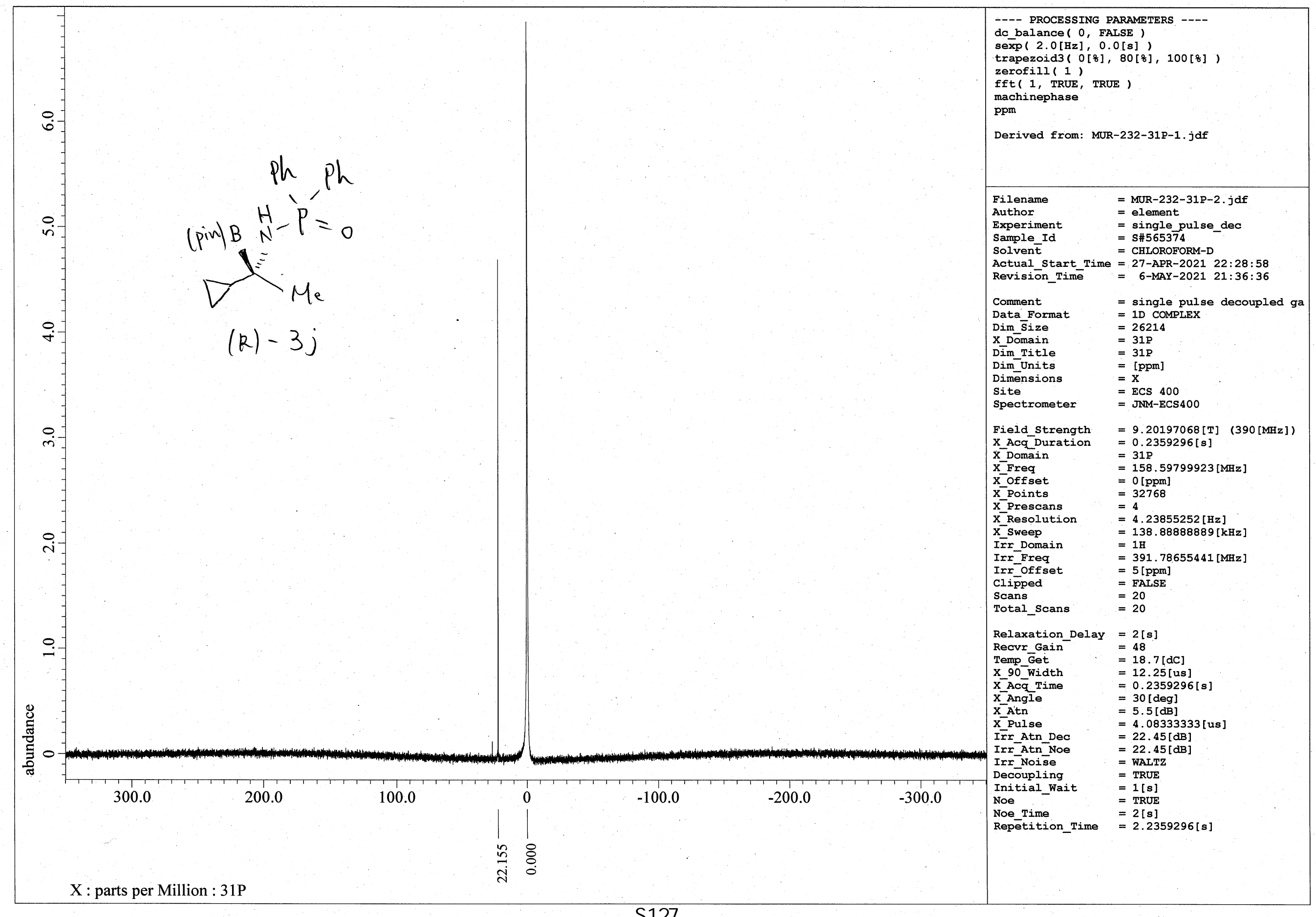




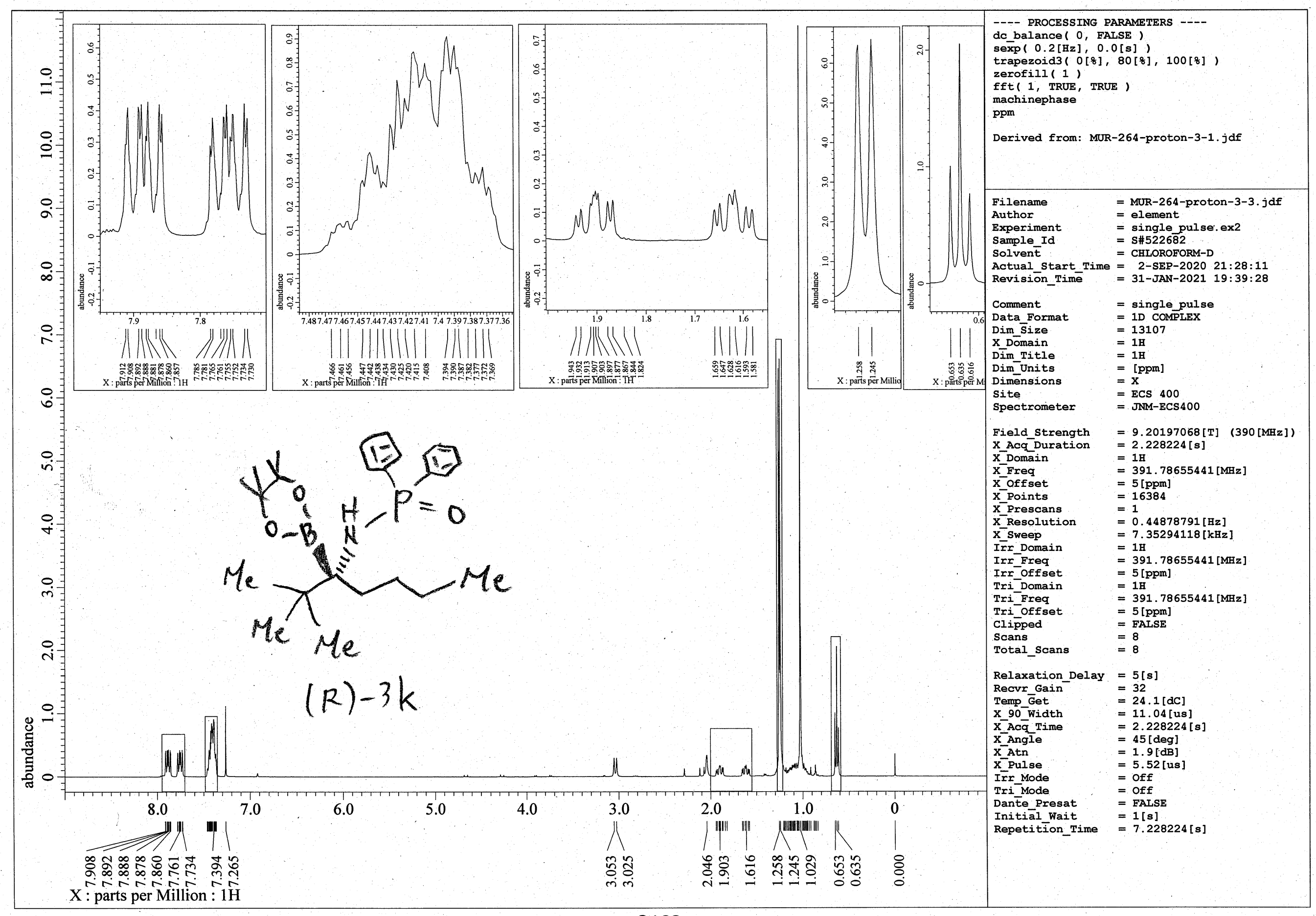




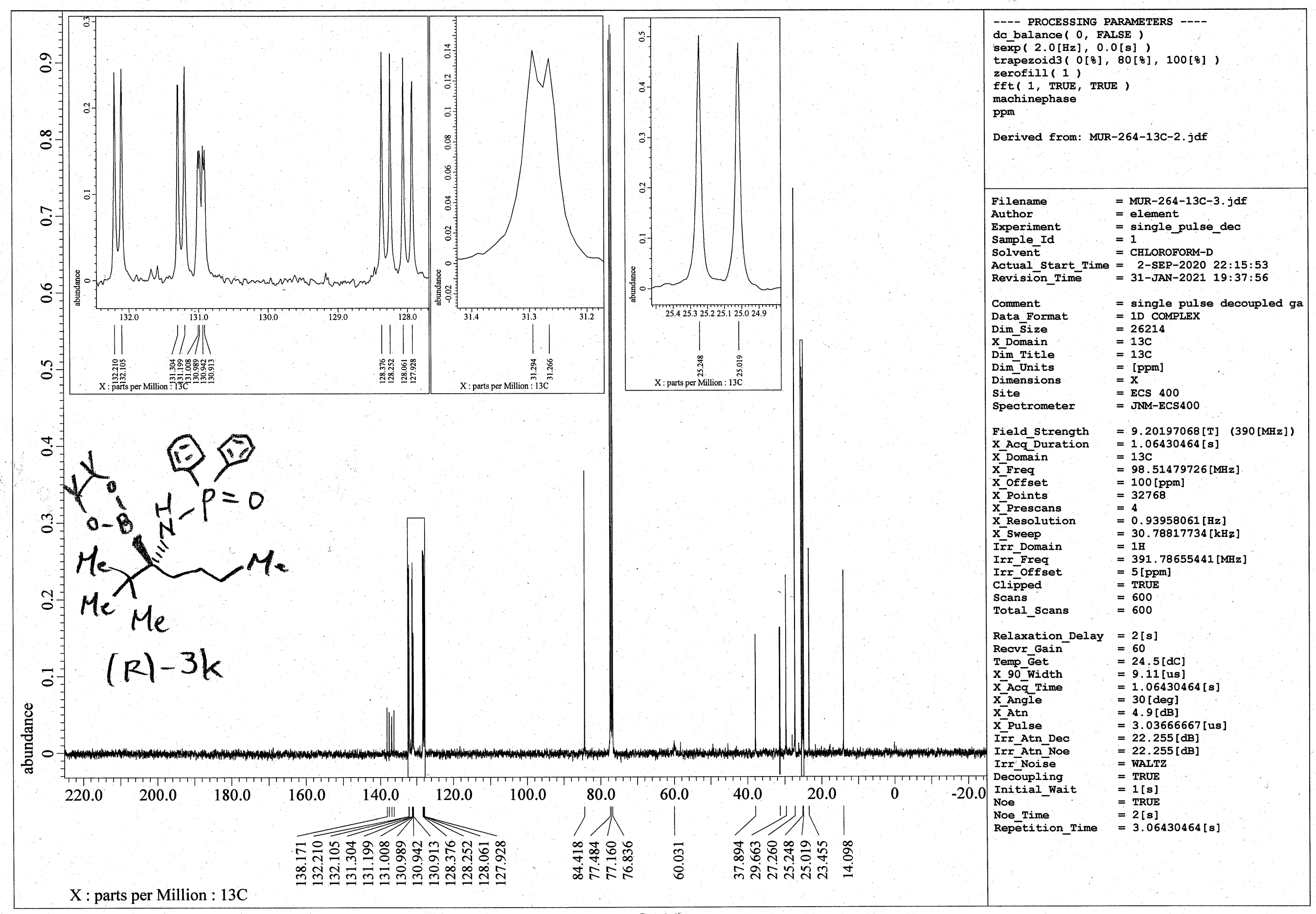




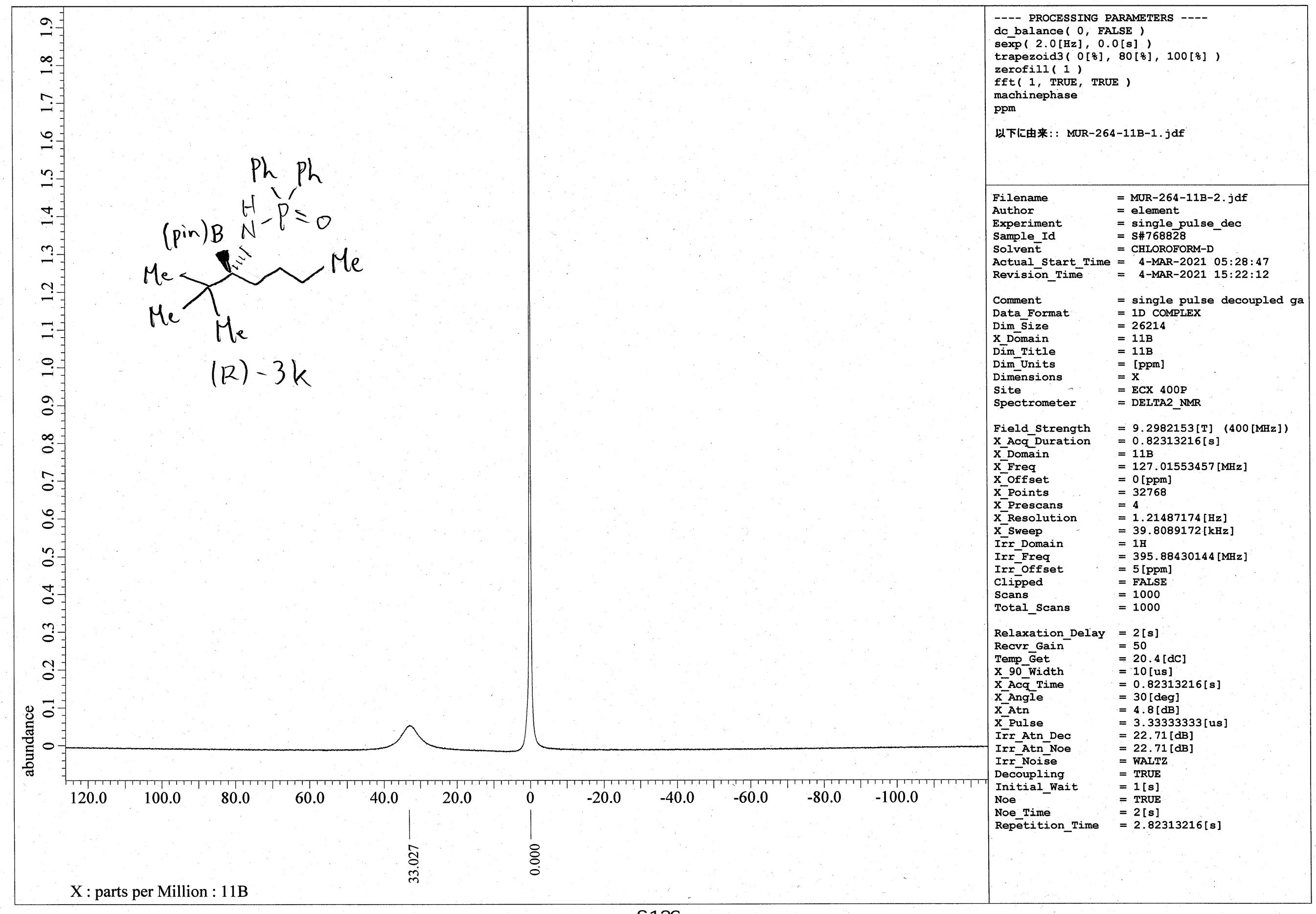




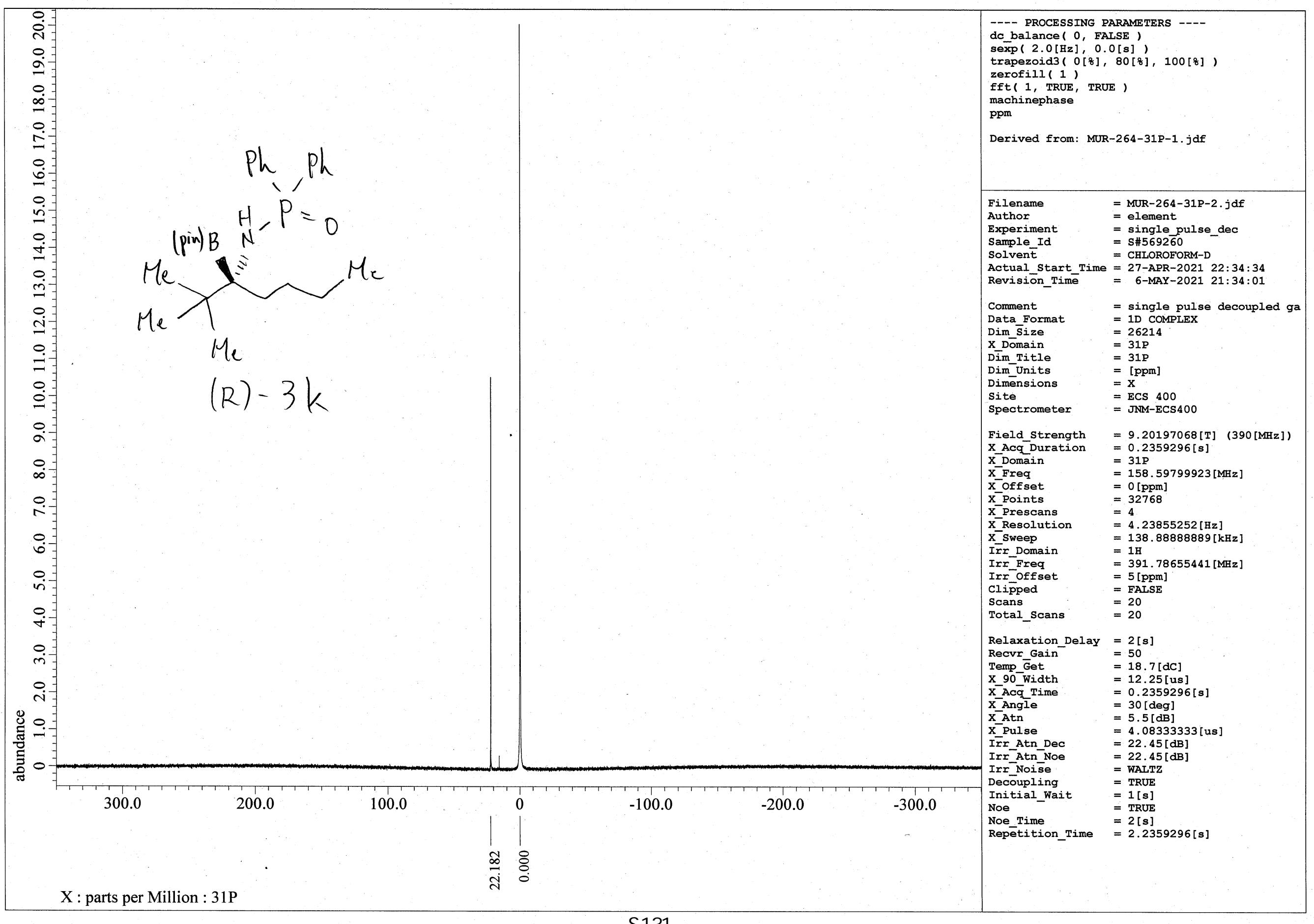




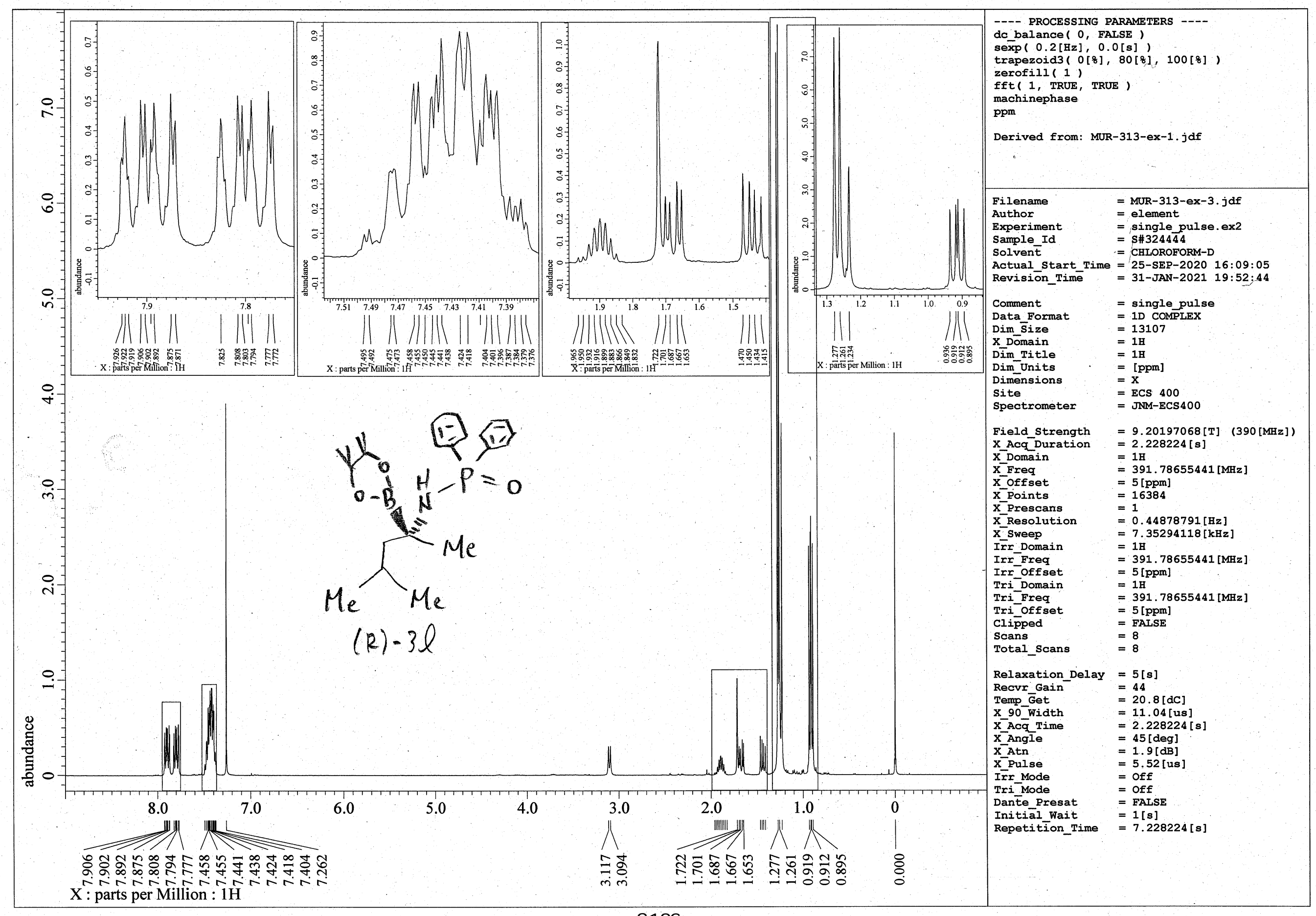




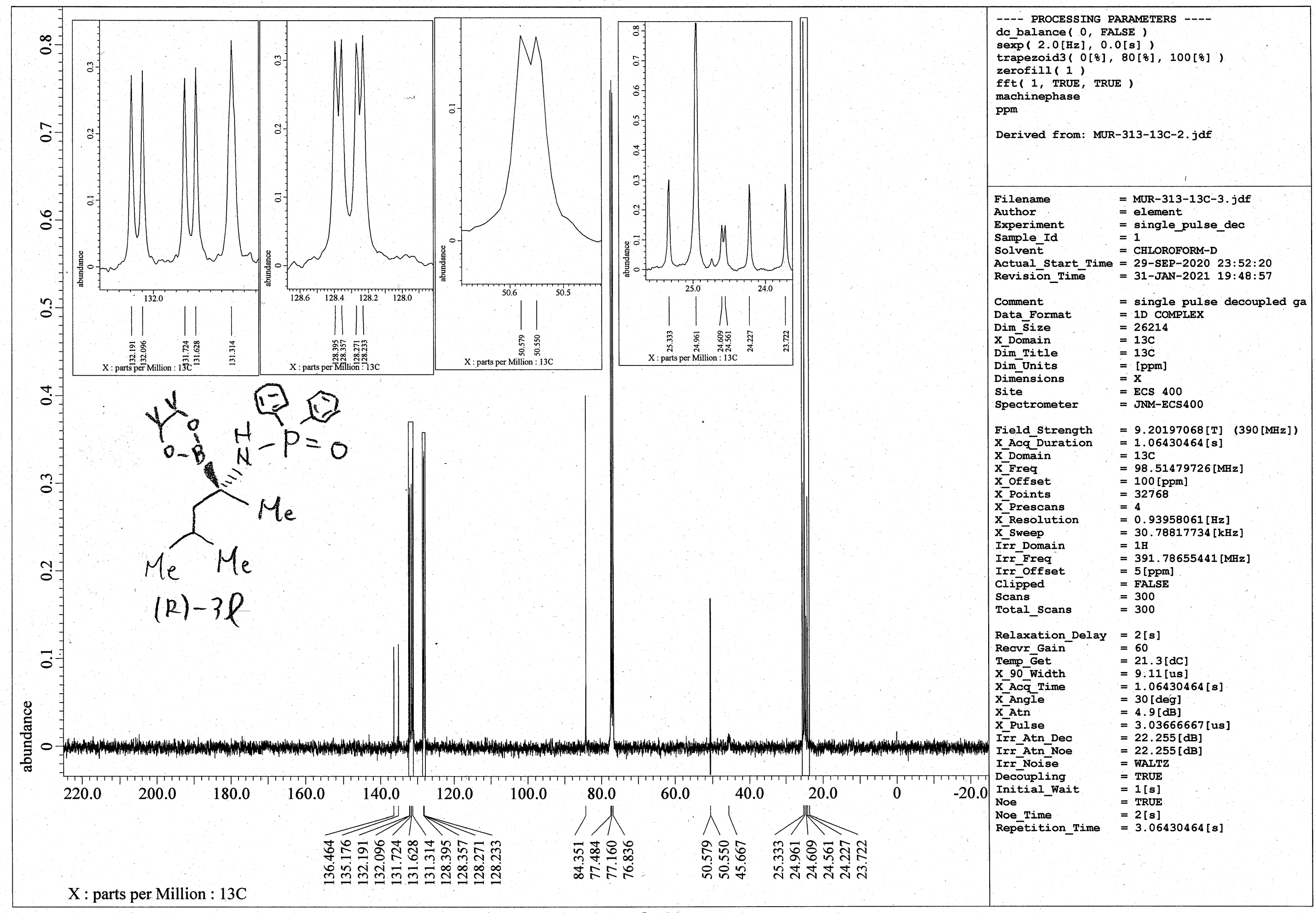




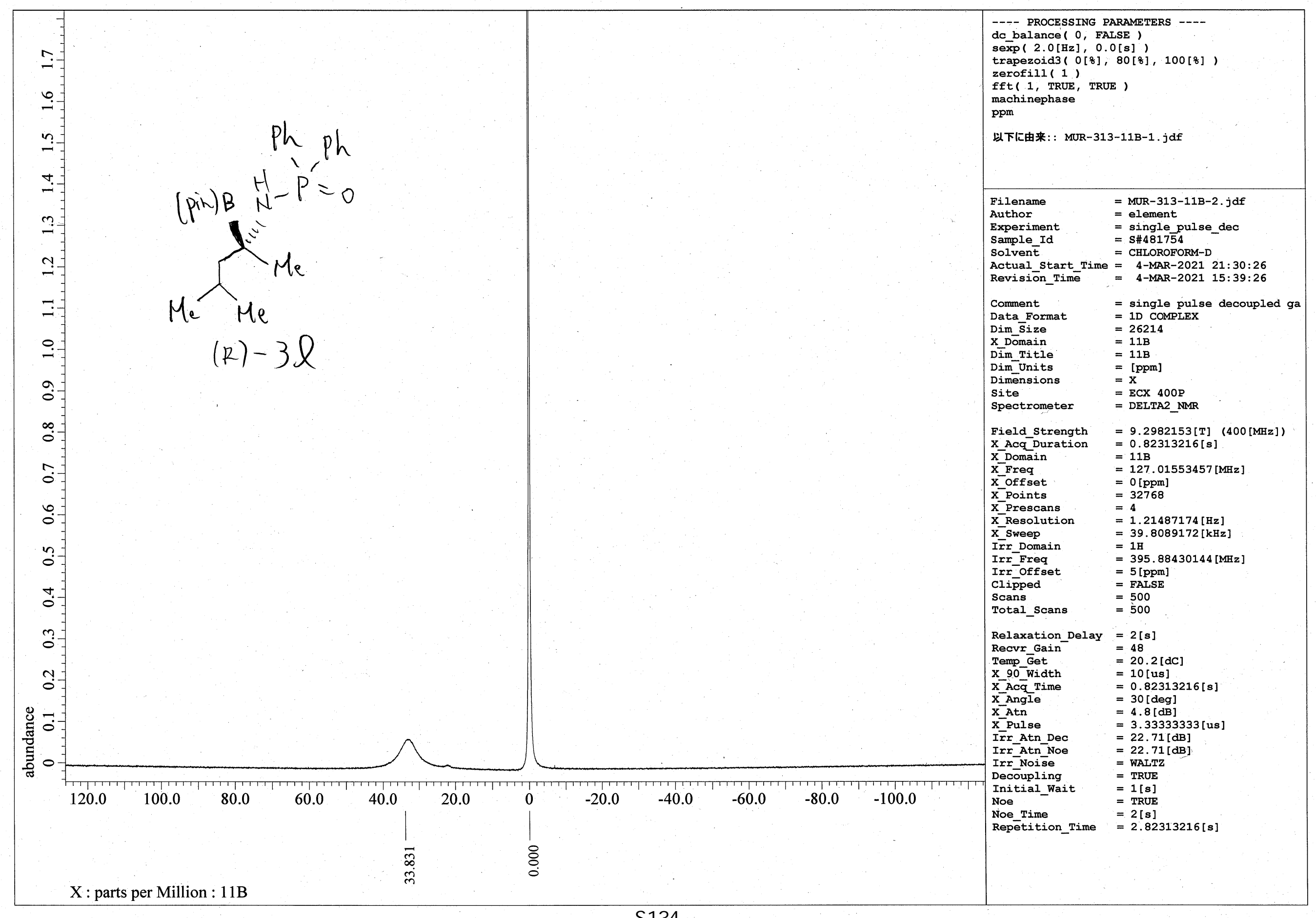




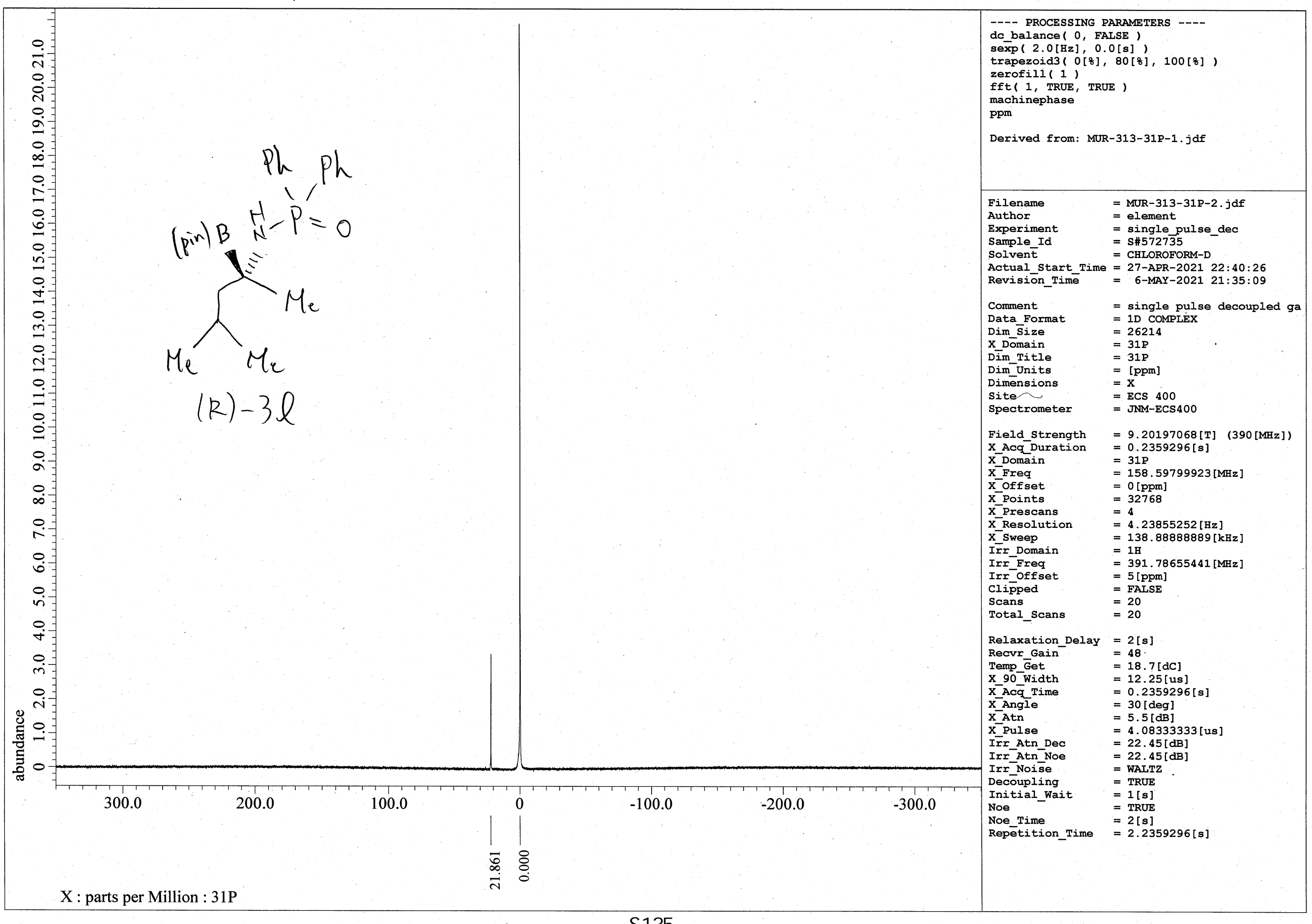




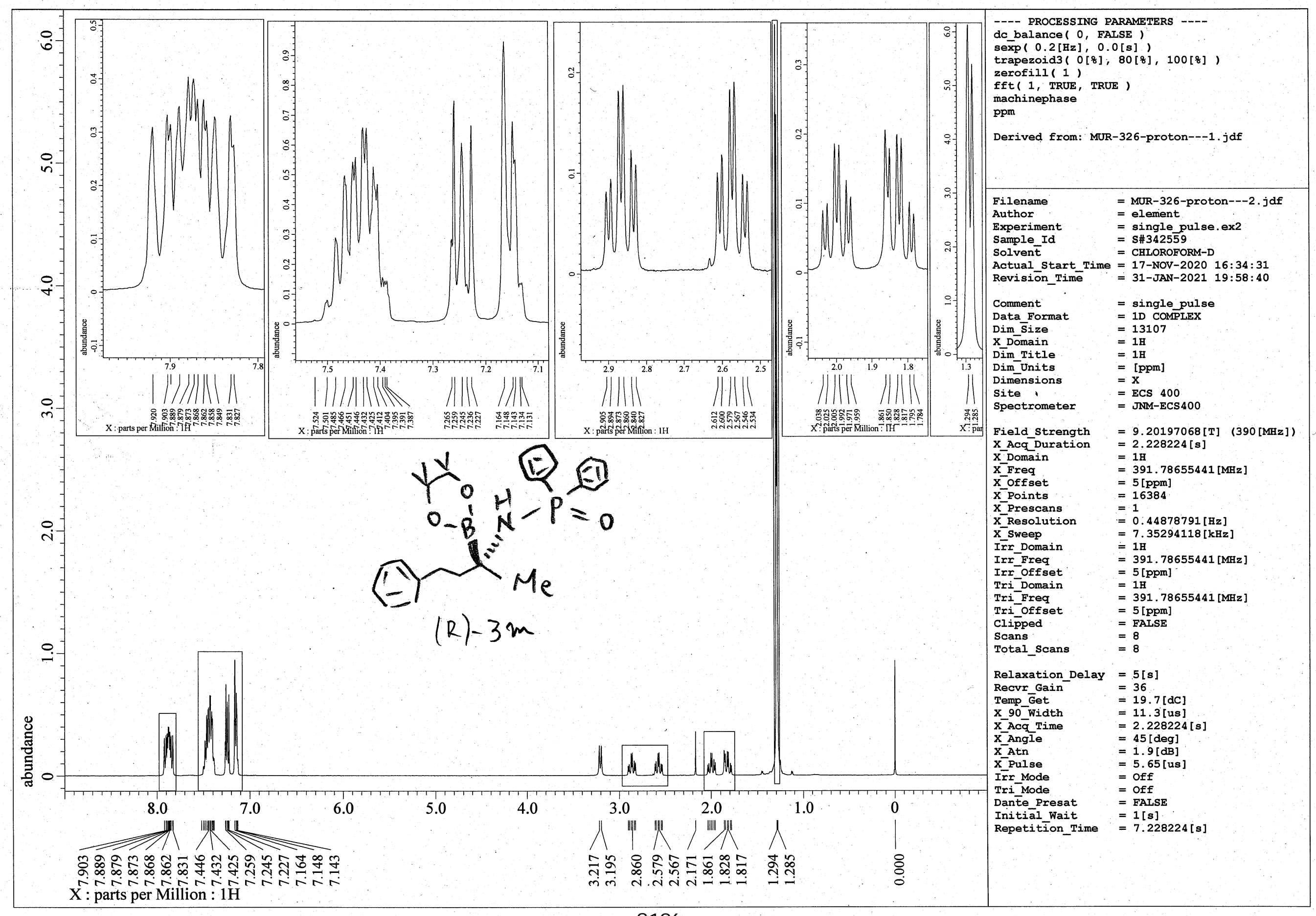




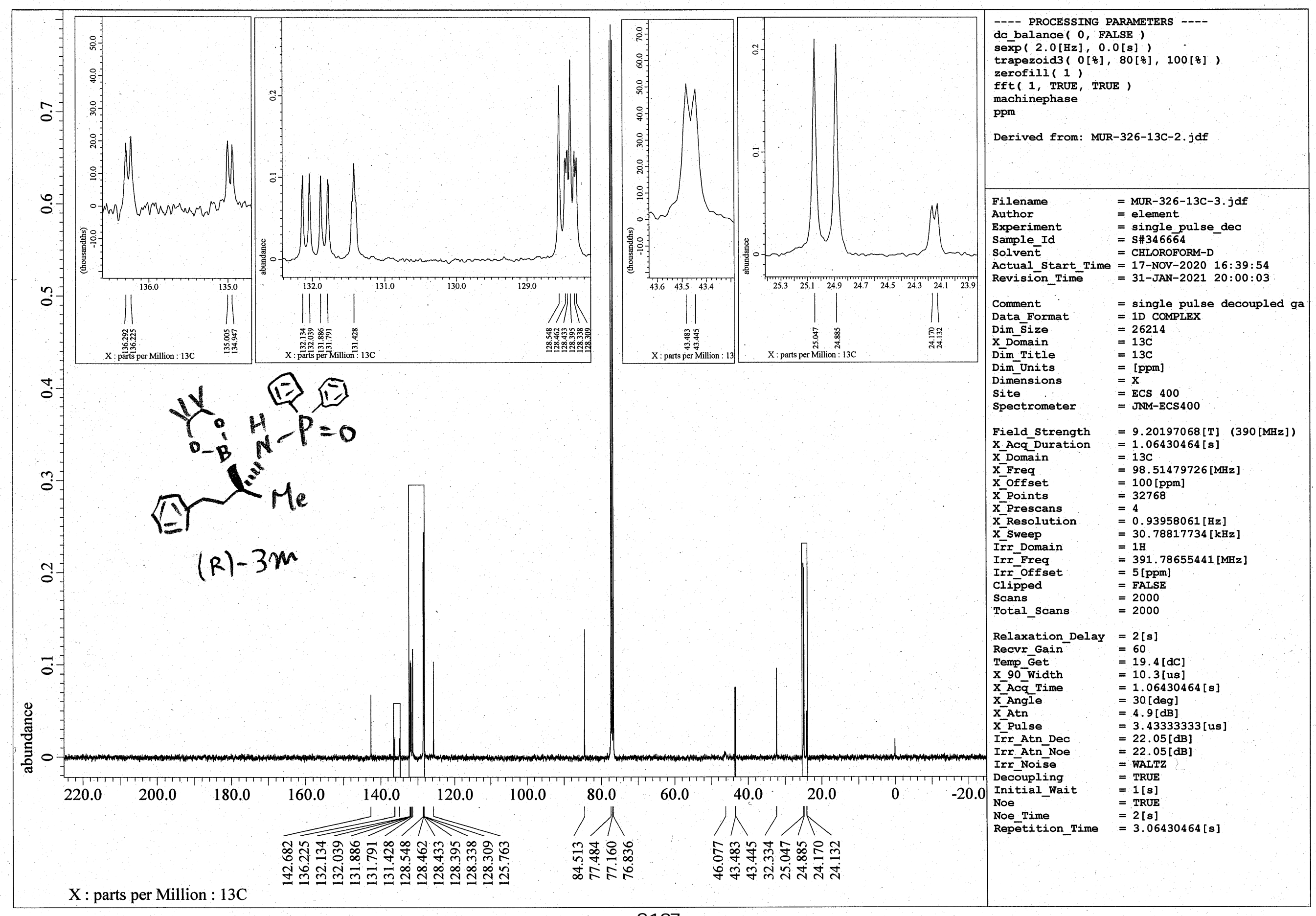




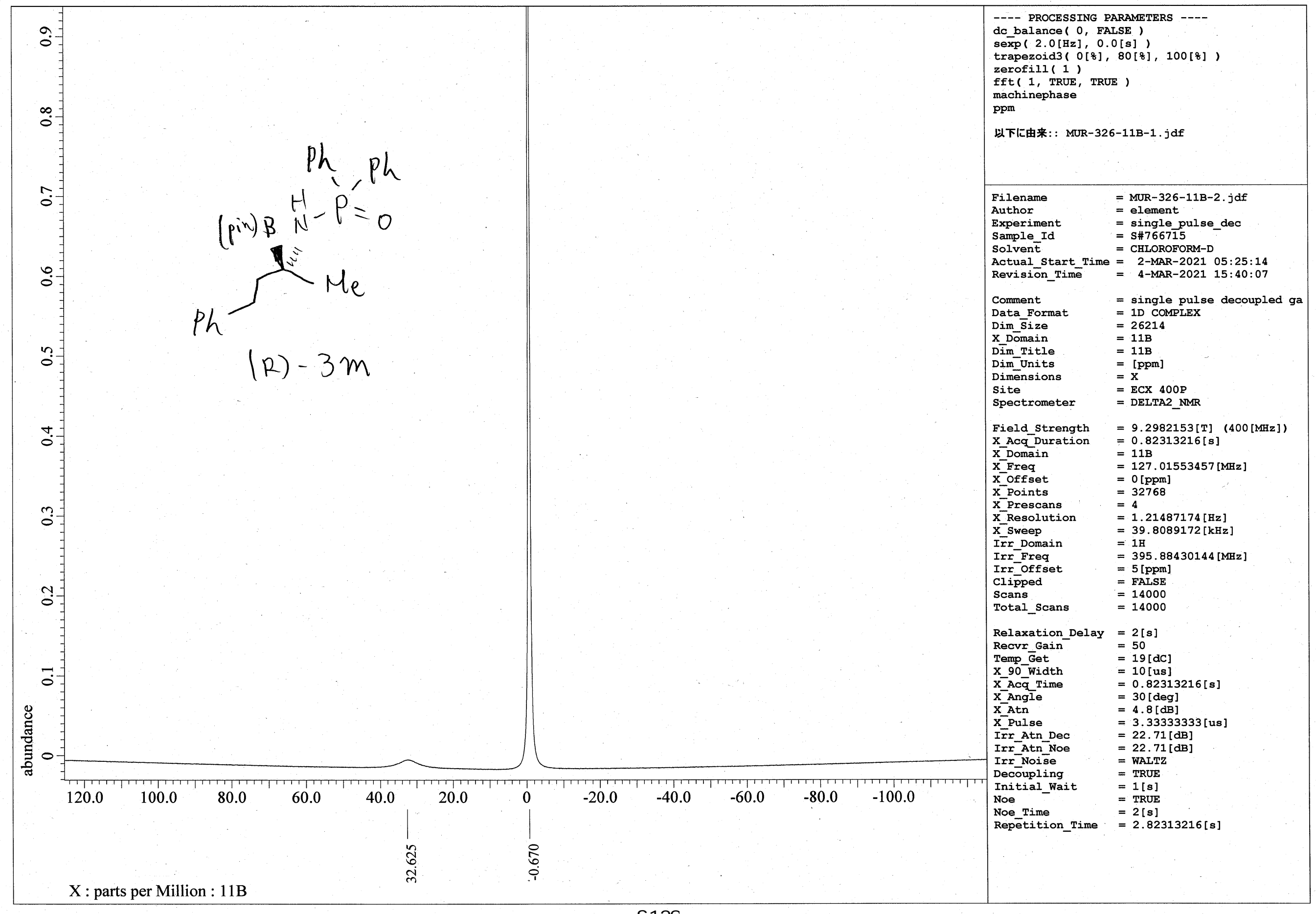




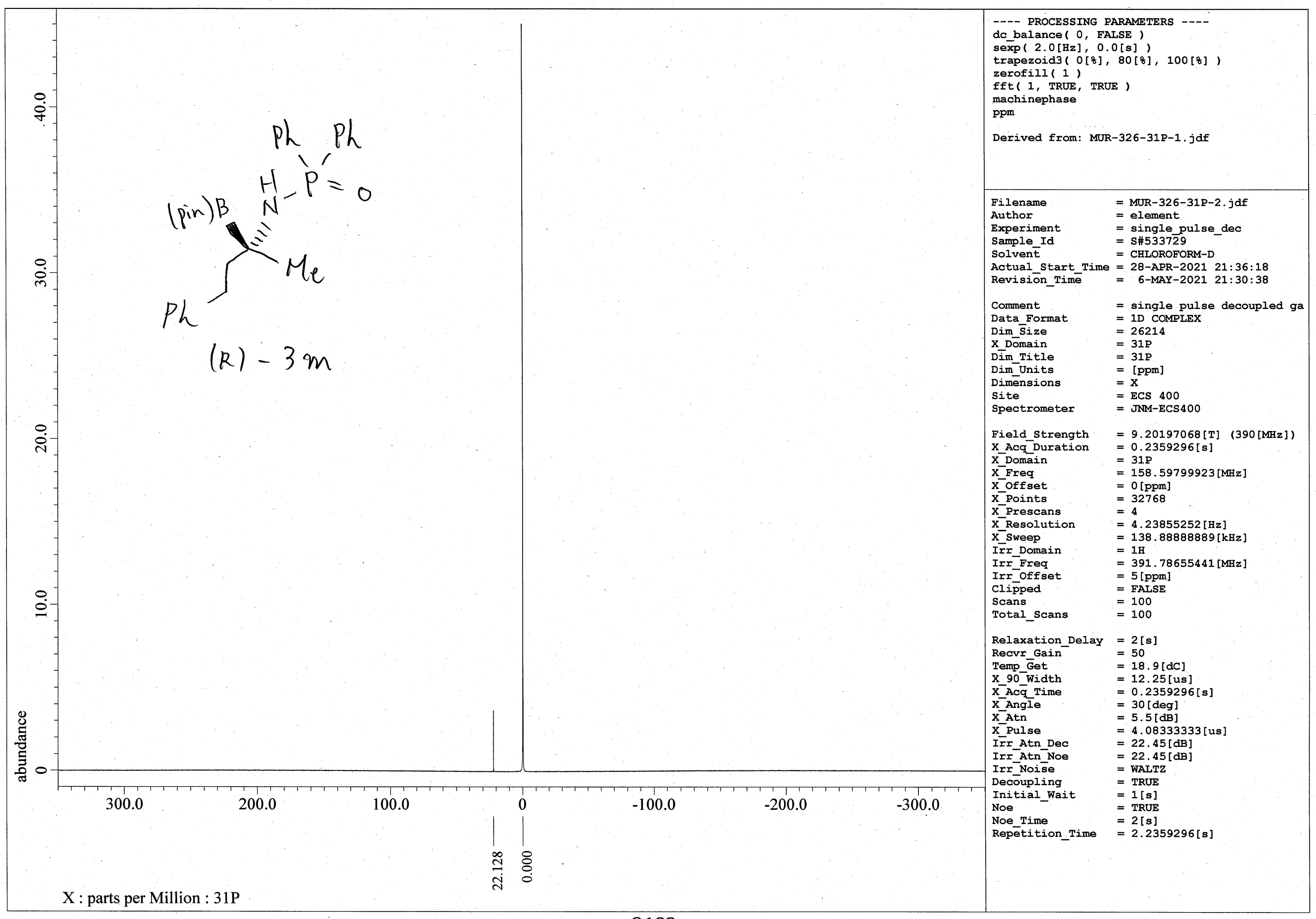




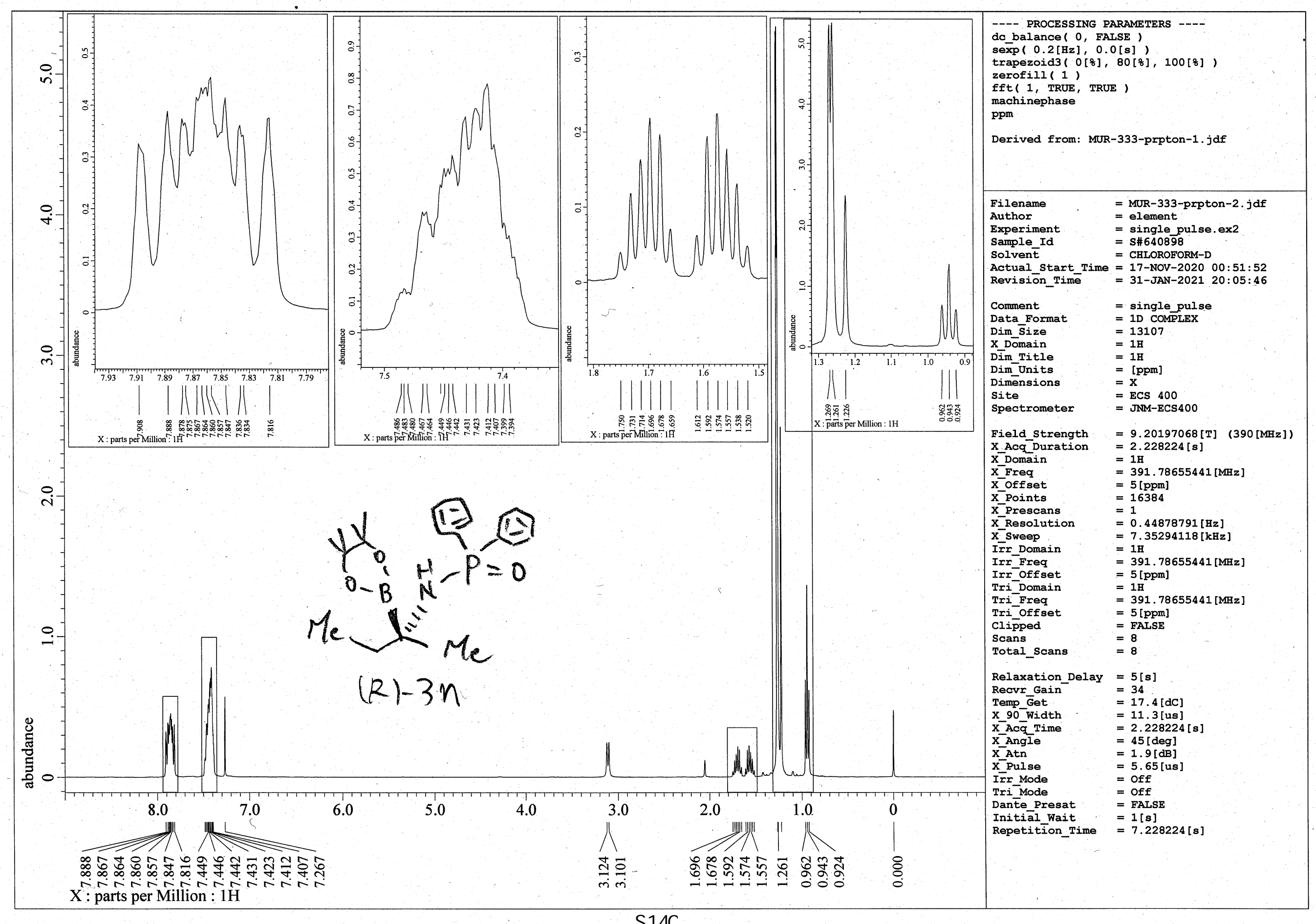




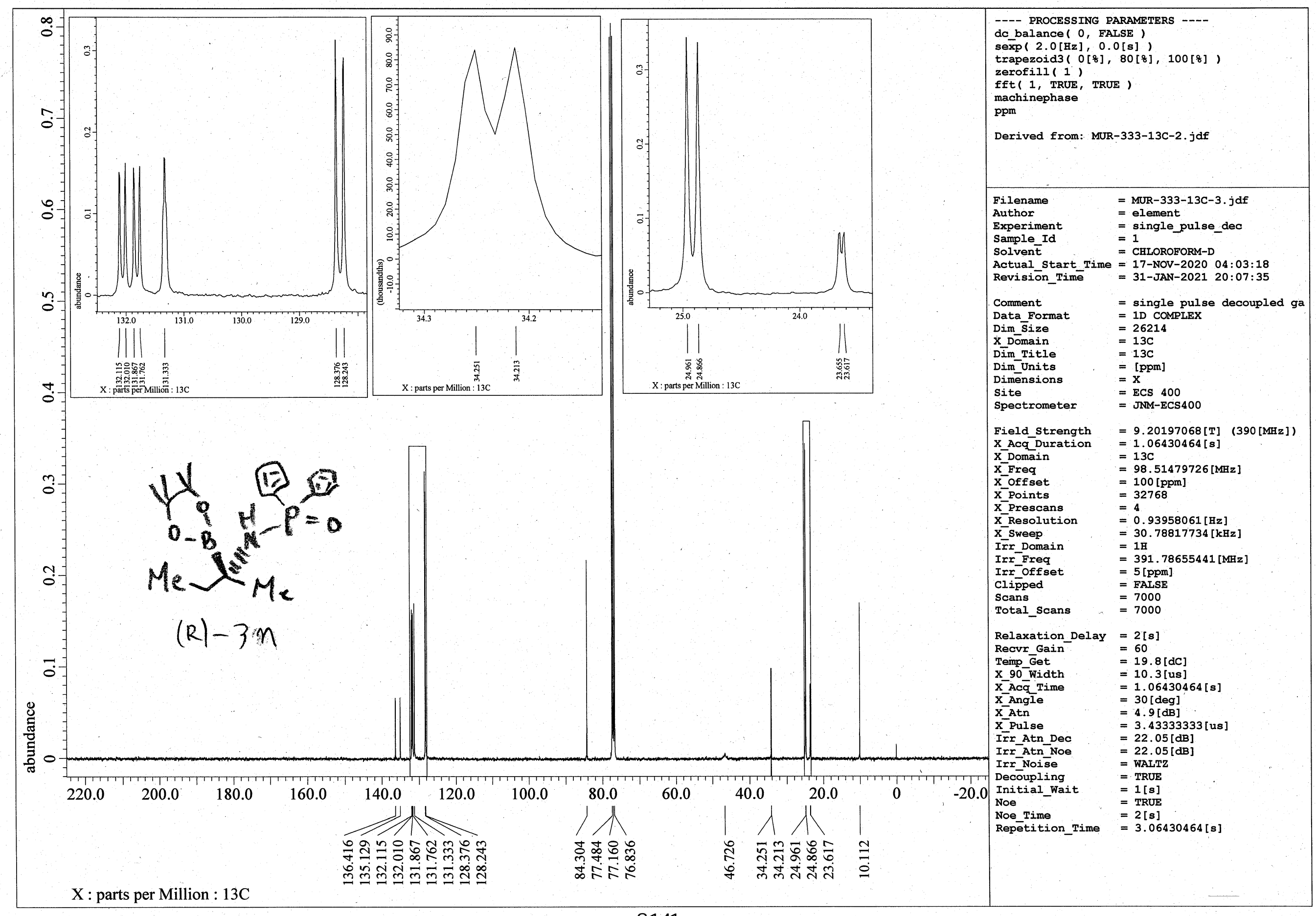




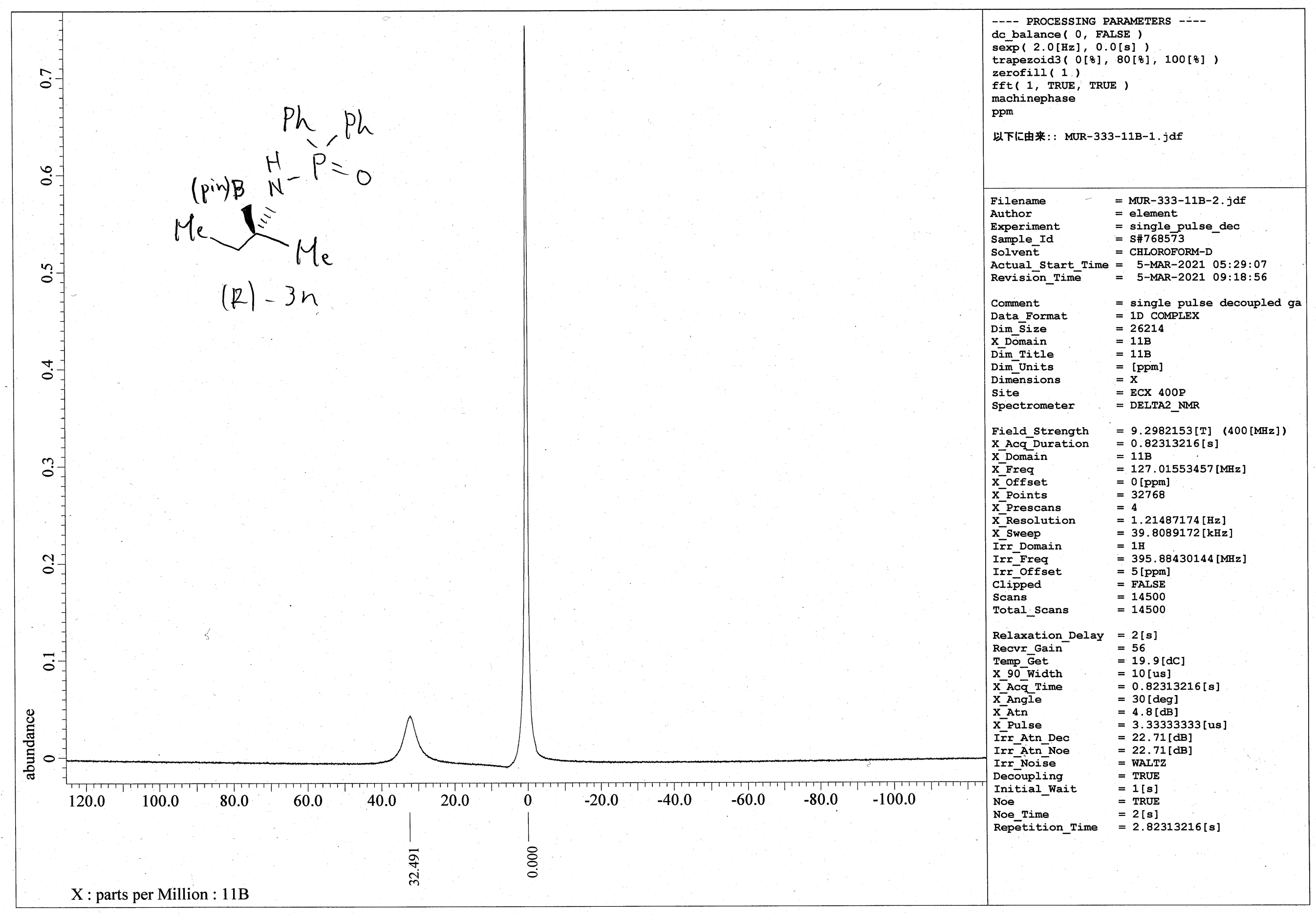




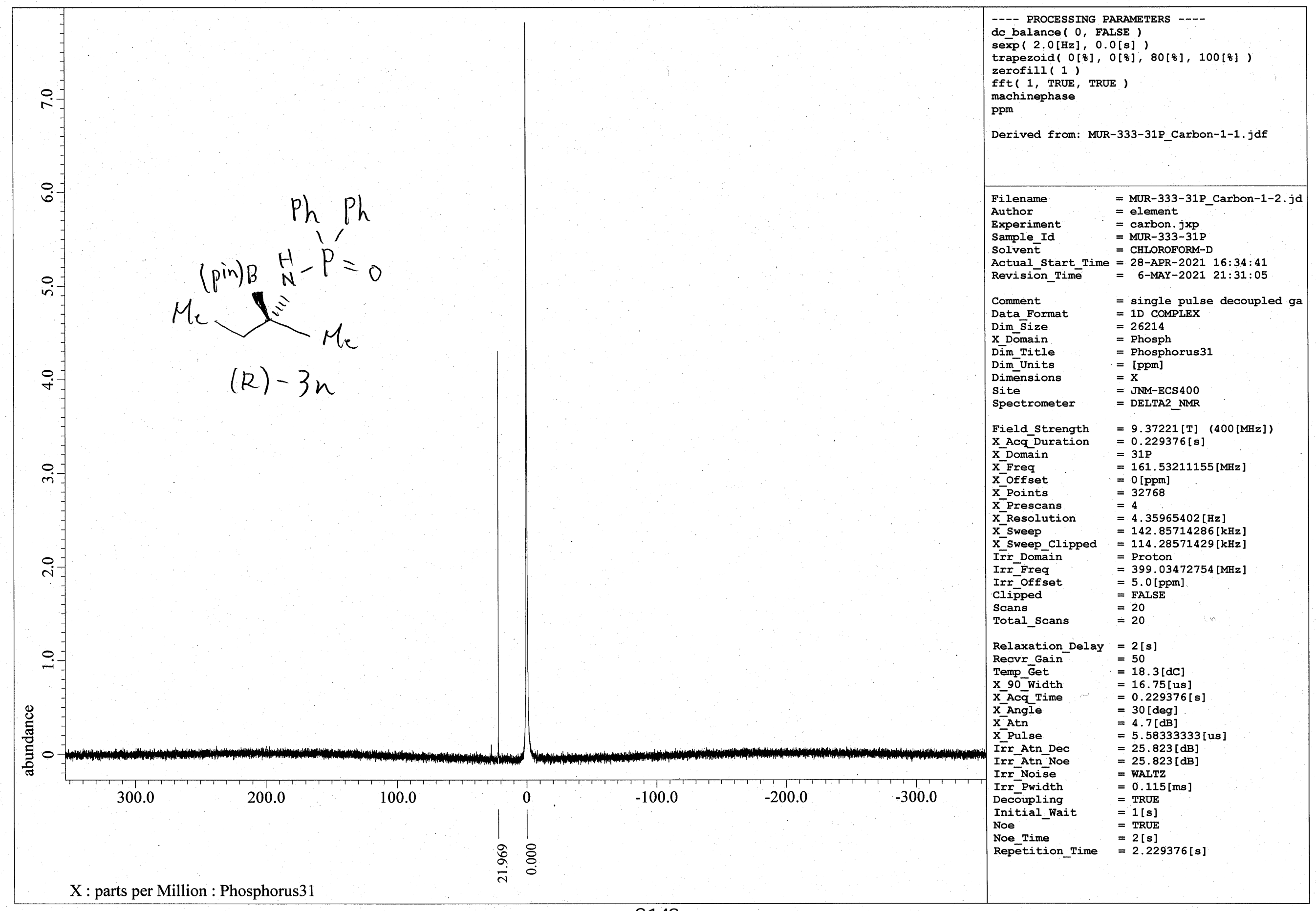




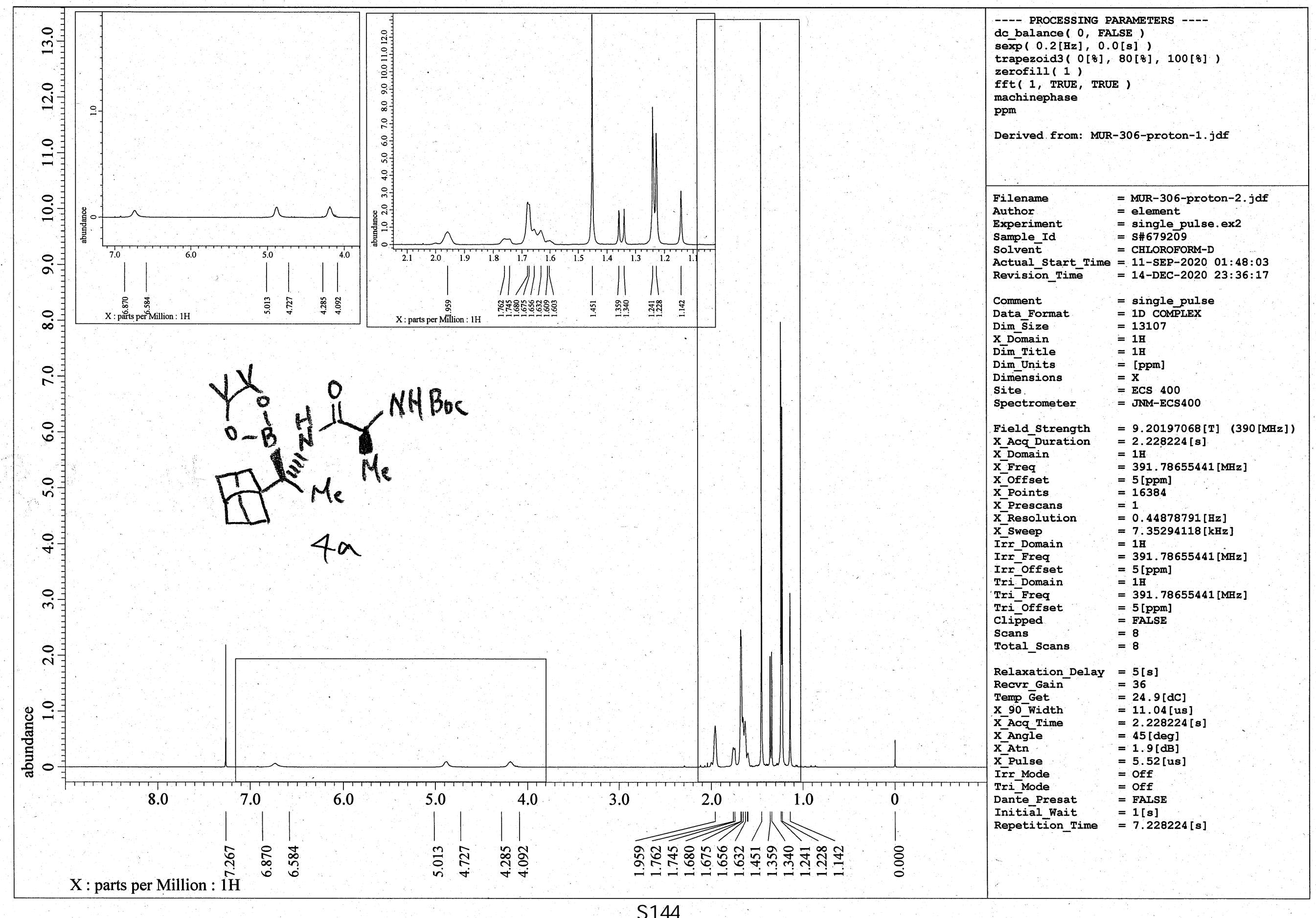




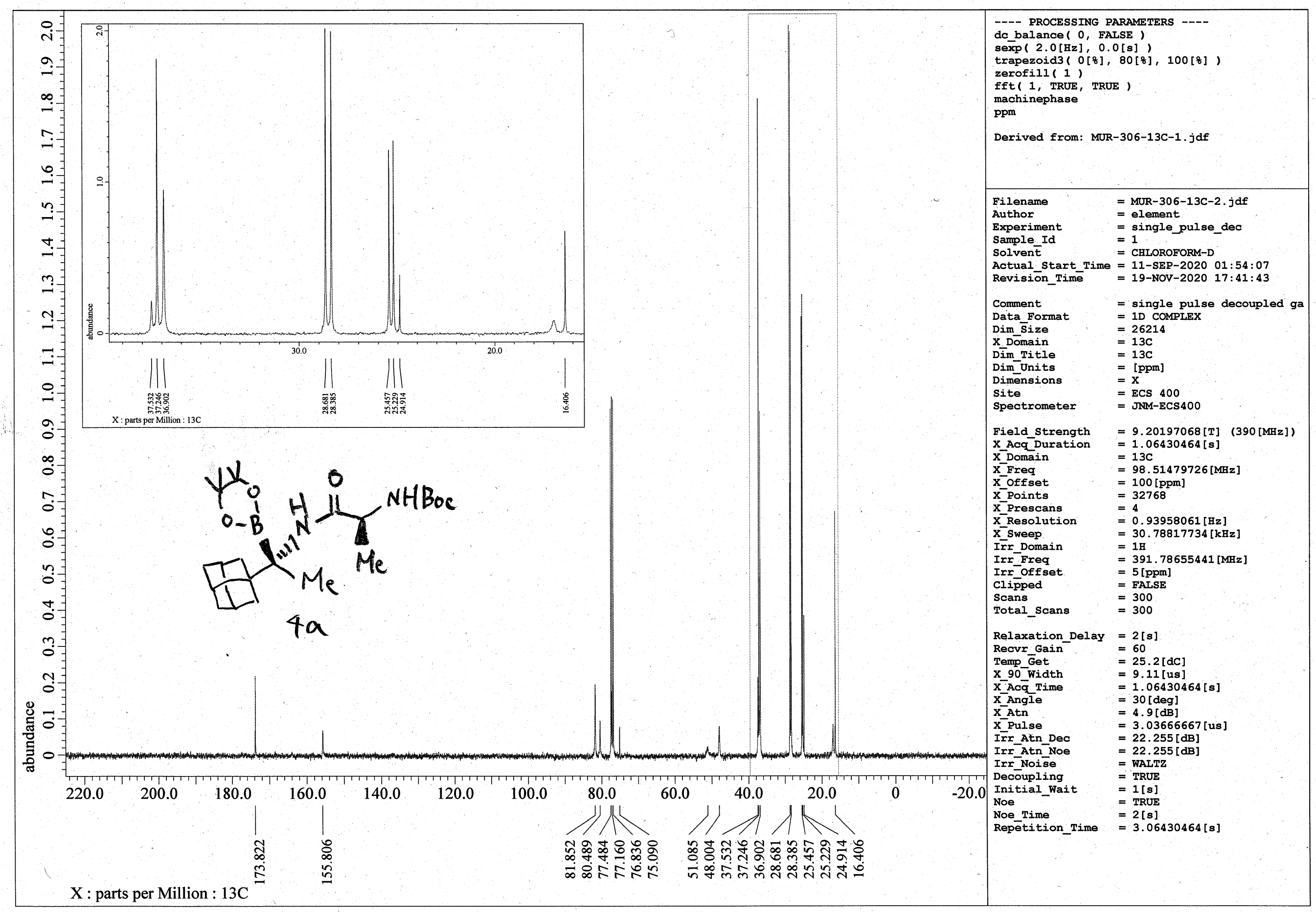




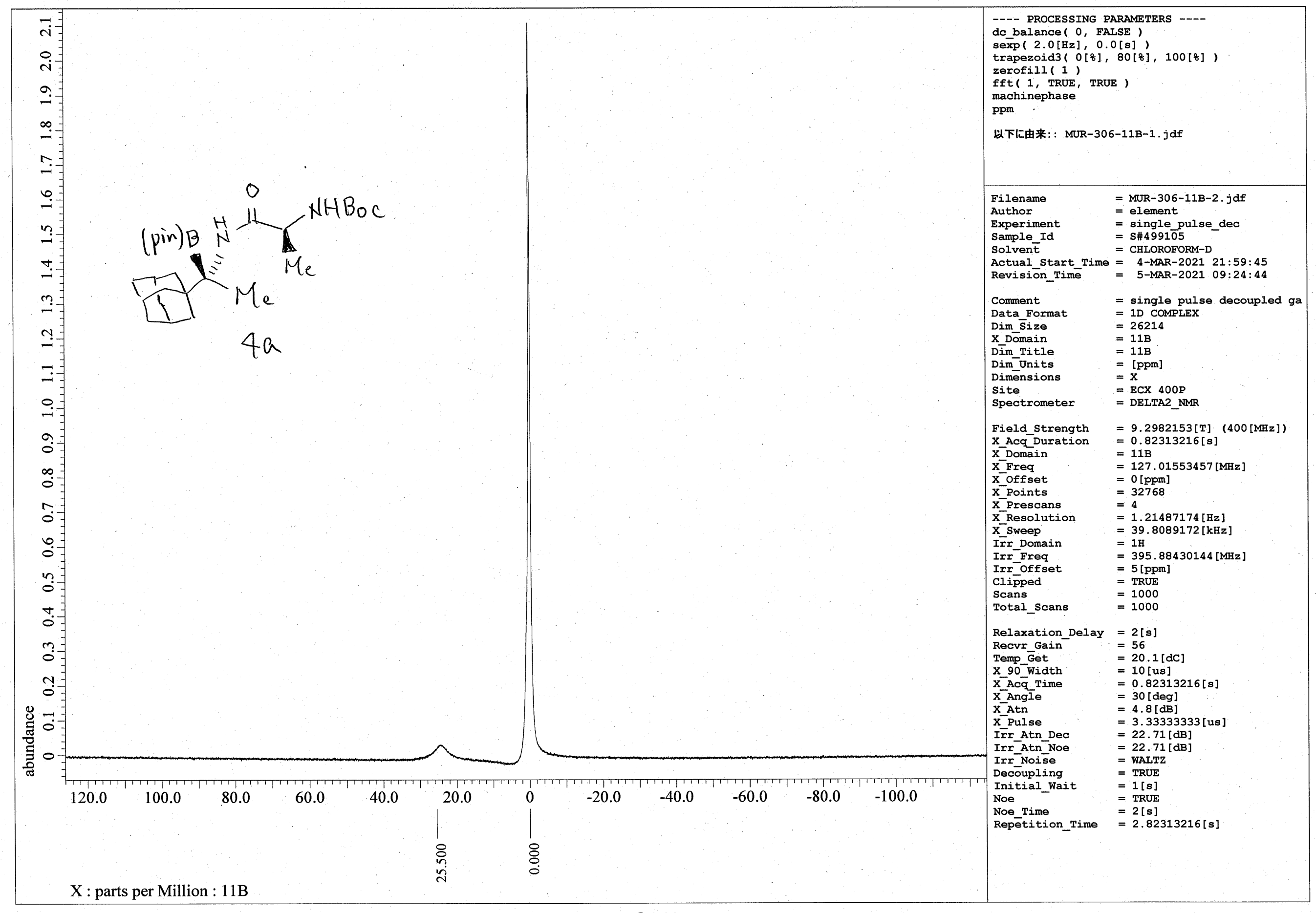




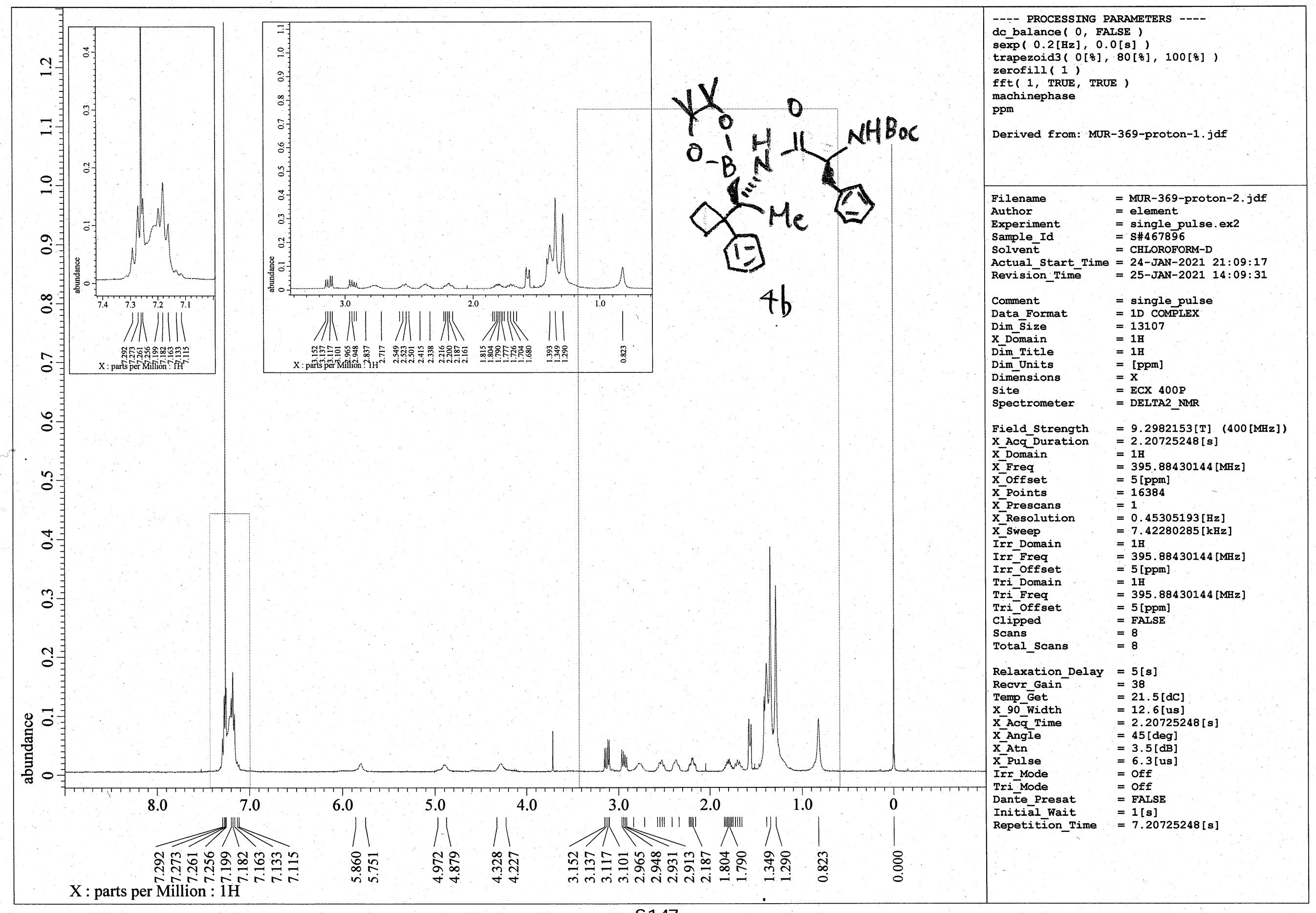




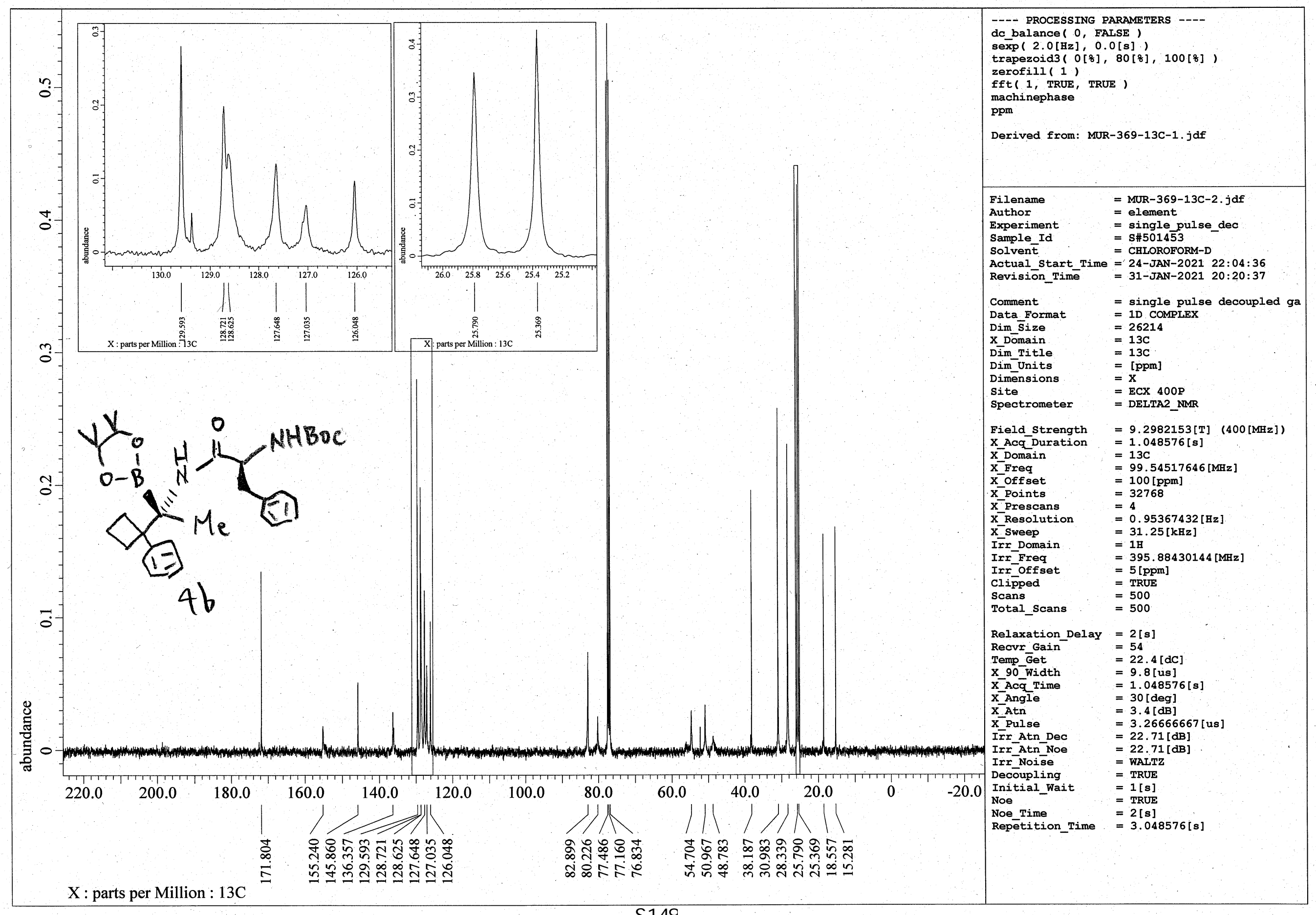




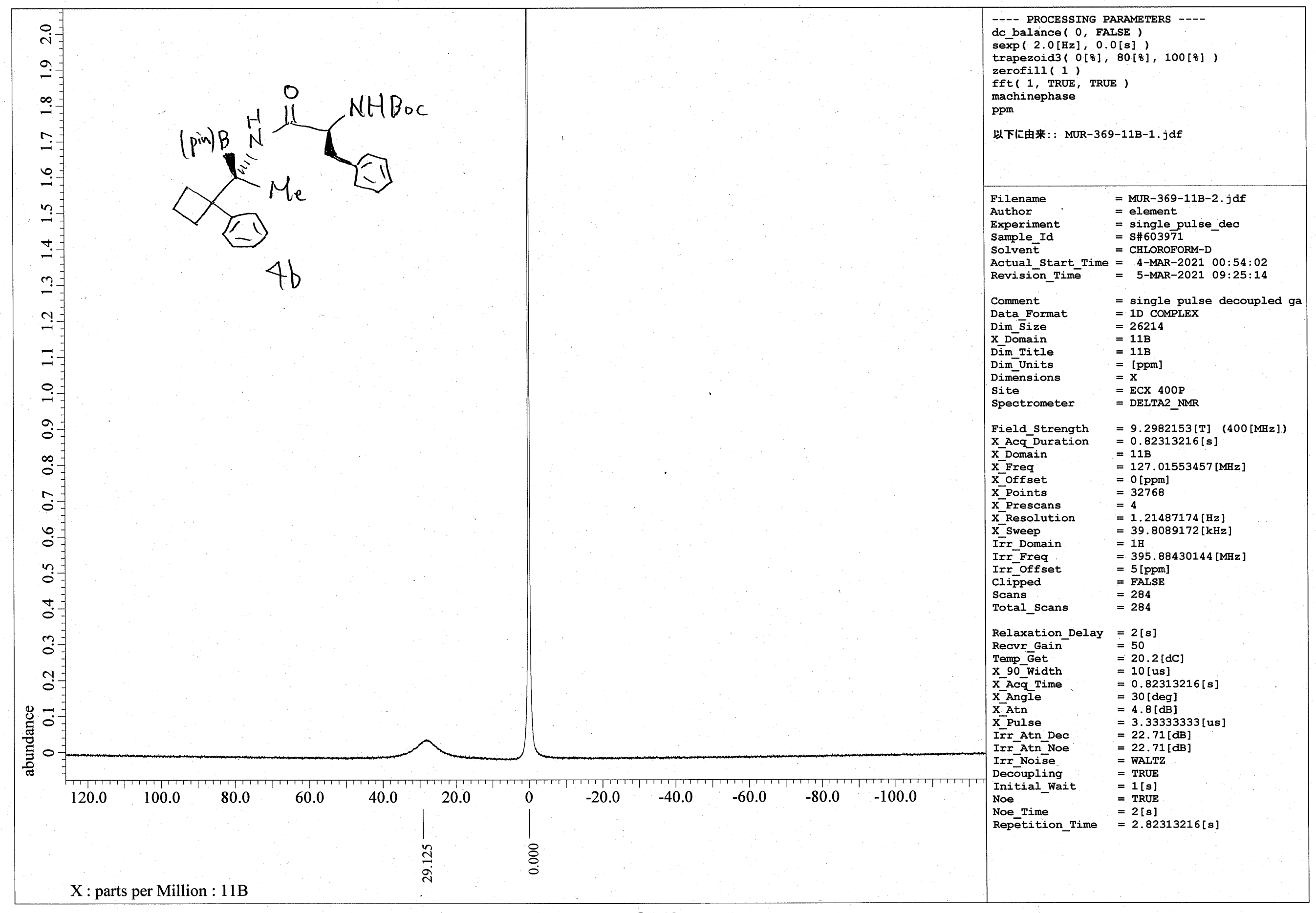




\section{D-2000 Elite HPLC System Manager Report}

Analyzed Date and Time: 2021/01/06

$$
16: 13
$$

Reported Date and Time: 2021/01/06

Processed Date and Time: 2021/01/06

$18: 55$

Data Path: C: \WIN32APP \D2000HSM \Isocratic \DATA \3131

Processing Method: 10/90 iPrOH/Hexane

System (acquisition): Sys 1

Application(data): Isocratic HPLC

Sample Name: MUR-363-OZ3-10\%

Series: 3131

Vial Number: 142

Vial Type: UNK

Injection from this vial: 1 of 1

Sample Description:

Volume: $10.0 \mathrm{ul}$

Chrom Type: HPLC Channel : 1

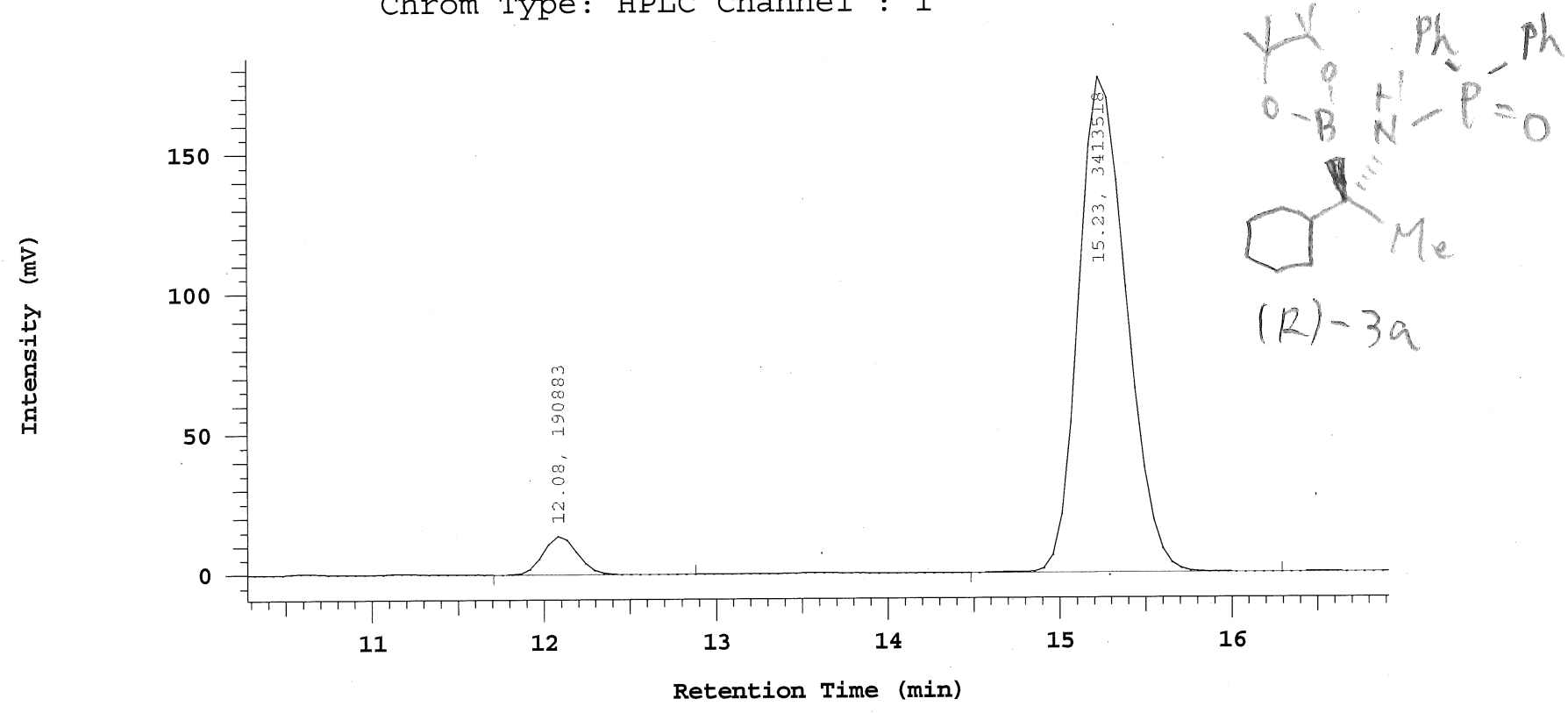

Processing Method: 10/90 iPrOH/Hexane

Column Type: OD-H 2

Method Developer: Administrator

Pump A: L-2130

Pump A Solvent A: Hexane

Pump A Solvent B: 10/90 iProH/Hexane

Pump A Solvent C: iPrOH

Pump A Solvent D: iPrOH

Method Description:

Chrom Type: HPLC Channel : 1

Peak Quantitation: AREA

Calculation Method: AREA\%

\begin{tabular}{rrrr} 
No. & RT & Area & Area \% \\
\hline 1 & 12.08 & 190883 & 5.296 \\
2 & 15.23 & 3413518 & 94.704 \\
\hline & & 3604401 & 100.000 \\
\hline
\end{tabular}

Peak rejection level: 0 


\section{D-2000 Elite HPLC System Manager Report}

Analyzed Date and Time: 2021/01/06

$$
17: 10
$$

Reported Date and Time: 2021/01/06

Processed Date and Time: 2021/01/06

$$
18: 56
$$

Data Path: C: \WIN32APP \D2000HSM \Isocratic \DATA \3132\

Processing Method: 10/90 iPrOH/Hexane

System (acquisition): Sys 1

Application(data): Isocratic HPLC

Sample Name: MUR-3a-rac

Injection from this vial: 1 of 1

Sample Description:
Series: 3132

Vial Number: 143

Vial Type: UNK

Volume: $10.0 \mathrm{ul}$

Chrom Type: HPLC Channel : 1

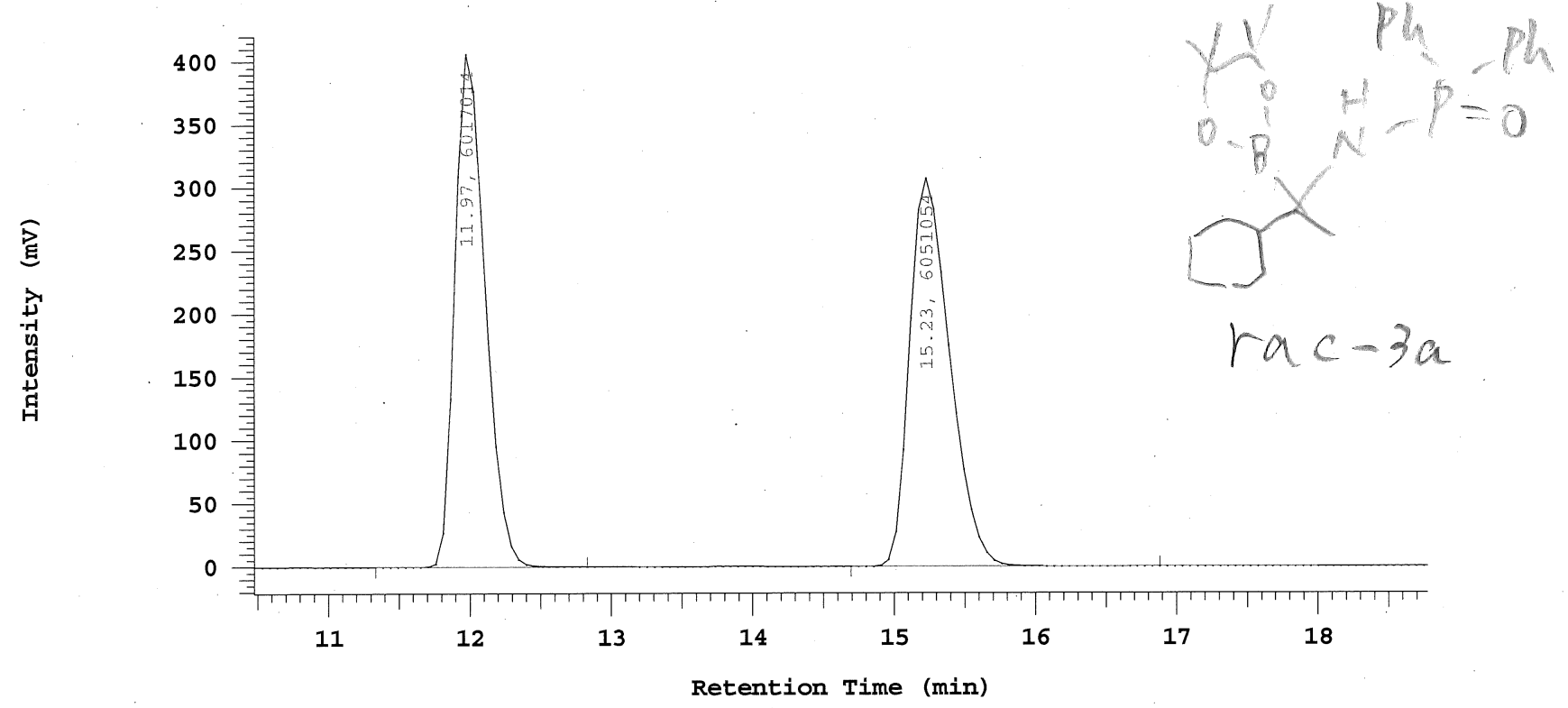

Processing Method: 10/90 iProH/Hexane Column Type: OD-H 2

Pump A: L-2130

Pump A Solvent A: Hexane

Pump A Solvent C: iProH

Method Developer: Administrator

Method Description:

Pump A Solvent B: 10/90 iProH/Hexane

Pump A Solvent D: iPrOH

Chrom Type: HPLC Channel : 1

Peak Quantitation: AREA

Calculation Method: AREA\%

\begin{tabular}{rrrr} 
No. & RT & Area & Area \\
\hline 1 & 11.97 & 6017014 & 49.859 \\
2 & 15.23 & 6051054 & 50.141 \\
\hline & & 12068068 & 100.000
\end{tabular}

Peak rejection level: 0 


\section{D-2000 Elite HPLC System Manager Report}

Analyzed Date and Time: 2020/04/15 $15: 22$

Reported Date and Time: $\begin{aligned} & 2020 / 04 / 15 \\ & 16: 08\end{aligned}$

Processed Date and Time: $\begin{aligned} & 2020 / 04 / 15 \\ & 16: 07\end{aligned}$

Data Path: C: \WIN32APP \D2000HSM \Isocratic \DATA \2970

Processing Method: 10/90 iPrOH/Hexane

System (acquisition): Sys 1

Application(data): Isocratic HPLC

Sample Name: MUR-210-L22-OZ3-10\%

Injection from this vial: 1 of 1

Sample Description:
Series: 2970

Vial Number: 145

Vial Type: UNK

Volume: $10.0 \mathrm{ul}$

Chrom Type: HPLC Channel : 1

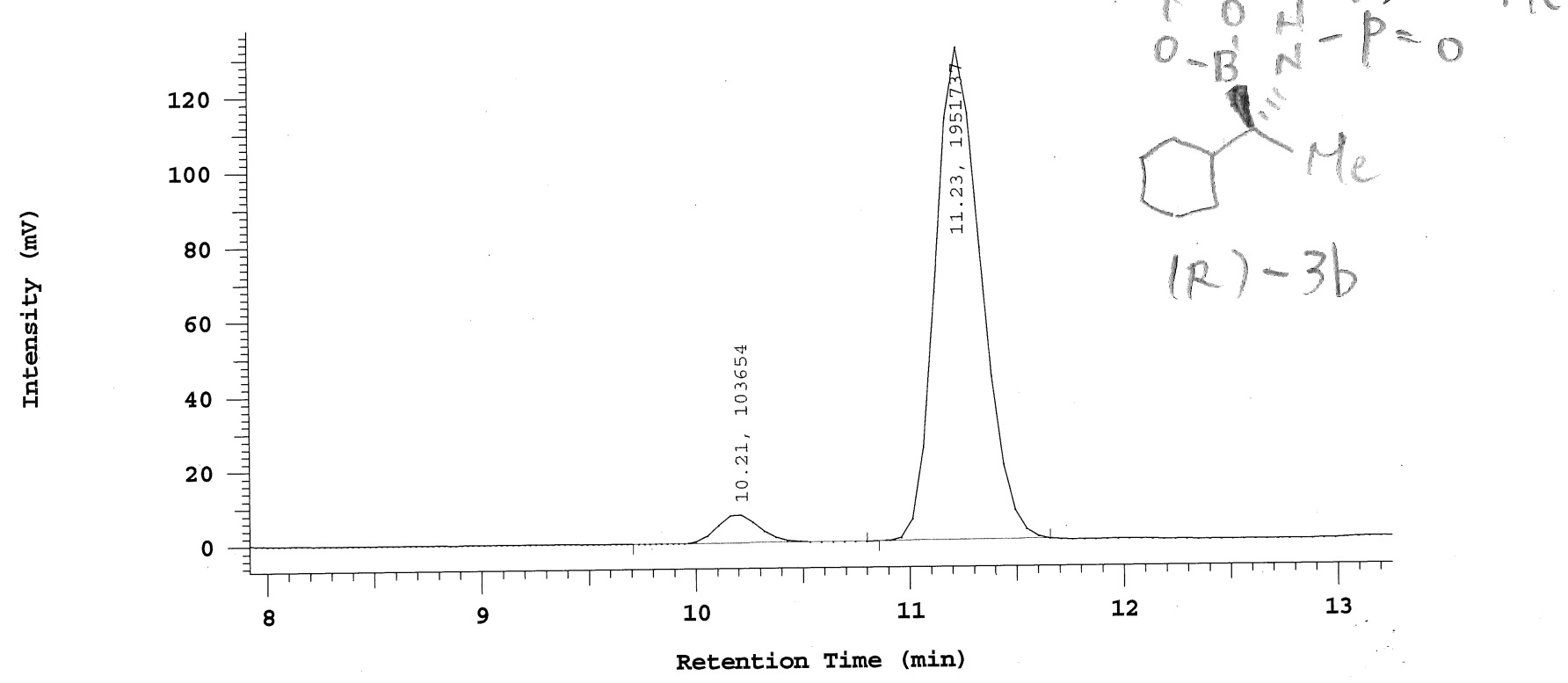

Processing Method: 10/90 iPrOH/Hexane

Column Type: OD-H 2

Method Developer: Administrator

Pump A: L-2130

Pump A Solvent A: Hexane

Pump A Solvent C: iPrOH

Pump A Solvent B: 10/90 iPrOH/Hexane Pump A Solvent D: iPrOH

Method Description:

Chrom Type: HPLC Channel : 1

Peak Quantitation: AREA

Calculation Method: AREA\%

\begin{tabular}{rrrr} 
No. & RT & Area & Area \% \\
\cline { 2 - 4 } 1 & 10.21 & 103654 & 5.043 \\
2 & 11.23 & 1951737 & 94.957 \\
\hline & & 2055391 & 100.000
\end{tabular}

Peak rejection level: 0 


\section{D-2000 Elite HPLC System Manager Report}

Analyzed Date and Time: 2020/04/15

$$
13: 41
$$

Reported Date and Time: 2020/04/15

Processed Date and Time: 2020/04/15

$$
15: 23
$$

Data Path: C: \WIN32APP \D2000HSM \Isocratic \DATA $\backslash 2969 \backslash$

Processing Method: 10/90 iPrOH/Hexane

\section{System (acquisition): Sys 1}

Application (data) : Isocratic HPLC

Sample Name: MUR-211-rac-OZ3-10\%

Injection from this vial: 1 of 1

Sample Description:
Series: 2969

Vial Number: 144

Vial Type: UNK

Volume: $10.0 \mathrm{ul}$

Chrom Type: HPLC Channel : 1

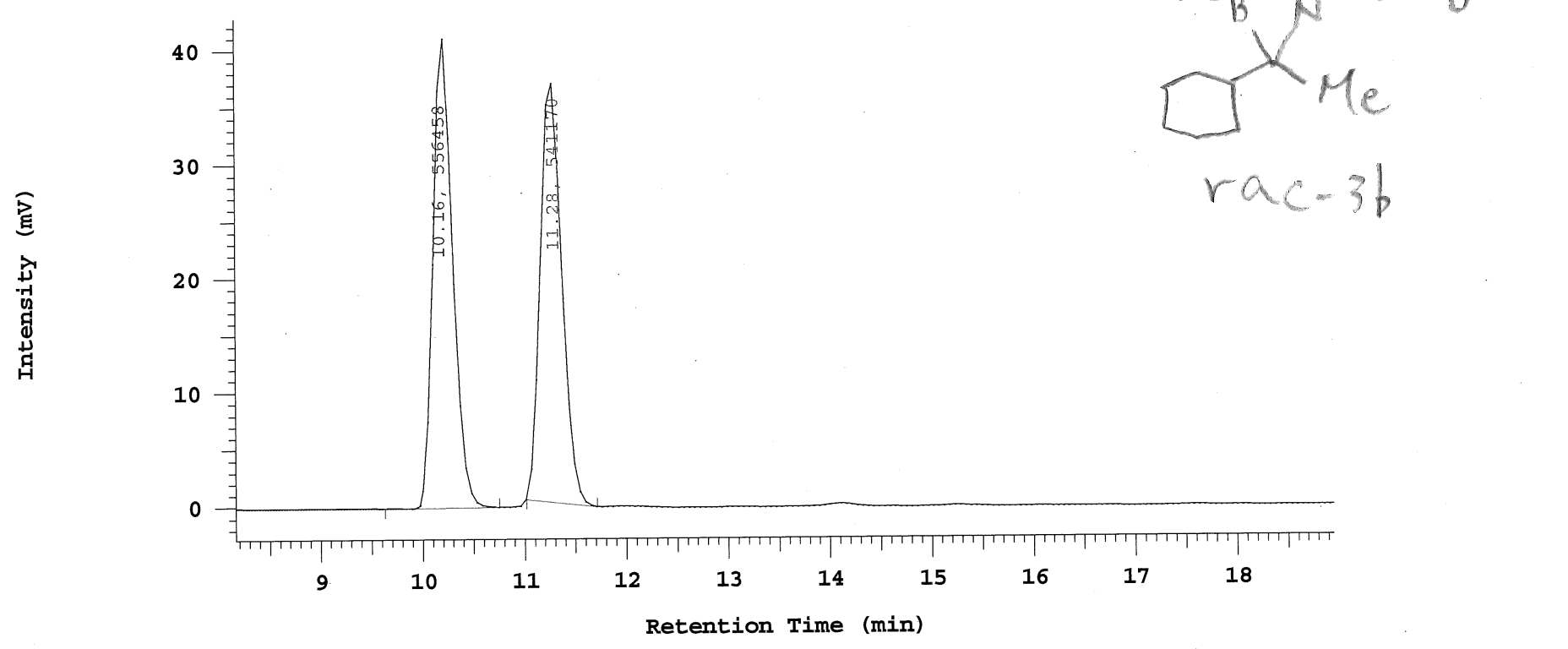

Processing Method: 10/90 iProH/Hexane

Column Type: OD-H 2

Method Developer: Administrator

Pump A: L-2130

Pump A Solvent A: Hexane

Pump A Solvent B: 10/90 iProH/Hexane

Pump A Solvent C: iPrOH

Pump A solvent D: iPrOH

Method Description:

Chrom Type: HPLC Channel : 1

Peak Quantitation: AREA

Calculation Method: AREA\%

\begin{tabular}{rrrr} 
No. & RT & Area & Area \% \\
\hline 1 & 10.16 & 556458 & 50.696 \\
2 & 11.28 & 541170 & 49.304 \\
\hline & & 1097628 & 100.000
\end{tabular}

Peak rejection level: 0 


\section{D-2000 Elite HPLC System Manager Report}

Analyzed Date and Time: 2020/11/22

Reported Date and Time: 2020/11/22

$$
14: 47
$$

Processed Date and Time: 2020/11/22

$$
16: 54
$$

Data Path: C: \WIN32APP \D2000HSM \Isocratic \DATA \3104\

Processing Method: 10/90 iPrOH/Hexane

System (acquisition): Sys 1

Application(data): Isocratic HPLC

Sample Name: MUR-145-OZ3-10\%

Injection from this vial: 1 of 1

Sample Description:
Series: 3104

Vial Number: 149

Vial Type: UNK

Volume: $10.0 \mathrm{ul}$

Chrom Type: HPLC Channel : 1

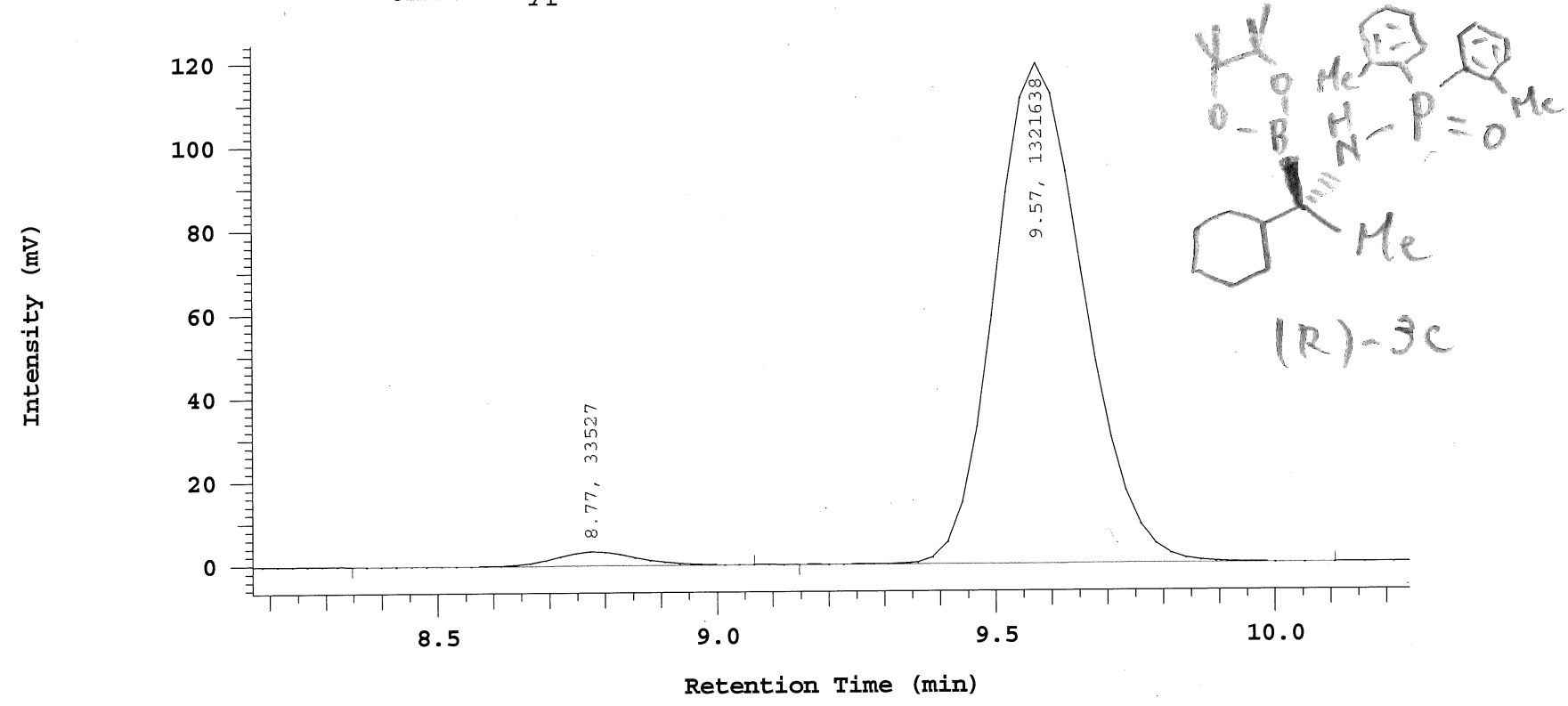

Processing Method: 10/90 iPrOH/Hexane

Column Type: OD-H 2

Method Developer: Administrator

Pump A: L-2130

Pump A Solvent A: Hexane

Pump A Solvent B: 10/90 iProH/Hexane

Pump A Solvent C: iPrOH

Pump A Solvent D: iPrOH

Method Description:

Chrom Type: HPLC Channel : 1

Peak Quantitation: AREA

Calculation Method: AREA\%

\begin{tabular}{rrrr} 
No. & RT & Area & Area \% \\
\hline 1 & 8.77 & 33527 & 2.474 \\
2 & 9.57 & 1321638 & 97.526 \\
\hline & & 1355165 & 100.000
\end{tabular}

Peak rejection level: 0 


\section{D-2000 Elite HPLC System Manager Report}

Analyzed Date and Time: 2020/11/22 $18: 28$

Reported Date and Time: 2020/11/24

Processed Date and Time: $2020 / 11 / 24$

$09: 28$

Data Path: C: $\backslash$ WIN32APP $\backslash$ D2000HSM $\backslash$ Isocratic $\backslash$ DATA $\backslash 3105 \backslash$

Processing Method: 10/90 iProH/Hexane

System (acquisition): Sys 1

Series: 3105

Application(data): Isocratic HPLC

Sample Name: MUR-1c-rac

Injection from this vial: 1 of 1

Vial Number: 150

Vial Type: UNK

Sample Description:

Volume: $10.0 \mathrm{ul}$

Chrom Type: HPLC Channel : 1

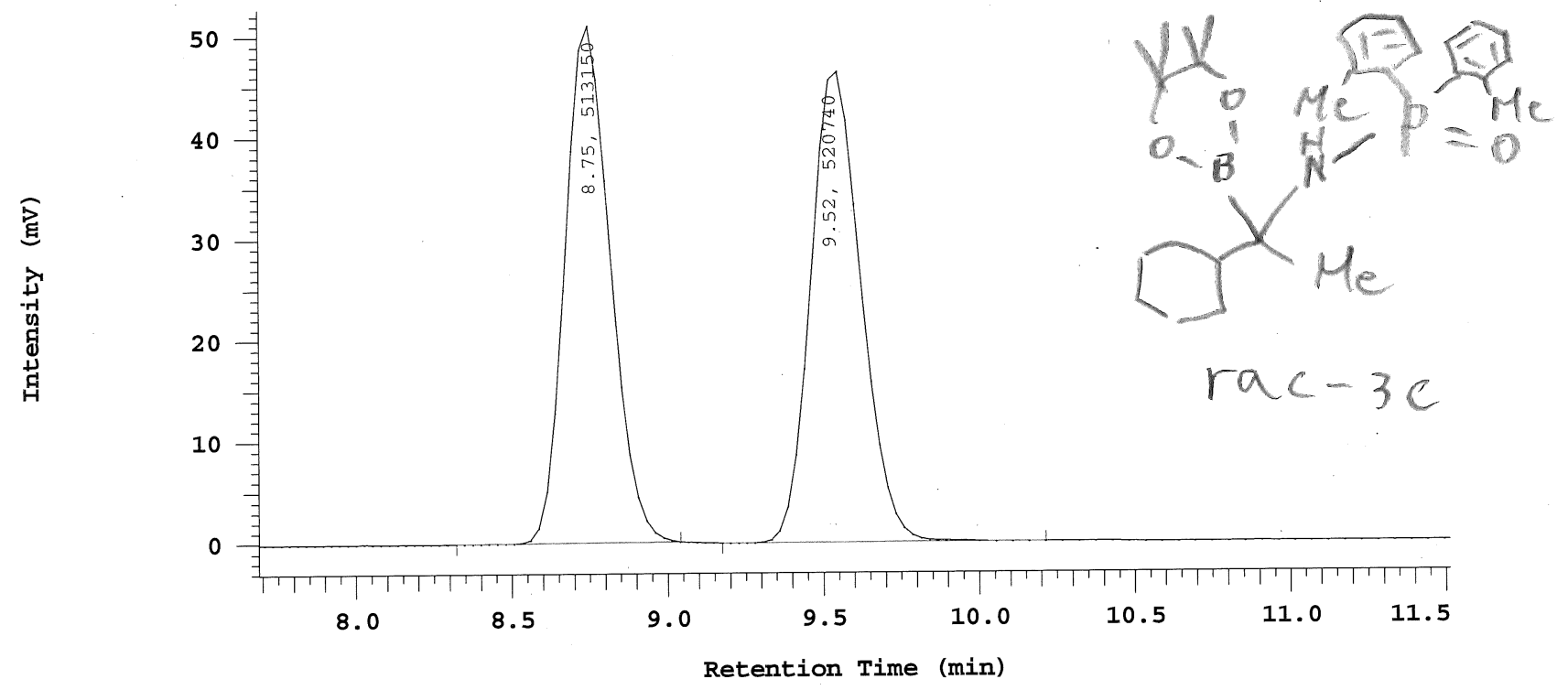

Processing Method: 10/90 iProH/Hexane

Column Type: OD-H 2

Method Developer: Administrator

Pump A: L-2130

Pump A Solvent A: Hexane

Pump A Solvent B: 10/90 iProH/Hexane

Pump A Solvent C: iPrOH

Pump A Solvent D: iPrOH

Method Description:

Chrom Type: HPLC Channel : 1

Peak Quantitation: AREA

Calculation Method: AREA\%

\begin{tabular}{rrrr} 
No. & RT & Area & Area \% \\
\hline 1 & 8.75 & 513150 & 49.633 \\
2 & 9.52 & 520740 & 50.367 \\
\hline & & 1033890 & 100.000 \\
\hline
\end{tabular}

Peak rejection level: 0 


\section{D-2000 Elite HPLC System Manager Report}

Analyzed Date and Time: 2019/12/21

$$
12: 00
$$

Reported Date and Time: 2019/12/21

Processed Date and Time: 2019/12/21

$13: 35$

Data Path: C: \WIN32APP \D2000HSM Isocratic \DATA \2939

Processing Method: 10/90 iPrOH/Hexane

System (acquisition): Sys 1

Application(data): Isocratic HPLC

Sample Name: MUR-146-OZ3-10\%

Series: 2939

Injection from this vial: 1 of 1

Sample Description:

Vial Number: 143

Vial Type: UNK

Volume: $10.0 \mathrm{ul}$

Chrom Type: HPLC Channel : 1

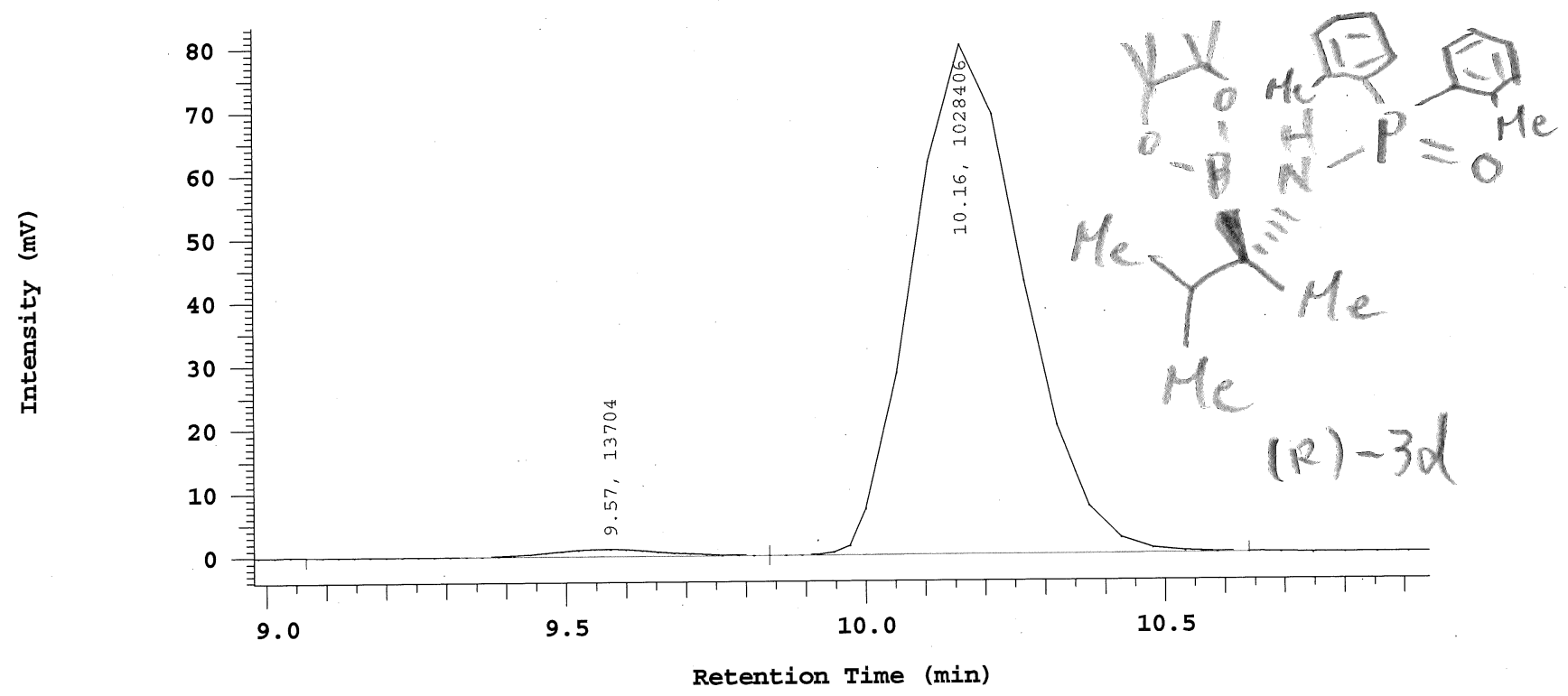

Processing Method: 10/90 iPrOH/Hexane

Column Type: OD-H 2

Method Developer: Administrator

Pump A: L-2130

Pump A Solvent A: Hexane

Pump A Solvent B: 10/90 iProH/Hexane

Pump A solvent C: iPrOH

Pump A Solvent D: iPrOH

Method Description:

Chrom Type: HPLC Channel : 1

Peak Quantitation: AREA

Calculation Method: AREA\%

\begin{tabular}{rrrr}
\multicolumn{1}{c}{ RT } & \multicolumn{1}{c}{ Area } & Area \% \\
\cline { 2 - 4 } 1 & 9.57 & 13704 & 1.315 \\
2 & 10.16 & 1028406 & 98.685 \\
\hline & & 1042110 & 100.000
\end{tabular}

Peak rejection level: 0 


\section{D-2000 Elite HPLC System Manager Report}

Analyzed Date and Time: 2019/12/21 $13: 42$

Reported Date and Time: 2020/01/23

Processed Date and Time: 2020/01/23

$$
19: 58
$$

Data Path: C: \WIN32APP \D2000HSM \Isocratic \DATA $\backslash 2940 \backslash$

Processing Method: 10/90 iPrOH/Hexane

System (acquisition): Sys 1

Application(data): Isocratic HPLC

Sample Name: MUR-rac 147

Injection from this vial: 1 of 1

Sample Description:
Series: 2940

Vial Number: 144

Vial Type: UNK

Volume: $10.0 \mathrm{ul}$

Chrom Type: HPLC Channel : 1

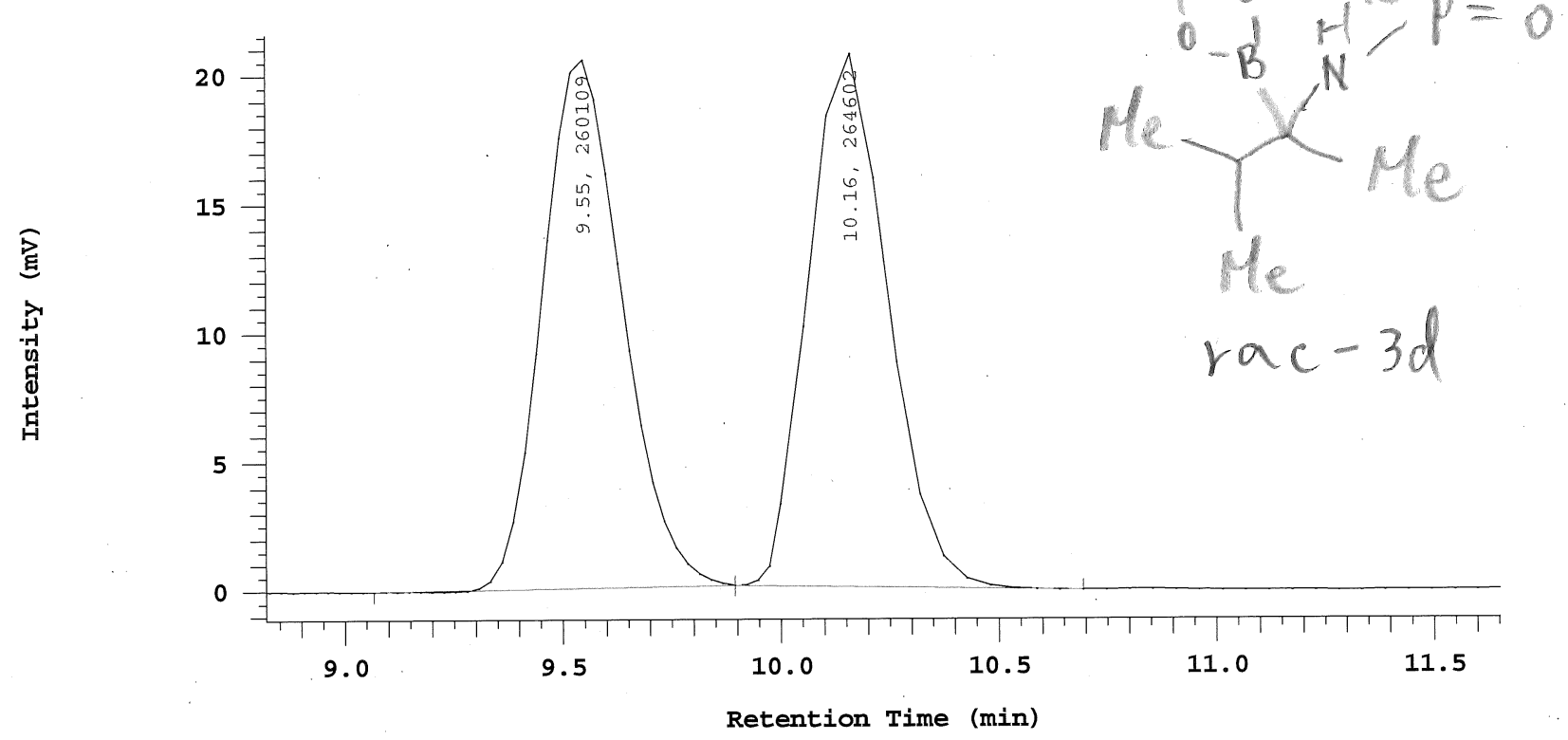

Processing Method: 10/90 iProH/Hexane

Column Type: OD-H 2

Method Developer: Administrator

Pump A: L-2130

Pump A Solvent A: Hexane

Pump A Solvent B: 10/90 iProH/Hexane

Pump A Solvent C: iPrOH

Pump A Solvent D: iPrOH

Method Description:

Chrom Type: HPLC Channel : 1

Peak Quantitation: AREA

Calculation Method: AREA\%

\begin{tabular}{rrrr} 
No. & \multicolumn{1}{c}{ RT } & Area & Area \% \\
\hline 1 & 9.55 & 260109 & 49.572 \\
2 & 10.16 & 264602 & 50.428 \\
\hline & & 524711 & 100.000
\end{tabular}

Peak rejection level: 0 


\section{Chromaster System Manager Report}

Analyzed Date and Time: 2019/12/24

Reported Date and Time: 2019/12/24

$00: 17$

$09: 55: 33$

Processed Date and Time: 2019/12/24

$09: 55$

Data Path: C: \WIN32APP \CHROMASTER \MUR \DATA \0002 \

Processing Method: column1(IA-3)

System (acquisition): Sys 1

Series: 0002

Application (data): MUR

Sample Name: MUR-148-IA-8\%

Injection from this vial: 1 of 1

Vial Number: 10

Vial Type: UNK

Sample Description:

Volume: 10.0 ul

Chrom Type: Fixed WL Chromatogram, $250 \mathrm{~nm}$

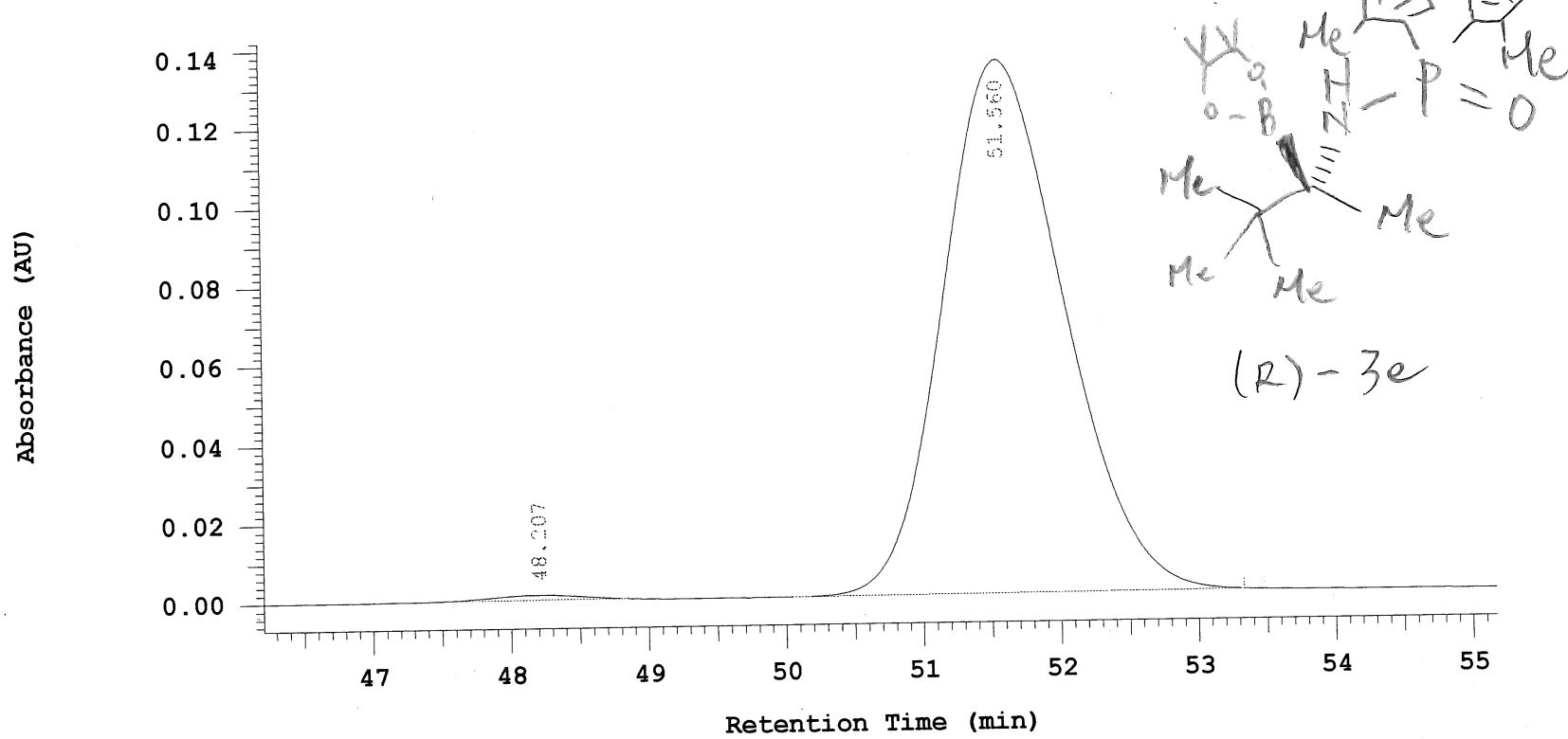

Processing Method: column1(IA-3)

Method Developer:

Pump 1: 5110
Pump 1 Solvent A: hexane
Pump 1 Solvent B: 2-propanol
Pump 1 Solvent $C$ :
Pump 1 Solvent D:

Method Description:

Chrom Type: Fixed WL Chromatogram, $250 \mathrm{~nm}$

Peak Quantitation: AREA

Calculation Method: AREA\%

\begin{tabular}{rrrrr} 
No. & RT & Area & Conc 1 & BC \\
\cline { 5 - 6 } 1 & 48.207 & 25084 & 0.607 & BB \\
2 & 51.560 & 4108822 & 99.393 & BB \\
\hline
\end{tabular}

Peak rejection level: 0 


\section{Chromaster System Manager Report}

Analyzed Date and Time: 2019/12/23 $22: 05$

Reported Date and Time: 2019/12/24

Processed Date and Time: 2019/12/24

$$
09: 53
$$

Data Path: C: \WIN32APP \CHROMASTER \MUR \DATA \0001

Processing Method: column1(IA-3)

System (acquisition): Sys 1

Series: 0001

Application (data): MUR

Sample Name: MUR-149-IA-8\%

Injection from this vial: 1 of 1

Vial Number: 9

Vial Type: UNK

Sample Description:

Volume: $10.0 \mathrm{ul}$

Chrom Type: Fixed WL Chromatogram, $250 \mathrm{~nm}$

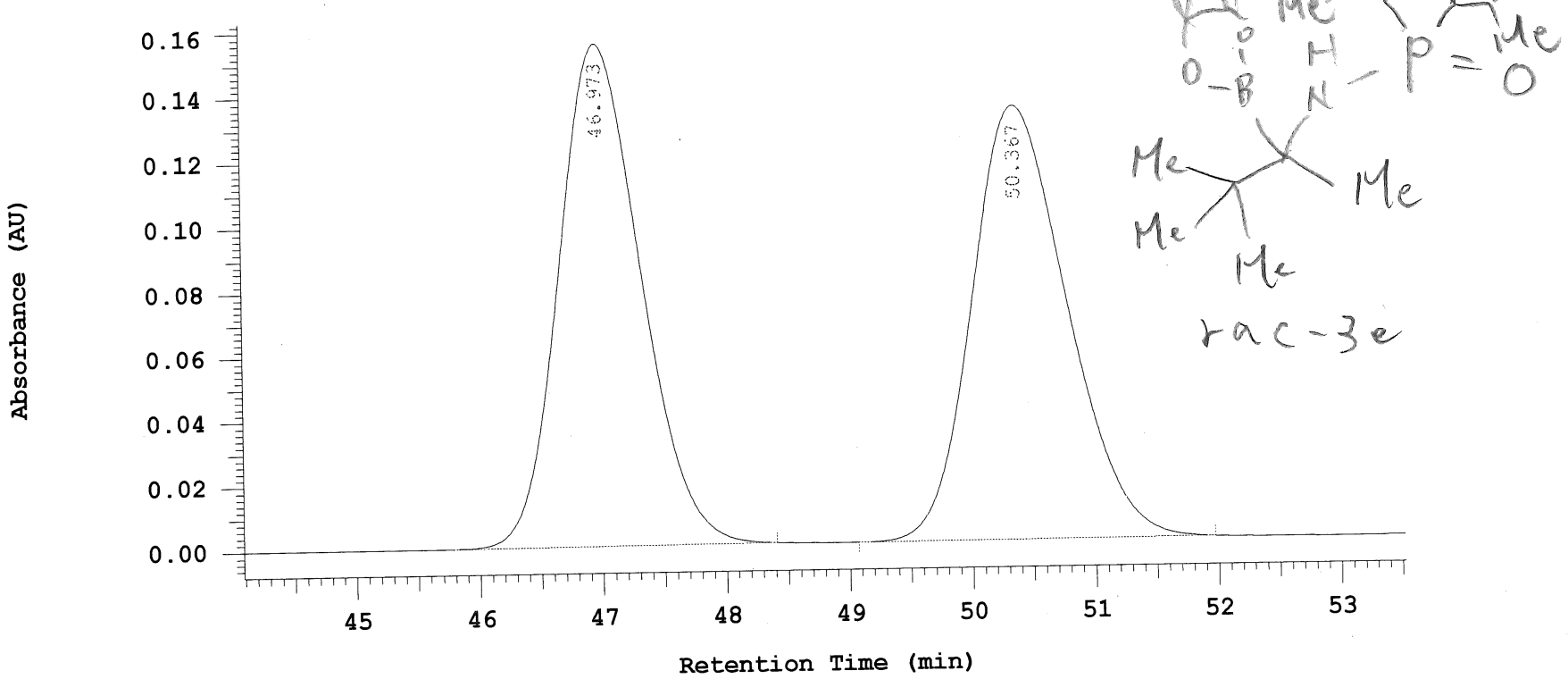

Processing Method: column1(IA-3)

Method Developer:

Pump 1: 5110
Pump 1 Solvent A: hexane
Pump 1 Solvent B: 2-propanol
Pump 1 Solvent $C$ :
Pump 1 Solvent D:

Method Description:

Chrom Type: Fixed WL Chromatogram, $250 \mathrm{~nm}$

Peak Quantitation: AREA

Calculation Method: AREA\%

\begin{tabular}{|c|c|c|c|c|}
\hline No. & RT & Area & Conc 1 & $\mathrm{BC}$ \\
\hline $\begin{array}{l}1 \\
2\end{array}$ & $\begin{array}{l}46.973 \\
50.367\end{array}$ & $\begin{array}{l}3579983 \\
3588904\end{array}$ & $\begin{array}{l}49.938 \\
50.062\end{array}$ & $\begin{array}{l}\mathrm{BB} \\
\mathrm{BB}\end{array}$ \\
\hline & & 7168887 & 100.000 & \\
\hline
\end{tabular}

Peak rejection level: 0 


\section{D-2000 Elite HPLC System Manager Report}

Analyzed Date and Time: 2020/06/24

Reported Date and Time: 2020/06/24

$14: 22$

$15: 43$

Processed Date and Time: 2020/06/24

$$
15: 43
$$

Data Path: C: \WIN32APP \D2000HSM \Isocratic \DATA \3017\

Processing Method: 10/90 iProH/Hexane

System (acquisition): Sys 1

Application(data): Isocratic HPLC

Sample Name: MUR-249-OZ3-10\%

Series: 3017

Injection from this vial: 1 of 1

Vial Number: 142

Vial Type: UNK

Sample Description:

Volume: $10.0 \mathrm{ul}$

Chrom Type: HPLC Channel : 1

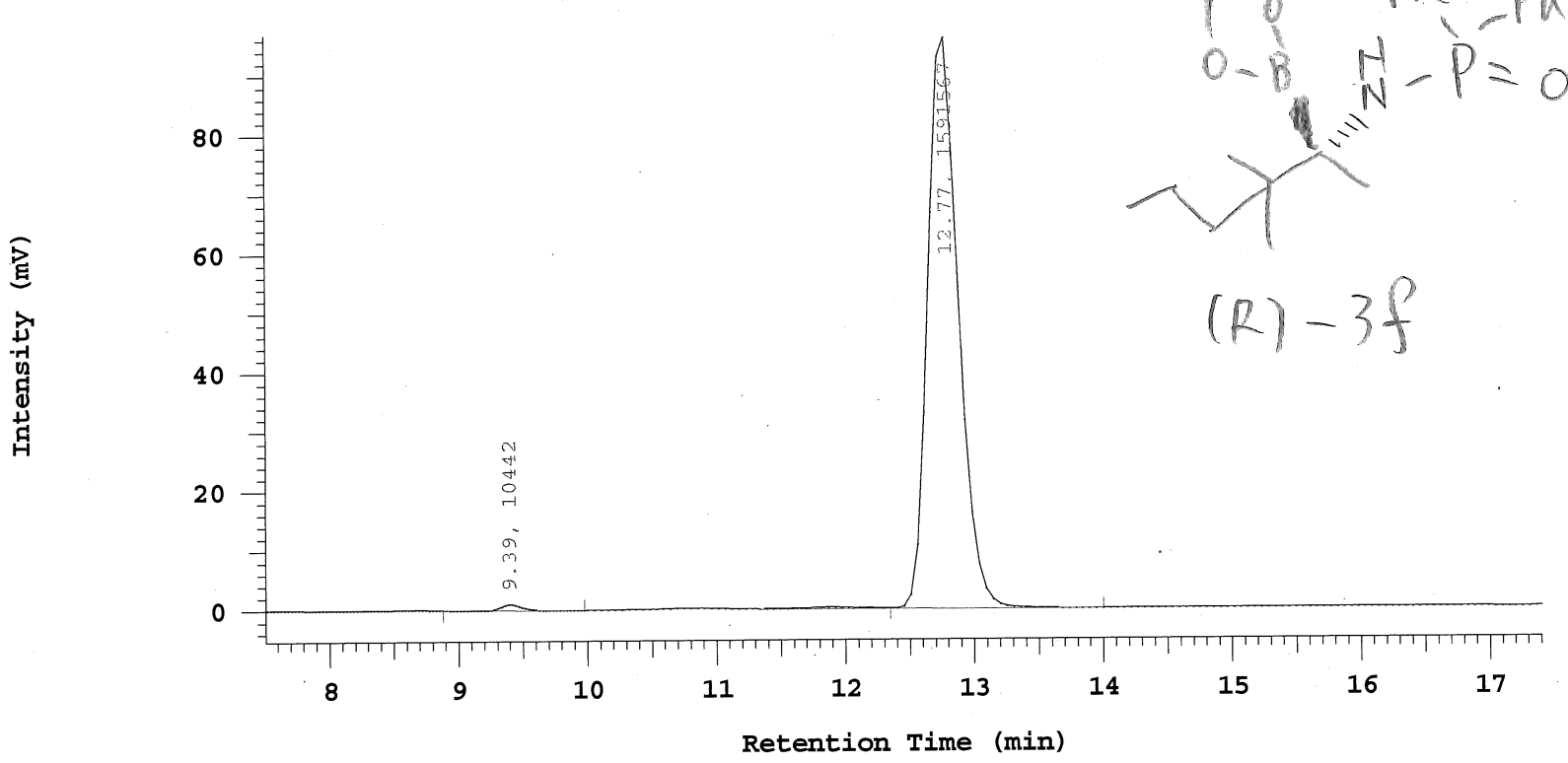

Processing Method: 10/90 iProH/Hexane

Column Type: OD-H 2

Method Developer: Administrator

Pump A: L-2130

Pump A Solvent A: Hexane

Pump A Solvent B: 10/90 iProH/Hexane

Pump A Solvent C: iPrOH

Pump A Solvent D: iProH

Method Description:

Chrom Type: HPLC Channel : 1

Peak Quantitation: AREA

Calculation Method: AREA\%

\begin{tabular}{rrrr} 
No. & \multicolumn{1}{c}{ RT } & Area & Area $\%$ \\
\hline 1 & 9.39 & 10442 & 0.652 \\
2 & 12.77 & 1591567 & 99.348 \\
\hline & & 1602009 & 100.000
\end{tabular}

Peak rejection level: 0 
D-2000: Isocrati Series: 3018

Report Name: modified System: Sys 1 C HPLC

\section{D-2000 Elite HPLC System Manager Report}

Analyzed Date and Time: 2020/06/24 $15: 29$

Reported Date and Time: 2020/06/24 $17: 13$

Processed Date and Time: 2020/06/24

$$
17: 12
$$

Data Path: C: \WIN32APP \D2000HSM Isocratic \DATA \3018\ Processing Method: 10/90 iPrOH/Hexane System (acquisition): Sys 1 Application(data) : Isocratic HPLC Sample Name: MUR-247-rac-OZ3-10\% Injection from this vial: 1 of 1 Sample Description:

Chrom Type: HPLC Channel : 1

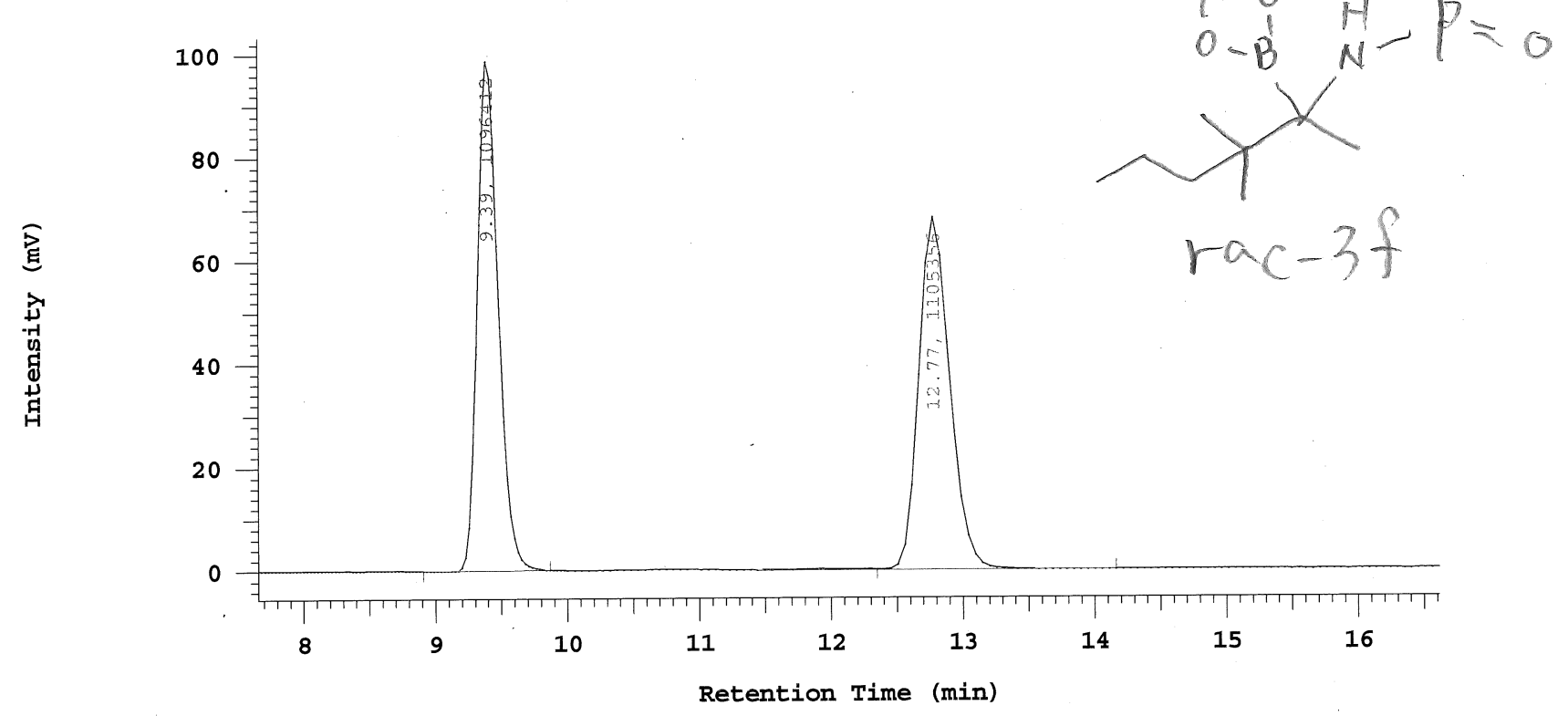

Series: 3018

Vial Number: 143

Vial Type: UNK

Volume: $10.0 \mathrm{ul}$

Processing Method: 10/90 iProH/Hexane Column Type: OD-H 2 Method Developer: Administrator Pump A: L-2130

Pump A Solvent A: Hexane Pump A Solvent B: 10/90 iProH/Hexane

Pump A Solvent C: iProH Pump A solvent D: iPrOH Method Description:

Chrom Type: HPLC Channel : 1

Peak Quantitation: AREA

Calculation Method: AREA\%

\begin{tabular}{rrrr} 
No. & \multicolumn{1}{c}{ RT } & Area & Area \% \\
\hline 1 & 9.39 & 1096412 & 49.797 \\
2 & 12.77 & 1105356 & 50.203 \\
\hline & & 2201768 & 100.000
\end{tabular}

Peak rejection level: 0 


\section{D-2000 Elite HPLC System Manager Report}

Analyzed Date and Time: 2020/07/04

$$
17: 44
$$

Reported Date and Time: 2020/09/02

Processed Date and Time: 2020/09/02

$$
20: 47
$$

Data Path: C: $\backslash$ WIN32APP \D2000HSM \Isocratic $\backslash$ DATA $\backslash 3030 \backslash$

Processing Method: 10/90 iProH/Hexane

System (acquisition): Sys 1

Series: 3030

Application(data): Isocratic HPLC

Sample Name: MUR-265-0Z3-10\%

Vial Number: 142

Injection from this vial: 1 of 1

Vial Type: UNK

Sample Description:

Volume: $10.0 \mathrm{ul}$

Chrom Type: HPLC Channel : 1

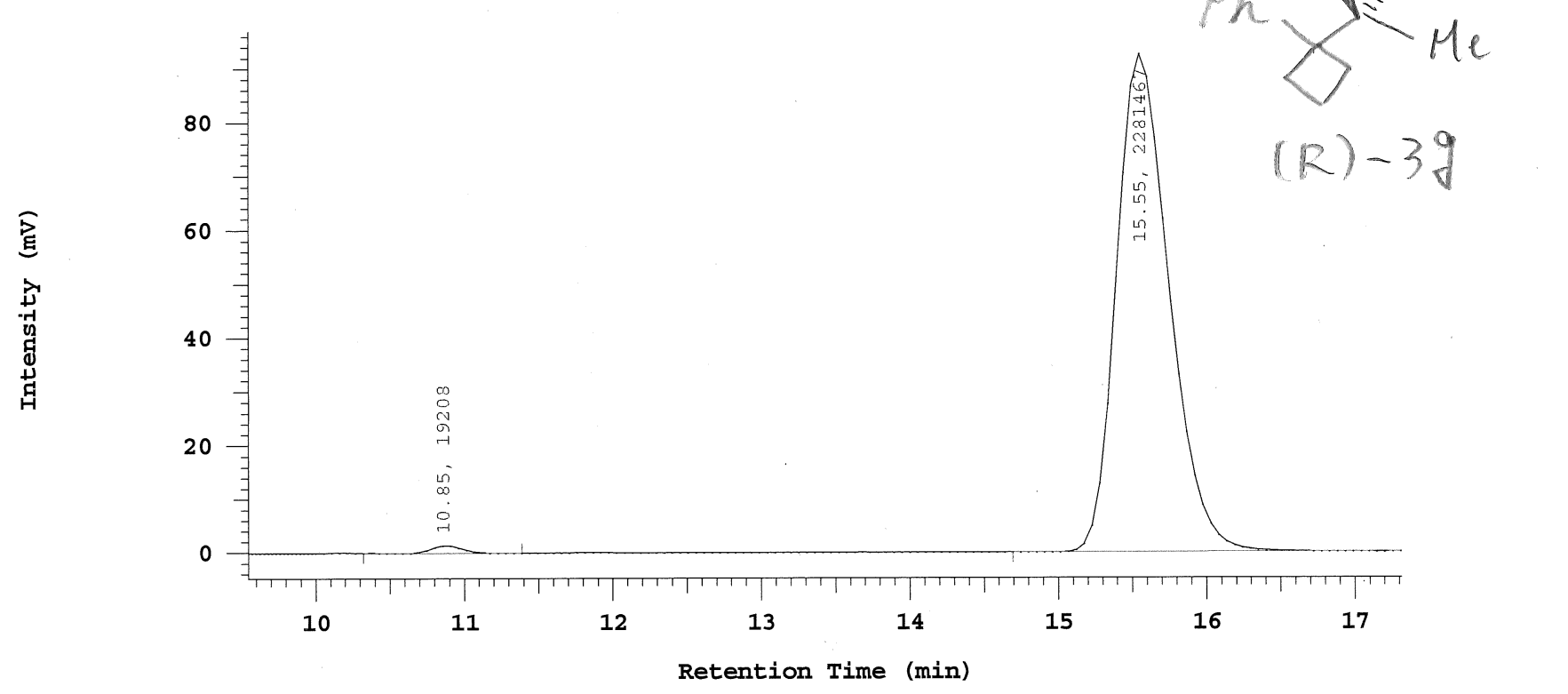

Processing Method: 10/90 iProH/Hexane

Column Type: OD-H 2

Method Developer: Administrator

Pump A: L-2130

Pump A Solvent A: Hexane

Pump A Solvent B: 10/90 iProH/Hexane

Pump A solvent C: iProH

Pump A Solvent D: iPrOH

Method Description:

Chrom Type: HPLC Channel : 1

Peak Quantitation: AREA

Calculation Method: AREA\%

\begin{tabular}{rrrr} 
No. & RT & Area & Area $\%$ \\
\hline 1 & 10.85 & 19208 & 0.835 \\
2 & 15.55 & 2281467 & 99.165 \\
\hline & & 2300675 & 100.000
\end{tabular}

Peak rejection level: 0 


\section{D-2000 Elite HPLC System Manager Report}

Analyzed Date and Time: 2020/07/04

$$
16: 52
$$

Reported Date and Time: 2020/09/02

Processed Date and Time: 2020/09/02

$$
20: 47
$$

Data Path: C: \WIN32APP \D2000HSM \Isocratic \DATA \3029 Processing Method: 10/90 iPrOH/Hexane

System (acquisition): Sys 1

Application(data): Isocratic HPLC

Sample Name: MUR-262-rac-0Z3-10\%

Injection from this vial: 1 of. 1

Sample Description:
Series: 3029

Vial Number: 141

Vial Type: UNK

Volume: $10.0 \mathrm{ul}$

Chrom Type: HPLC Channel : 1

家

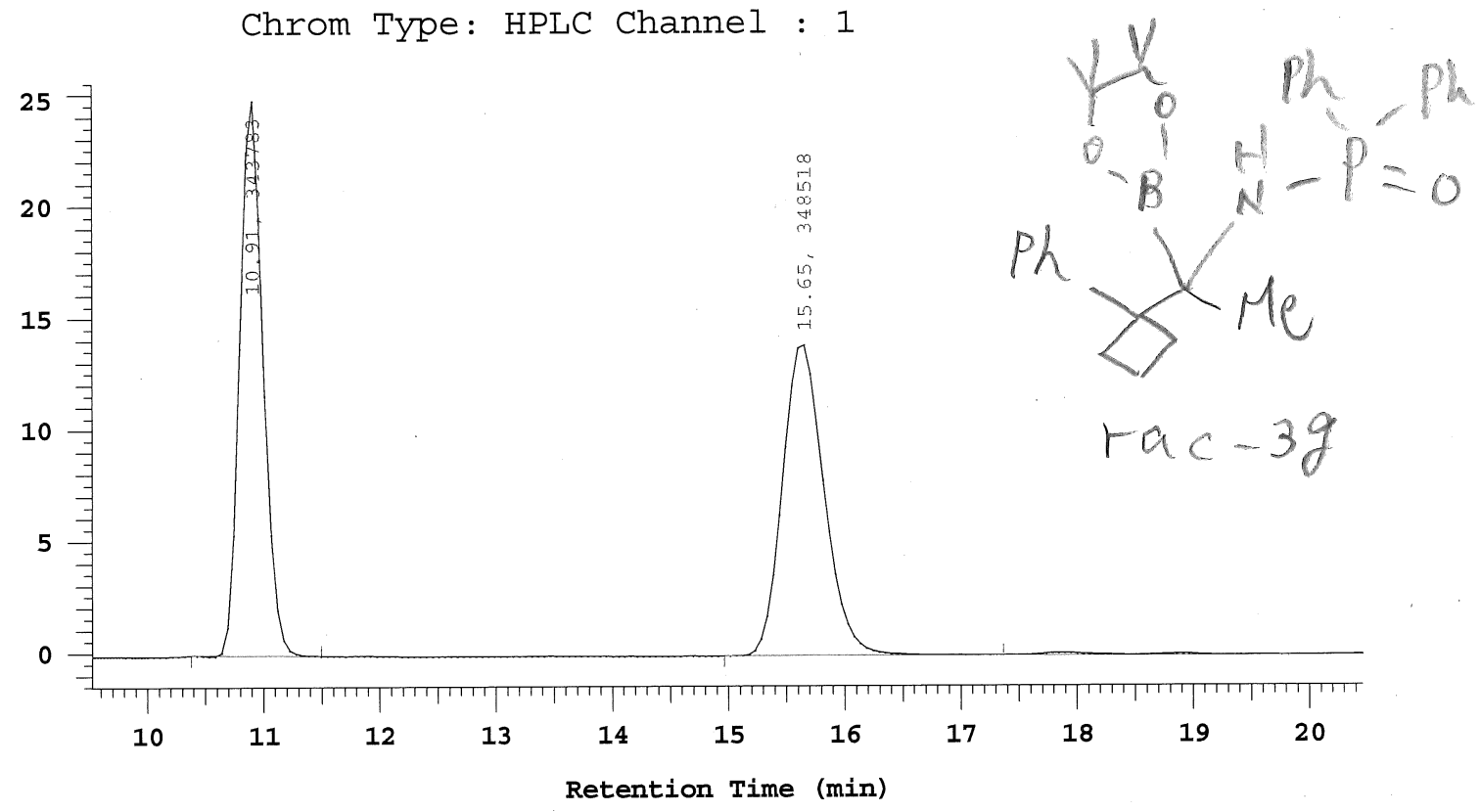

Processing Method: 10/90 iProH/Hexane

Column Type: OD-H 2

Method Developer: Administrator

Pump A: L-2130

Pump A Solvent A: Hexane

Pump A Solvent B: 10/90 iProH/Hexane

Pump A Solvent C: iPrOH

Pump A Solvent D: iPrOH

Method Description:

Chrom Type: HPLC Channel : 1

Peak Quantitation: AREA

Calculation Method: AREA\%

\begin{tabular}{rccr} 
No. & RT & Area & Area \% \\
\hline 1 & 10.91 & 343783 & 49.658 \\
2 & 15.65 & 348518 & 50.342 \\
\hline & & 692301 & 100.000
\end{tabular}

Peak rejection level: 0 


\section{D-2000 Elite HPLC System Manager Report}

Analyzed Date and Time: 2020/08/03

$$
16: 39
$$

Reported Date and Time: 2020/08/03

Processed Date and Time: 2020/08/03

$$
18: 21
$$

Data Path: C: \WIN32APP \D2000HSM Isocratic \DATA \3040

Processing Method: 10/90 iPrOH/Hexane

System (acquisition): Sys 1

Series: 3040

Application(data): Isocratic HPLC

Vial Number: 142

Sample Name: MUR-279-L22-OZ3-10\%

Vial Type: UNK

Injection from this vial: 1 of 1

Volume: $10.0 \mathrm{ul}$

Sample Description:

Chrom Type: HPLC Channel : 1

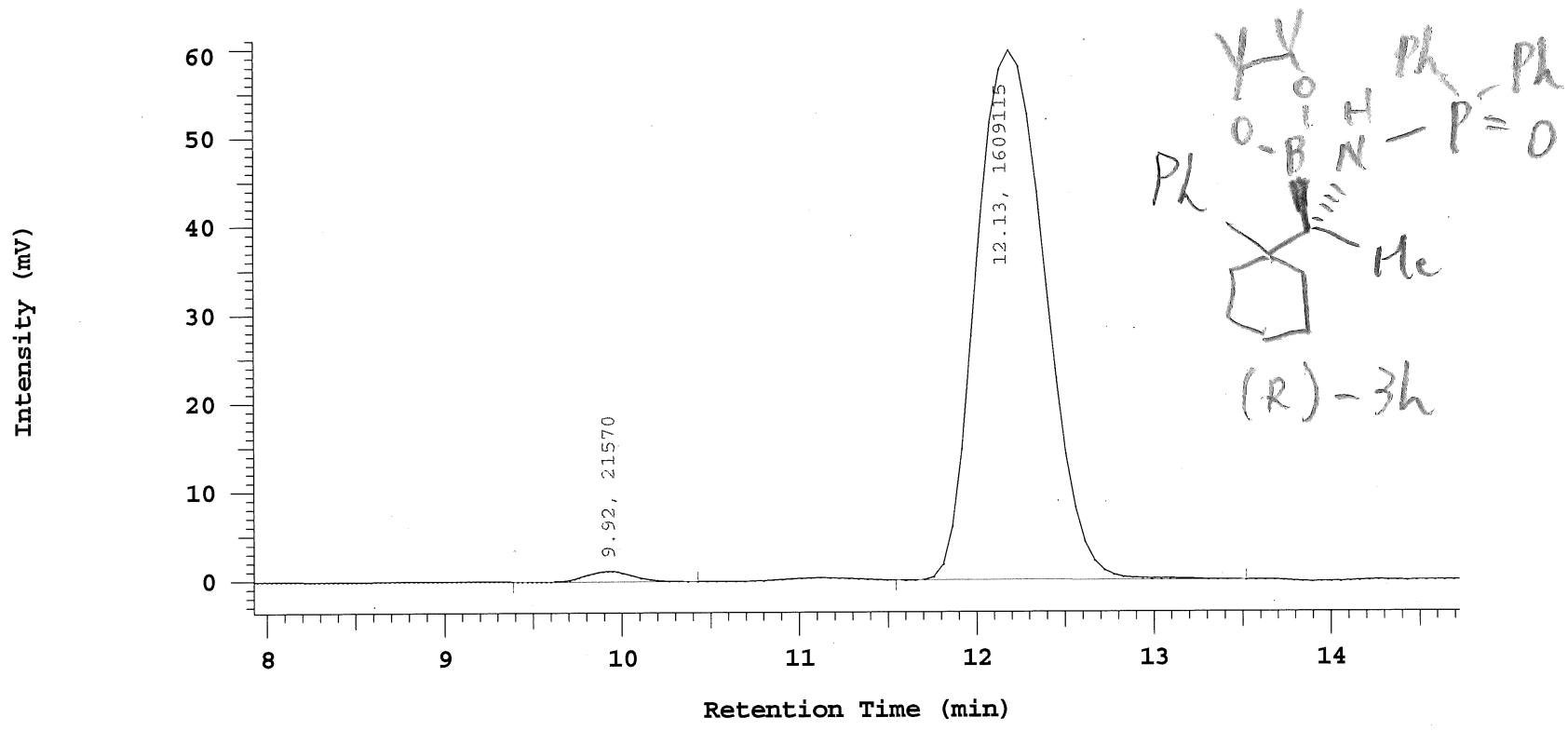

Processing Method: 10/90 iPrOH/Hexane

Column Type: OD-H 2

Method Developer: Administrator

Pump A: L-2130

Pump A Solvent A: Hexane

Pump A Solvent B: 10/90 iPrOH/Hexane

Pump A Solvent C: iPrOH

Pump A Solvent D: iPrOH

Method Description:

Chrom Type: HPLC Channel : 1

Peak Quantitation: AREA

Calculation Method: AREA\%

\begin{tabular}{rrrr} 
No. & \multicolumn{1}{c}{ RT } & Area & Area $\%$ \\
\hline 1 & 9.92 & 21570 & 1.323 \\
2 & 12.13 & 1609115 & 98.677 \\
\hline & & 1630685 & 100.000
\end{tabular}

Peak rejection level: 0 


\section{D-2000 Elite HPLC System Manager Report}

Analyzed Date and Time: 2020/08/03

$$
15: 27
$$

Reported Date and Time: 2020/08/03

$16: 48$

Processed Date and Time: 2020/08/03

$$
16: 48
$$

Data Path: C: \WIN32APP \D2000HSM \Isocratic \DATA \3039

Processing Method: 10/90 iPrOH/Hexane

System (acquisition): Sys 1

Application(data): Isocratic HPLC

Sample Name: MUR-280-rac-OZ3-10\%

Injection from this vial: 1 of 1

Sample Description:
Series: 3039

Vial Number: 141

Vial Type: UNK

Volume: $10.0 \mathrm{ul}$

Chrom Type: HPLC Channel : 1

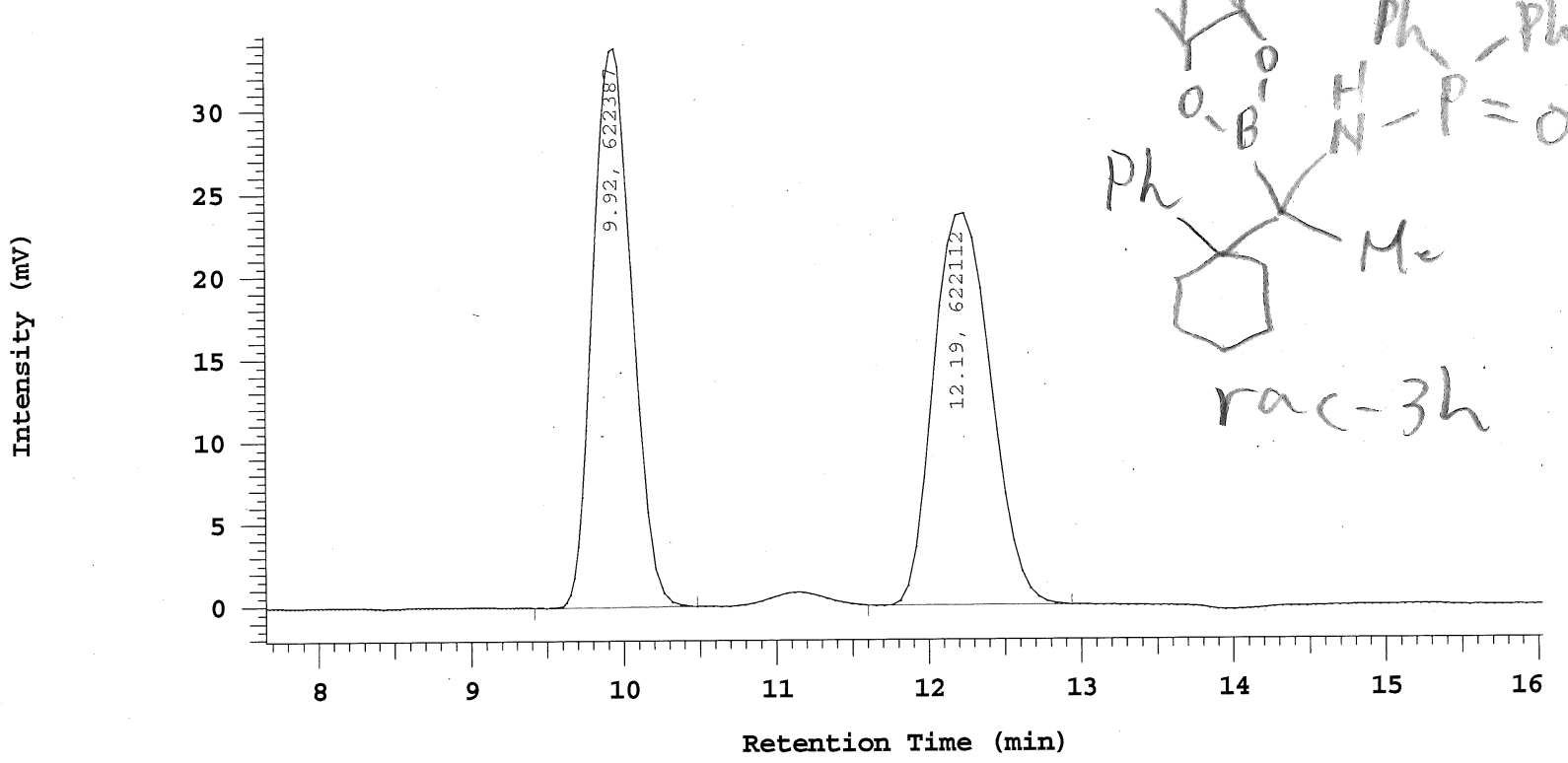

Processing Method: 10/90 iProH/Hexane

Column Type: OD-H 2

Method Developer: Administrator

Pump A: L-2130

Pump A Solvent A: Hexane

Pump A Solvent B: 10/90 iProH/Hexane

Pump A solvent C: iProH

Pump A Solvent D: iPrOH

Method Description:

Chrom Type: HPLC Channel : 1

Peak Quantitation: AREA

Calculation Method: AREA\%

\begin{tabular}{rrrr} 
No. & \multicolumn{1}{c}{ RT } & Area & Area $\%$ \\
\hline 1 & 9.92 & 622387 & 50.011 \\
2 & 12.19 & 622112 & 49.989 \\
\hline & & 1244499 & 100.000
\end{tabular}

Peak rejection level: 0 
D-2000: Isocrati Series: 3025

Report Name: modified System: Sys 1 C HPLC

\section{D-2000 Elite HPLC System Manager Report}

$\begin{array}{rl}\text { Analyzed Date and Time: } & 2020 / 06 / 29 \quad \text { Reported Date and Time: 2020/06/29 } \\ 15: 44 & 17: 39\end{array}$

Processed Date and Time: 2020/06/29

$17: 39$

Data Path: C: \WIN32APP \D2000HSM \Isocratic \DATA \3025

Processing Method: 10/90 iPrOH/Hexane

System (acquisition): Sys 1

Series: 3025

Application(data): Isocratic HPLC

Sample Name: MUR-260-OZ3-10\%

Vial Number: 141

Injection from this vial: 1 of 1

Vial Type: UNK

Sample Description:

Volume: $10.0 \mathrm{ul}$

Chrom Type: HPLC Channel : 1

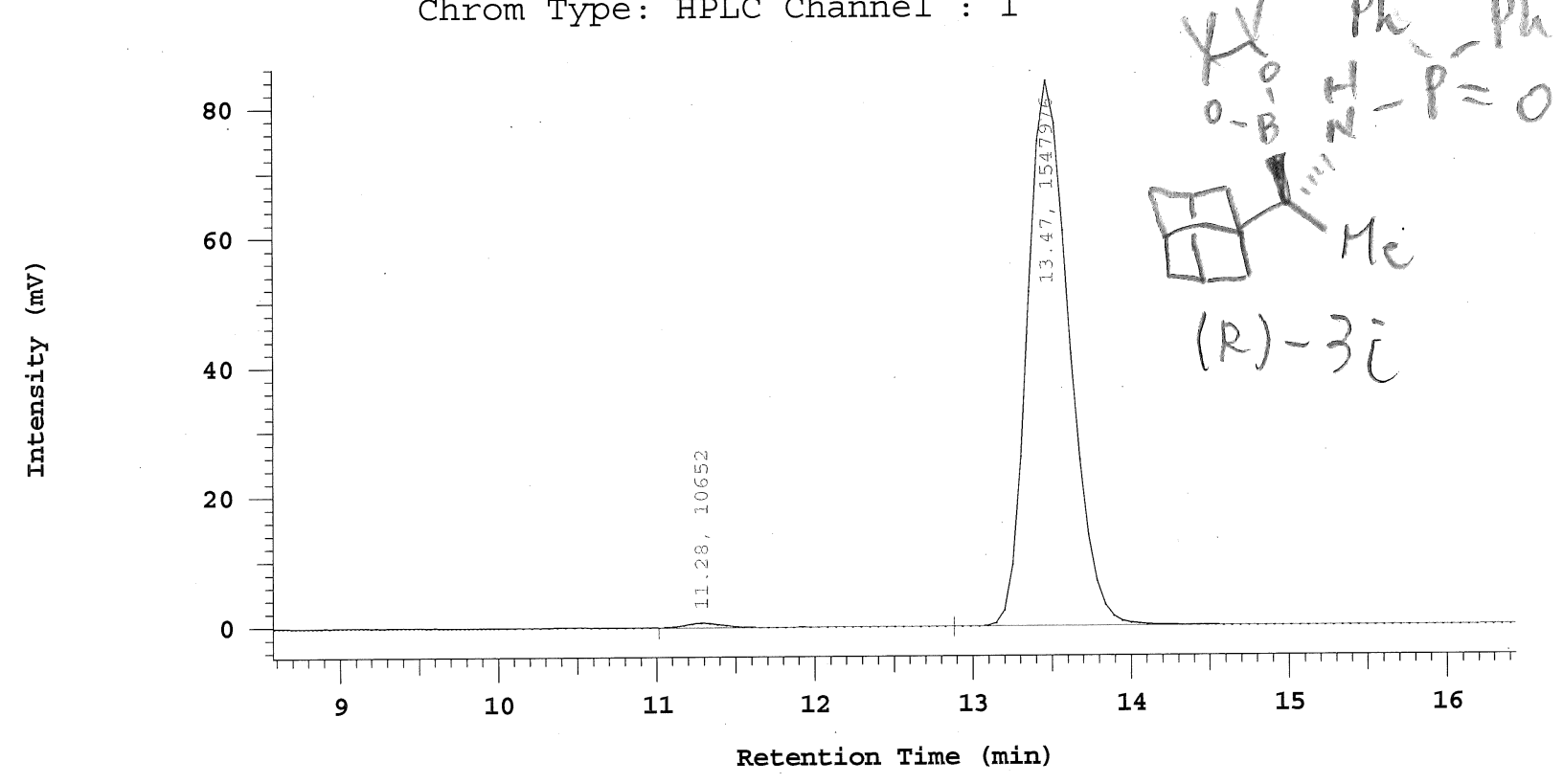

Processing Method: $10 / 90 \mathrm{iPrOH} / \mathrm{Hexane}$

Column Type: $\mathrm{OD}-\mathrm{H} 2$

Method Developer: Administrator

Pump A: L-2130

Pump A Solvent A: Hexane

Pump A Solvent B: 10/90 iProH/Hexane

Pump A Solvent C: iPrOH

Pump A solvent D: iPrOH

Method Description:

Chrom Type: HPLC Channel : 1

Peak Quantitation: AREA

Calculation Method: AREA\%

\begin{tabular}{rrrr} 
No. & RT & Area & Area \% \\
\hline 1 & 11.28 & 10652 & 0.683 \\
2 & 13.47 & 1547976 & 99.317 \\
\hline & & 1558628 & 100.000
\end{tabular}

Peak rejection level: 0 


\section{D-2000 Elite HPLC System Manager Report}

Analyzed Date and Time: 2020/06/29

$$
17: 26
$$

Reported Date and Time: 2020/06/29

$18: 55$

Processed Date and Time: 2020/06/29

$$
18: 55
$$

Data Path: C: \WIN32APP \D2000HSM Isocratic \DATA \3026

Processing Method: 10/90 iPrOH/Hexane

System (acquisition): Sys 1

Application(data): Isocratic HPLC

Sample Name: MUR-255-rac-OZ3-10\%

Injection from this vial: 1 of 1

Sample Description:
Series: 3026

Vial Number: 142

Vial Type: UNK

Volume: $10.0 \mathrm{ul}$

Chrom Type: HPLC Channel : 1
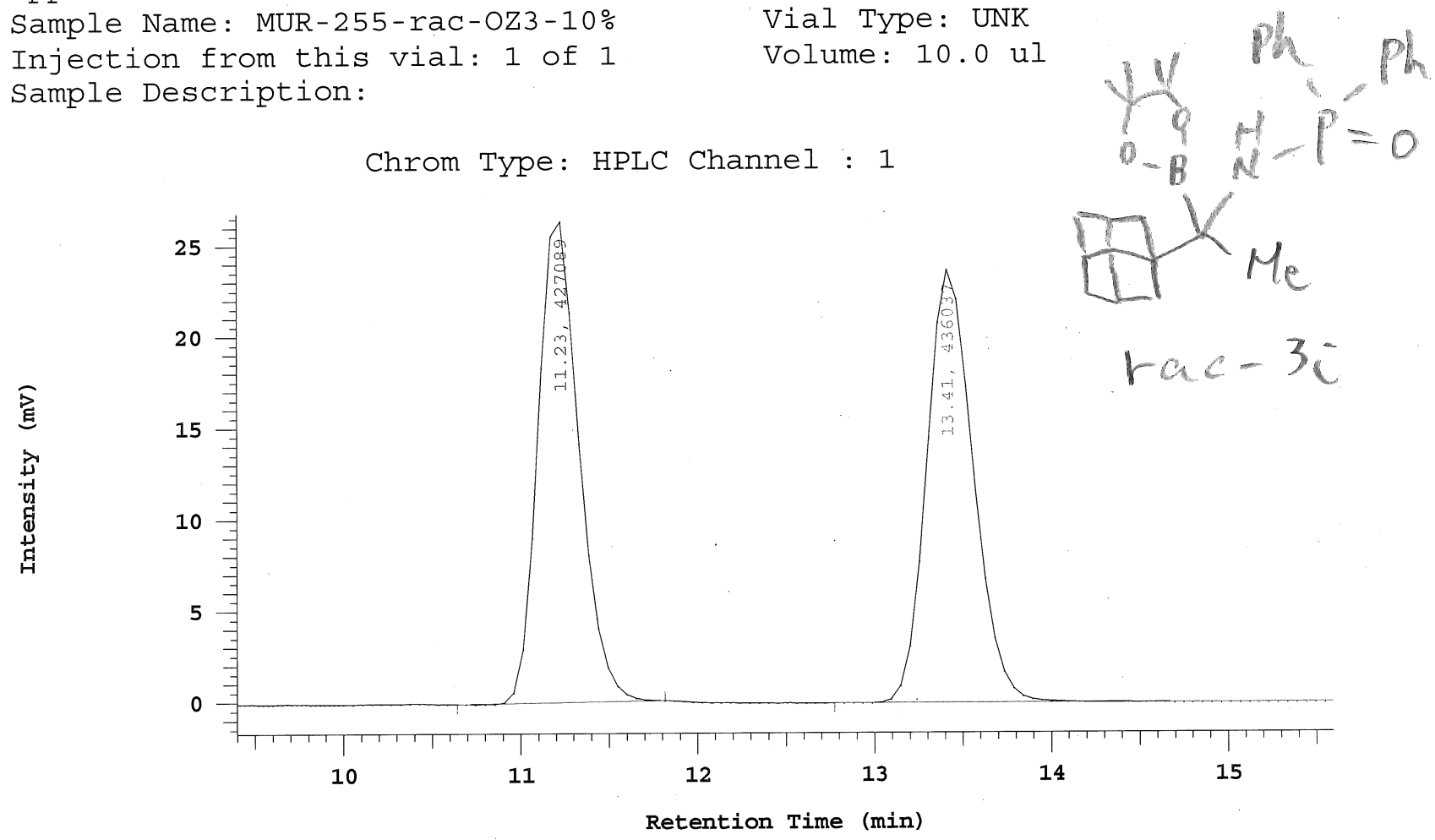

Processing Method: 10/90 iProH/Hexane

Column Type: OD-H 2

Method Developer: Administrator

Pump A: L-2130

Pump A Solvent A: Hexane

Pump A solvent C: iProH

Pump A Solvent B: 10/90 iProH/Hexane Pump A Solvent D: iPrOH

Method Description:

Chrom Type: HPLC Channel : 1

Peak Quantitation: AREA

Calculation Method: AREA\%

\begin{tabular}{ccrr} 
No. & RT & Area & Area \\
\hline 1 & 11.23 & 427089 & 49.482 \\
2 & 13.41 & 436037 & 50.518 \\
\hline & & 863126 & 100.000
\end{tabular}

Peak rejection level: 0 


\section{D-2000 Elite HPLC System Manager Report}

Analyzed Date and Time: 2020/06/13

Reported Date and Time: 2020/06/13

$$
13: 10
$$

Processed Date and Time: 2020/06/13

$$
14: 33
$$

Data Path: C: $\backslash$ WIN32APP $\backslash$ D2000HSM $\backslash$ Isocratic $\backslash$ DATA $\backslash 3003 \backslash$ Processing Method: 10/90 iPrOH/Hexane

System (acquisition): Sys 1

Application(data): Isocratic HPLC

Sample Name: MUR-232-OZ3-10\%

Injection from this vial: 1 of 1

Sample Description:
Series: 3003

Vial Number: 141

Vial Type: UNK

Volume: $10.0 \mathrm{ul}$

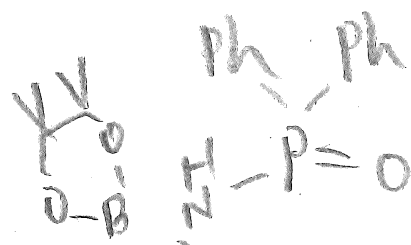

Chrom Type: HPLC Channel : 1
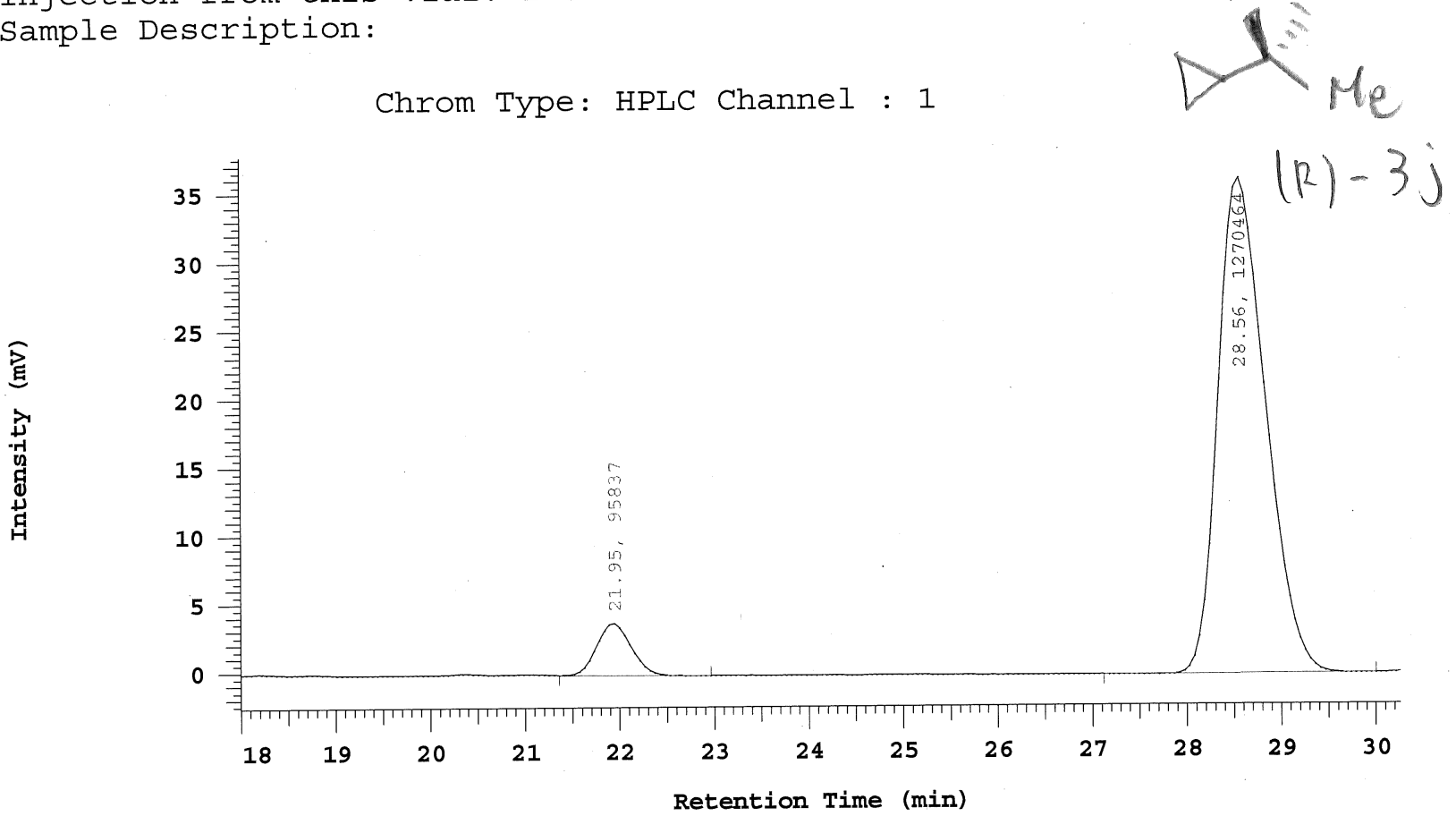

Processing Method: 10/90 iProH/Hexane

Column Type: OD-H 2

Method Developer: Administrator

Pump A: L-2130

Pump A Solvent A: Hexane

Pump A Solvent B: 10/90 iProH/Hexane

Pump A Solvent C: iPrOH

Pump A Solvent D: iPrOH

Method Description:

Chrom Type: HPLC Channel : 1

Peak Quantitation: AREA

Calculation Method: AREA\%

\begin{tabular}{rrrr} 
No. & RT & Area & Area \% \\
\hline 1 & 21.95 & 95837 & 7.014 \\
2 & 28.56 & 1270464 & 92.986 \\
\hline & & 1366301 & 100.000
\end{tabular}

Peak rejection level: 0 


\section{D-2000 Elite HPLC System Manager Report}

Analyzed Date and Time: 2020/06/13

Reported Date and Time: 2020/06/13

$$
14: 32
$$

Processed Date and Time: 2020/06/13

$$
16: 51
$$

Data Path: C: $\backslash$ WIN32APP $\backslash$ D2000HSM $\backslash$ Isocratic $\backslash$ DATA $\backslash 3004 \backslash$

Processing Method: 10/90 iPrOH/Hexane

System (acquisition): Sys 1

Application(data): Isocratic HPLC

Sample Name: MUR-231-rac-OZ3-10\%

Injection from this vial: 1 of 1

Sample Description:
Series: 3004

Vial Number: 142

Vial Type: UNK

Volume: $10.0 \mathrm{ul}$

Chrom Type: HPLC Channel : 1

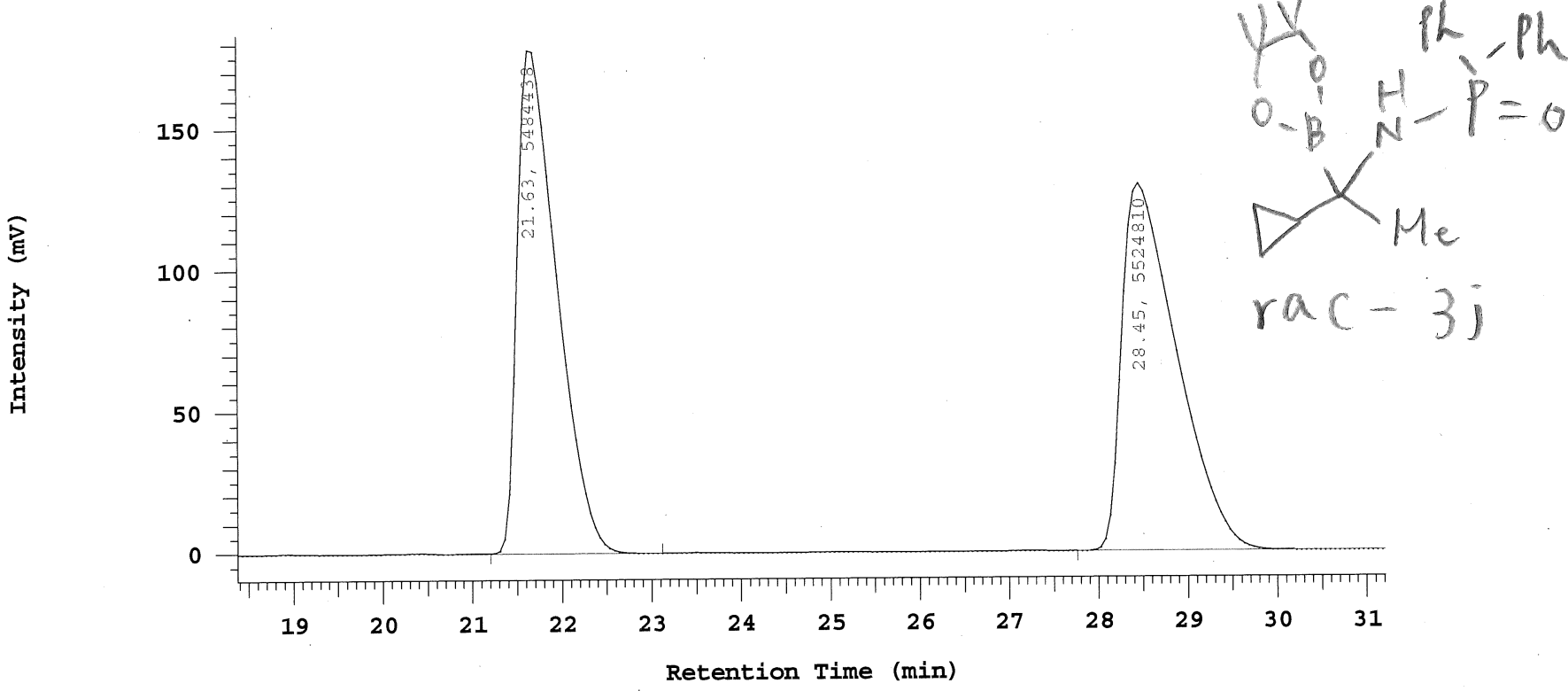

Processing Method: 10/90 iPrOH/Hexane

Column Type: OD-H 2

Method Developer: Administrator

Pump A: L-2130

Pump A Solvent A: Hexane

Pump A Solvent B: 10/90 iProH/Hexane

Pump A Solvent C: iProH

Pump A Solvent D: iPrOH

Method Description:

Chrom Type: HPLC Channel : 1

Peak Quantitation: AREA

Calculation Method: AREA\%

\begin{tabular}{rrrr} 
No. & RT & Area & Area \% \\
\hline 1 & 21.63 & 5484438 & 49.817 \\
2 & 28.45 & 5524810 & 50.183 \\
\hline & & 11009248 & 100.000
\end{tabular}

Peak rejection level: 0 


\section{D-2000 Elite HPLC System Manager Report}

Analyzed Date and Time: 2020/07/02

$$
16: 04
$$

Reported Date and Time: 2020/07/02

Processed Date and Time: 2020/07/02

$$
16: 40
$$

Data Path: C: \WIN32APP \D2000HSM Isocratic \DATA \3027\

Processing Method: 10/90 iPrOH/Hexane

System (acquisition): Sys 1

Series: 3027

Application(data): Isocratic HPLC

Sample Name: MUR-264-OZ3-10\%

Vial Number: 141

Vial Type: UNK

Injection from this vial: 1 of 1

Sample Description:

Volume: $10.0 \mathrm{ul}$

Chrom Type: HPLC Channel : 1

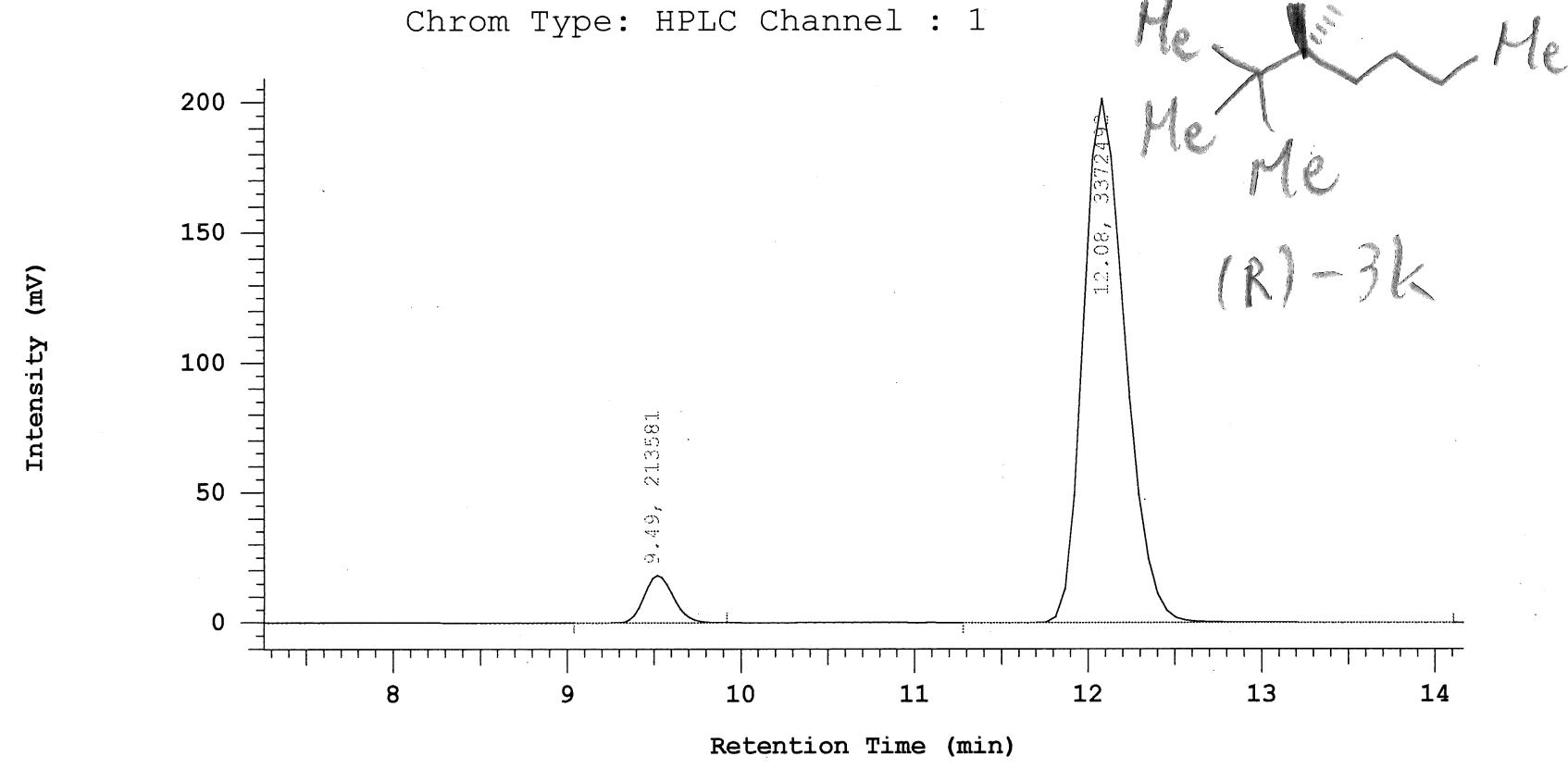

Processing Method: 10/90 iProH/Hexane

Column Type: OD-H 2

Method Developer: Administrator

Pump A: L-2130

Pump A Solvent A: Hexane

Pump A Solvent B: $10 / 90$ iProH/Hexane

Pump A Solvent C: iPrOH

Pump A Solvent D: iPrOH

Method Description:

Chrom Type: HPLC Channel : 1

Peak Quantitation: AREA

Calculation Method: AREA응

\begin{tabular}{rrrr} 
No. & RT & Area & Area $\%$ \\
\hline 1 & 9.49 & 213581 & 5.956 \\
2 & 12.08 & 3372492 & 94.044 \\
\hline & & 3586073 & 100.000
\end{tabular}

Peak rejection level: 0 


\section{D-2000 Elite HPLC System Manager Report}

Analyzed Date and Time: 2020/07/02 $16: 46$

Reported Date and Time: 2020/07/02 $17: 28$

Processed Date and Time: 2020/07/02

$$
17: 28
$$

Data Path: C: \WIN32APP \D2000HSM Isocratic \DATA \3028\

Processing Method: 10/90 iPrOH/Hexane

System (acquisition): Sys 1

Application(data): Isocratic HPLC

Sample Name: MUR-263-rac-OZ3-10\%

Injection from this vial: 1 of 1

Sample Description:

Series: 3028

Vial Number: 142

Vial Type: UNK

Volume: $10.0 \mathrm{ul}$

Chrom Type: HPLC Channel : 1

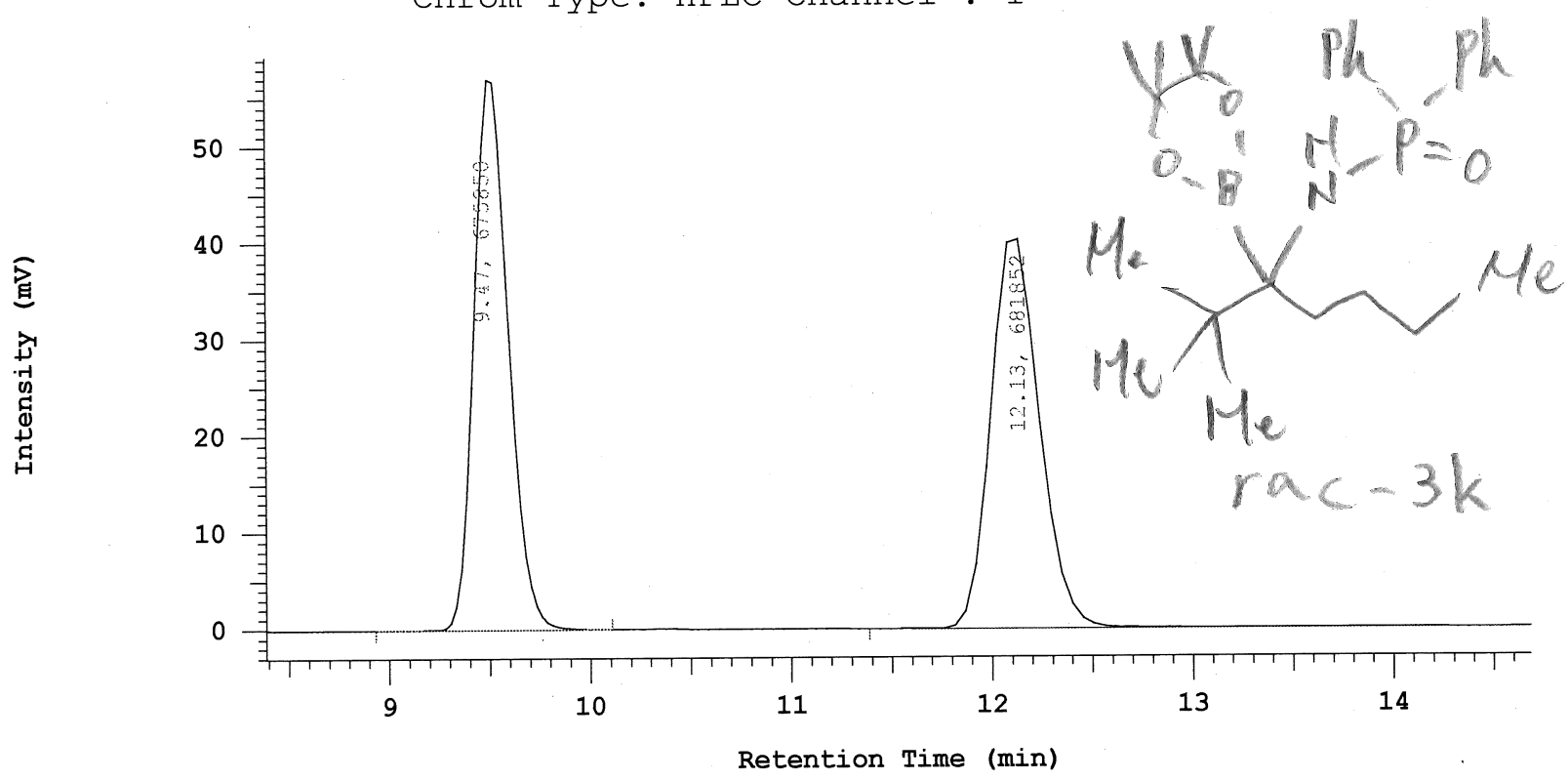

Processing Method: 10/90 iPrOH/Hexane

Column Type: OD-H 2

Method Developer: Administrator

Pump A: L-2130

Pump A Solvent A: Hexane

Pump A Solvent C: iPrOH

Pump A Solvent B: 10/90 iProH/Hexane

Pump A Solvent D: iPrOH

Method Description:

Chrom Type: HPLC Channel : 1

Peak Quantitation: AREA

Calculation Method: AREA응

\begin{tabular}{rrrr} 
No. & \multicolumn{1}{c}{ RT } & Area & Area \\
\hline 1 & 9.47 & 675850 & 49.779 \\
2 & 12.13 & 681852 & 50.221 \\
\hline & & 1357702 & 100.000
\end{tabular}

Peak rejection level: 0 


\section{D-2000 Elite HPLC System Manager Report}

Analyzed Date and Time: 2020/09/25

Reported Date and Time: 2020/09/25

Processed Date and Time: 2020/09/25

$11: 05$

Data Path: C: \WIN32APP \D2000HSM \Isocratic \DATA \3079

Processing Method: 10/90 iPrOH/Hexane

System (acquisition): Sys 1

Series: 3079

Application(data): Isocratic HPLC

Vial Number: 145

Sample Name: MUR-313-L22-OZ3-10\%

Injection from this vial: 1 of 1

Vial Type: UNK

Sample Description:

Volume: $10.0 \mathrm{ul}$

Chrom Type: HPLC Channel : 1

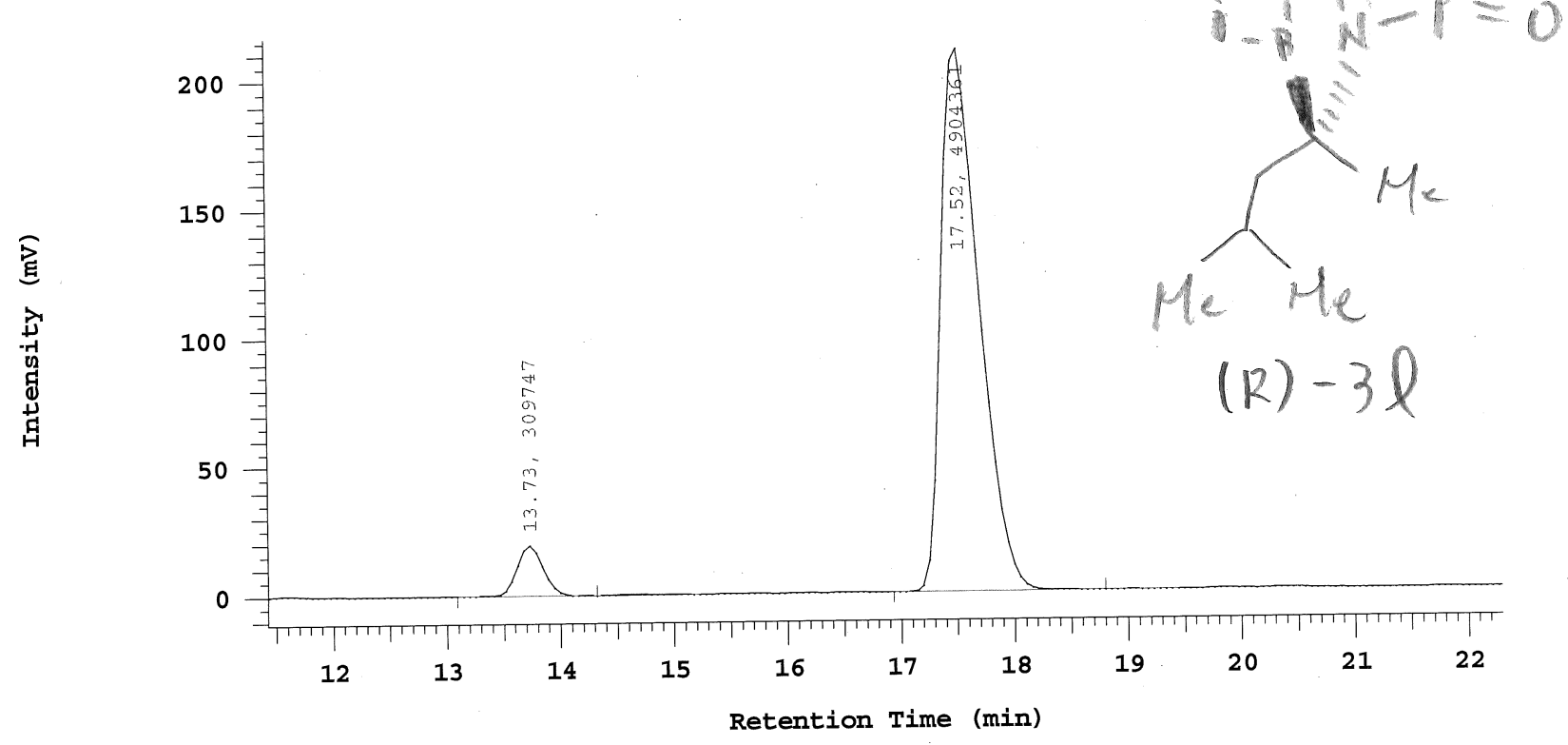

Processing Method: 10/90 iPrOH/Hexane

Column Type: OD-H 2

Method Developer: Administrator

Pump A: L-2130
Pump A Solvent A: Hexane
Pump A Solvent B: 10/90 iProH/Hexane
Pump A Solvent C: iPrOH
Pump A Solvent D: iPrOH

Method Description:

Chrom Type: HPLC Channel : 1

Peak Quantitation: AREA

Calculation Method: AREA\%

\begin{tabular}{rrrr} 
No. & RT & Area & Area $\%$ \\
\cline { 1 - 3 } & 13.73 & 309747 & 5.941 \\
2 & 17.52 & 4904361 & 94.059 \\
\cline { 2 - 4 } & & 5214108 & 100.000
\end{tabular}

Peak rejection level: 0 


\section{D-2000 Elite HPLC System Manager Report}

Analyzed Date and Time: 2020/09/25

$$
17: 34
$$

Reported Date and Time: 2020/09/25

Processed Date and Time: 2020/09/25

$$
19: 57
$$

Data Path: C: \WIN32APP \D2000HSM \Isocratic \DATA \3081

Processing Method: 03/97 iPrOH/Hexane

System (acquisition) : Sys 1

Series: 3081

Application(data): Isocratic HPLC

Vial Number: 146

Sample Name: MUR-314-rac-OZ3-10\%

Vial Type: UNK

Injection from this vial: 1 of 1

Volume: $10.0 \mathrm{ul}$

Sample Description:

Chrom Type: HPLC Channel : 1

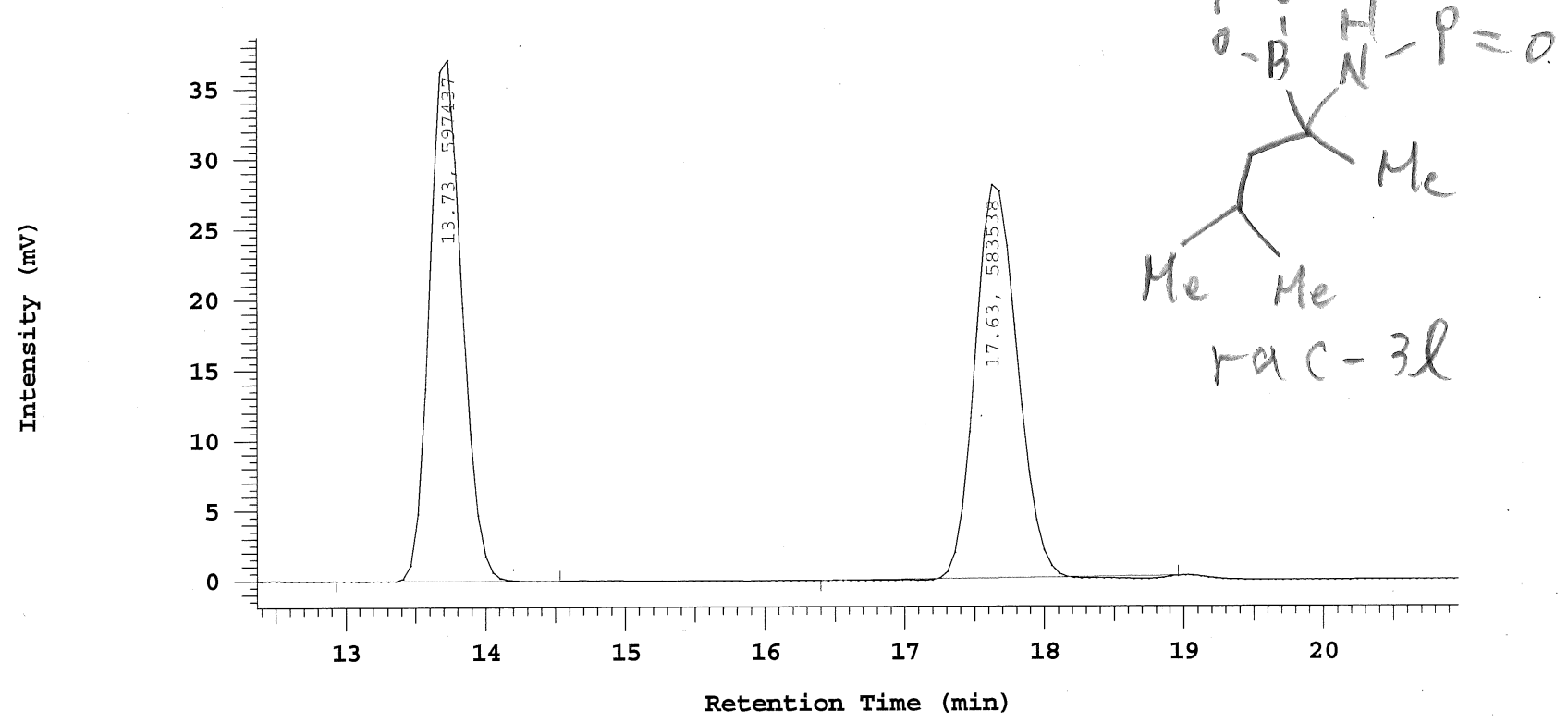

Processing Method: $03 / 97 \mathrm{iPrOH} / \mathrm{Hexane}$

Column Type: OD-H 2

Method Developer: Administrator

Pump A: L-2130

Pump A Solvent A: Hexane

Pump A Solvent B: 10/90 iPrOH/Hexane

Pump A Solvent C: iProH

Pump A solvent D: iPrOH

Method Description:

Chrom Type: HPLC Channel : 1

Peak Quantitation: AREA

Calculation Method: AREA\%

\begin{tabular}{rrrr} 
No. & RT & Area & Area \% \\
\hline 1 & 13.73 & 597437 & 50.588 \\
2 & 17.63 & 583538 & 49.412 \\
\hline & & 1180975 & 100.000
\end{tabular}

Peak rejection level: 0 


\title{
D-2000 Elite HPLC System Manager Report
}

Analyzed Date and Time: 2020/10/16

$$
21: 24
$$

Reported Date and Time: 2020/10/16

Processed Date and Time: 2020/10/16

$$
23: 15
$$

Data Path: C: \WIN32APP \D2000HSM \Isocratic \DATA \3097\

Processing Method: 05/95 iPrOH/Hexane

System (acquisition): Sys 1

Application(data): Isocratic HPLC

Sample Name: MUR-326-2-OZ3-5\%

Injection from this vial: 1 of 1

Sample Description:

\author{
Series: 3097 \\ Vial Number: 143 \\ Vial Type: UNK \\ Volume: $10.0 \mathrm{ul}$
}

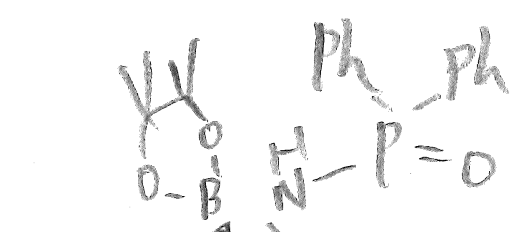

Chrom Type: HPLC Channel : 1

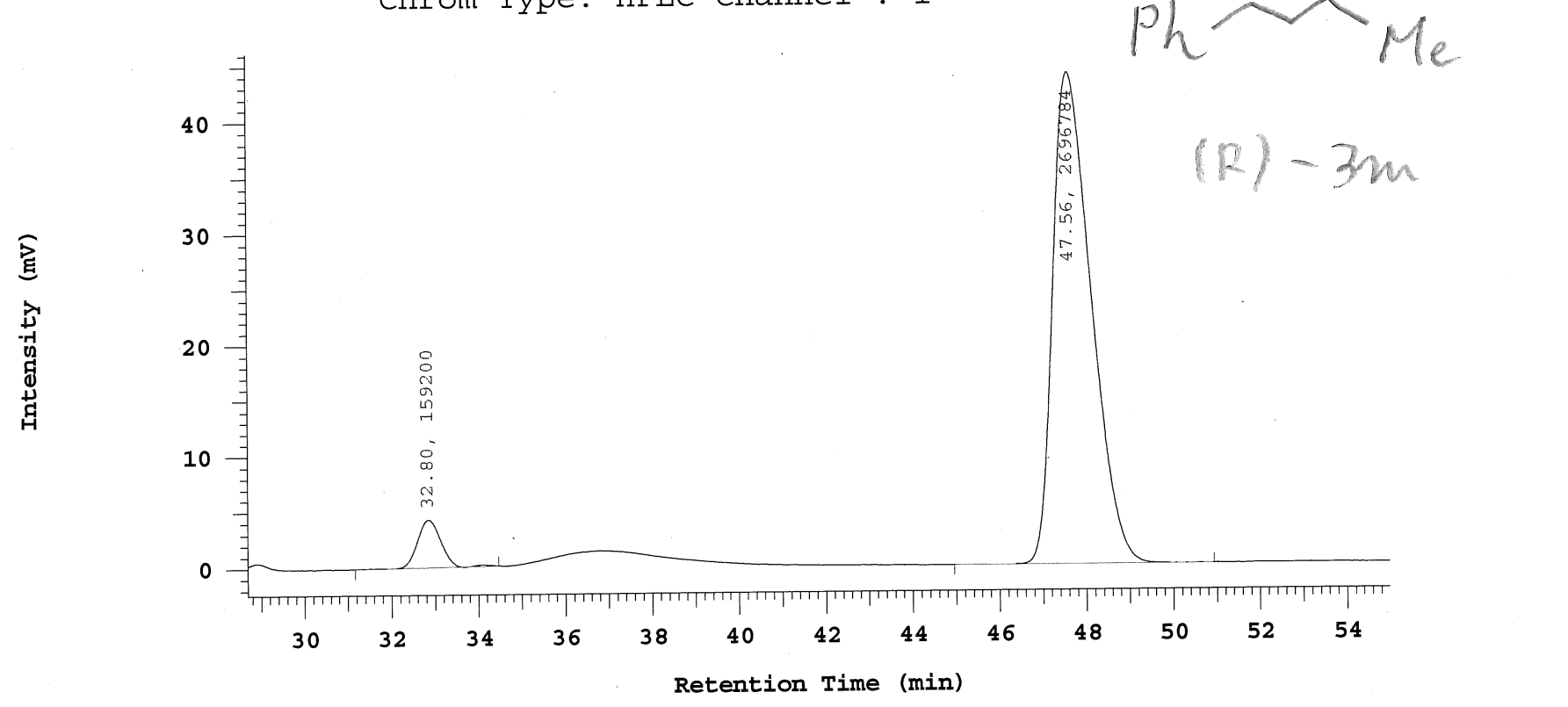

Processing Method: 05/95 iPrOH/Hexane

Column Type: OD-H 2

Method Developer: Administrator

Pump A: L-2130

Pump A Solvent A: Hexane

Pump A Solvent B: 10/90 iPrOH/Hexane

Pump A Solvent C: iPrOH

Pump A Solvent D: iPrOH

Method Description:

Chrom Type: HPLC Channel : 1

Peak Quantitation: AREA

Calculation Method: AREA\%

\begin{tabular}{rrrr} 
No. & RT & Area & Area \% \\
\hline 1 & 32.80 & 159200 & 5.574 \\
2 & 47.56 & 2696784 & 94.426 \\
\hline & & 2855984 & 100.000
\end{tabular}

Peak rejection level: 0 


\section{D-2000 Elite HPLC System Manager Report}

Analyzed Date and Time: 2020/10/16

Reported Date and Time: $\begin{aligned} & 2020 / 10 / 17 \\ & 00: 18\end{aligned}$

$$
22: 56
$$

Processed Date and Time: 2020/10/17

$00: 18$

Data Path: C: \WIN32APP \D2000HSM \Isocratic \DATA \3098\

Processing Method: 05/95 iPrOH/Hexane

System (acquisition): Sys 1

Application(data): Isocratic HPLC

Sample Name: MUR-327-OZ3-10\%

Injection from this vial: 1 of 1

Sample Description:

Series: 3098

Vial Number: 141

Vial Type: UNK

Volume: $10.0 \mathrm{ul}$

Chrom Type: HPLC Channel : 1

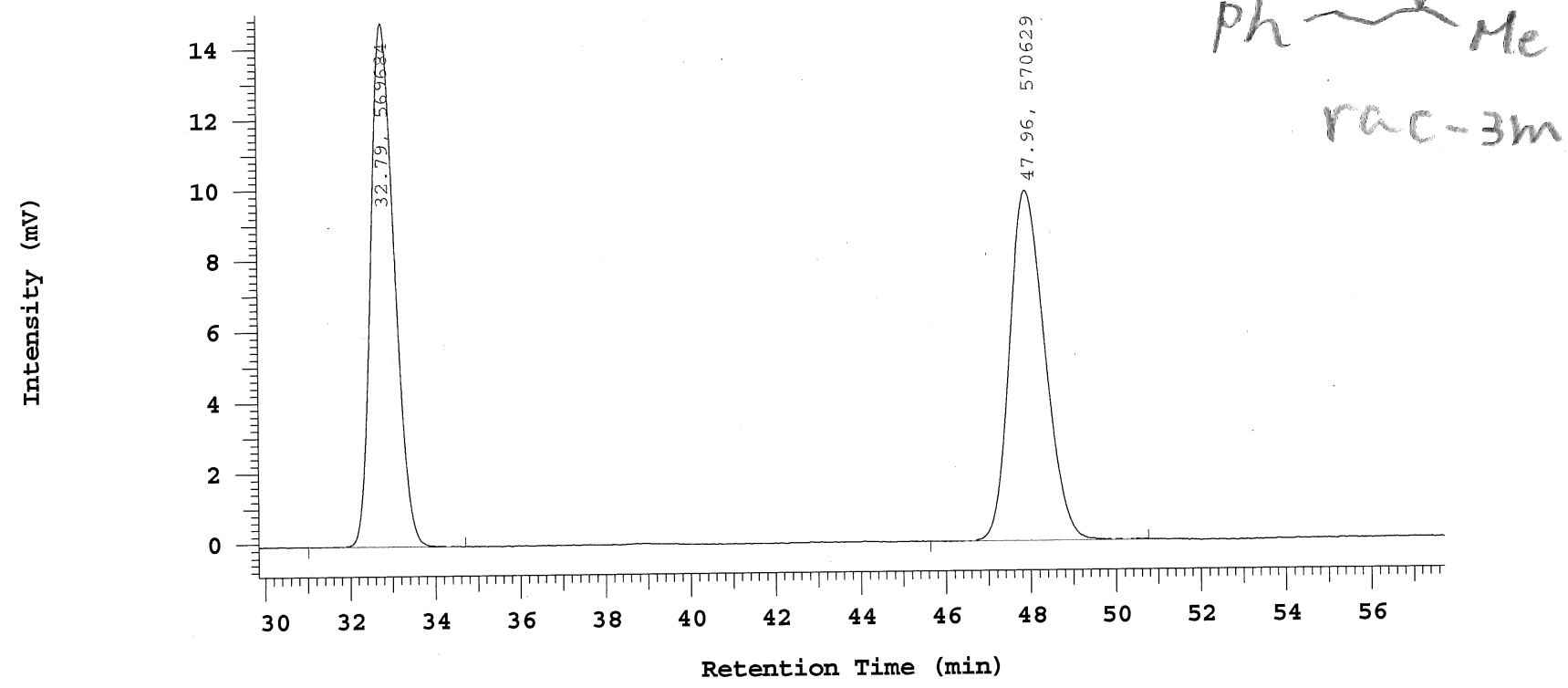

Processing Method: 05/95 iPrOH/Hexane

Column Type: OD-H 2

Method Developer: Administrator

Pump A: L-2130

Pump A Solvent A: Hexane

Pump A Solvent B: 10/90 iProH/Hexane

Pump A Solvent C: iPrOH

Pump A Solvent D: iPrOH

Method Description:

Chrom Type: HPLC Channel : 1

Peak Quantitation: AREA

Calculation Method: AREA\%

\begin{tabular}{lrrr} 
No. & RT & Area & Area \% \\
\cline { 1 - 3 } \cline { 3 - 4 } 2 & 32.79 & 569684 & 49.959 \\
\cline { 3 - 4 } & 47.96 & 570629 & 50.041 \\
\hline & & 1140313 & 100.000
\end{tabular}

Peak rejection level: 0 


\section{D-2000 Elite HPLC System Manager Report}

Analyzed Date and Time: 2020/10/31

Reported Date and Time: 2020/10/31

$18: 04$

Processed Date and Time: 2020/10/31

$$
19: 33
$$

Data Path: C: \WIN32APP \D2000HSM Isocratic \DATA \3102\

Processing Method: 10/90 iPrOH/Hexane

System (acquisition): Sys 1

Application(data): Isocratic HPLC

Sample Name: MUR-333-rac-OZ3-10\%

Injection from this vial: 1 of 1

Sample Description:
Series: 3102

Vial Number: 148

Vial Type: UNK

Volume: $10.0 \mathrm{ul}$

Chrom Type: HPLC Channel : 1

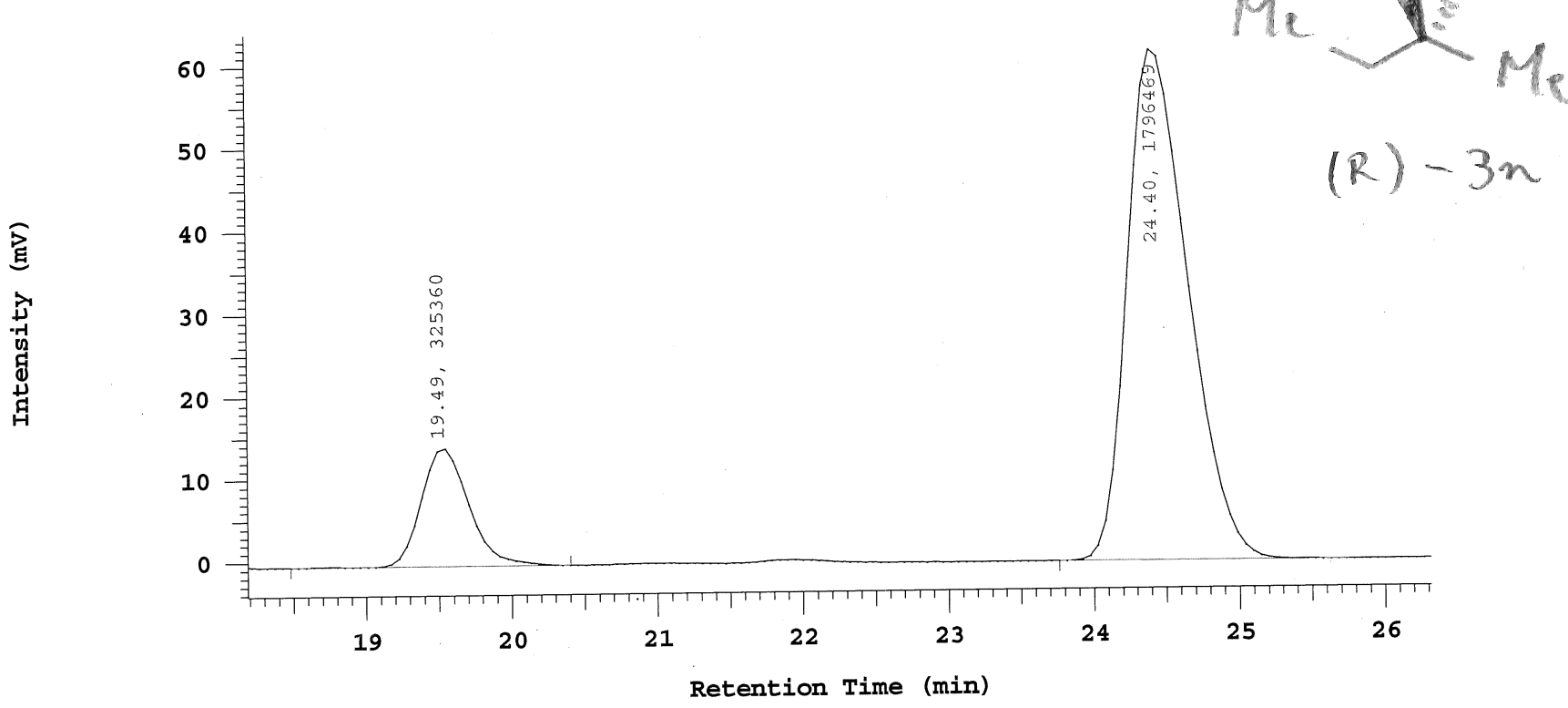

Processing Method: 10/90 iPrOH/Hexane

Column Type: OD-H 2

Method Developer: Administrator

Pump A: L-2130

Pump A Solvent A: Hexane

Pump A Solvent B: 10/90 iPrOH/Hexane

Pump A Solvent C: iPrOH

Pump A solvent D: iPrOH

Method Description:

Chrom Type: HPLC Channel : 1

Peak Quantitation: AREA

Calculation Method: AREA\%

\begin{tabular}{lrrr} 
No. & RT & Area & Area $\%$ \\
\cline { 1 - 3 } \cline { 3 - 4 } 2 & 19.49 & 325360 & 15.334 \\
\cline { 3 - 4 } & 24.40 & 1796469 & 84.666 \\
\hline & & 2121829 & 100.000
\end{tabular}

Peak rejection level: 0 


\section{D-2000 Elite HPLC System Manager Report}

Analyzed Date and Time: 2020/10/31

Reported Date and Time: 2020/10/31

$15: 25$

$16: 49$

Processed Date and Time: 2020/10/31

$16: 48$

Data Path: C: $\backslash$ WIN32APP $\backslash$ D2000HSM $\backslash$ Isocratic $\backslash D A T A \backslash 3101 \backslash$

Processing Method: 10/90 iPrOH/Hexane

system (acquisition): Sys 1

Application(data): Isocratic HPLC

Sample Name: MUR-332-rac-OZ3-10\%

Injection from this vial: 1 of 1

Sample Description:
Series: 3101

Vial Number: 148

Vial Type: UNK

Volume: $10.0 \mathrm{ul}$

Chrom Type: HPLC Channel : 1

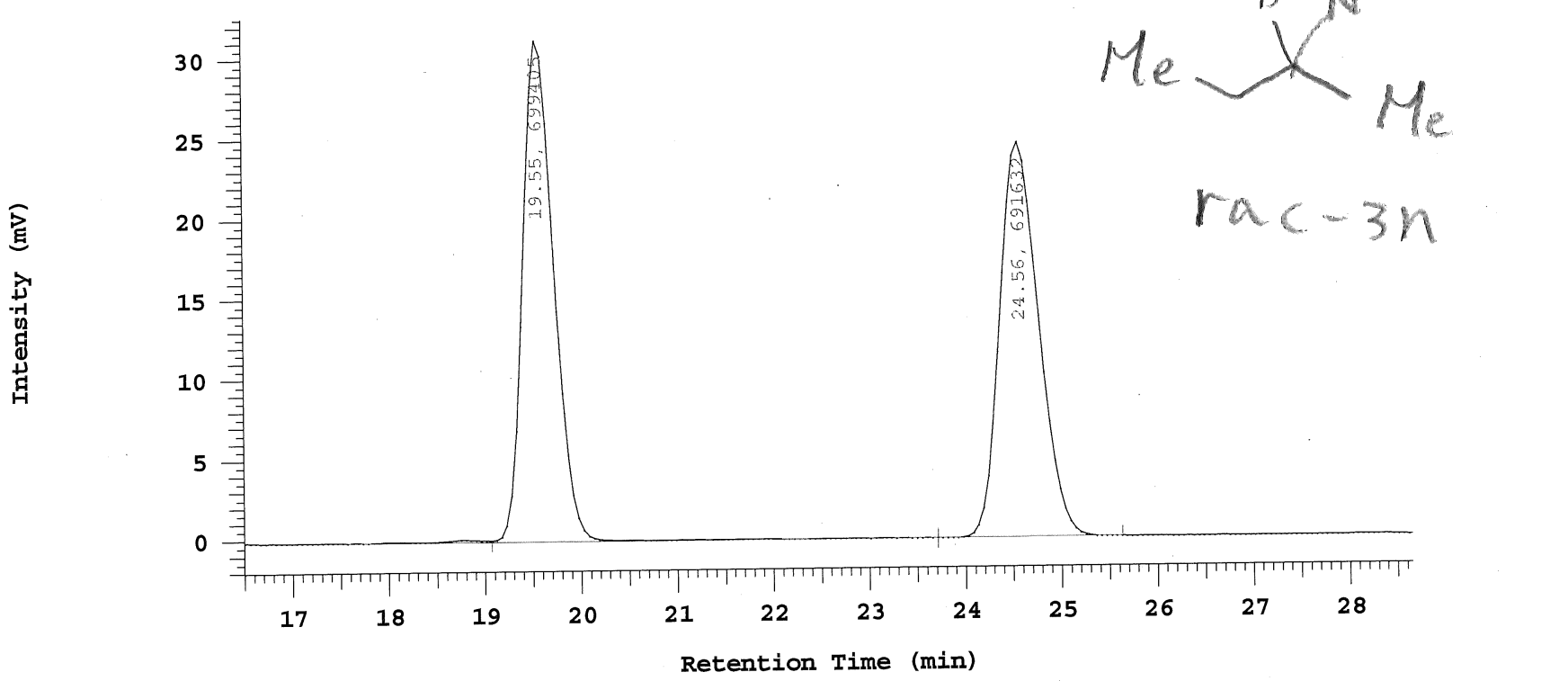

Processing Method: 10/90 iPrOH/Hexane

Column Type: OD-H 2

Method Developer: Administrator

Pump A: L-2130

Pump A Solvent A: Hexane

Pump A Solvent B: 10/90 iPrOH/Hexane

Pump A Solvent C: iPrOH

Pump A Solvent D: iPrOH

Method Description:

Chrom Type: HPLC Channel : 1

Peak Quantitation: AREA

Calculation Method: AREA\%

\begin{tabular}{rrrr} 
No. & RT & Area & Area \% \\
\hline 1 & 19.55 & 699405 & 50.279 \\
2 & 24.56 & 691632 & 49.721 \\
\hline & & 1391037 & 100.000
\end{tabular}

Peak rejection level: 0 